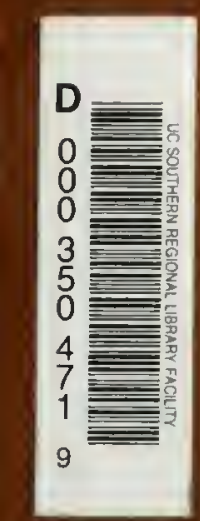

fornia
nal
$y$ 
UNIVERSITY OF CALIFORNIA

AT LOS ANGELES

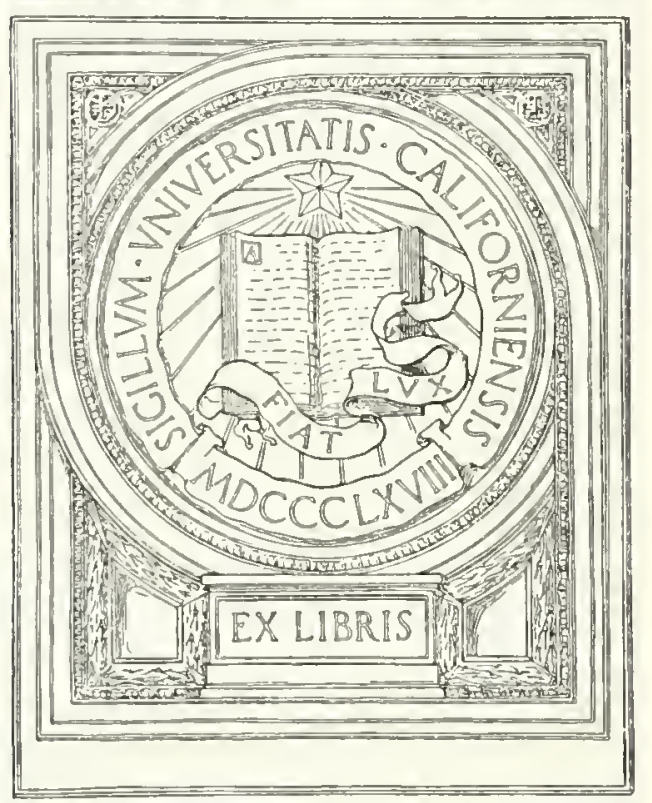

ROBERT ERNEST COWAN 




\section{Smithsonian Uontributions to linowledge.}

\section{T I D A L 0 B S E R V A T I 0 N S}

IX THE

\section{A R C T I C S E A S.}

BY

ELISIIA KENT KANE, II.D., U.S.N.

MADE DURING THE SECOND GRINAELL EXPEDITION IN SEARCII OF SIR JOIN FRANKLIN, IN 1853, 1554, AND 1855, AT VAN RENSSELAER IARBOR.

REDUCED AND DISCUSSED,

BY

C H A R L E S A. S C H O T T, ASSISTANT U. S. COAST SURVEX.

W A S H IN G TON C I T Y:

PUBLISHED BY THE SIITHSONIAN INSTITUTION. OCTOBER, 1860. 


SMITHSONIAN CONTRIBUTIONS TO KNOWLEIDGR.

\section{T' I D A L O B S E R V A T I 0 N S}

\section{A R C T I C S E A S.}

BY

BLISHA KENT KANE, M. I., I.S.S.

MADE DURING TIIE SECOND GRINXELL LXPEDITION IN SEARCII OF SIR JOIN FRANKLIN, IN 1853, 1854, AND 1855, AT YAN RENSSELAER IIARDOR.

REDTCEH ANI DISCUSSET,

IB $\mathrm{Y}$

C H A R I ES A. S C H O T T,

asistant T. S. COAST ATRTEY. 



\section{CONTENTS.}

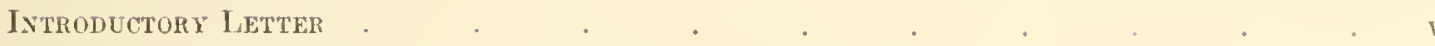

Explanatory and introductory remarks . . . . . . . . . . . . 1

Record of tidal observations at Van Renssclacr Harbor, 1853-t-5 . . . . . 5

Diseussion of half-monthly inequality in time and height . . . . . . . . 67

Effect of changes of the moon's declination and parallax . . . . . . . 72

es Discussion of the dimmal incquality in height and time . . . . . . . Tt

क Investigation of the form of the tidal wave . . . . . . . . . . is

Note on the effect of wind on the tides . . . . . . . . . . . . 1

Note on the progress of the tidal ware and depth of the sea . . . . . . . . . .

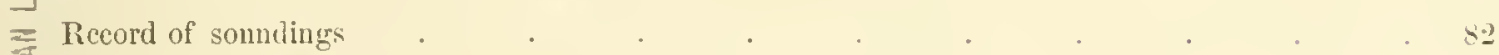

Appendx-containing a tidal record at Wolstenholm Sound, Commander Saunders, 1849-50, with forr plates 



\section{INTRODUOTORY LET'TER。}

l'rofessor JosepH Henry, LL. 1.,

Wasingtos, July 4th, 1860.

Secretary of the Smithsonien Institution:

DEAR SIR: The records of the tidal observations made under the direction of Dr. Kane, in the second Grimell Expedition to the Aretic Regions, were placed in my hands by lis late lanented father, Julge Kane, in December, $185 \%$.

Dr. Kane had selected Assistant Charles A. Schott, of the U. S. Coast Survey, for the reduction of a considerable portion of the obscrvations made on that expedition; and I, therefore, placed them in Mrr. Schott's posscssion for reduction, and recommend his paper for publication in the "Smitlsonian Contributions to Knowledge." It is proper to state that the computations were at the expense of the Smitlsonian Institution. 'This is the sixth and last paper of the series.

Very respectfully; yours,

A. I) B.LCIIE,

Superintendent. L. S. Cuaś Surry. 



\section{RECORD AND REDUCTION OF TIIE TIDES.}

The observations and discussion of the ticles at Van Rensselacr IInrbor, the winter quarters of the Advance during 1553-54 and 18.54-55, will form the last of the series of papers on the results of the expedition, prepared by me for publication.

Occasional tidal observations were made after passing Smith Straits, when, owing to the peculiar navigation through the narrow openings betwcen the coast and the bay ice, the ressel was much exposed to the tidal action, frequently grounding at low water, and otherwise, by taking advantage of high ticles, slowly adrancing to her winter quarters.

The bay, near the head of which the Adrance was laid up, and used as the winter quarters by Dr. Kane's party, is freely exposed to the north (true) and northwest; the indentation of the shore line is about fire miles; some rocky islands are situated within the bay.

Shortly after the ressel entered the harbor a tide staff was arranged, and a series of tidal observations was commenced on September 11, 1853, and continued, with occasional interruptions (partly owing to defects in the pulley-gauge, afterwards rigged up, and partly owing to other unavoidable accidents) till the 24 th of January, 1855, on which date the regular log book appears to have been discontinued.

The sereral series of observations during this period are of rery unequal value, as will appear in the detailed examination and discussion of the results. The difficulties to be orercome in the attempt to secure a reliable set of observations were considerable, those of a physical nature being the greatest. 'The observations with the staff or sounding line are subject to irregularities from a slow morement of the ressel, which, though imbedded in ice during the greater part of the year, is yet not stationary; these observations may also be affected by the softness of the bottom; the observations by means of a pulley tide gauge may be defective, on account of a slow drift of the ressel and motion of the ice ficld, also in consequence of a lengthening or shortening of the rope, or it may be in consequence of slipping of the rope on the circumference of the wheel. The latter defect, or one similar in its nature, has been a source of much annoyane, requiring the application of corrections to the readings, in order to refer all observations to the same zero of the scale. There is another defect to which pulley-ganges are subject, namely, the gradual rise of the ressel, in consequence of the consumption of prorisions and fuel. Notices of thesc defects will appear in the subsequent discussion.

The pulley-gauge is described by $\mathrm{D}$ r. Kane, in rolume $\mathrm{I}$ of the Narative, p. 117, as follows: "Our tide register was on board the ressel, a simple pulley-gauge, 
arranged with a wheel and index, and dependent on her rise and fall for its rotation."

In order to ascertain the nature of the tides, as well as the degree of accuracy of the different observations, the readings were roughly plotted for a first examination; the following series were found suitable for discussion:-

Series I. From October 10th, 1853, to December 2sth, 1853.-This series, with the exception of three days, is complete; the observations in the latter part of December appear to be of less reliable character. The observations between September 11 and October 4,1853 , are too fragmentary to be used. The pulley-gange observations between October 4 and October 9 scem to have been only experimental. The hourly readings are superseded by half-hourly readings on Niovember s, and continue half hourly, day and night, to the end of the series. After Norember 2s, corrective soundings were taken at noon each day. In order to make use of these soundings, the mean depth of the watcr at the anchorage was derluced from them as follows:-

\begin{tabular}{|c|c|c|c|}
\hline \multicolumn{4}{|c|}{ Mean reading. } \\
\hline Decenber, & 1,53 & 43.5 & 3I soundings (at moou). \\
\hline Ianuary, & $185 \mathrm{t}$ & 44.9 & 21 \\
\hline Feीpमाy, & "6 & 443 & 17 \\
\hline Mareh, & " & 43.3 & 19 \\
\hline I pril, & “ & 41.5 & 20 \\
\hline May, & "6 & 13.5 & 9 \\
\hline
\end{tabular}

The indivilual soundings will appear in the record following.

Nean deptly of water at anchornge, in winter, 1853-54, 43.6 feet, as obtained from 117 soundings. The monthly mean values for the tidal level accord well, and show that no lateral change took place in the position of the brig (or else that the bottom was level). It will be secm that for Scries I the reading 7.0 was arlopted to express the incan level, the zero of the scale was, therefore, at an clevition of 36.6 fert from the bottom. The readings of the pulley guage are expressed in feet, ${ }^{2}$ as 1 have been informed by Mr. Sonntag.

Srums I1. From Jamury ?8th, 1854, to A pril 7th, 1854.- 'T'he double lialf-hourly rearlings of the pulley-gange are continued. 'The series is complete with the exception of ten davs, which hatl to be omitted. The register broke January 22: observations commenced January '24th, but were not sufficiently regular for use

\footnotetext{
2 The following note is appended: Onc end of the cord represented a fixed point, by being anchored to the bottom; the frec end, with an attached weight, rose and fell with the lirig, and recorderl its motion on the grooved circumferenee of a wheel. "l'his methed was liable to oljections, but it was correctel by daily sommlings. 'Tlue movenents of anr vessel partuok of those of the floe in which she was inberled, and were unaceompanied by any lateral deviation.

a 'The following is an extrict from Mr. Somntag's letter to me, llated Yew York, Mareh 23, 1860 :

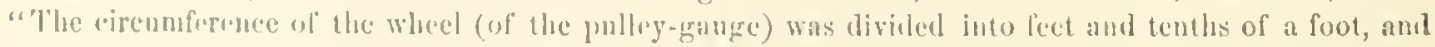

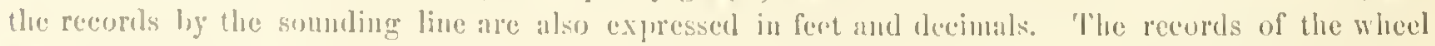
are very uncertuin, as olten the rope slid orer the whecl without turning it, owing to the ice which surrounded the axis."
} 
until Jannary 28 th. The corretive somulings at noon are continued, with occasional omissions, thromghout this series. After 1 pril 7 th there is a breat in the observations, those between the 14 th and 20 th appear to be irregular.

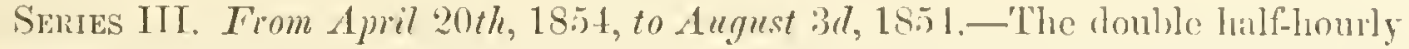
reatings of the pulley-gange contime to Mily 5th, after which date single latllourly rearlings are recorded. The corrective somolings rease on the 12th of May. Intermptions ocem between May the and May th, also on Ju! Sth, also between July 15th and 18 th, and between July 20 th and the 2Sth. On the Sth of Angut the brig was released from her ice cradle, and rose wo and a half feet; occasional warpings of the brigr after this date render the olserrations worthless. On the 23d of August the brig was in but seren feet of water, and grounded.

Series IV. From September Tth, 185t, to October 22d, 185t.-The hourly observations assume again a more regular appearance on the ith of September; they were taken with the sounding line, and are expressed in futhoms and feet (as stated in a note, August 12th). The following note is of October 21st, 18.5: "The tide register as yet not rigged, observations very faulty by sounding line." 'The irregularities increase after this date; on the 15 th of November following, the tide register was arranged, and observations (hourly) commenced on the 17 th; the slip. ping of the rope, however, was of so frequent occurrence and of so great an extent, that it was considered better to take no further notice of these observations; the record continues to January 24 th, 1855 , when the strength of the party no longer permitted due attention to the tidal phenomena.

It was apparent that before any closer insight into the nature of these tides conld be obtained, they must first be reduced to the same zero or mean level of the sea. To effect this in a manner apparently best suiting the case, and otherwise mobjectionable, two curved lines were traced on the diagrams, the upper one mveloping the highest high water of each day, the other enveloping the lowest low water of each day; in tracing these lines some allowance was made, when necessary, for disturbing causes, so as to obtain tolerably smooth curves; cases of abrupt changes were, of course, treated accordingly. A line, equichistant from these curres, was assumed as representing the mean level, and when straightened out was adopted as axis of the mean level of the sea. The corrections to refer each observation to this adopted mean level; or, in other words, the corrections required to refer each observation to the same zero of the scale, so as to make them comparable with each other, were taken from the projection, and are given in the column headed "reduction," in the following record.

This method of treatment excludes necessarily in Series I, IJ, and III, any discussion of the variation in the mean level of the sea, the oscillations of which have been found small at other places. As an illustration of this, the tictes at Singapore might be referred to; the Rev. W. Whewell (7th series of rescarches on the tides, P'hil. Trans. of the Roy. Soc., Part I, 1837), finds for these tides that, if a line is drawn representing the mean height (midway between high and low water each day) it is very nearly constant, thongh the successive low waters often differ ly six 
feet (on account of the diurnal inequality), the mean level only oscillates through a few inches. It appears from Mr. Lloyd's paper (Phil. Trans. of 1831) that the mean level at Sheerness is higher in spring tides than in neap tides by seren inches nearly; also there secms to be no doubt (as shown by Mr. Whewell, Phil. Trans., 1839 and 1840 ) that the mean level increases as the moon's dechination increases, amounting to three inches at Plymouth, when the moon's declination is $25^{\circ}$; at Petropaulofsk and Novo-Arlihangelsk the mean level rises as the moon's declination increases.

The use of the soundings intended to furnish corrections to the readings of the pulley-gauge is in many cases a doubtful remedy, on account of the continued change in the zero of the wheel's index; in fact, it would have required numerous soundings at other hours than noon. As it is, a combination of the corrections by enveloping curves and soundings had to be adopted. Thus, for December 5th, soundings at noon 43.0 feet (sce record further on), mean level 36.6 , hence reading of scale at noon 6.4; reading of pulley-gange at that hour 19.0, correction by curve -12.5 , corrected reading 6.5 , which agrees with the first number; this is, however, a very farorable case. For intermediate hours the correction as given by the curves serve as guides. The reduction to the same level affects the times generally very little.

The following table contains the soundings taken at noon between the interval of the first and second series, those taken during the series being given in the record.

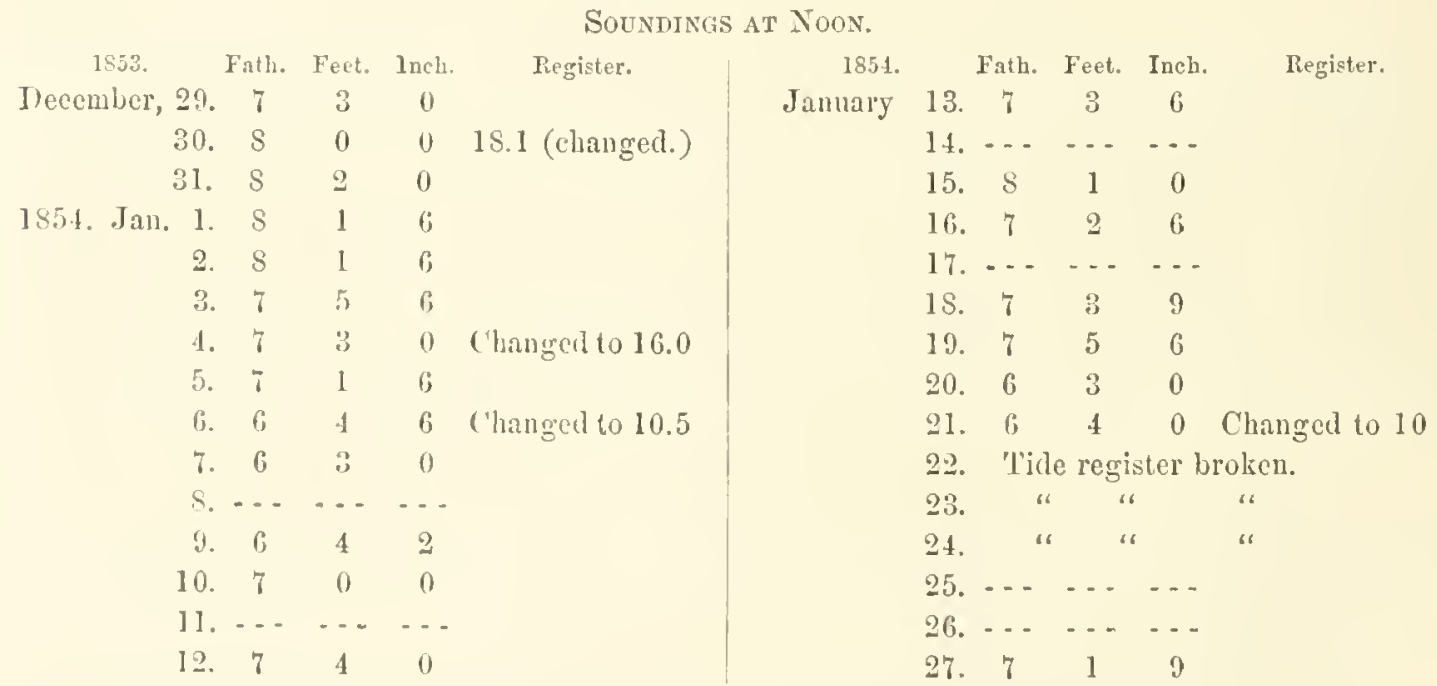

'The following sonndings were taken between the second and third series:-

\begin{tabular}{|c|c|c|c|c|c|c|c|c|}
\hline $\begin{array}{l}\text { la5. } \\
\text { April }\end{array}$ & 8. & $\begin{array}{c}\text { Fath. } \\
\text { i; }\end{array}$ & $\begin{array}{c}\text { leet. } \\
5\end{array}$ & $\begin{array}{c}\text { Inehes. } \\
\text { f }\end{array}$ & $\begin{array}{l}1854 . \\
1 \text { pril } 16 .\end{array}$ & $\begin{array}{c}\text { Fath. } \\
7\end{array}$ & $\begin{array}{c}\text { Feet. } \\
5\end{array}$ & $\begin{array}{c}\text { Inches. } \\
6\end{array}$ \\
\hline & 9. & 6 & 4 & 0 & \multicolumn{4}{|c|}{ (Fall 15 feet 8 inches.) } \\
\hline & 10 & 7 & 11 & 6; & (IT. & 6 & 5 & 0 \\
\hline & 11. & (i) & 5 & 6 & \multicolumn{4}{|c|}{ at 20 minutes to 5.$)$} \\
\hline & 13. & 7 & 1 & 11 & (18. & 6 & 0 & 0 \\
\hline & 11.1 & 7 & 5 & 6 & \multicolumn{4}{|c|}{ at $S^{\ln } 15^{\text {in }}$ I. M.) } \\
\hline & 15. & 8 & 11 & 1) & 19. & fi & 2 & 0 \\
\hline
\end{tabular}

(I,uw water to ligh water I 1 ft. 8 inch.)

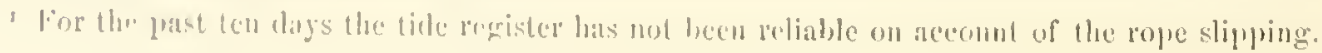


The note of February 3d, 1854, is very instructive in regard to the effect of the tides on the ice floe, viz: "The enomous elevation of the land ice by the tides has raised a barrier of broken tables seventy-two feet wide and twenty feet high between the brig and islands. This action has caused a recession of the matin floc; our ressel has changed her position twenty feet within the last two spring tides, and the hawser comected with Butler Island parted with the strain." "The cutwater of the brig was then 280 feet from the margin of the ice. (Note of February th.)

The mean of all the soundings taken during the fourth series is very nearly fifteen feet, hence the constant index error, to refer the observations to the level previously adopted, is eight feet, which correction was applied, converting at the same time the record of fathoms into feet.

The following tidal record extends, therefore, over about nine and a half hunations between October 10, 1853, and October 22, 1854, during which interval the time and height of nearly five hundred high and as many low waters were secured.

\section{Record of the Observations of the Tides at Van Rensselaer IIarbor, North Greenland, in 1853, 1854, and 1855 . \\ Position of the Winter Quarters, \\ Latitude $7 S^{\circ} 37^{\prime}$ north, and longitude $70^{\circ} 53^{\prime}$, or $4^{\mathrm{h}} 43^{\mathrm{m}} .5$ west of Greenwich. ${ }^{1}$}

The first column for each day is copied from the original log-book, the second column contains the reduction to the adopted zero of scale found graphically as explained, and the third column contains the observations refered to the same mean level.

\footnotetext{
${ }^{3}$ See my discussion of the astronomical observations of the expedition in vol. XII of the Suithconian Contributions to Kinowledge, I860).
} 
Series 1.-Tidal Observations from October 10, 1853, to Deceniber 28, 1853.

Hourly obserrations on the pulley-gauge. Adopted reading of mean level 7.0, expressed in units of the scale. Incroasing numbers indicate rise of water.

\section{October: 1853}

\begin{tabular}{|c|c|c|c|c|c|c|c|c|c|c|c|c|c|c|c|c|c|c|}
\hline $\begin{array}{l}\text { ivean } \\
\text { solar } \\
\text { hour. }\end{array}$ & 10th. & $\begin{array}{c}\text { Red. } \\
\text { to } \\
\text { level. }\end{array}$ & $\begin{array}{l}\text { Ref. } \\
\text { obs. }\end{array}$ & 11th. & $\begin{array}{c}\text { Red. } \\
\text { to } \\
\text { level. }\end{array}$ & $\begin{array}{l}\text { Ref. } \\
\text { obs. }\end{array}$ & I th. & $\begin{array}{c}\text { Red. } \\
\text { to } \\
\text { level. }\end{array}$ & $\begin{array}{l}\text { Ref. } \\
\text { obs. }\end{array}$ & I3th. & $\begin{array}{c}\text { Red. } \\
\text { to } \\
\text { lerel. }\end{array}$ & $\begin{array}{l}\text { Ref. } \\
\text { obs. }\end{array}$ & 14th. & $\begin{array}{c}\text { Red. } \\
\text { to } \\
\text { level. }\end{array}$ & $\begin{array}{l}\text { Ref. } \\
\text { obs. }\end{array}$ & 17 th. & $\begin{array}{c}\text { Red. } \\
\text { to } \\
\text { level. }\end{array}$ & $\begin{array}{l}\text { Ref. } \\
\text { obs. }\end{array}$ \\
\hline 1 & - . & & & 5.4 & -1.0 & 4.4 & 5.0 & -1.0 & 4.0 & 7.5 & -1.3 & 6.2 & 7.0 & -1.5 & 5.5 & - . & & \\
\hline 2 & $6.6^{2}$ & -1.0 & 5.6 & & " & 4.4 & 5.0 & " & 4.0 & 6.4 & “ & 5.1 & 5.4 & $"$ & 3.9 & $\ldots$ & & \\
\hline 3 & 7.6 & " & 6.6 & 5.5 & " & 4.5 & 5.2 & " & 4.2 & 5.0 & " & 3.7 & 4.4 & “ & 2.9 & - . - & & - - \\
\hline 4 & 8.1 & " & 7.1 & 6.1 & " & 5. & 6.0 & “ & 5.0 & 5.5 & " & 4.2 & 4.2 & " & 2.7 & . . - & & - . . \\
\hline 5 & 8.7 & " & 7.7 & c. & t6 & 5.9 & 7.9 & " & 6.0 & 7.9 & "6 & 6.6 & 4.4 & -1.6 & 2.8 & $\ldots$ & & \\
\hline 6 & 6.7 & " & 7.7 & 7.3 & " & 6.3 & 8.5 & " & 7.5 & 8.3 & " & 7.0 & $5.5^{\circ}$ & " & 3.9 & - . - & & \\
\hline 7 & 8.7 & " & 7.8 & 7.7 & " & 6.7 & 8.9 & " & 7.9 & 8.7 & -1.4 & 7.3 & 7.5 & " & 5.3 & $\ldots$ & & ... \\
\hline 8 & 3.0 & "6 & 8.0 & 7.7 & "6 & 6.7 & 8.9 & " & 7.9 & 9.4 & " & 8.0 & 9.6 & " & 8.0 & - . - & & \\
\hline 9 & 6.4 & " & 5.4 & $7.6^{\circ}$ & $"$ & 6.6 & & & $\ldots$ & 10.5 & " & 9.1 & 11.2 & " & $9.6^{2}$ & $\ldots$ & & \\
\hline 10 & 5.9 & $"$ & 4.9 & 6.6 & " & 5.6 & 7.5 & -1.1 & 6.7 & 10.5 & " & 9.1 & 11.4 & " & 9.8 & - . - & & $\ldots$ \\
\hline 11 & 5.7 & " & 4. & 6.1 & " & 5.1 & 6.7 & " & 5.6 & 10.3 & "6 & 8.9 & 11.3 & " & 3.7 & - . - & & \\
\hline Noon & 5.8 & " & & 5.8 & "6 & & 5.6 & “" & 4.5 & 9.9 & " & 8.5 & 11.0 & -1.7 & 9.3 & $\ldots$ & & \\
\hline I & 6.7 & " & 5. & 5.8 & "6 & & 5.3 & " & 4.2 & 7.6 & " & 6.2 & 0.4 & 16 & 7.7 & . . - & & $\ldots$ \\
\hline 2 & 7.3 & " & 6.3 & 5.6 & " & 4.8 & 5.3 & " & 4.2 & 6.7 & " & 5.3 & 7.4 & " & 5.7 & $\ldots$ & & \\
\hline 3 & 8.8 & " & & 6. & " & & 5.3 & "6 & 4.2 & 5.6 & -1.5 & 4.1 & 6.6 & " & 4.9 & & & \\
\hline 4 & 9.3 & " & $\varepsilon$. & 7. & " & & 5.3 & " & 4.2 & 4. & " & 3.1 & 4.4 & & 2.7 & 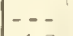 & & \\
\hline 5 & 10.2 & " & 9.2 & 3. & " & 8. & 6.4 & -1.2 & 5.2 & 6.8 & " & 5.0 & 4.6 & " & 2.9 & 4.2 & -2.7 & 1.5 \\
\hline 6 & 10.2 & " & & 10. & "6 & & 7.8 & $"$ & 6.6 & & " & .5 & 5.9 & "6 & 4.2 & 4.5 & $"$ & 1.8 \\
\hline 7 & 10.2 & ": & 9 & 10. & " & 3. & 9.8 & “ & 8.7 & 10.5 & " & 9.0 & 9.2 & " & 7.5 & & & \\
\hline s & 9.9 & $"$ & & 10. & " & & 11.0 & " & 9.8 & 11 & “ & 10.1 & 12.0 & -1.8 & 10.2 & 9.5 & " & 6.8 \\
\hline 9 & $\therefore$ & " & & $\because$. & if & & 11.3 & -1.3 & & & “ & .9 & & " & & 12.6 & "6 & 9.9 \\
\hline 10 & 7. & a & & 9.0 & " & 8. & 11.3 & 6 & 10.0 & I 2.4 & " & 10.9 & I3.I & "6 & 11.3 & 13.0 & 6 & 10.3 \\
\hline & 6.3 & " & 5.3 & 7.2 & $"$ & 6. & 9.7 & " & 8.4 & 12.4 & " & 10.8 & 13.1 & " & 11.3 & 13.4 & “ & 10.7 \\
\hline Midn't & 5.7 & " & 4.7 & 5.6 & " & 4.6 & 8.3 & " & 7.0 & 10.4 & " & 8.9 & 13.0 & -1.9 & 11.1 & 13.4 & "6 & 10.7 \\
\hline
\end{tabular}

October, 1853.

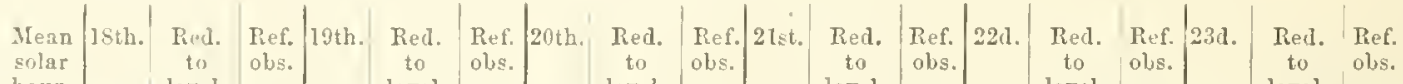

\begin{tabular}{|c|c|c|c|c|c|c|c|c|c|c|c|c|c|c|c|c|c|c|}
\hline $\begin{array}{l}\text { solar } \\
\text { hour. }\end{array}$ & & $\begin{array}{c}\text { to } \\
\text { level. }\end{array}$ & obs. & & $\begin{array}{l}\text { to } \\
\text { lerel. }\end{array}$ & olos. & & $\begin{array}{c}\text { to } \\
\text { lerel. }\end{array}$ & obs. & & $\begin{array}{c}\text { to } \\
\text { level. }\end{array}$ & obs. & & $\begin{array}{l}\text { to } \\
\text { level. }\end{array}$ & ols. & & $\begin{array}{l}\text { to } \\
\text { level. }\end{array}$ & olss. \\
\hline 1 & 11.5 & -2.7 & 8.8 & 13.6 & -2.2 & 11.4 & 13.8 & -3.3 & 11.5 & 11.6 & -1.7 & 9.9 & 10.7 & -0.2 & 10.5 & 7.0 & +0.8 & 7.8 \\
\hline 2 & 8.9 & "6 & (i.2 & 12.11 & " & 0.5 & 12.6 & "s & 10.3 & 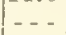 & " & $\ldots$ & 10.2 & "i & 10.0 & 8.0 & & 8. \\
\hline 3 & 6.6 & " & 4.1 & 8.9 & 6. & 6.7 & 111.5 & $" 6$ & 8.2 & 10.8 & -1.6 & 9.2 & 10.0 & -0.1 & 9.9 & 9.5 & $"$ & 10.3 \\
\hline 4 & 5.5 & " & 2.8 & 6.6 & " & 4.4 & 8.0 & " & 5.7 & 9.6 & -1.5 & 8.1 & 9.0 & " & 8.9) & 9.0 & +0.9 & 9.9 \\
\hline 5 & 4.4 & -2.6 & 1.8 & 4.5 & 16 & 2.3 & 7.9 & -2.2 & 5.7 & 6.7 & -1.4 & 5.3 & 8.0 & 0.0 & 8.0 & 6.8 & 6 & 7.8 \\
\hline 6 & 4.4 & "6 & 1.4 & 3.4 & " & 1.6 & 7.9 & " & 5.7 & 4.6 & "6 & 3.2 & 7.0 & " & 7.0 & 4.5 & " & 5.4 \\
\hline 7 & 6.5 & " & 3.8 & 3.8 & " & 1.6 & 7.9 & "6 & 5.7 & 4.1 & -1.3 & 2.8 & 5.0 & "6 & 5.0 & 3.4 & " & 4.3 \\
\hline 8 & 8.7 & "6 & 6.1 & 4.7 & ": & 2.5 & 8.9 & " & 6.7 & 4.1 & -1.2 & 2.9 & 5.11 & +0.1 & 5.1 & 3.0 & " & 3.9 \\
\hline 9 & 11.8 & -2.5 & 9.3 & 5.3 & 36 & 3.1 & $x .7$ & 16 & 6.5 & 6.7 & "6 & 5.5 & 3.3 & " & 3.4 & 3.1 & 6 & 4.0 \\
\hline 10) & $\ldots$ & & - . & 11.11 & " & 8.8 & 8.7 & $" 6$ & 6.5 & 8.5 & -1.1 & 7.4 & 4.5 & +0.2 & 4.7 & 3.5 & "6 & 4.4 \\
\hline 11 & - . . & & -- & 13.6 & " & 11.4 & 12.9 & "6 & 10.7 & 10.5 & 6 & 9.7 & 6.3 & " & 6.5 & 3.7 & " & 4.6 \\
\hline Noon & $\ldots$ & & $\cdots$ & 14.4 & " & 12.2 & 13.6 & " & .4 & 11.7 & -1.0 & 10.7 & 7.2 & " & 7.4 & 4.5 & " & 5.4 \\
\hline 1 & - . & & $\ldots$ & 14.6 & " & 12.4 & 14.0 & -2.1 & 11.6 & 11.8 & " & 10.9 & 7.0 & +0.3 & 7.3 & 6.5 & " & 7.4 \\
\hline 2 & 14.6 & " & 12.3 & 12.6 & "6 & 10.4 & $14 .(1$ & 6 & 11.9 & 11.9 & -0.9 & 11.0 & 7.5 & " & 7.5 & 7.0 & "6 & 7.9 \\
\hline 3 & 101.6 & -2.4 & 8.2 & 111.4 & $" 6$ & 8.2 & - - & & $\ldots$ & 11.9 & -0.8 & 11.1 & 9.5 & +0.4 & 9.9 & 8.5 & " & 9.4 \\
\hline 4 & $8.1 \%$ & $"$ " & (i.2 2 & $9.6 i$ & -2.3 & 7.3 & $-\cdots$ & & - - - & 11.8 & " & 11.0 & 9.2 & " & $9.1 \mathrm{i}$ & 9.5 & $"$ & 10.4 \\
\hline 5 & 6.6 & "6 & 4.2 & 6.6 & "6 & 4.3 & 7.7 & $" 6$ & 5.6 & 9.11 & -0.7 & 8.3 & 9.2 & 16 & 9.6 & 8.1) & $+(1.8$ & 8.8 \\
\hline 6; & 4.4 & 6 & 2.11 & 5.2 & $" 6$ & 2.9 & 6.2 & "6 & 4.1 & 7.5 & 6 & 6.8 & 6.7 & +0.5 & 7.2 & 7.4 & $" 6$ & 8.2 \\
\hline 7 & 4.4 & -2.3 & 2.1 & 4.2 & "6 & 1.9) & 5.5 & "s & & 5.5 & - 0.6 & 4.9 & 5.2 & " & 5.7 & 7.0 & " & 7.8 \\
\hline s & 5.5 & 6 & 3.2 & 4. & $"$ & 2.5 & 5.2 & -2.0 & 3.2 & 5.0 & 6 & 4.4 & 3.9 & +0.6 & 4.5 & 6.1 & " & 6.9 \\
\hline !) & 8.3 & "6 & 6.11 & di.k & $"$ " & .1 .5 & 4.7 & " & 2.7 & 5.0 & -0.5 & 4.5 & $3 . ?$ & " & 4.5 & 5.5 & "6 & $(3.3$ \\
\hline 111 & 10.4 & " & $\$ .1$ & !..4 & " & 7.1 & $0 . x$ & -1.8 & 4. $\}$ & 5.0 & " & 4.5 & 4.11 & & 4.6 & 4.5 & +11.7 & 5.2 \\
\hline 11 & $12.1 ;$ & " & 111.3 & $11.1 \%$ & "6 & $\$ 1.3$ & 9.8 & " & 7.8 & 5.0 & -11.4 & 4.6 & 6.3 & +11.7 & 7.0 & 5.0 & 4 & 5.7 \\
\hline $\operatorname{li}\left(\mathrm{n}^{4}\right.$ & 13.7 & -2.2 & 11.5 & 13.1 & $"$ & 11.1 & 11.0 & -1.8 & 3.2 & 5.0 & -11.3 & 4.7 & - - & & $\cdots$ & 5.5 & 6 & 6.2 \\
\hline
\end{tabular}

Joreular oljgervations commence Octolyer 10,2 A, M.

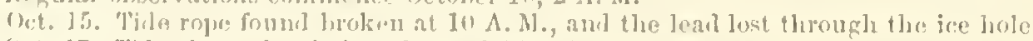

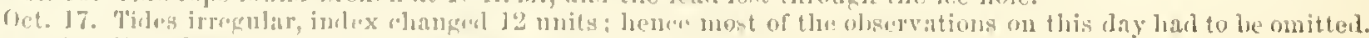

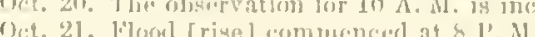

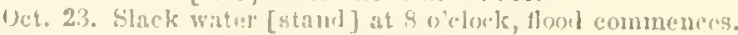


Surmis I.-Tidal Obsfrvations from October 10, 1853, to Decfamber 28, 1853. Ilourly observations on the pulley-gauge. Adopted reading of mean level 7.0, cxpressed in units of the seale. Increasing numbers iudieate rise of water.

\begin{tabular}{|c|c|c|c|c|c|c|c|c|c|c|c|c|c|c|c|c|c|c|}
\hline \multicolumn{19}{|c|}{ October, 1853.} \\
\hline $\begin{array}{l}\text { Mean } \\
\text { solar } \\
\text { bour. }\end{array}$ & 21 th. & $\begin{array}{c}\text { liel. } \\
\text { to } \\
\text { level. }\end{array}$ & $\begin{array}{l}\text { Ref. } \\
\text { obs. }\end{array}$ & & $\begin{array}{c}\text { Rerl. } \\
\text { to } \\
\text { level. }\end{array}$ & $\begin{array}{l}\text { Ref. } \\
\text { obs. }\end{array}$ & 26 th. & $\begin{array}{c}\text { Ted. } \\
\text { to } \\
\text { level. }\end{array}$ & $\begin{array}{c}\text { lief. } \\
\text { obs. }\end{array}$ & 27th. & $\begin{array}{c}\text { Rerl. } \\
\text { to } \\
\text { level. }\end{array}$ & $\begin{array}{l}\text { Ref. } \\
\text { ols. }\end{array}$ & $28 \mathrm{th}$. & $\begin{array}{c}\text { Red. } \\
\text { to } \\
\text { level. }\end{array}$ & $\begin{array}{l}\text { Ref. } \\
\text { obs. }\end{array}$ & 29 th. & $\begin{array}{c}\text { Rerl. } \\
\text { to } \\
\text { level. }\end{array}$ & $\begin{array}{l}\text { Ref. } \\
\text { olys. }\end{array}$ \\
\hline $\begin{array}{c}1 \\
3 \\
3 \\
4 \\
5 \\
6 \\
7 \\
8 \\
9 \\
10 \\
11 \\
\text { Noon } \\
1 \\
2 \\
3 \\
4 \\
5 \\
6 \\
7 \\
8 \\
9 \\
10 \\
11 \\
\text { Nidn't }\end{array}$ & \begin{tabular}{|c|}
6.5 \\
7.0 \\
8.11 \\
8.0 \\
7.3 \\
6.5 \\
5.4 \\
4.0 \\
5.0 \\
5.0 \\
5.0 \\
5.0 \\
7.7 \\
.-- \\
..- \\
-9.5 \\
9.5 \\
9.5 \\
8.1 \\
7.0 \\
6.0 \\
5.8 \\
5.5 \\
5.5
\end{tabular} & 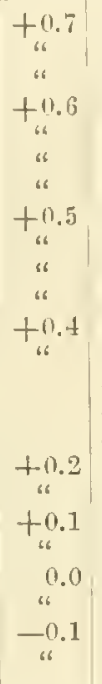 & \begin{tabular}{|c|}
7.2 \\
7.7 \\
8.7 \\
8.6 \\
7.9 \\
7.1 \\
6.0 \\
4.5 \\
5.5 \\
5.5 \\
5.5 \\
5.4 \\
8.1 \\
.- \\
$\ldots .$. \\
9.7 \\
.9 .7 \\
8.2 \\
7.1 \\
6.0 \\
5.8 \\
5.4 \\
5.4
\end{tabular} \mid & $\begin{array}{l}5.5 \\
5.5 \\
6.5 \\
7.2 \\
7.3 \\
7.0 \\
6.4 \\
5.6 \\
5.6 \\
5.6 \\
5.6 \\
5.6 \\
7.3 \\
6.3 \\
6.8 \\
7.5 \\
7.3 \\
7.6 \\
8.3 \\
8.5 \\
7.1 \\
6.1 \\
5.4 \\
5.0\end{array}$ & 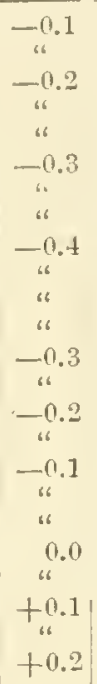 & $\begin{array}{l}5.4 \\
5.7 \\
6.3 \\
7.0 \\
7.1 \\
6.7 \\
6.1 \\
5.3 \\
5.2 \\
5.2 \\
5.2 \\
5.2 \\
7.0 \\
6.0 \\
6.6 \\
7.3 \\
7.2 \\
7.5 \\
8.2 \\
8.5 \\
7.1 \\
6.2 \\
5.5 \\
5.2\end{array}$ & \begin{tabular}{|l|}
5.0 \\
5.5 \\
5.5 \\
5.8 \\
5.8 \\
6.2 \\
6.5 \\
6.5 \\
6.1 \\
5.6 \\
5.5 \\
5.5 \\
5.5 \\
5.5 \\
6.0 \\
6.5 \\
7.5 \\
8.0 \\
8.2 \\
8.5 \\
8.5 \\
7.1 \\
6.1 \\
4.5
\end{tabular} & 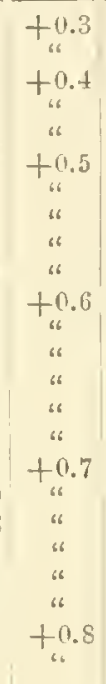 & \begin{tabular}{|l|}
5.3 \\
5.8 \\
5.9 \\
6.2 \\
6.2 \\
6.7 \\
7.0 \\
7.0 \\
6.6 \\
6.1 \\
6.1 \\
6.1 \\
6.1 \\
6.1 \\
6.6 \\
7.1 \\
8.2 \\
8.7 \\
8.9 \\
9.2 \\
9.2 \\
7.5 \\
6.9 \\
5.6
\end{tabular} & $\begin{array}{l}4.0 \\
4.0 \\
4.5 \\
5.0 \\
6.0 \\
6.8 \\
7.1 \\
7.1 \\
7.1 \\
6.0 \\
5.6 \\
4.5 \\
4.5 \\
5.0 \\
5.3 \\
6.3 \\
7.7 \\
7.3 \\
8.6 \\
8.7 \\
8.7 \\
7.1 \\
6.3 \\
5.9\end{array}$ & 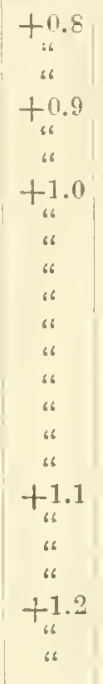 & \begin{tabular}{|c|}
4.8 \\
4.8 \\
5.3 \\
5.9 \\
6.9 \\
7.7 \\
8.1 \\
8.1 \\
8.1 \\
7.0 \\
6.6 \\
5.5 \\
5.5 \\
6.11 \\
6.3 \\
7.3 \\
8.7 \\
8.4 \\
9.7 \\
9.8 \\
9.8 \\
8.3 \\
7.5 \\
7.1 \\
\end{tabular} & \begin{tabular}{|c|}
4.5 \\
3.5 \\
3.4 \\
4.3 \\
5.3 \\
5.6 \\
7.3 \\
8.2 \\
\hdashline.. \\
.-- \\
.-- \\
..- \\
4.8 \\
4.3 \\
4.0 \\
4.0 \\
6.0 \\
7.1 \\
8.0 \\
9.4 \\
9.8 \\
9.8 \\
9.0 \\
7.3
\end{tabular} & $\begin{array}{c} \\
" 6 \\
" \\
" \\
" \\
" 6 \\
+1.0 \\
" 6 \\
\text { + } \\
+1.9\end{array}$ & \begin{tabular}{r|}
5.6 \\
4.6 \\
4.5 \\
5.4 \\
6.4 \\
6.7 \\
8.4 \\
8.3 \\
\hdashline.. \\
..- \\
..- \\
\hdashline 5.9 \\
5.4 \\
5.1 \\
5.1 \\
7.1 \\
8.2 \\
9.0 \\
10.4 \\
10.8 \\
10.8 \\
9.9 \\
8.2
\end{tabular} & $\begin{array}{r}5.1 \\
4.4 \\
2.5 \\
2.5 \\
3.11 \\
5.5 \\
7.0 \\
7.8 \\
9.8 \\
9.8 \\
9.6 \\
9.3 \\
6.8 \\
4.8 \\
4.0 \\
4.0 \\
4.0 \\
5.5 \\
6.7 \\
9.4 \\
9.5 \\
10.5 \\
10.5 \\
8.5\end{array}$ & 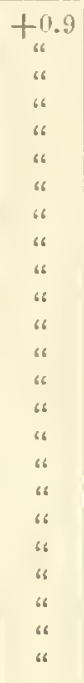 & $\begin{array}{r}6.0 \\
5.3 \\
3.7 \\
3.4 \\
3.9 \\
6.4 \\
7.9 \\
8.7 \\
10.7 \\
10.7 \\
111.5 \\
10.2 \\
7.7 \\
5.7 \\
4.6 \\
4.9 \\
4.9 \\
6.4 \\
7.4 \\
0.9 \\
10.4 \\
11.4 \\
11.4 \\
9.4\end{array}$ \\
\hline \multicolumn{7}{|c|}{ October, 1853.} & \multicolumn{12}{|c|}{ November, 1853.} \\
\hline $\begin{array}{l}\text { Mean } \\
\text { solar } \\
\text { hour. }\end{array}$ & 30 th. & $\begin{array}{c}\text { Red. } \\
\text { to } \\
\text { lerel. }\end{array}$ & \begin{tabular}{l|l} 
Ref. & 3 \\
obs. &
\end{tabular} & 31st. & $\begin{array}{c}\text { Red. } \\
\text { to } \\
\text { level. }\end{array}$ & $\begin{array}{l}\text { Ref. } \\
\text { obs. }\end{array}$ & 1 st. & $\begin{array}{c}\text { Red. } \\
\text { to } \\
\text { le rel. }\end{array}$ & $\begin{array}{l}\text { Pef. } \\
\text { obs. }\end{array}$ & $2 \mathrm{~d}$. & $\begin{array}{c}\text { Red. } \\
\text { to } \\
\text { level. }\end{array}$ & $\begin{array}{l}\text { Ref. } \\
\text { obs. }\end{array}$ & $3 \mathrm{~d}$. & $\begin{array}{c}\text { Red. } \\
\text { to } \\
\text { level. }\end{array}$ & $\begin{array}{l}\text { Ref. } \\
\text { obs. }\end{array}$ & 4th. & $\begin{array}{c}\text { Red. } \\
\text { to } \\
\text { level. }\end{array}$ & $\begin{array}{l}\text { Ref. } \\
\text { obs. }\end{array}$ \\
\hline 1 & 4.5 & +0.9 & 5.4 & 5.4 & +1.5 & 6.9 & 6.8 & +1.7 & 8.5 & 0.1 & +1.4 & 10.51 & 13.5 & $-1.7]$ & $11.8 / 1$ & 13.0 & -1.4 & 11.6 \\
\hline 2 & 3.5 & 80 & 4.4 & 3.8 & & 5.3 & 4.0 & " & 5.7 & 8.5 & +1.3 & 10.11 & 12.0 & $" 1$ & 10.31 & 12.5 & $" 6$ & 11.1 \\
\hline 3 & 2.0 & " & 3.9 & 3.0 & +1.6 & 4.6 & 2.5 & " & 4.2 & 7.1 & +1.2 & 8.3 & 9.0 & " & 7.31 & 11.2 & $"$ & 9.8 \\
\hline 4 & 2.0 & " & 2.9 & 0.0 & 6 & 1.6 & 1.0 & “ & 2.7 & 4.7 & +1.0 & 5.7 & 6.7 & -1.6 & 5.1 & 9.5 & $"$ & s \\
\hline 5 & 3.2 & “ & 4.1 & 1.0 & " & 2.6 & 1.0 & “ & 2.7 & 1.5 & $1+0.7$ & 2.2 & 3.7 & " & 2.1 & 6.0 & " & \\
\hline 6 & 4.2 & " & 5.1 & 1.2 & $"$ & 2.8 & 2.2 & " & 3.9 & 0.5 & +0.5 & 1.0 & 1.8 & " & 0.2 & 4.4 & " & 3.0 \\
\hline 7 & 6.2 & +1.0 & 7.2 & 3.1 & +1.7 & 4.8 & 3.2 & " & 4.9 & 0.7 & +0.2 & 0.9 & 1.5 & -1.5 & 0.0 & 2.0 & “" & 0.6 \\
\hline 8 & 8.5 & " & 9.5 & 6.5 & " & 8.2 & 7.0 & “ & 8.7 & 3.0 & 0.11 & 3.0 & 2.4 & “ & 0.9 & 1.7 & " & $(1.3$ \\
\hline 9 & 10.1 & +1.11 & 11.2 & 8.5 & " & 111.2 & 9.5 & +1.6 & 11.1 & 7.2 & -1.2 & 7.0 & 3. & $"$ & 1.9 & 3.7 & " & 2.3 \\
\hline 10 & 10.4 & " 1 & 11.5 & 9.3 & " & 11.0 & 10.5 & " & 12.1 & 0.8 & -0.5 & 9.3 & 4. & " & 2.7 & & " & 5.9 \\
\hline 11 & 10.4 & " 1 & 11.51 & 10.3 & " & 12.13 & 10.7 & " & 12.31 & 10.9 & -0.7 & 10.2 & 6. & " & $\begin{array}{r}4.5 \\
1\end{array}$ & 10.5 & " & 9.1 \\
\hline Noon & 10.4 & +1.21 & 11.6 & 10.0 & $"$ & 11.7 & 111.7 & $"$ & 12.31 & 15.3 & -1.0 & 14.3 & \begin{tabular}{|l|}
6.7 \\
\end{tabular} & “ & 5.21 & 12.6 & 4 & 11.2 \\
\hline 1 & 8.2 & " & 9.4 & 7.5 & " & 9.2 & 111.2 & " & 11.81 & 15.2 & -1.21 & 14.01 & 15.7 & " & 14.2 & $\ldots$ & & $\ldots$ \\
\hline 2 & 5.5 & “ & 6.7 & 5.2 & “ & 6.9 & 9.2 & “ & 10.81 & $13.6^{3}$ & -1.5 & 12.11 & 15.2 & " & & -- & & $\cdots$ \\
\hline 3 & 3.7 & " & 4.9 & 3.7 & “ & 5.4 & 8.1 & “ & $: 9.7$ & 10.5 & -1.6 & S.9 1 & 12.5 & " & $\left.11.0\right|_{1} ^{-}$ & 12.0 & “ & \\
\hline 4 & 3.4 & $"$ & 4.6 & 0.1 & $"$ & 1.8 & 4.5 & +1.5 & 6.0 & 6.8 & -1.7 & \begin{tabular}{l|l|}
5.1 & 1
\end{tabular} & 10.1 & " & S.6 1 & 11.5 & " & 111.1 \\
\hline 5 & 2.6 & +1.3 & 3.9 & 0.21 & “ & 1.9 & 3.11 & $"$ & 4.5 & 3.8 & 4 & $\because .1$ & 6.5 & $"$ & 5.0 & & "6 & \\
\hline 6 & 3.4 & " & 4.7 & 1.2 & “ & 2.0 & 3.3 & “ & 3.8 & 2.11 & $"$ & 0.3 & 4.5 & " & 3.11 & & “: & \\
\hline 7 & 5.1 & 16 & 6.4 & 3.0. & " & 4.7 & 2.1 & " & 3.6 & 1.8 & $"$ & 0.1 & 3.5 & " & 2.0 & $\begin{array}{l}3.2 \\
3.0\end{array}$ & “" & $\begin{array}{l}1.8 \\
1.15\end{array}$ \\
\hline 8 & 6.8 & “" & 8.1 & 5.2 & " & 6.9 & 4.0 & " & 5.5 & 3.2 & “ & 1.5 & $\begin{array}{l}3.5 \\
3.1\end{array}$ & “" & $\begin{array}{l}2.0 \\
1.5\end{array}$ & 3.0 & “ & 1.6 \\
\hline 9 & 9.5 & +1.4 & 10.9 & 8.1 & " & 9.8 & 9.0 & “ & 10.5 & 3.3 & “" & 1.6 & 4.5 & “ & 3.10 & 3.1 & “" & 1.7 \\
\hline 10 & 110.3 & ". & 11.7 & 9.5 & $"$ & 11.2 & 10.1 & $"$ & 11.6 & 3.3 & “ & 1.1; & 7.3 & “ & 5.8 & 5.11 & " & \\
\hline 11 & 10.3 & " & 11.7 & 9.5 & " & 11.2 & 10.1 & " & 11.6 & 3.3 & " & 1.6 & 9.3 & " & & 7.5 & " & \\
\hline Mictn't & 9.0 & +1.51 & 111.51 & 11.5 & " & 12.21 & 10.1 & " & 11.6 & 3.3 & “ & 1.151 & & " & 10.5 & 0.5 & $"$ & 8.4 \\
\hline $\begin{array}{l}\text { Nor. } \\
\text { ar li }\end{array}$ & rot & $\begin{array}{l}\text { for. } 6 . \\
\text { ors wa }\end{array}$ & Be & thee & es & & th & 8 & 4 & & $1: t$ & & & $s$ & & & 5 & \\
\hline
\end{tabular}


Series I.-Tidal Observations from October 10, 1853, to December 2S, 1853.

Hourly observations on the pulley-gauge. Adopted reading of mean level 7.0, expressed in units of the scale. Increasing numbers indicate rise of water.

November, 1853.

\begin{tabular}{|c|c|c|c|c|c|c|c|c|c|c|c|c|c|c|c|c|c|c|}
\hline $\begin{array}{l}\text { Mean } \\
\text { solar } \\
\text { hour. }\end{array}$ & sth. & $\begin{array}{l}\text { Red. } \\
\text { to } \\
\text { level. }\end{array}$ & $\begin{array}{l}\text { Ref. } \\
\text { obs. }\end{array}$ & 6th. & $\begin{array}{c}\text { Red. } \\
\text { to } \\
\text { level. }\end{array}$ & $\begin{array}{l}\text { Ref. } \\
\text { obs. }\end{array}$ & Tth. & $\begin{array}{c}\text { Red. } \\
\text { to } \\
\text { level. }\end{array}$ & $\begin{array}{l}\text { Ref. } \\
\text { obs. }\end{array}$ & Sth. & $\begin{array}{c}\text { Rerl. } \\
\text { to } \\
\text { lerel. }\end{array}$ & $\begin{array}{l}\text { Ref. } \\
\text { obs. }\end{array}$ & 9th. & $\begin{array}{c}\text { Red. } \\
\text { to } \\
\text { level. }\end{array}$ & $\begin{array}{l}\text { Ref. } \\
\text { obs. }\end{array}$ & 10th & $\begin{array}{c}\text { Red. } \\
\text { to } \\
\text { level. }\end{array}$ & $\begin{array}{l}\text { Fief. } \\
\text { obs. }\end{array}$ \\
\hline \multirow{3}{*}{1} & \multirow{3}{*}{11.5} & \multirow{3}{*}{-1.4} & \multirow{3}{*}{10.1} & \multirow{3}{*}{9.0} & \multirow{3}{*}{-1.0} & \multirow{3}{*}{8.0} & \multirow{3}{*}{6.6} & \multirow{3}{*}{-0.6} & \multirow{3}{*}{6.0} & 4.3 & \multirow{3}{*}{-0.5} & \multirow{3}{*}{$\begin{array}{l}3.8 \\
4.8 \\
5.1\end{array}$} & \multirow{3}{*}{$\begin{array}{l}3.8 \\
3.9 \\
4.2\end{array}$} & \multirow{3}{*}{$\begin{array}{c}+0.2 \\
6 \\
6\end{array}$} & \multirow{3}{*}{$\begin{array}{l}4.0 \\
4.1 \\
4.4\end{array}$} & \multirow{3}{*}{$\begin{array}{l}4.5 \\
4.5 \\
4.3\end{array}$} & \multirow{2}{*}{\multicolumn{2}{|c|}{$\begin{array}{l}4.3 \\
4.3\end{array}$}} \\
\hline & & & & & & & & & & 5.3 & & & & & & & & \\
\hline & & & & & & & & & & 5.6 & & & & & & & " & 4.1 \\
\hline 2 & 11.0 & " & 9.6 & 9.8 & " & 8.8 & 7.9 & " & 7.3 & E.2 & u & 5.7 & 4.0 & " & 4.2 & 4.0 & $"$ & 3.8 \\
\hline & & & & 9.9 & " & 8.9 & S. 5 & $"$ & 8.2 & & & & 4.5 & " & 4.7 & 4.2 & " & .0 \\
\hline 3 & 10.0 & $"$ & 8.6 & 10.0 & $"$ & 9.0 & 8.8 & $"$ & 8.2 & 7.1 & "6 & 6.6 & 5.0 & " & 5.2 & 4.5 & -0.3 & .2 \\
\hline & & & & 10.0 & $"$ & 9.0 & 8.8 & " & 8.2 & & & & & & & 4.5 & $"$ & .2 \\
\hline 4 & 9.2 & -1.3 & 7.9 & 10.0 & -0.9 & 9.1 & 8.5 & 64 & $8 \cdot 2$ & 7.9 & "6 & 7.4 & 6.0 & " & 6.2 & 4.1 & $"$ & .8 \\
\hline & & & & 5.1 & " & 7.2 & 5.8 & $"$ & 8.2 & 7.9 & -0.4 & 7.5 & & & & 5.8 & "6 & .5 \\
\hline 5 & 5.7 & ". & 4.4 & 7.7 & " & 6.8 & 8.6 & $" 4$ & 8.0 & 8.2 & " & 7.8 & 7.5 & $" 4$ & 7.7 & 6.5 & " & 0.2 \\
\hline 6 & 3.5 & " & 2.2 & 5.5 & $" 6$ & 4.6 & 7.8 & -0.5 & 7.3 & $\begin{array}{l}.0 .0 \\
7.8\end{array}$ & a & 7.4 & 7.9 & $" 4$ & 8.1 & 8.5 & " & 8.2 \\
\hline & & & & & & & & & & & & & 7.9 & "“ & 8.1 & 9.9 & 64 & .6 \\
\hline 7 & 2.3 & " & 1.0 & 4.5 & $"$ & 3.6 & 6.5 & " & 6.0 & 7.3 & -0.3 & 7.0 & 8.0 & +0.1 & 8.1 & 9.5 & $"$ & $\therefore$ \\
\hline & & & & 4.0 & $"$ & 3.1 & & & & & & & 8.0 & & 8.1 & 10.0 & "6 & 7 \\
\hline 8 & 2.0 & $"$ & 0.7 & 3.6 & -0.8 & 2.5 & 5.4 & " & 4.9 & 6.3 & " & 6.0 & 8.11 & "6 & 8.1 & 10.2 & -0.4 & 7.8 \\
\hline & 2.01 & $"$ & 0.7 & 3.1 & $"$ & 2.3 & & & & & & & 7.4 & " & 7.5 & 10.2 & 66 & .8 \\
\hline 9 & 2.4 & $"$ & 1.1 & 3.1 & " & 2.3 & 4.0 & $"$ & 3.5 & 5.5 & “6 & 5.2 & 6.9 & $"$ & 7.0 & 10.2 & "6 & .8 \\
\hline & 2.6 & 6 & 1.3 & 3.2 & " & 2.4 & 4.0 & " & 3.5 & & & & & & & 10.1 & $"$ & .7 \\
\hline 10 & 2.7 & -1.2 & 1.5 & 4.3 & $"$ & 3.5 & 3.7 & " & 3.2 & 5.3 & $" 6$ & 5.0 & 6.0 & " & 6.1 & 9.8 & $"$ & 9.4 \\
\hline & & & & & & & 3.9 & $"$ & 3.4 & & & & & & & & & \\
\hline 11 & $6 . \bar{T}$ & " & 5.5 & 5.7 & -0.7 & 5.0 & 4.4 & $"$ " & 3.9 & $\begin{array}{l}5.5 \\
5.3\end{array}$ & " & 5.2 & 4.8 & " & 4.9 & 9.0 & " & 8.6 \\
\hline Noon & 11.1 & $"$ & 9.9 & 8.3 & $"$ & 7.6 & 6.5 & $" 4$ & 6.3 & 5.1 & 16 & 4.9 & 4.6 & 0.0 & 4.6 & 7.5 & -0.5 & 7.0 \\
\hline & & & & & & & & & & 5.3 & " & 5.1 & & & & & & \\
\hline 1 & 13.1 & “" & 11.9 & $\ldots$ & & & 5.1 & $"$ " & 7.6 & 5.6 & $"$ & 5.4 & 4.4 & 16 & 4.4 & 6.5 & " & 6.0 \\
\hline & 13.6 & " & .4 & & & & & & & & & & 4. & & 4.5 & 6.3 & "، & 5.8 \\
\hline 2 & 14.2 & $"$ & 13.0 & $\cdots$ & & & 9.5 & $"$ " & 9.0 & 7.1 & "6 & 6.9 & 4.5 & " & 4.5 & 6.0 & 4 & 5.5 \\
\hline & 14.2 & " & & & & & & & & & & & 5.1 & $" 6$ & 5.1 & 6.0 & $"$ & 5.5 \\
\hline 3 & 14.1 & -1.1 & 13.0 & - - & & & 10.6 & $" 6$ & 10.1 & 8.3 & 16 & 8.1 & 5.5 & $"$ & 5.5 & 6.5 & -0.6 & 5.9 \\
\hline & 13.0 & " & 11.9 & & & & 10.9 & " & 10.4 & & & & & & & & & \\
\hline 4 & 12.3 & "t & 11.2 & -- & & & 11.2 & " & 10.7 & 9.5 & 16 & 9.3 & 7.1 & " & 7.1 & 7.0 & "، & 6.4 \\
\hline 5 & 10.8 & $" 6$ & 9.7 & $\begin{array}{l}11.0 \\
11.0\end{array}$ & -0.6 & $\begin{array}{l}10 . .9 \\
10.4\end{array}$ & $\begin{array}{l}11.2 \\
11.1\end{array}$ & " & $\begin{array}{l}10.7 \\
10.6\end{array}$ & 10.1 & -0.1 & 10.0 & 9.2 & -0. & 9.1 & 7.9 & " & 7.3 \\
\hline & & & & 10.7 & " & 10.1 & 10.2 & 6 & 9.7 & 10. & 16 & 10.4 & 9.6 & & 9.5 & & & \\
\hline 6 & 8.2 & " & 7.1 & 9.1 & " & 8.5 & 10.2 & " & 9.7 & 10.5 & "t & 10.4 & 10.1 & " & 111.0 & 9.5 & " & 8.9 \\
\hline & & & & & & & & & & & 4 & & 9.5 & & 9.4 & & & \\
\hline 7 & 5.1 & " & 4.0 & 7.5 & " & 6.9 & 8.5 & "، & 8.0 & 10.5 & " & 111.4 & 11.1 & " & 11.0 & 10.9 & " & 10.3 \\
\hline & & & & & & & & & & 10.5 & " & 10.4 & 11.1 & & & 11.4 & (6) & 0.8 \\
\hline 8 & 3.3 & " & 2.2 & 5.4 & "t & 4.8 & 7.5 & "4 & 7.0 & 10.0 & 0.0 & 10.11 & 11.0 & "6 & 10.9 & 11.0 & "6 & 10.4 \\
\hline & 2.9 & $"$ & 1.8 & & & & & & & & & & 9. & & 9.7 & 110 & & 10.4 \\
\hline 9 & 3.11 & " & 1.9 & 42 & " & 3.6 & 5.6 & " & 5.1 & 8.1 & ": & 8.1 & 8.9 & " & 8.8 & 11.0 & " & 10.4 \\
\hline & 3.5 & "f & 2.4 & 4.11 & s6 & 3.4 & & & & & & & & & & 11.3 & u & 10.7 \\
\hline 10 & 4.1 & -1.0 & 3.1 & 3.2 & " & 2.6 & 4.4 & "4 & 3.9 & 7.0 & +0.1 & 7.1 & 7.0 & " & 6.9 & 11.3 & -0.7 & 10.6 \\
\hline & & & & 3.2 & 6 & 2.6 & 4.3 & " & 3.8 & & & & & & & 10.9 & " & 10.2 \\
\hline 11 & 6.11 & " & 5.0 & 4.0 & $"$ & 3.4 & 4.1 & $" 6$ & 3.6 & 4.9 & " & 5.0 & 4.7 & -0.2 & 4.5 & 9.5 & $"$ & 8.8 \\
\hline & & & & 4.5 & "6 & 3.9 & 4.1 & "6 & 3.6 & 42 & 4 & 4.3 & 4.7 & & 4.5 & & & \\
\hline Mirln't & 7.3 & $" 6$ & 6.3 & 4.9 & $"$ & 4.3 & 4.0 & $"$ & 3.5 & 4.0 & +0.2 & 4.2 & 4.5 & " & 4.3 & 8.5 & $"$ & 7.8 \\
\hline
\end{tabular}

Nor. R. From this date the olservations are half-hourly ; in the ahove reorl, however, only those halfhourly rosulings were ins.rted, which orent near a ligh or low water. 
Series I.-Thmal Opservations fuom Octomer 10, 1853 , to Decenber 2S, 1853.

Hourly observations on the pulley-gauge. Adopted reading of mean level 7.0, cxpressed in units of the scale. Inereasing numbers indicate rise of water.

\section{November, 1853.}

\begin{tabular}{|c|c|c|c|c|c|c|c|c|c|c|c|c|c|c|c|c|c|c|}
\hline $\begin{array}{l}\text { Mean } \\
\text { solar } \\
\text { hour. }\end{array}$ & Irth. & $\begin{array}{c}\text { Red. } \\
\text { to } \\
\text { level. }\end{array}$ & $\begin{array}{l}\text { Ref. } \\
\text { ubs. }\end{array}$ & $12 \mathrm{ch}$. & $\begin{array}{c}\text { Red. } \\
\text { to } \\
\text { level. }\end{array}$ & $\begin{array}{l}\text { Ref. } \\
\text { obs. }\end{array}$ & 13 th. & $\begin{array}{c}\text { Rerl. } \\
\text { to } \\
\text { level. }\end{array}$ & $\begin{array}{l}\text { Ref. } \\
\text { obs. }\end{array}$ & 14 th. & $\begin{array}{c}\text { Red. } \\
\text { to } \\
\text { level. }\end{array}$ & $\begin{array}{l}\text { Ref. } \\
\text { abs. }\end{array}$ & 15 th. & $\begin{array}{c}\text { Red. } \\
\text { to } \\
\text { level. }\end{array}$ & $\begin{array}{l}\text { Ref. } \\
\text { obs. }\end{array}$ & 16 th. & $\begin{array}{c}\text { Redl. } \\
\text { to } \\
\text { level. }\end{array}$ & $\begin{array}{l}\text { lief. } \\
\text { obs. }\end{array}$ \\
\hline \multirow[b]{2}{*}{1} & 6.1 & -0.7 & 5.4 & & & & & & & & & & & & & 10.8 & -5.5 & \\
\hline & 4.6 & " & 3.9 & 6.0 & -0.9 & 5.1 & 8.8 & -1.6 & 7.2 & 11.5 & -4.1 & 7.4 & 13.0 & -5.2 & 7.8 & 15.1 & & 9.6 \\
\hline \multirow[t]{2}{*}{2} & 3.6 & -0.8 & 2.8 & 5.5 & " & 4.6 & 6.7 & " & 5.1 & 9.1 & -4.2 & 4.9 & 10.0 & " & 4.8 & 14.7 & -5.6 & \\
\hline & 3.1 & "I & 2.3 & 4.5 & $"$ & 3.6 & & & & & & & & & & & & \\
\hline \multirow[t]{2}{*}{3} & 3.5 & " & 2.7 & 4.0 & -1.0 & 3.0 & 4.9 & -1.7 & 3.2 & 7.5 & -4.3 & 3.2 & 8.0 & " & 2.8 & 13.0 & " & 7.4 \\
\hline & 3.6 & " & 2.8 & 4.0 & "4 & 3.0 & 4.6 & & 2.9 & 7.0 & " & 2.7 & & & & & & \\
\hline \multirow[t]{2}{*}{4} & 4.1 & $"$ & 3.3 & 3.8 & " & 2.8 & 4.4 & -1.8 & 2.6 & 6.5 & -4.4 & 2.1 & 6.6 & " & 1.4 & 10.5 & " & \\
\hline & & & & 4.1 & st & 3.1 & 4.5 & " & 2.7 & 6.5 & " & 2.1 & 6.0 & " & 0.5 & 6.1 & -5.7 & 4 \\
\hline \multirow[t]{2}{*}{5} & 5.0 & “ & 4.2 & 4.6 & " & 3.6 & 4.5 & -1.9 & 2.6 & 6.5 & $"$ & 2.1 & 5.9 & " & 0.7 & 6.1 & “ & $0^{-}$ \\
\hline & & & & & & & 5.0 & & 3.1 & 6.5 & " & 2.1 & 5.9 & “ & 0.7 & 6.3 & -5.8 & \\
\hline 6 & 5.6 & " & 4.8 & 7.1 & " & 6.1 & 5.5 & 16 & 3.6 & 7.0 & -4.5 & 2.5 & 6.2 & " & 1.0 & 6.3 & " & .5 \\
\hline \multirow[t]{2}{*}{7} & 8.0 & " & 7.2 & 8.6 & " & 7.6 & 7.5 & -2.0 & 5.5 & 9.0 & " & 4.5 & 8.0 & $"$ & 2.8 & 7.3 & -5.9 & 1.4 \\
\hline & 8.3 & $"$ & 7.5 & & & & & & & & & & & & & & & \\
\hline \multirow[t]{2}{*}{8} & 9.5 & " & 8.7 & 10.5 & "* & 9.5 & 10.2 & -2.1 & 8.1 & 12.4 & -4.6 & 7.8 & 10.5 & $"$ & 5.3 & 10.0 & -6.0 & 4.0 \\
\hline & 10.3 & " & 9.5 & 11.5 & $"$ & 10.5 & & & & & & & & & & & & \\
\hline \multirow[t]{2}{*}{9} & 111.3 & "6 & 9.5 & 12.0 & " & 11.0 & 12.7 & $"$ & 10.6 & 15.5 & " & 10.9 & $12 . ?$ & " & 7.7 & 12.7 & " & U. \\
\hline & 10.0 & " & 9.2 & 12.3 & " & 11.2 & 13.0 & -2.2 & 10.8 & & & & & & & & & \\
\hline 10 & 10.0 & $" 6$ & 9.2 & 12.2 & " & 11.2 & 13.7 & " & 11.5 & 17.0 & -4.7 & 12.3 & 16.0 & $" 6$ & 10.8 & 16.4 & -6.1 & 10. \\
\hline \multirow{2}{*}{11} & & 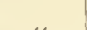 & & 12.0 & 16 & 11.0 & 13.7 & -2.3 & 11.4 & 17.5 & -4.8 & 12.7 & 17.3 & $"$ & 12.1 & & "6 & \\
\hline & 9.5 & "6 & 8.1 & 11.7 & -1.1 & 10.6 & $\mid \begin{array}{l}13.5 \\
12.9\end{array}$ & " & $\begin{array}{l}11.2 \\
10.6\end{array}$ & $\begin{array}{l}17.3 \\
17.1\end{array}$ & $\begin{array}{c}-4.9 \\
16\end{array}$ & $\begin{array}{l}12.4 \\
12.2\end{array}$ & $\begin{array}{l}18.5 \\
18.5\end{array}$ & $" 6$ & $\begin{array}{l}13.3 \\
13.3\end{array}$ & $\begin{array}{l}17.8 \\
18.5\end{array}$ & -6.2 & 12. \\
\hline Noon & 7.5 & " & 6.7 & 9.0 & " & 7.9 & 12.1 & -2.4 & 9.7 & 16.4 & -5.0 & 11.4 & 18.3 & -5.3 & 13.0 & 19.9 & -6.3 & 13.6 \\
\hline 1 & & & & & & & & & & & & & & " & 12.7 & 19.9 & "، & \\
\hline 1 & 6.0 & $" 6$ & 5.2 & 8.0 & "6 & 6.9 & 11.1 & -2.5 & 8.6 & 14.1 & $"$ & 8.1 & 17.6 & "، & 12.3 & 18.7 & -6.4 & 3 \\
\hline \multirow[t]{2}{*}{2} & 5.3 & $" 6$ & 4.5 & 6.3 & " & 5.2 & 9.1 & -2.6 & 6.5 & 12.0 & " & 7.0 & 15.5 & " & 10.2 & 17.9 & "6 & 11.5 \\
\hline & 5.3 & " & 4.5 & 5.6 & " & 4.5 & & & & & & & & & & & & \\
\hline \multirow[t]{2}{*}{3} & 5.0 & $"$ & 4.2 & 4.9 & $" 6$ & 3.8 & 7.5 & -2.7 & 5.1 & 9.3 & -5.1 & 4.2 & 12.0 & " & 6.7 & 15.0 & "6 & \\
\hline & 5.0 & $" 6$ & 4.2 & 5.0 & " & 3.9 & & & & & & & & & & (1) & $f<$ & \\
\hline 4 & 5.5 & "f & 4.7 & $\begin{array}{l}5.0 \\
5.0\end{array}$ & "6 & 3.9 & 6.3 & -2.8 & 3.5 & $\begin{array}{l}8.5 \\
9.0\end{array}$ & "6 & $\begin{array}{l}3.4 \\
3.9\end{array}$ & $\begin{array}{r}10.3 \\
9.5\end{array}$ & $" 6$ & 4.2 & 1200 & -6.3 & \\
\hline \multirow[t]{2}{*}{5} & 6.6 & " & 5.5 & 5.5 & -1.2 & 4.3 & 6.3 & -2.9 & 3.4 & 8.0 & " & 2.9 & 8.9 & " & 3.6 & 10.6 & " & 1.1 \\
\hline & & & & & & & 5.5 & & 2.6 & 8.0 & $"$ & 2.9 & 8.9 & ": & 3.6 & 10.3 & -6.7 & \\
\hline \multirow[t]{2}{*}{6} & 8.0 & " & 7.2 & 6.4 & " & 5.2 & 5.5 & -3.0 & 2.5 & 8.5 & -5.2 & 3.3 & 8.9 & -5.4 & 3.5 & 10.0 & " & \\
\hline & & & & & & & 6.8 & -3.1 & 3.7 & & & & 8.9 & $"$ & 3.5 & 10.0 & -6.8 & \\
\hline 7 & 10.0 & " & 9.2 & 7.7 & " & 6.5 & 8.7 & -3.2 & 5.5 & 9.6 & " & 4.4 & 9.2 & " & 3.8 & 10.0 & -6.9 & \\
\hline \multirow[t]{2}{*}{8} & 11.4 & -0.9 & 10.5 & 10.4 & -1.3 & 9.1 & 11.0 & -3.3 & 7.7 & 11.4 & " & 6.2 & 10.7 & " & 5.3 & 11.5 & -7.0 & 4.5 \\
\hline & & $"$ & 10.9 & & & & 137 & g & 102 & 131 & 66 & 79 & 7127 & "6 & 83 & 110 & "6 & \\
\hline$n$ & $\begin{array}{l}12.7 \\
12.7\end{array}$ & " & $\begin{array}{l}11.8 \\
11.5\end{array}$ & $\begin{array}{l}11.8 \\
12.5\end{array}$ & " & $\begin{array}{l}10.5 \\
11.5\end{array}$ & 13.1 & & 10.3 & 13.1 & & 8.9 & 10.6 & & 0.0 & 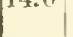 & & \\
\hline 10 & 12.1 & 16 & 11.2 & 12.7 & -1.4 & 11.3 & 14.4 & -3.6 & 10.8 & 14.7 & $"$ & 9.5 & 16.6 & " & 11.2 & 16.0 & " & 9. \\
\hline & & & & 12.5 & " & 11.1 & 14.5 & -3.7 & 10.8 & 15.0 & " & ก.8 & & & & & & \\
\hline 11 & 11.4 & " & 10.5 & 11.9 & " & 10.5 & 14.8 & -3.5 & 11.0 & 14.6 & " & 9.4 & 17.8 & " & 12.4 & 17.5 & -7.1 & 10.4 \\
\hline & & & & & & & 14.4 & -3.9 & 10.5 & 14.5 & " & 9.3 & 18.5 & -5.5 & 13.0 & 18.0 & “ & 10. \\
\hline Midn't & 9.2 & " & 8.3 & 10.6 & -1.5 & 9.1 & 13.6 & -4.0 & 9.6 & 14.1 & "6 & 8.9 & 18.5 & $" 6$ & 13.11 & 17.2 & "“ & 50 \\
\hline
\end{tabular}


Series I.-Tidal Observations from October 10, 1853, to Deceyiber 28, 1853.

IIourly observations on the pulley-gange. Adopted reading of mean level 7.0, expressed in units of the seale. Increasing numbers indicate rise of water.

November, 1853.

\begin{tabular}{|c|c|c|c|c|c|c|c|c|c|c|c|c|c|c|c|c|c|c|}
\hline $\begin{array}{l}\text { Mean } \\
\text { solar } \\
\text { hour. }\end{array}$ & 17 th. & $\begin{array}{c}\text { Tierl. } \\
\text { to } \\
\text { level. }\end{array}$ & $\begin{array}{l}\text { Ref. } \\
\text { olys. }\end{array}$ & 1 sth. & $\begin{array}{c}\text { Red. } \\
\text { to } \\
\text { level. }\end{array}$ & $\begin{array}{l}\text { Ref. } \\
\text { obs. }\end{array}$ & 19 th. & $\begin{array}{c}\text { Rerl. } \\
\text { to } \\
\text { level. }\end{array}$ & $\begin{array}{l}\text { Ref. } \\
\text { obs. }\end{array}$ & 20 th. & $\begin{array}{c}\text { Red. } \\
\text { to } \\
\text { level. }\end{array}$ & $\begin{array}{l}\text { Ref. } \\
\text { obs. }\end{array}$ & $21 \mathrm{st}$. & $\begin{array}{c}\text { Red. } \\
\text { to } \\
\text { letel. }\end{array}$ & $\begin{array}{l}\text { Ref. } \\
\text { obs. }\end{array}$ & $22 d$. & $\begin{array}{c}\text { Red. } \\
\text { to } \\
\text { level. }\end{array}$ & $\begin{array}{l}\text { Ref. } \\
\text { ots. }\end{array}$ \\
\hline \multirow[b]{2}{*}{1} & 17.8 & -7.21 & .6 & 1.1 & 12.3 & 3.7 & 16.5 & -6.7 & 9.8 & 165 & -8.2 & 8.3 & & & & & & \\
\hline & & & 9.8 & 1.0 & & .3 & 16.5 & " & 9.8 & 17 & " & 8.8 & 17.3 & -9.6 & 7.7 & 3.5 & +3.2 & 6.7 \\
\hline \multirow[t]{2}{*}{2} & 16.0 & -7.3 & 8.7 & $=0.0$ & $\begin{array}{l}-7.6 \\
-7.5\end{array}$ & 12.5 & $\begin{array}{l}10.0 \\
16.0\end{array}$ & 6: & 4.3 & $\begin{array}{l}16.4 \\
16.8\end{array}$ & -8.3 & $\begin{array}{l}11.1 \\
8.5\end{array}$ & 17.0 & " & 8.0 & $\begin{array}{l}3.9 \\
4.3\end{array}$ & +3.1 & 7.4 \\
\hline & & & & & & & & & & & & & 17.6 & "6 & 8.0 & 4.4 & +3.0 & \\
\hline 3 & 14.9 & -7.4 & 7.5 & 18.0 & $"$ & 10.5 & 15.1 & -6.8 & 8.3 & 160 & "6 & 7.7 & 17.6 & -9.7 & 7.9 & 4.5 & +2.9 & 7.4 \\
\hline 4 & 12.0 & -7.5 & 4.5 & 16.5 & " & 9.0 & 13.8 & "6 & 7.0 & 14.5 & -8.4 & 6.1 & 16.5 & " & 6.8 & $\begin{array}{l}4.0 \\
4.0\end{array}$ & +2.8 & 6.8 \\
\hline 5 & 10.4 & "6 & 2.9 & 14.3 & -7.4 & 6.9 & 11.4 & -6.9 & 4.5 & 12.7 & " & 4.3 & $13.6^{2}$ & "6 & 3.9 & 3.2 & +2.6 & 5.8 \\
\hline \multirow[t]{2}{*}{6} & 9.8 & -7.6 & 2.2 & 13.5 & " & 0.1 & 10.2 & "6 & 3.3 & 12.0 & -8.5 & 3.5 & 13.4 & -9.8 & 3.6 & 3.5 & +2.5 & 6.0 \\
\hline & 9.0 & " & 1.4 & - . & "6 & & 9.5 & -7.0 & 2.5 & & & & 13.2 & $" 6$ & 3.4 & & & \\
\hline \multirow[t]{2}{*}{7} & 9.0 & -7.7 & 1.3 & 7.2 & -7.3 & -0.1 & 9.0 & -7.1 & 1.9 & 11.6 & $" 4$ & 3.1 & 13.11 & "6 & 3.2 & 3.3 & +2.4 & 5.7 \\
\hline & 10.0 & " & 2.3 & 7.5 & " & 0.2 & 10.0 & -7.2 & 2.8 & 11.2 & " & 2.7 & 13.4 & ' & 3.2 & 3.0 & 46 & 5.4 \\
\hline 8 & 11.0 & " & 3.3 & 7.5 & $" 4$ & 11.5 & 10.2 & -7.3 & 2.2 & 11.2 & $-8.6^{2}$ & 2.6 & 13.0 & 6 & 3.2 & 3.1 & +2.3 & 5.3 \\
\hline \multirow[t]{2}{*}{9} & 135 & $\$$ & & 9.6 & " & 2.3 & & 66 & -1 & & "6 & 2.9 & 13. & $"$ & 3.2 & 2.8 & " & 5.1 \\
\hline & & & & & -1.2 & 4.8 & 12.0 & & & $1<0$ & -8 & 3.61 & & & & 3.0 & +2.2 & 5.2 \\
\hline 10 & 16.1 & -7.8 & 8.3 & 14.5 & " & 7.3 & 14.9 & -7.4 & 7.5 & 14.5 & -8.5 & 5.7 & 16.7 & " & 6.9 & 3.5 & r & 5.7 \\
\hline \multirow[t]{2}{*}{11} & 19.3 & " & $11.5^{\prime}$ & 17.0 & -7.1 & 9.9 & 17.3 & "6 & 9.9 & 17.5 & $-\$ .9$ & 8.6 & 19.1 & " & 9.3 & 4.3 & "6 & 6.5 \\
\hline & 20.0 & "6 & $\begin{array}{l}12.2 \\
13.0\end{array}$ & 15.5 & 70 & 115 & 109 & & 7 & & 0 & & 10.5 & & 0. & 5.8 & , & 77 \\
\hline Noon & 20.8 & "6 & 13.0 & 19.0 & -16 & 12.0 & $\begin{array}{l}19.2 \\
19.5\end{array}$ & -1.5 & $\begin{array}{l}11.0 \\
12.0\end{array}$ & $\begin{array}{l}19.5 \\
20.0\end{array}$ & -9.0 & 11.0 & 19.2 & & 9.6 & 0.6 & +2 & 1.1 \\
\hline \multirow[t]{2}{*}{1} & 20.13 & " & 12.8 & 19.0 & -6.9 & 12.1 & 20.0 & $" 6$ & 12.5 & 20.5 & -9.1 & 11.4 & 20.0 & "6 & 10.1 & 7.2 & +2.0 & 9.2 \\
\hline & 20.5 & $"$ & 12.7 & 18.9 & " & 12.0 & 20.5 & -7 & 13.0 & 20.5 & " & 11.4 & & & & & & \\
\hline 2 & 19.0 & -7.9 & 11.1 & 18.5 & -6.5 & 11.7 & {$\left[\begin{array}{ll}20.0 \\
19.6\end{array}\right.$} & -7.6 & 12.4 & 201.6 & " & 11.5 & 1.0 & $\begin{array}{r}+9.6 \\
\end{array}$ & 10.6 & 8.0 & +1.8 & 9.8 \\
\hline \multirow[t]{2}{*}{3} & 17.0 & " & 9.1 & 16.3 & " & 0.5 & 19.0 & “ & 11.4 & $\begin{array}{l}20.0 \\
20.2\end{array}$ & "6 & 11.0 & 5.2 & +4.9 & 10.1 & S.4 & +1.6 & 10.0 \\
\hline & & & & & & & & & & & & & 5.2 & & 10.1 & 8.5 & & 10.1 \\
\hline 4 & 16.0 & " & 8.1 & 14.4 & " & 7.6 & 17.4 & -7.7 & 9.7 & 15.9 & -9.3 & 9.6 & 5.0 & +4.8 & 2.8 & 8.5 & +1.4 & 9.9 \\
\hline \multirow[t]{2}{*}{5} & 12.0 & " & 4.1 & 11.0 & " & 4.2 & 15.4 & “ & 7.7 & $1 \div .0$ & " & 7.7 & $\begin{array}{l}4.3 \\
3.9\end{array}$ & +4 & 8.5 & 8.5 & +1 & 9.8 \\
\hline & 11.4 & 6 & 3.5 & & & & & & & & & & & & & 8.0 & & 9.3 \\
\hline 6 & 11.0 & " & 3.1 & 10.0 & " & 3.2 & 13.0 & -7.8 & 5.2 & 15.2 & -9.4 & 5.5 & 3.2 & +4.4 & 7.6 & 4.8 & +1.2 & 6.0 \\
\hline \multirow[t]{2}{*}{7} & 11.0 & " & 3.1 & 9.0 & " & 2.2 & 12.0 & -7.9 & 4.1 & 13.5 & " & 4.1 & 1.6 & +42 & 5.8 & 5.6 & +1.1 & 0.7 \\
\hline & 11.4 & "6 & 3.5 & 8.7 & $" 6$ & 1.9 & 11.0 & & 3.1 & & & & 1.0 & & & & & \\
\hline \multirow[t]{2}{*}{8} & 12.0 & " & 4.1 & 8.0 & 16 & 1.2 & 10.3 & -8.0 & 2.3 & 13.2 & -9.5 & 3.7 & 0.3 & +4.0 & 4.3 & 4.6 & +1.0 & 5.6 \\
\hline & & & & 10.3 & 16 & 3.5 & 11.0 & 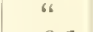 & 3.0 & 13.1 & $"$ & 3.6 & 0.4 & & 4.4 & & & \\
\hline 9 & 14.5 & "6 & 0.6 & 11.1 & -6.7 & 4.4 & 12.5 & -8.1 & 4.4 & 13.1 & $"$ & 3.6 & 0.6 & +3.8 & $\begin{array}{r}4.4 \\
4.2\end{array}$ & 3.1 & +0.9 & 4.0 \\
\hline \multirow[t]{2}{*}{10} & 17.5 & 16 & 9.6 & 14.0 & "6 & 7.3 & 14.5 & "6 & 6.4 & 13.7 & -9.6 & 4.1 & 0.8 & +3.6 & 1.1 & 3.1 & +0.8 & 3.9 \\
\hline & & & & & & & & & & & & & 0.5 & & 4.1 & 3.1 & +0.7 & 3.8 \\
\hline \multirow[t]{2}{*}{$1]$} & 19.7 & -7.8 & 11.9 & 16.2 & "6 & 9.5 & 15.9 & " & 7.8 & 14.7 & "6 & 5.1 & 0.2 & +3.4 & 3.6 & 3.1 & +0.6 & 3.7 \\
\hline & {$[2(1.3$} & "؛ & 13.5 & & & & & & & & & & 1.2 & & 4.6 & 3.5 & +0.5 & 4.0 \\
\hline Mnth $t$ & 1.7 & 2 & 13.6 & & $"$ & 9.8 & 16.1 & -0.2 & 7.9 & 16.2 & "6 & 6.6 & 1.7 & +3.3 & 5.0 & 4.1 & +0.4 & 4.5 \\
\hline
\end{tabular}

Nov. 17. The scale reals up to :20, Jwence the realing 1.4 at mirlnight is equivalent to 21.4.

Nov. 21. At 1 P. H. tha upwer linit of tho scale was reacled, the index was changed afterwards. 
Sertes I.-Tida Onservations from Octorer 10, 1853, to Deceniber 28, 1853.

IIourly observations on the pulley-gauge. Adopted reading of mean level 7.0, expressed in nnits of the seale. Inereasing numbers indicate rise of water.

\section{November, 1853.}

\begin{tabular}{|c|c|c|c|c|c|c|c|c|c|c|c|c|c|c|c|c|c|c|}
\hline $\begin{array}{l}\text { Mean } \\
\text { solar } \\
\text { hour. }\end{array}$ & $23 d$. & $\begin{array}{c}\text { Red. } \\
\text { to } \\
\text { level. }\end{array}$ & $\begin{array}{l}\text { Ref. } \\
\text { obs. }\end{array}$ & $24 t h$. & $\begin{array}{c}\text { Red. } \\
\text { to } \\
\text { level. }\end{array}$ & $\begin{array}{l}\text { Ref. } \\
\text { obs. }\end{array}$ & 25 h. & $\begin{array}{c}\text { Resl. } \\
\text { to } \\
\text { Jevel. }\end{array}$ & $\begin{array}{l}\text { Ref. } \\
\text { abs. }\end{array}$ & 26 th. & $\begin{array}{c}\text { Red. } \\
\text { to } \\
\text { level. }\end{array}$ & $\begin{array}{l}\text { Ref. } \\
\text { obs }\end{array}$ & 27 th. & $\begin{array}{c}\text { Red. } \\
\text { to } \\
\text { letel. }\end{array}$ & $\begin{array}{l}\text { Ref. } \\
\text { obs. }\end{array}$ & 28 th. & $\begin{array}{c}\text { Red, } \\
\text { to } \\
\text { level. }\end{array}$ & $\begin{array}{l}\text { Ref. } \\
\text { obs. }\end{array}$ \\
\hline \multirow[b]{2}{*}{1} & \multirow[b]{2}{*}{4.7} & \multirow[b]{2}{*}{+0.4} & \multirow[b]{2}{*}{5.1} & 6.7 & -3.0 & 3.7 & 10.0 & -6.3 & 3.7 & 14.8 & -10.3 & 4.5 & \multirow[b]{2}{*}{14.7} & \multirow[b]{2}{*}{-10.4} & \multirow[b]{2}{*}{4.3} & \multirow[b]{2}{*}{17.5} & \multirow{2}{*}{\multicolumn{2}{|c|}{5.5}} \\
\hline & & & & 7.4 & -3.2 & 4.2 & 10.2 & $" 6$ & 3.9 & 14.5 & -10. & 4.2 & & & & & & \\
\hline \multirow[t]{2}{*}{2} & \multirow[t]{2}{*}{5.6} & \multirow{2}{*}{+0.2} & \multirow{2}{*}{5.8} & \multirow{2}{*}{8.1} & \multirow{2}{*}{-3.0} & \multirow[t]{2}{*}{$\begin{array}{l}4.5 \\
4.7\end{array}$} & \multirow{2}{*}{10.7} & \multirow{2}{*}{-6.5} & \multirow{2}{*}{$\begin{array}{l}4.11 \\
4.2\end{array}$} & 14.7 & -10.4 & $\begin{array}{l}4.0 \\
4.3\end{array}$ & 13.7 & "6 & 3.3 & 15.3 & -12.1 & 3.2 \\
\hline & & & & & & & & & & & & & 13.3 & 16 & 2.9 & 14.9 & & 2.8 \\
\hline 3 & 6.2 & 0.0 & 6.2 & 9.1 & -3.5 & 5.6 & 11.2 & -6.6 & 4.6 & 15.2 & -10.5 & 4.7 & 13.1 & -10.3 & 2.8 & 14.7 & -12.2 & 2.5 \\
\hline 4 & 7.0 & -0.2 & 6.8 & 10.0 & -3.6 & 6.4 & 12.5 & -6.7 & 5.8 & 15.5 & -10.6 & 4.9 & $\begin{array}{l}13.0 \\
13.2\end{array}$ & -10.4 & $\begin{array}{l}2.6 \\
2.8\end{array}$ & $\mid \begin{array}{l}14.5 \\
14.5\end{array}$ & -123 & $\begin{array}{l}2.3 \\
2.2\end{array}$ \\
\hline & & & & & & & & & & & & & & & & 14.8 & & 2.5 \\
\hline 5 & 7.0 & -0.3 & 6.7 & 10.5 & -3.7 & 6.8 & 13.6 & -6.9 & 6.7 & 16.5 & " & 5.9 & 16.0 & " & 5.6 & 15.6 & -12.4 & 3.2 \\
\hline 6 & 7.5 & -0.5 & 7.0 & 11.1 & -3.8 & 7.3 & 15.0 & -7.0 & 8.0 & 18.0 & -10.7 & 7.3 & 17.3 & " & 6.9 & 18.2 & -12.5 & 5.7 \\
\hline & 7.5 & -0.6 & 6.9 & & & & 15.6 & & 8.6 & & & & & & & & & \\
\hline 7 & 7.6 & -0.7 & 6.9 & 11.6 & -3.9 & 7.7 & 15.7 & -7.1 & 8.6 & 19.4 & " & 8.7 & 19.8 & -10.5 & 9.3 & 20.3 & -12.6 & 7.7 \\
\hline & 7.8 & -0.8 & 7.0 & 11.9 & $" 6$ & 8.0 & 16.0 & -7.2 & 8.8 & & & & & & & & & \\
\hline 8 & 7.9 & -0.9 & 7.0 & 12.5 & -4.0 & 8.5 & 15.6 & -7.3 & 8.3 & 20.0 & -10.8 & 9.2 & 20.9 & -10.6 & 10.3 & 22.6 & -12.7 & 9.9 \\
\hline & 7.8 & -0.9 & 6.9 & 11.6 & -4.1 & 7.5 & & & & 20.2 & & 9.4 & 21.2 & & 10.6 & 23.8 & " & 11.1 \\
\hline 9 & 7.7 & -1.0 & 6.7 & 11.0 & -4.2 & 6.8 & 15.2 & -7.4 & 7.8 & 20.0 & s6 & 9.2 & 21.5 & -10.7 & 10.8 & 24.5 & -12.8 & 11.7 \\
\hline & 7.7 & "6 & 6.7 & & & & & & & 19.5 & c & 8.7 & 21.6 & " & 10.9 & 24.7 & 6 & 11.9 \\
\hline 10 & 7.7 & -1.1 & 6.6 & 9.5 & -4.4 & 5.1 & 15.7 & -7.6 & 8.1 & 19.0 & -10.7 & 8.3 & 21.3 & -10.8 & 10.5 & 24.8 & $"$ & 12.0 \\
\hline & 7.7 & “ & 6.6 & & & & & & & & & & 20.9 & & 10.1 & 24.3 & 6 & 11.5 \\
\hline 11 & 80 & -1.2 & 6.8 & $\begin{array}{l}9.0 \\
8.8\end{array}$ & -4.5 & $\begin{array}{l}4.5 \\
4.3\end{array}$ & 16.0 & -7.8 & 8.2 & 17.8 & "6 & 7.1 & 20.9 & -10.9 & 10.0 & 23.8 & -12.9 & 10.9 \\
\hline Noon & 8.6 & -1.3 & 7.3 & 8.0 & -4.6 & 3.4 & 15.5 & -8.0 & 7.5 & 16.3 & "6 & 5.6 & 19.2 & "6 & 8.3 & 22.7 & " & 9.8 \\
\hline & & & & 8.9 & & 4.3 & 15.5 & -8.1 & 7.4 & & & & & & & & & \\
\hline 1 & 9.6 & -1.5 & 8.1 & 9.9 & -4.7 & 5.2 & 15.4 & -8.2 & 7.2 & 15.7 & " & 5.0 & 18.2 & -11.0 & 7.2 & 21.4 & -13.0 & 8.4 \\
\hline 2 & 10.4 & -1.6 & 8.8 & 10.3 & -4.8 & 5.5 & $\begin{array}{l}15.2 \\
15.8\end{array}$ & $\begin{array}{l}-5.3 \\
-8.4\end{array}$ & $\begin{array}{l}6.9 \\
7.4\end{array}$ & $\begin{array}{l}15.4 \\
15.1\end{array}$ & "6 & $\begin{array}{l}4.4 \\
4.4\end{array}$ & 17.1 & "6 & 6.1 & 18.4 & " & 5.4 \\
\hline 3 & & & & & & & & & & 15.0 & & 4.3 & & & & & & \\
\hline 3 & 11.4 & -1.8 & 9.6 & 11.4 & -4.9 & 6.5 & 16.0 & -8.6 & 7.4 & $\begin{array}{l}15.1 \\
15.4\end{array}$ & $" 6$ & $\begin{array}{l}4.4 \\
4.7\end{array}$ & $\begin{array}{l}15.2 \\
14.9\end{array}$ & -11. & $\begin{array}{l}4.1 \\
3.8\end{array}$ & $\begin{array}{l}17.1 \\
15.5\end{array}$ & “ & $\begin{array}{l}4.1 \\
2.8\end{array}$ \\
\hline 4 & 11.8 & -2.0 & 9.8 & 12.6 & -5.0 & 7.6 & 16.4 & -8.8 & 7.6 & 15.5 & -10.6 & 4.9 & 14.6 & -11.2 & 3.4 & 15.7 & " & 2.7 \\
\hline & 12.0 & -2.1 & 9.9 & & & & & & & & & & 16.9 & & 5.7 & 15.6 & " & 2.6 \\
\hline 5 & 12.0 & -2.2 & 9.8 & 13.5 & -5.1 & 8.4 & 18.1 & -9.0 & 9.1 & 17.0 & "c & 6.4 & 17.5 & -11.3 & 6.2 & 15.9 & 64 & 2.9 \\
\hline & 12.0 & -2.3 & 9.7 & 13.9 & -5.2 & 8.7 & 19.1 & -9.1 & 10.0 & & & & & & & 16.4 & 46 & 3.4 \\
\hline 6 & 12.2 & $" 6$ & 9.9 & $14.6^{\circ}$ & -5.3 & 9.3 & 19.6 & -9.3 & 10.3 & 19.1 & " & 8.5 & 19.3 & -11.4 & 7.9 & 17.7 & " 6 & 4.7 \\
\hline & 11.8 & -2.4 & 9.4 & 14.2 & -5.4 & 9.5 & 19.7 & -9.4 & 10.3 & & & & & & & & & \\
\hline 7 & 11.4 & "6 & 9.0 & 14.4 & -5.5 & 8.9 & 19.8 & -9.5 & 10.3 & 20.0 & " & 9.4 & 20.9 & -1 & 9.4 & 20.0 & " & 7.0 \\
\hline & & & & 14.4 & -5.6 & 8.8 & 20. & -9.7 & 10.3 & 20.5 & & 9.9 & 22.0 & & 10.5 & & & \\
\hline 8 & 10.0 & -2.5 & 7.5 & 13.7 & -5.7 & 8.0 & 19.4 & -9.8 & 9.6 & 20.4 & -10.5 & 9.9 & 22.1 & -11.6 & 10.5 & 22.0 & " & 9.0 \\
\hline 9 & 90 & -26 & 6.4 & 128 & -5.9 & 6.9 & 78.5 & -9.9 & 8.6 & 20.4 & a & $\begin{array}{l}3.9 \\
9.9\end{array}$ & 22.0 & -11.7 & 11.2 & 23.7 & "6 & 10.7 \\
\hline & & & & & & & & & & 20.7 & & 96 & 22.9 & & 11.2 & 23. & ؛ & 09 \\
\hline 10 & 8.2 & -2.7 & 5.5 & 12.5 & -6.0 & 6.5 & 18.0 & -10.0 & 8.0 & $19 . S$ & " & 9.3 & 22.6 & -11.5 & 10.8 & 23.9 & $" 6$ & 10.9 \\
\hline & & & & & & & & & & & & & 22.1 & & 10.3 & 24.0 & & 10 \\
\hline 11 & 7.7 & -2.8 & 4.9 & 11.5 & -6.1 & 5.4 & 16.0 & -10.1 & 5.9 & 17.7 & " & 7.2 & 21.6 & -11.9 & 9.7 & 23.5 & 6 & 12.5 \\
\hline lidn't & 7.4 & -2.9 & 4.5 & 109 & -62 & 4. & 15.0 & 103 & 4.7 & 162 & -10.4 & 5.8 & 19.7 & -790 & 7.7 & $\begin{array}{l}21.1 \\
20.8\end{array}$ & " "6 & $\begin{array}{l}8.1 \\
7.8\end{array}$ \\
\hline Holian't & 1.2 & -3.0 & 4.2 & & & 4.1 & & & 4.8 & & & & & -12.0 & & & & \\
\hline
\end{tabular}

Nov, 26. From 11 A. M. of this day two readings are given for each half honr; the mean of the two observations has been inserted above. The two corresponding readiugs agree generally within a fer tenths, the difference being due to the effect of the small waves.

Nov. 28. Orders were given to observe and record careful soundings by lead line at the tide hole every day at twelve o'clock. 
Series I.-Tidal Observations from October 10, 1 S53, to December 28, 1 s53.

Hourly observatious on the pulley-gauge. Adopted reading of mean level 7.0, expressed iu units of the scale. Increasing numbers indieate rise of water.

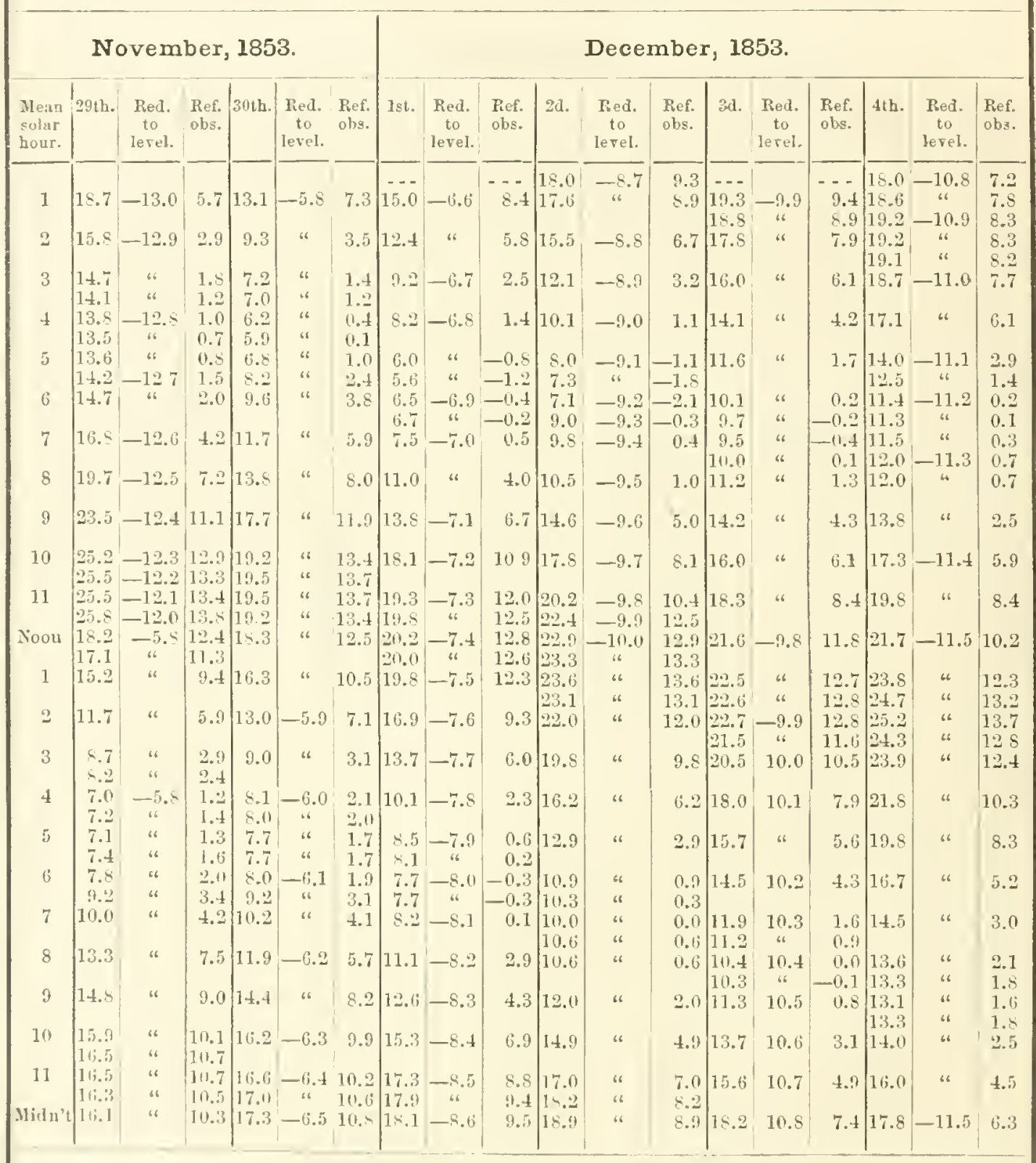

Nov. 29. Tide registrr correcterl at moon. Sounding at noon 7 fath., 0 feet, 4 inclues, register 18.2 . 1t may be remarked that sounlings are sulject to uncertainly in easo of auy drift of the ice field in which the vessel was

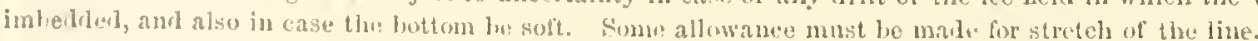

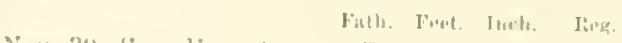

\begin{tabular}{|c|c|c|c|c|c|c|c|c|}
\hline Nov. & 311. & Sonumling & at noon & 7 & 2 & $: 3$ & 10.2 & ('This soumling was not userl, apparently not lecliable.) \\
\hline 116.: & 1. & 6 & a & 8 & 11 & 4 & 20.2 & \\
\hline “ & 2. & “ & " & 7 & 5 & 6 & 32.7 & A nean correction was user for thess: lays, as denluced \\
\hline " & 3. & "6 & " & & 11 & 11 & 22.11 & from enveloping curves and the soundings. \\
\hline 61 & 4. & $" 6$ & "6 & & . I & 0 & & \\
\hline
\end{tabular}


Series 1.-Tidal Onservations frou Oćtober 10, 1853, to Dechamer 28, 1853.

Hourly observations on the pulley-gauge. Adopted reading of mean level 7.0, expressed in units of the seale. Increasing numbers indicate rise of water.

\section{December, 1853.}

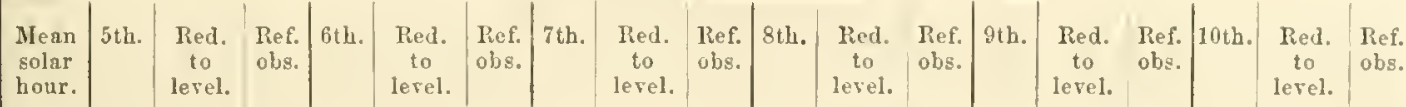

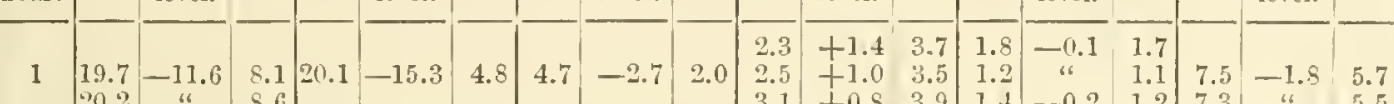

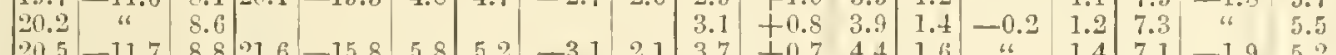

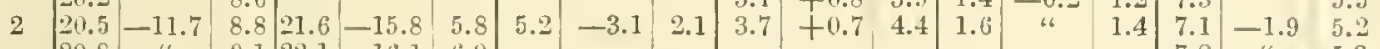

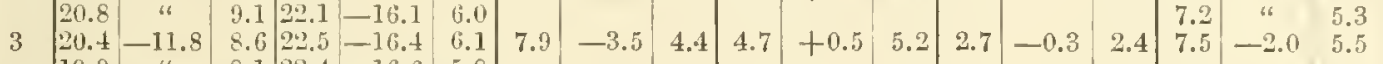

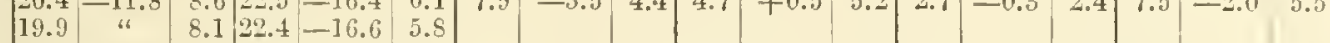

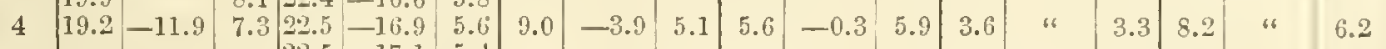

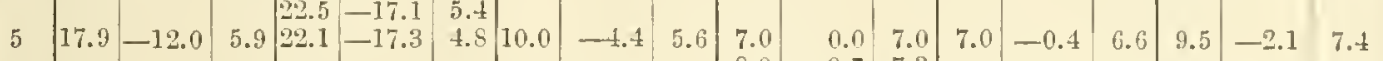

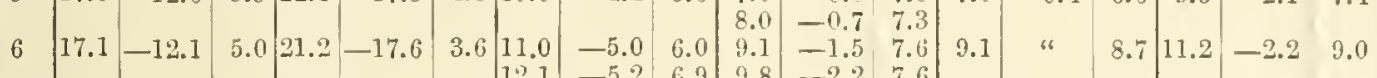

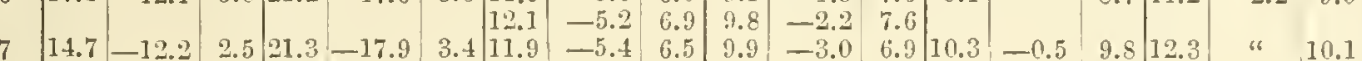

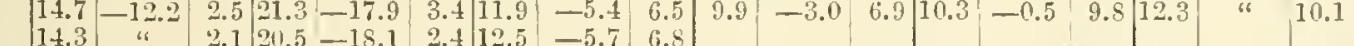

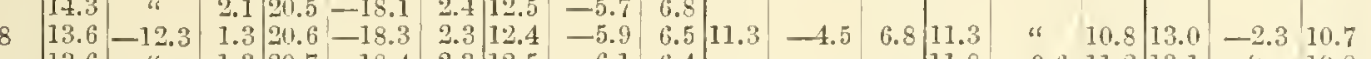

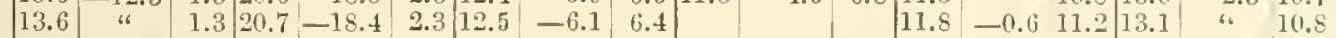

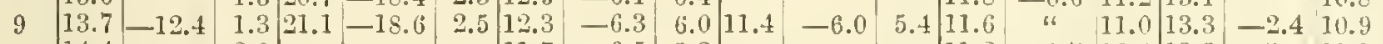

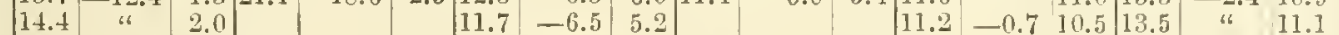

\begin{tabular}{l|l|l|l|l|l|l|l|l|l|l|l|l|l|l|l|l|l|l|l|l}
10 & 15.2 & -12.5 & 2.7 & 21.6 & -18.9 & 2.7 & 12.2 & -6.5 & 5.4 & 12.4 & -7.5 & 4.9 & 10.7 & -0.7 & 10.5 & 13.5 & -2.5 & 10.3 & -2.5 & 10.8
\end{tabular}

\begin{tabular}{l|l|l|l|l|l|l|l|l|l|l|l|l|l|l|l|l|l|l}
11 & 17.5 & -12.6 & 5.2 & 22.7 & -19.3 & 3.4 & 11.2 & -7.3 & 3.9 & 13.2 & -9.0 & 4.2 & 9.7 & -0.8 & 8.9 & 11.8 & " & 9.3
\end{tabular}

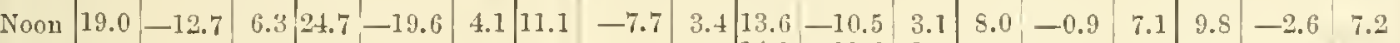

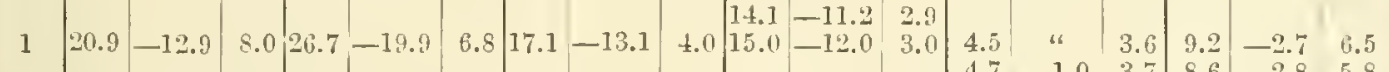

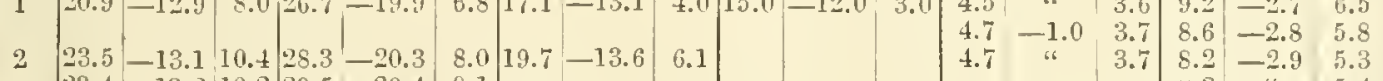

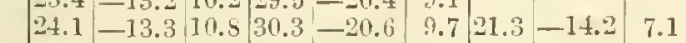

$24.3-13.211 .1 \mid 31.0-20.810 .2$

$4 \quad 24.1-13.510 .631 .3-21.0 \quad 10.324 .2-14.7 \quad 9.5$

\begin{tabular}{lll}
0 & $31.5-21.210 .3$ \\
\hline
\end{tabular}

$23.2-13.7 \quad 9.531 .5-21.310 .24 .9+4.89 .7$

\begin{tabular}{l|l|l|l|l|} 
& & 30.5 & -21.5 & 9.0
\end{tabular}

$21.6-13.9 \quad 7.7 .5 .5-21.70 .85 .3+4.40 .7$

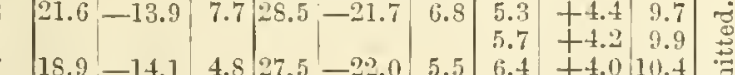

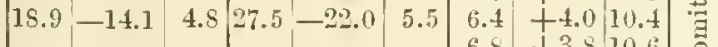

$17.2-14.23 .024 .7-22.4 \quad 2.3 \quad 7.0+3.610 .6$ क

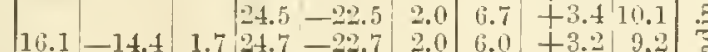

$15.5-14.5 \quad 1.7 \mid 24.7-22.7 \quad 2.0$

$4.8-14.6$ - 1.2 .76 .9

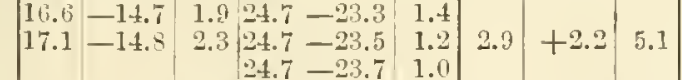

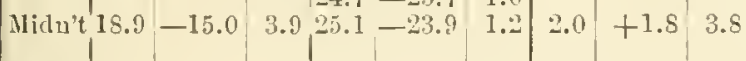

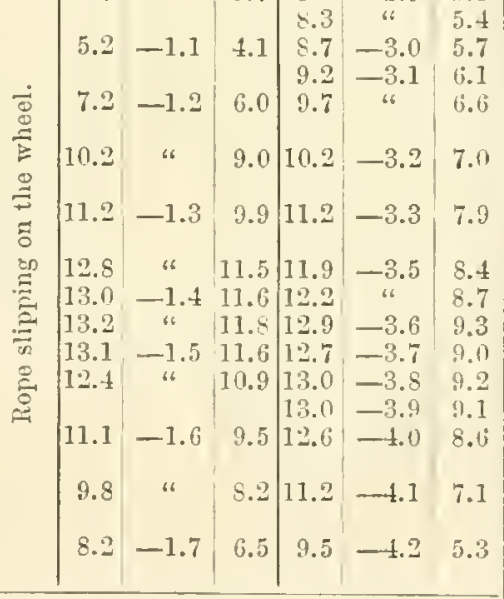

Fath. Feet. Inch, reg.

Dec.5. Sonnding at noon $7 \quad 10$ 20, cliangel to 19. ) On these days the corrections dednced

"6. "6 "

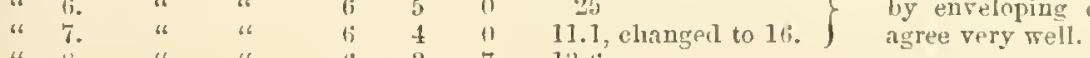

"8.

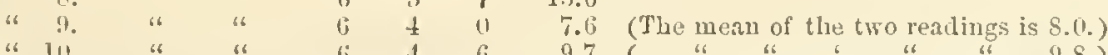

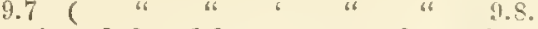

From the 9 th to the 1 sth of December the corrections deduced from curres and soundings differ by a constant of nearly 4 feet. The differences are partly due to imperfect soumlings, partly to snddeu changes of the pulleygange (see readings between noon and 1 P. $\mathbf{M}$. on the (th). The heights giren, as corrected by the soundings aud curves, are, during this peliod, of little value, the times being less affected. The soundings were increastel by 4 feet, equal to a reading of the mean level of 32.6 . 
Series I.-Tidal Oberrvations fron October 10, 1853, to Deceniber 28, 1853.

Hourly observations on the pulley-gauge. Adopted reading of mean level 7.0, expressed in units of the seale. Increasing numbers indicate rise of water.

December, 1853.

\begin{tabular}{|c|c|c|c|c|c|c|c|c|c|c|c|c|c|c|c|c|c|c|}
\hline $\begin{array}{l}\text { Mean } \\
\text { solar } \\
\text { hour. }\end{array}$ & 11th. & $\begin{array}{c}\text { Red. } \\
\text { to } \\
\text { level. }\end{array}$ & $\begin{array}{l}\text { Rer. } \\
\text { obs. }\end{array}$ & 12 th. & $\begin{array}{c}\text { Red. } \\
\text { to } \\
\text { level. }\end{array}$ & $\begin{array}{l}\text { Ref. } \\
\text { obs. }\end{array}$ & 13th. & $\begin{array}{c}\text { Red. } \\
\text { to } \\
\text { lerel. }\end{array}$ & $\begin{array}{l}\text { Ref. } \\
\text { obs. }\end{array}$ & 14th. & $\begin{array}{c}\text { Red. } \\
\text { to } \\
\text { level. }\end{array}$ & $\begin{array}{l}\text { Ref. } \\
\text { obs. }\end{array}$ & 15 th. & $\begin{array}{c}\text { Red. } \\
\text { to } \\
\text { level. }\end{array}$ & $\begin{array}{l}\text { Ref. } \\
\text { obs. }\end{array}$ & 16 th. & $\begin{array}{c}\text { Red. } \\
\text { to } \\
\text { level. }\end{array}$ & $\begin{array}{l}\text { Ref. } \\
\text { obss. }\end{array}$ \\
\hline 1 & 8.2 & -4.3 & 3.9 & 12.2 & -8.5 & 3.4 & $\begin{array}{l}21.4 \\
20.7\end{array}$ & $\begin{array}{l}-14.0 \\
-14.1\end{array}$ & $\begin{array}{l}7.4 \\
6.6\end{array}$ & \begin{tabular}{l|l} 
\\
6 & 13.9
\end{tabular} & -7.6 & 6.3 & 15.4 & $-\$ .5$ & 6.9 & $\begin{array}{l}23.0 \\
22.7\end{array}$ & $\begin{array}{r}-10.6 \\
-10.8\end{array}$ & $\begin{array}{l}12.4 \\
11.9\end{array}$ \\
\hline 2 & 7.4 & -4.4 & 3.0 & 11.1 & -9.1 & 2.0 & 20.3 & -14.3 & 6.0 & \begin{tabular}{l|l|l|}
0 & 12.9
\end{tabular} & " & 5.3 & 13.7 & " & 5.2 & 20.4 & -11.1 & 9.3 \\
\hline 3 & $\begin{array}{l}6.8 \\
6.3\end{array}$ & $\begin{array}{r}-4.5 \\
-4.6\end{array}$ & $\begin{array}{l}2.3 \\
1.7\end{array}$ & $\left|\begin{array}{l}10.2 \\
10.0\end{array}\right|$ & $\begin{array}{l}-9.3 \\
-9.5\end{array}$ & $\begin{array}{l}0.9 \\
0.5\end{array}$ & $\begin{array}{l}19.8 \\
18.9\end{array}$ & $\begin{array}{l}-14.5 \\
-14.6\end{array}$ & $\begin{array}{l}5.3 \\
4.3\end{array}$ & $\left|\begin{array}{l}11.2 \\
10.2\end{array}\right|$ & -7.7 & $\begin{array}{l}3.5 \\
2.5\end{array}$ & 12.2 & -8.6 & 3.6 & 18.5 & -11.4 & 7.1 \\
\hline 4 & $\begin{array}{l}6.5 \\
7.5\end{array}$ & $\begin{array}{r}-4.7 \\
-4.8\end{array}$ & $\begin{array}{l}1.8 \\
2.7\end{array}$ & $\begin{array}{l}10.0 \\
10.2\end{array}$ & $\begin{array}{r}-9.7 \\
-9.8\end{array}$ & $\begin{array}{l}0.3 \\
0.4\end{array}$ & $\begin{array}{l}18.2 \\
18.7\end{array}$ & $\begin{array}{l}-14.7 \\
-14.8\end{array}$ & $\begin{array}{l}3.5 \\
3.9\end{array}$ & \begin{tabular}{|l|l}
5 & 10.0 \\
10.0
\end{tabular} & “ & $\begin{array}{l}2.3 \\
2.3\end{array}$ & $\begin{array}{l}11.5 \\
11.3\end{array}$ & " & $\begin{array}{l}2.9 \\
2.7\end{array}$ & $\begin{array}{l}16.0 \\
15.3\end{array}$ & $\begin{array}{l}-11.7 \\
-11.5\end{array}$ & $\begin{array}{l}4.3 \\
3.5\end{array}$ \\
\hline 5 & 8.7 & -1.9 & 3.8 & 10.4 & -10.0 & 0.4 & 19.4 & -14.9 & 4.5 & $5 \begin{array}{l}10.0 \\
10.2\end{array}$ & "6 & $\begin{array}{l}2.3 \\
2.5\end{array}$ & $\begin{array}{l}11.1 \\
11.4\end{array}$ & -8.7 & $\begin{array}{l}2.4 \\
2.7\end{array}$ & \begin{tabular}{l|}
15.4 \\
15.9
\end{tabular} & $\begin{array}{l}-12.0 \\
-12.2\end{array}$ & $\begin{array}{l}3.4 \\
3.7\end{array}$ \\
\hline 6 & 11.8 & -5.0 & 6.8 & $|12.9|$ & -10.2 & 2.7 & 29.8 & -15.1 & 5.7 & 10.6 & -7.8 & 2.8 & $\begin{array}{l}11.7 \\
12.2\end{array}$ & $\begin{array}{c}" 6 \\
-8.8\end{array}$ & $\begin{array}{l}3.0 \\
3.4\end{array}$ & 16.1 & -12.3 & 3.8 \\
\hline 7 & 12.4 & -5.1 & 7.3 & 15.5 & -10.4 & 5.1 & 22.5 & -15.3 & 7.2 & 12.1 & " & 4.3 & 12.9 & $"$ " & 4.1 & 17.2 & -12.6 & 4.6 \\
\hline 8 & 13.7 & -5.2 & $\begin{array}{l}8.5 \\
8.9\end{array}$ & 18.0 & -10.7 & 7.3 & 24.7 & -15.5 & 9.2 & 15.1 & -7.9 & 7.2 & 15.2 & " & 6.4 & 19.1 & -12.9 & 6.2 \\
\hline 9 & $\begin{array}{l}14.7 \\
15.0\end{array}$ & $\begin{array}{l}-5.4 \\
-5.5\end{array}$ & $\begin{array}{l}9.3 \\
9.5\end{array}$ & 19.7 & -10.9 & 8.8 & 25.7 & -15.7 & 10.0 & 0 & “ & 9.3 & 17.8 & -8.9 & 8.9 & 21.2 & -13.4 & 7.8 \\
\hline 10 & 15.0 & $\begin{array}{r}\text { " } \\
-5.6\end{array}$ & $\begin{array}{l}9.5 \\
9.1\end{array}$ & 20.4 & $\begin{array}{l}-11.1 \\
-11.2\end{array}$ & $\begin{array}{l}9.3 \\
9.6\end{array}$ & $\left|\begin{array}{l}26.7 \\
27.0\end{array}\right|$ & -15.9 & 10.8 & $8 \mid \begin{array}{ll}19.4 \\
20.2\end{array}$ & -8.0 & 11.4 & 19.6 & " & 10.7 & 24.0 & -13.7 & 10.3 \\
\hline 11 & $\mid \begin{array}{l}14.8 \\
14.3\end{array}$ & $" .0$ & 8.7 & 21.2 & $\begin{array}{l}-11.3 \\
-11.4\end{array}$ & $\begin{array}{l}0.9 \\
8.8\end{array}$ & $\begin{array}{r}27.0 \\
27.1\end{array}$ & $\begin{array}{r}-16.1 \\
-16.2\end{array}$ & $\begin{array}{l}10.9 \\
10.9\end{array}$ & {$\left[\begin{array}{l}20.5 \\
20.2\end{array}\right.$} & " & $\begin{array}{l}12.5 \\
12.2\end{array}$ & 20.5 & " & 11.6 & 26.0 & -14.1 & 11.9 \\
\hline Noon & 13.4 & -5.7 & 7.7 & 20.2 & -11.5 & 8.7 & $\begin{array}{l}26.8 \\
12.0\end{array}$ & $\begin{array}{c}-16.3 \\
?\end{array}$ & 10.5 & 19.6 & -8.1 & 11.5 & 21.0 & -9.0 & $\left|\begin{array}{l}12.0 \\
13.0\end{array}\right|$ & $\left|\begin{array}{l}27.3 \\
27.5\end{array}\right|$ & $\begin{array}{l}-14.5 \\
-14.7\end{array}$ & $\begin{array}{l}12.5 \\
12.8\end{array}$ \\
\hline 1 & 11.2 & -5.9 & 5.3 & 20.2 & -11.7 & 8.5 & 11.9 & & $\ldots$ & -18.2 & " & 10.1 & $\left|\begin{array}{l}22.3 \\
21.2\end{array}\right|$ & -9.1 & $\begin{array}{l}13.2 \\
12.1\end{array}$ & $\begin{array}{l}27.3 \\
26.8\end{array}$ & $\begin{array}{r}-14.8 \\
-15.0\end{array}$ & $\begin{array}{l}12.5 \\
11.5\end{array}$ \\
\hline 2 & $\begin{array}{l}9.4 \\
8.7\end{array}$ & $\begin{array}{l}-6.1 \\
-6.2\end{array}$ & $\begin{array}{l}3.3 \\
2.5\end{array}$ & 18.5 & -11.9 & 6.6 & 10.9 & ? & - . & 16.2 & “ & 8.1 & 20.1 & -9.2 & 10.9 & 26.0 & -15.1 & 10.9 \\
\hline 3 & $\begin{array}{r}8.3 \\
8.3\end{array}$ & $\begin{array}{l}-6.4 \\
-6.5\end{array}$ & $\begin{array}{l}1.9 \\
1.8\end{array}$ & 16.8 & -12.1 & 4.7 & 10.8 & -7.1 & 3.7 & 813.8 & -8.2 & 5.6 & 17.6 & -9.3 & 8.3 & 23.1 & -15.3 & 7.8 \\
\hline 4 & 8.7 & -6.7 & 2.0 & $\left|\begin{array}{l}15.9 \\
15.2\end{array}\right|$ & $\begin{array}{l}-12.3 \\
-12.4\end{array}$ & $\begin{array}{l}3.6 \\
2.5\end{array}$ & $\mid \begin{array}{l}10.2 \\
10.0\end{array}$ & -7.2 & $\begin{array}{l}3.0 \\
2.8\end{array}$ & $\begin{array}{l}11.5 \\
11.1\end{array}$ & " & $\begin{array}{l}3.3 \\
2.9\end{array}$ & 16.2 & -9.4 & 6.8 & $21.0 \mid$ & -15.5 & 5.5 \\
\hline 5 & 9.7 & -6.9 & 2.8 & 15.6 & -12.5 & 3.1 & $\begin{array}{l}10.3 \\
10.3\end{array}$ & " & $\begin{array}{l}3.1 \\
3.1\end{array}$ & $\begin{array}{l}10.8 \\
10.8\end{array}$ & " " & $\begin{array}{l}2.6 \\
2.6\end{array}$ & $\left|\begin{array}{l}15.6 \\
15.4\end{array}\right|$ & -9.5 & $\begin{array}{l}6.1 \\
5.9\end{array}$ & 18.3 & -15.6 & 2.7 \\
\hline 6 & 11.2 & -7.1 & 4.1 & 17.7 & -12.7 & 5.0 & 10.7 & -7.3 & 3.4 & $\begin{array}{l}10.7 \\
10.9\end{array}$ & -8.3 & $\begin{array}{l}2.4 \\
2.6\end{array}$ & $\begin{array}{l}15.1 \\
15.2\end{array}$ & -9.6 & $\begin{array}{l}5.5 \\
5.6\end{array}$ & $\begin{array}{l}17.6 \\
17.5\end{array} \mid$ & -15.5 & $\begin{array}{l}1.8 \\
1.7\end{array}$ \\
\hline 7 & 12.3 & -7.3 & $5.0]$ & $\mid 19.7$ & -12.9 & 6.8 & 12.2 & " & 4.9 & 11.2 & " & 2.9 & 15.7 & -9.7 & 6.0 & $\begin{array}{l}17.5 \\
17.5\end{array} \mid$ & -15.9 & $\begin{array}{l}1.6 \\
1.6\end{array}$ \\
\hline 8 & 13.7 & -7.6 & 6.1 & 21.0 & -13.1 & 7.9 & 13.0 & " & 5.7 & 13.7 & " & 5.4 & 16.8 & -9.8 & 7.0 & 17.7 & -16.0 & 1.7 \\
\hline 9 & $\begin{array}{l}15.2 \\
15.7\end{array}$ & $\begin{array}{l}-7.8 \\
-7.8\end{array}$ & $\begin{array}{l}7.4 \\
7.5\end{array}$ & 21.9 & -13.3 & 8.6 & $\begin{array}{l}15.1 \\
15.7\end{array}$ & -7.4 & $\begin{array}{l}7.7 \\
8.3\end{array}$ & 15.6 & " & 7.3 & 18.8 & -9.9 & 8.9 & $19.7 \mid$ & -16.1 & 3.6 \\
\hline 10 & $\begin{array}{l}15.8 \\
15.8\end{array}$ & $\begin{array}{l}-8.1 \\
-8.3\end{array}$ & $\begin{array}{c}7.7 \\
7.5\end{array}$ & {$\left[\begin{array}{l}22.8 \\
23.2\end{array}\right.$} & $\begin{array}{l}-13.5 \\
-13.6\end{array}$ & $\begin{array}{l}9.3 \\
9.6\end{array}$ & $\begin{array}{l}16.1 \\
16.11\end{array}$ & “ & $\begin{array}{l}8.6 \\
8.6\end{array}$ & $\begin{array}{l}16.9 \\
17.2\end{array}$ & -8.4 & $\begin{array}{l}8.5 \\
8.8\end{array}$ & 21.2 & -10.1 & 11.1 & 21.5 & -16.2 & 5.3 \\
\hline 11 & 15.3 & -8.4 & $6.9:$ & $\begin{array}{l}23.3 \\
23.4\end{array}$ & $\begin{array}{l}-13.7 \\
-13.8\end{array}$ & $\begin{array}{l}9.6 \\
1.6\end{array}$ & $\begin{array}{l}16.0 \\
15.7\end{array}$ & -7.5 & $\begin{array}{l}8.5 \\
8.2\end{array}$ & $\begin{array}{l}17.3 \\
17.2\end{array}$ & " & $\begin{array}{l}8.9 \\
8.5\end{array}$ & $\left|\begin{array}{l}22.4 \\
22.8\end{array}\right|$ & $\begin{array}{l}-10.3 \\
-10.4\end{array}$ & $\begin{array}{l}12.1 \\
12.5\end{array}$ & 23.1 & -16.3 & 5.8 \\
\hline Mirln't & 13.7 & -8.6 & 5.1 & 23.4 & -13.9 & 9.5 & 15.2 & " & 7.7 & 17.1 & " & 8.7 & 23.0 & -10.5 & 12.5 & 23.8 & -16.4 & 7.4 \\
\hline & & & Dec. & . 11. & Soundin & $\mathrm{ng}$ at & noon & $\begin{array}{c}\text { Fath. } \\
6\end{array}$ & $\begin{array}{c}F_{\text {ret. }} \\
2\end{array}$ & $\begin{array}{c}\text { Incit. } \\
9\end{array}$ & $\begin{array}{l}\text { Jifg. } \\
13.3\end{array}$ & & & & & & & \\
\hline & & & “ & 12. & $"$ & " & & 6 & 4 & 0 & 19.9 & & & & & & & \\
\hline & & & " & 13. & " & “" & 6 & 7 & I & 3 & 13.3 & (Chans & aged fr & rom 26.8 & & & & \\
\hline & & & " & 14. & “ & “ & & 7 & 2 & 2 & 19.4 & & & & & & & \\
\hline & & & $"$ & 15. & " & " & & 7 & .4 & $" 1$ & 21.2 & & & & & & & \\
\hline & & & " & 16. & " & " & & 7 & 3 & ij & 27.6 & & & & & & & \\
\hline
\end{tabular}


Series I.-Tidal Onservations from October 10, 1853, to I) ecenber 28, 1853.

IIourly observations on the pulley-gauge. Adopted reading of mean level 7.0, expressed in units of the seale. Inereasing numbers indieate rise of water.

December, 1853.

\begin{tabular}{|c|c|c|c|c|c|c|c|c|c|c|c|c|c|c|c|c|c|c|}
\hline $\begin{array}{l}\text { Mean } \\
\text { solar } \\
\text { hour. }\end{array}$ & 17 th. & $\begin{array}{c}\text { Red. } \\
\text { to } \\
\text { level. }\end{array}$ & $\begin{array}{l}\text { Ref. } \\
\text { obs. }\end{array}$ & 18th. & $\begin{array}{c}\text { Ried. } \\
\text { to } \\
\text { level. }\end{array}$ & $\begin{array}{c}\text { Ref. } \\
\text { obs. }\end{array}$ & I9th. & $\begin{array}{c}\text { Red. } \\
\text { to } \\
\text { level. }\end{array}$ & $\begin{array}{l}\text { Ref. } \\
\text { obs. }\end{array}$ & $20 \mathrm{th}$. & $\begin{array}{c}\text { Red. } \\
\text { to } \\
\text { lerel }\end{array}$ & $\begin{array}{l}\text { Ref. } \\
\text { obs. }\end{array}$ & 21 st. & $\begin{array}{c}\text { Tied. } \\
\text { to } \\
\text { level. }\end{array}$ & $\begin{array}{l}\text { Ref. } \\
\text { obs. }\end{array}$ & $22 d$. & $\begin{array}{l}\text { Red. } \\
\text { to } \\
\text { level. }\end{array}$ & $\begin{array}{l}\text { Ref. } \\
\text { obs. }\end{array}$ \\
\hline \multirow[b]{2}{*}{1} & 24.0 & -16.5 & 7.5 & 23.4 & -17.6 & 5.8 & 4.1 & 1) & 7.1 & & & & & & & & & \\
\hline & 23.7 & & 7.1 & 23.4 & "6 & $\begin{array}{l}5.8 \\
5.8\end{array}$ & 4.2 & $\begin{array}{r}+2.9 \\
+27\end{array}$ & 7.1 & 14.5 & -3.9 & 10.6 & 20.4 & -10.7 & 9.7 & 20.6 & -13.7 & 6.9 \\
\hline \multirow[t]{2}{*}{2} & 23.8 & -16.7 & 7.1 & 23.2 & $" 6$ & 5.6 & 5.7 & $\begin{array}{r}+2.5 \\
+2\end{array}$ & 8.2 & 15.1 & -4.2 & 10.9 & 21.4 & -11.0 & 10.4 & 21.6 & $" 6$ & 7.9 \\
\hline & & & & 22.6 & 6 & 5.0 & 5.7 & +2.3 & 8.0 & 15.3 & -4.4 & 10.9 & 22.4 & -11.1 & 11.3 & & & \\
\hline \multirow[t]{2}{*}{3} & 22.9 & " & 6.2 & 21.7 & " & 4.1 & 5.0 & +2.2 & 7.2 & 15.2 & -4.5 & 10.7 & 22.9 & -11.3 & 11.6 & 22.2 & $"$ & 8.5 \\
\hline & & & & & & & & & & 14.2 & -4.7 & 9.5 & 22.3 & -11.4 & 10.9 & $2-23.4$ & $"$ & 8.7 \\
\hline 4 & 21.5 & -16.8 & 4.7 & 20.2 & " & 2. 6 & 3.7 & +2.0 & 5.7 & 13.5 & -4.8 & 8.7 & 21.3 & -11.6 & 9.7 & $\mid \begin{array}{ll}2 * 2.5 \\
0.0 & 0\end{array}$ & -13.8 & 8.7 \\
\hline 5 & $\begin{array}{l}20.5 \\
19.9\end{array}$ & $"$ " & $\begin{array}{l}3.7 \\
3.7\end{array}$ & 21.3 & " & 3.7 & 2.1 & +1.8 & 3.9 & 12.2 & -5.2 & 7.0 & 21.0 & -11.9 & 9.1 & 22.3 & " & 8.5 \\
\hline \multirow[t]{2}{*}{6} & 19.6 & -16.9 & 2.7 & 20.0 & $"$ & 2.4 & 1.4 & +1.6 & 3.0 & 10.5 & -5.5 & 5.0 & 19.3 & -12.2 & 7.1 & $21 . \pi$ & $"$ & 7.9 \\
\hline & $\mid 19.7$ & & 2.8 & 19.3 & " & 1.7 & 1.1 & +1.4 & 2.5 & & & & & & & & & \\
\hline \multirow[t]{2}{*}{7} & 20.2 & 6 & 3.3 & 18.5 & $"$ & 0.9 & 1.1 & +1.3 & 2.4 & 10.1 & -5.8 & 4.3 & 18.3 & -12.4 & 6.9 & 211.9 & " & 7.1 \\
\hline & & & & 20.2 & " & 2.6 & 0.9 & +1.1 & 2.0 & & & & & & & & & \\
\hline 8 & 21.7 & -17.0 & 4.7 & 20.4 & -17.6 & 2.8 & 1.6 & +1.0 & 2.6 & 9.6 & -6.1 & 3.5 & 17.8 & -12.6 & 5.2 & 19.3 & -13.9 & 4 \\
\hline \multirow[t]{2}{*}{9} & 25.0 & " & 8.0 & 4.3 & ? & $\ldots$ & 2.9 & +0.8 & 3.7 & 8.0 & $\begin{array}{l}-6.3 \\
-6.4\end{array}$ & $\begin{array}{l}1.4 \\
1.6\end{array}$ & 17.4 & $-1:$ & $\begin{array}{l}4.5 \\
4.6\end{array}$ & $\begin{array}{l}19.4 \\
19.0\end{array}$ & " & 5.1 \\
\hline & & & & & & & & & & 8.7 & -6.5 & 2.2 & 17.8 & -12.9 & 4.9 & 19.0 & & 5. \\
\hline 10 & 27.7 & -17.1 & 10.6 & 7.5 & 6 & $\ldots$ & 5.0 & +0.6 & 5.6 & 9.5 & -6.7 & 2.8 & 18.3 & -13.0 & 5.3 & 19.3 & "6 & 5.4 \\
\hline 11 & $\begin{array}{l}2.8 .5 \\
29.7\end{array}$ & $\begin{array}{c}u \\
-17.2\end{array}$ & $\begin{array}{l}11.7 \\
12.5\end{array}$ & 8.3 & $"$ & $\cdots$ & 7.1 & +0.3 & 7.4 & 11.3 & -7.1 & 4.2 & 19.5 & -13.2 & 6.3 & 20.3 & " & \\
\hline Noon & 23.0 & $?$ & -- & 10.9 & $"$ & $\cdots$ & 8.2 & 0.0 & 8.2 & 13.1 & -7.5 & 5.6 & 21.2 & -13.4 & 7.8 & 21.7 & -14.0 & 7.7 \\
\hline \multirow[t]{2}{*}{1} & 22.6 & 6 & $\cdots$ & 12.4 & " & --- & 10.4 & -0.2 & 10.2 & 16.9 & -7.7 & 9.2 & $\begin{array}{l}27.8 \\
27.7\end{array}$ & $?$ & $\begin{array}{l}-.- \\
\cdots-\end{array}$ & 23.0 & " & 9.1 \\
\hline & & & & 12.5 & " & $\ldots$ & 10.7 & -0.3 & 10.4 & & & & & & & & & \\
\hline 2 & 20.0 & "s & $\cdots$ & 12.5 & " & $\cdots$ & 10.7 & -0.5 & 10.2 & 18.2 & -7.9 & 10.3 & 24.4 & -13.4 & 11.0 & 24.5 & -14.1 & 10.4 \\
\hline \multirow{2}{*}{3} & 19.6 & "6 & & $-n$ & & & 10.7 & -0.6 & 10.1 & 18.4 & $\begin{array}{l}-8.0 \\
-8.1\end{array}$ & $\begin{array}{l}11.4 \\
70.4\end{array}$ & $\log _{7}$ & & & $25=3$ & & \\
\hline & & & -- & & & $\cdots$ & 10.0 & -0.8 & 9.8 & $\begin{array}{l}18.2 \\
18.5\end{array}$ & -8.2 & 10.3 & 25.0 & 13.2 & $\begin{array}{l}11.2 \\
11.5\end{array}$ & {$\left[\begin{array}{l}25.3 \\
25.0\end{array}\right.$} & & 0. \\
\hline \multirow[t]{2}{*}{4} & 18.5 & -17.6 & 0.9 & $\cdots$ & & $\cdots$ & 9.0 & -1.0 & 8.0 & 17.9 & -8.3 & 9.6 & 25.0 & $" 6$ & 11.5 & 25.8 & -14.3 & 11.5 \\
\hline & 18.3 & $"$ & 0.7 & & & & & & & & & & 24.4 & " & 10.9 & 25.9 & & 20. \\
\hline \multirow[t]{2}{*}{5} & 18.2 & $" 6$ & 0.6 & $\ldots$ & & $\cdots$ & 8.2 & -1.2 & 7.0 & 17.0 & -8.5 & 8.5 & 23.5 & " & 10.0 & 25.6 & -14.4 & 11.2 \\
\hline & 18.2 & “ & $0.6^{\circ}$ & & & & & & & 16.2 & -8.6 & 7.6 & & & & $25.1 \mid$ & & 10.7 \\
\hline \multirow[t]{2}{*}{6} & 18.3 & $"$ & 0.7 & $\ldots$ & & $\cdots$ & 8.5 & -1.5 & 7.0 & 15.6 & -8.7 & 6.9 & 22.1 & " & 8.6 & 24.3 & -14.5 & 9. \\
\hline & 18.4 & " & 0.8 & & & & & & & 16.0 & -8.9 & 7.1 & & & & & & \\
\hline 7 & $\begin{array}{l}18.3 \\
18.3\end{array}$ & $?$ & $\cdots$ & 15.2 & $?$ & $\cdots$ & $\begin{array}{l}7.7 \\
7.6\end{array}$ & $\begin{array}{l}-1.7 \\
-1.9\end{array}$ & $\begin{array}{l}6.0 \\
5.7\end{array}$ & 16.6 & -9.1 & 7.5 & 20.2 & " & 6.7 & 22.5 & -14.6 & 7.9 \\
\hline \multirow[t]{2}{*}{8} & $18 \cdot 3$ & $"$ & $\ldots$ & 15.4 & 6 & $\ldots$ & 7.6 & -2.0 & 5.6 & 17.0 & -9.4 & 7.6 & 19.3 & -13.6 & 5.7 & 21.0 & -14.7 & 6.3 \\
\hline & 18.5 & " & $-\cdots$ & & & & 9.8 & -2.2 & 7.6 & & & & 18.3 & & 4.7 & & & \\
\hline & 19,4 & “" & $\cdots$ & 16.0 & "6 & --- & 10.4 & -2.4 & 8.0 & 17.0 & -9.7 & 7.3 & $\begin{array}{l}18.0 \\
18.0\end{array}$ & 6 & $\begin{array}{l}4.4 \\
4.4\end{array}$ & 20.8 & -148 & \\
\hline 10 & 20.5 & " &.- & 17.6 & $"$ & --- & 11.6 & -2.5 & 8.8 & 17.6 & -10.0 & 7.6 & 18.0 & " & 4.4 & 19.8 & -14.9 & 4. \\
\hline 11 & 21.9 & $"$ & ... & 20.2 & $"$ & $\ldots$ & 13.6 & -3.2 & 10.4 & 18.2 & -10.2 & 8.0 & $\begin{array}{l}18.3 \\
19.2\end{array}$ & " & $\begin{array}{l}4.7 \\
5.6\end{array}$ & $\begin{array}{l}19.4 \\
19.2\end{array}$ & $\begin{array}{c}" 6 \\
-15.0\end{array}$ & t. \\
\hline Midn't & 23.3 & -17.6 & 5.7 & 23.2 & -17.0 & 6.2 & $15.7 ?$ & -3.6 & $(12.1)$ & 18.9 & -10.4 & 8.5 & 19.5 & -13.7 & 5.8 & $\begin{array}{l}19.4 \\
19.8\end{array}$ & -15.1 & 4.7 \\
\hline
\end{tabular}

Fath. Feet. Incli. Register.

Dec. 17. Sounding at noon $750 \quad 30.0$ changed to 23.0 . Results doubtful.

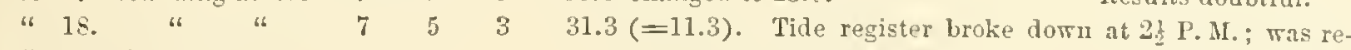

"19. No sounding taken. [paired and observations commenced at 7 P. M.

«20.

"21. Sounding at noon $7 \quad 3 \quad 6 \quad 21.5$. Correction at noon by soundings 12.3, by curves 14.5 , "22. " " $6 \quad 7 \quad 3 \quad 6 \quad 21.7$. Mean correction - 14.0. [mean adopted.

The heights on the 18 th and 19 th liare been rejected. 
Series I.-Timal Observations from October 10, 1853, to Decenber 28, 1853.

Hourly observations on the pulley-gange. Adopted reading of mean level 7.0 , expressed in units of the scale. Increasing numbers indicate rise of water.

December, 1853.

\begin{tabular}{|c|c|c|c|c|c|c|c|c|c|c|c|c|c|c|c|c|c|c|}
\hline $\begin{array}{l}\text { Mean } \\
\text { solar } \\
\text { bour. }\end{array}$ & 23d. & $\begin{array}{l}\text { fied. } \\
\text { to } \\
\text { level. }\end{array}$ & $\begin{array}{l}\text { ef. } \\
\text { bs. }\end{array}$ & 24 th. & $\begin{array}{c}\text { fed. } \\
\text { to } \\
\text { level. }\end{array}$ & $\begin{array}{l}\text { Ref. } \\
\text { obs. }\end{array}$ & 25 th. & $\begin{array}{c}\text { Red. } \\
\text { to } \\
\text { Jerel. }\end{array}$ & $\begin{array}{l}\text { Ref. } \\
\text { obs. }\end{array}$ & 20 th. & $\begin{array}{c}\text { Red. } \\
\text { to } \\
\text { lerel. }\end{array}$ & $\begin{array}{l}\text { Ref. } \\
\text { obs. }\end{array}$ & 27 th. & $\begin{array}{c}\text { Rerl. } \\
\text { to } \\
\text { lerel. }\end{array}$ & $\begin{array}{l}\text { Ref. } \\
\text { obs. }\end{array}$ & 25 th. & $\begin{array}{l}\text { Rerl. } \\
\text { to } \\
\text { lerel. }\end{array}$ & $\begin{array}{l}\text { Peff. } \\
\text { obs. }\end{array}$ \\
\hline 1 & 20.0 & -15.2 & 4.8 & 19.1 & -16.0 & 3.1 & $\begin{array}{l}19.5 \\
19.3 \\
19.3\end{array}$ & $\begin{array}{c}" \\
-16.9 \\
"\end{array}$ & $\begin{array}{l}2.7 \\
2.4 \\
2.4\end{array}$ & $\begin{array}{l}1.9 \\
1.8\end{array}$ & +1.4 & $\begin{array}{l}3.3 \\
3.2\end{array}$ & $\begin{array}{l}3.1 \\
2.7\end{array}$ & -0.4 & $\begin{array}{l}2.7 \\
2.3\end{array}$ & 7.1 & -2.0 & 5.1 \\
\hline 2 & 21.1 & -15.3 & 5.8 & 19.7 & "6 & 3.7 & 19.6 & -17.0 & 2.6 & $\begin{array}{l}1.8 \\
1.9\end{array}$ & $"$ & $\begin{array}{l}3.2 \\
3.3\end{array}$ & $\begin{array}{l}2.3 \\
2.7\end{array}$ & -0.5 & $\begin{array}{l}1.0 \\
2.8 \\
2.2\end{array}$ & 5.7 & -2.1 & 3.6 \\
\hline 3 & 22.0 & -15.4 & 6.6 & 21.0 & "6 & 5.0 & 20.3 & -17.1 & 3.2 & 2.1 & +1.3 & 3.4 & 3.5 & -0.6 & 2.9 & $\begin{array}{l}4.4 \\
4.1\end{array}$ & $\begin{array}{c}11 \\
-2.2\end{array}$ & $\begin{array}{l}2.3 \\
1.9\end{array}$ \\
\hline 4 & $22 . \bar{t}$ & -15.5 & 7.2 & 21.7 & -15.9 & 5.8 & 21.8 & -17.2 & 4.6 & 3.2 & +1.2 & 4.4 & ?3.0 & 66 & $? 2.4$ & $\begin{array}{l}4.2 \\
4.3\end{array}$ & $\begin{array}{c}6 \\
-2.3\end{array}$ & $\begin{array}{l}2.0 \\
2.0\end{array}$ \\
\hline 5 & $\begin{array}{l}23.0 \\
23.1\end{array}$ & -15.6 & $\begin{array}{l}7.4 \\
7.5\end{array}$ & 22.0 & $" 6$ & 6.1 & 23.4 & -17.3 & 6.1 & 5.6 & ،: & 6.8 & 4.7 & -0.7 & 4.0 & 4.7 & $"$ & 2.4 \\
\hline 6 & 23.1 & -15.7 & $\begin{array}{l}7.4 \\
7.0\end{array}$ & $\begin{array}{l}33.4 \\
23.6\end{array}$ & 6 & $\begin{array}{l}7.5 \\
7.7\end{array}$ & 24.9 & -17.4 & 7.5 & 6.7 & +1.0 & 7.7 & 6.6 & $" 6$ & 5.9 & 6.9 & -2.4 & 4.5 \\
\hline 7 & 23.0 & -15.8 & 7.2 & 23.6 & -15.8 & 7.8 & 25.3 & -17.5 & $\begin{array}{l}7.8 \\
8.0\end{array}$ & $\begin{array}{l}8.2 \\
8.7\end{array}$ & +0.9 & $\begin{array}{r}9.1 \\
9.6\end{array}$ & 8.5 & -0.8 & 7.7 & 9.2 & $" 6$ & 6.8 \\
\hline 8 & 22.4 & -15.9 & 6.5 & 22.9 & " & 7.1 & $\begin{array}{l}25.6 \\
25.6 \\
25.7\end{array}$ & -17.6 & $\begin{array}{l}8.0 \\
8.0\end{array}$ & $\begin{array}{r}9.2 \\
10.7 \\
0.9\end{array}$ & +0.8 & $\begin{array}{l}10.0 \\
11.5\end{array}$ & 10.6 & -0.9 & 9.7 & 11.1 & -2.5 & 8.6 \\
\hline 9 & 21.9 & -16.0 & 5.9 & 22.3 & "6 & 6.5 & 25.1 & $u$ & 7.5 & 9.8 & +0.7 & 10.5 & $\begin{array}{l}11.9 \\
12.2\end{array} \mid$ & -1.0 & $\begin{array}{l}10 . ? \\
11.2\end{array}$ & $\begin{array}{l}13.3 \\
13.8\end{array} \mid$ & " & $\begin{array}{l}10.8 \\
11.3\end{array}$ \\
\hline 10 & 20.6 & -10.1 & 4.5 & 21.2 & " & 5.4 & 23.6 & -17.7 & 5.9 & 8.5 & “ & 9.2 & $\begin{array}{l}12.1 \\
11.6\end{array}$ & -1.1 & $\begin{array}{l}11.0 \\
10.5\end{array}$ & $\begin{array}{l}14.2 \\
14.2\end{array}$ & -2.6 & 11.6 \\
\hline 11 & $\begin{array}{l}19.4 \\
19.3\end{array}$ & -16.2 & $\begin{array}{l}3.2 \\
3.1\end{array}$ & $\mid \begin{array}{l}21.0 \\
20.7\end{array}$ & " & $\begin{array}{l}5.2 \\
4.9\end{array}$ & $\begin{array}{l}22.9 \\
22.3\end{array}$ & -17.8 & $\begin{array}{l}5.1 \\
4.5\end{array}$ & 6.7 & +0.6 & 7.3 & 11.0 & -1.2 & 9.8 & 13.7 & " & 11.1 \\
\hline Noon : & 20.4 & -16.3 & 4.1 & 20.3 & -15.7 & $\begin{array}{l}4.6 \\
5.6\end{array}$ & $\begin{array}{l}22.0 \\
22.0\end{array}$ & -17.9 & $\begin{array}{l}4.1 \\
4.1\end{array}$ & 5.6 & +0.6 & 6.2 & 9.2 & -1.3 & 7.9 & 11.7 & -2.7 & 9.0 \\
\hline 1 & 21.4 & “ & 5.1 & 21.7 & " & 6.0 & 22.2 & -18.0 & 4.2 & $\begin{array}{l}4.4 \\
4.4\end{array}$ & $"$ & $\begin{array}{l}5.0 \\
5.0\end{array}$ & 6.9 & $"$ & 5.6 & 10.0 & -2.9 & 7.1 \\
\hline 2 & 22.5 & " & 6.2 & 22.5 & -15.8 & 6.7 & 22.5 & $"$ & 4.5 & $\begin{array}{l}4.3 \\
4.3\end{array}$ & +0.5 & $\begin{array}{l}4.8 \\
4.8\end{array}$ & 5.2 & -1.4 & 3.8 & 9.6 & -3.3 & 6.3 \\
\hline 3 & 23.6 & -16.2 & 7.4 & 23.5 & -15.9 & 7.6 & 23.2 & -18.1 & 5.1 & $\begin{array}{l}4.3 \\
4.3\end{array}$ & " & $\begin{array}{l}4.5 \\
4.8\end{array}$ & $\begin{array}{l}4.5 \\
4.4\end{array}$ & -1.5 & $\begin{array}{l}3.0 \\
2.9\end{array}$ & 9.5 & -3.8 & 5.7 \\
\hline 4 & {$\left[\begin{array}{l}24.6 \\
25.0 \\
25.0\end{array}\right.$} & $"$ & $\begin{array}{l}8.4 \\
-.5\end{array}$ & 21.5 & -16.0 & 8.5 & 23.6 & -18.2 & 5.4 & 45 & +0.4 & 4.9 & $\begin{array}{l}4.4 \\
4.4\end{array}$ & " & $\begin{array}{l}2.9 \\
2.9\end{array}$ & $\begin{array}{l}9.5 \\
9.6\end{array}$ & $\begin{array}{l}-4.5 \\
-4.7\end{array}$ & $\begin{array}{l}5.0 \\
4.9\end{array}$ \\
\hline 5 & $\begin{array}{l}24.3 \\
24.7\end{array}$ & $" 6$ & $\begin{array}{l}8.7 \\
8.5\end{array}$ & 25.1 & -16.1 & 9.0 & 24.6 & $"$ & 6.4 & 5.2 & +0.3 & 5.5 & 4.7 & -1.6 & 3.1 & $\mid \begin{array}{r}9.8 \\
10.0\end{array}$ & $\begin{array}{l}-4.9 \\
-5.1\end{array}$ & $\begin{array}{l}4.9 \\
4.9\end{array}$ \\
\hline 6 & 24.6 & "6 & 8.4 & $\begin{array}{l}25.4 \\
-5.5 .5\end{array}$ & -16.2 & $\begin{array}{l}9.2 \\
9.3\end{array}$ & 25.3 & -18.3 & 7.0 & 0.8 & " & 7.1 & 6.3 & " & 4.7 & 10.4 & -5.3 & 5.1 \\
\hline 7 & 23.7 & -16.1 & 7.6 & 25.1 & -16.3 & 8.8 & $\left|\begin{array}{l}25.3 \\
25.6\end{array}\right|$ & "6 & $\begin{array}{l}7.0 \\
7.3\end{array}$ & $\begin{array}{l}8.1 \\
8.5\end{array}$ & +0.2 & $\begin{array}{l}8.3 \\
8.7\end{array}$ & 7.8 & -1.7 & 6.1 & 12.6 & -5.7 & 6.9 \\
\hline 8 & 22.3 & 16 & 6.2 & 24.2 & -16.4 & 7.8 & 25.4 & -18.4 & 7.0 & $\begin{array}{l}9.1 \\
9.1\end{array}$ & $+\frac{10.1}{66}$ & $\begin{array}{l}9.2 \\
0.2\end{array}$ & 9.3 & "6 & 7.6 & 14.2 & -6.0 & 8.2 \\
\hline 9 & 20.1 & " & 4.0 & 23.1 & -16.5 & 6.6 & 25.0 & "6 & 6.6 & 9.1 & 0.0 & 9.1 & $\begin{array}{l}10.0 \\
10.9\end{array}$ & -1.8 & $\begin{array}{l}8.2 \\
9.1\end{array}$ & 16.3 & -6.3 & 10.0 \\
\hline 10 & 19.4 & “" & 3.3 & 22.61 & -16.6 & 6.11 & 24.1 & -18.5 & 5.6 & 8.1 & -0.1 & 8.0 & $\begin{array}{l}10.8 \\
10.4\end{array}$ & " & $\begin{array}{l}9.0 \\
8.6\end{array}$ & $\mid \begin{array}{l}17.1 \\
17.4\end{array}$ & $\begin{array}{l}-6.9 \\
-7.0\end{array}$ & $\begin{array}{l}10.2 \\
10.4\end{array}$ \\
\hline 11 & $\begin{array}{l}19.1 \\
19.0\end{array}$ & " & $\begin{array}{l}3.0 \\
2.9\end{array}$ & 22.1 & -16.7 & 5.4 & 23.1 & "t & 4.65 & 7.2 & -1.2 & 7.0 & 10.2 & -1.9 & 8.3 & $\begin{array}{l}17.7 \\
17.7\end{array}$ & $\begin{array}{l}-7.1 \\
-7.2\end{array}$ & $\begin{array}{l}10.6 \\
10.5\end{array}$ \\
\hline$M i \operatorname{In} t^{\prime}$ & 19.0 & -16.0 & 3.0 & 21.4 & -16.8 & .1 .6 & 22.4 & -18.6 & 3.8 & 5.2 & -0.3 & 4.9 & 9.0 & -2.0 & 7.0 & 17.7 & -7.3 & 10.4 \\
\hline I) Ee: & .23 & Sountir & ig at & noon & $\begin{array}{l}\text { Futh. } \\
6\end{array}$ & Fit & $\begin{array}{c}\text { Inch. } \\
8\end{array}$ & \multicolumn{9}{|c|}{ Correction. } & & \\
\hline " & 21. & " & " & 6 & 6 & 4 & 8 & -15.7 & & " & " & & $"$ & & & & & \\
\hline " & 25. & $"$ & " & ، & 6 & 3 & 0 & -17.9 & & 16 & " & & $"$ & & & & & \\
\hline " & 24. & " & " & 6 & 7 & 1 & 0 & +10.15 & & 6 & "6 & & $" 4$ & & & & & \\
\hline 16 & 27. & $"$ & " & " & $\tau$ & 2 & 0 & -1.3 & & 16 & $"$ & & 6 & & & & & \\
\hline " & 29. & $"$ & $"$ & ، & 7 & 4 & (i & (E)h] litl & 10 at? & $2+1$ & M.) & Jorrect & tion & -3.6 & tin & (n) & Irting & and \\
\hline
\end{tabular}
rurves; aftumon corrections from tho curves. IBotwen this tate and the commencement of the second series the whervations aro ton much affected by irregularities to be inserted. 
Semies II.-Tinal Onservations from Jandary 28 to A pric $九$, 1854.

Hourly observations on the pulley-gange. Adopted reading of mean level 7.0 , expressed in units of the seale. Inereasing numbers indicate rise of water.

\begin{tabular}{|c|c|c|c|c|c|c|c|c|c|c|c|c|c|c|c|c|c|c|}
\hline \multicolumn{13}{|c|}{ January, 1854.} & \multicolumn{6}{|c|}{ February, 1854.} \\
\hline $\begin{array}{l}\text { Mean } \\
\text { solar } \\
\text { hour. }\end{array}$ & $28 \mathrm{th}$. & $\begin{array}{c}\text { Red. } \\
\text { to } \\
\text { level. }\end{array}$ & $\begin{array}{l}\text { Ref. } \\
\text { obs. }\end{array}$ & 29 th. & $\begin{array}{c}\text { Rell. } \\
\text { to } \\
\text { level. }\end{array}$ & $\begin{array}{l}\text { Ref. } \\
\text { obs. }\end{array}$ & 30 th. & $\begin{array}{c}\text { Rerl. } \\
\text { to } \\
\text { level. }\end{array}$ & $\begin{array}{l}\text { Ref. } \\
\text { obs. }\end{array}$ & 3lst. & $\begin{array}{c}\text { led. } \\
\text { to } \\
\text { level. }\end{array}$ & $\begin{array}{l}\text { Ref. } \\
\text { obs. }\end{array}$ & 1st. & $\begin{array}{c}\text { Red. } \\
\text { to } \\
\text { level. }\end{array}$ & $\begin{array}{l}\text { Ref. } \\
\text { obs. }\end{array}$ & 21. & $\begin{array}{c}\text { Red. } \\
\text { to } \\
\text { level. }\end{array}$ & $\begin{array}{l}\text { Ref. } \\
\text { obs. }\end{array}$ \\
\hline 1 & $\begin{array}{l}13.2 \\
12.5\end{array}$ & $\begin{array}{l}-2.6 \\
-2.5\end{array}$ & $\begin{array}{l}10.6 \\
10.0\end{array} \mid$ & 21.8 & $\begin{array}{l}-10.0 \\
-10.1\end{array}$ & $\begin{array}{l}11.9 \\
11.4\end{array}$ & $\begin{array}{l}16.5 \\
16.2\end{array}$ & $\begin{array}{r}-7.5 \\
-7.4\end{array}$ & $\begin{array}{l}9.0 \\
8.8\end{array}$ & $\begin{array}{l}14.9 \\
15.5 \\
15.2\end{array}$ & $\begin{array}{c}-5.8 \\
" 6\end{array}$ & $\begin{array}{l}9.1 \\
9.7 \\
9.4\end{array}$ & 16.2 & -5.8 & 10.4 & $\begin{array}{l}13.2 \\
13.5\end{array}$ & -6.2 & $\begin{array}{l}7.0 \\
7.3\end{array}$ \\
\hline 2 & 12.0 & -3.3 & 8.7 & 19.6 & -10.2 & 9.4 & 15.6 & -7.3 & 8.3 & 14.7 & “ & 8.9 & $\begin{array}{l}16.5 \\
17.0\end{array}$ & -5.9 & $\left.\begin{array}{l}10.9 \\
11.1\end{array}\right]$ & 14.3 & " & 8.1 \\
\hline 3 & 11.0 & -3.8 & 7.2 & 17.7 & -10.3 & 7.4 & 12.8 & -7.2 & 5.6 & 14.3 & -5.7 & 8.6 & $\begin{array}{l}17.0 \\
17.0\end{array}$ & " 13 & $\begin{array}{l}11.1 \\
11.1\end{array}$ & $\begin{array}{l}15.6 \\
16.0\end{array}$ & $"$ " & $\begin{array}{l}9.4 \\
9.8\end{array}$ \\
\hline 4 & 8.8 & -4.2 & 4.6 & 16.1 & -10.4 & 5.7 & 10.5 & -7.1 & 3.4 & 11.7 & “ & 6.0 & 16.9 & -6.0 & 10.9 & $\begin{array}{l}16.2 \\
16.2\end{array}$ & “" & $\begin{array}{l}10.0 \\
10.0\end{array}$ \\
\hline 5 & $\begin{array}{l}6.3 \\
5.9\end{array}$ & $\begin{array}{r}-4.7 \\
-4.9\end{array}$ & $\begin{array}{l}1.6 \\
1.0\end{array}$ & 15.0 & -10.5 & 4.5 & 9.2 & -7.0 & 2.2 & 9.2 & " & 3.5 & 12.6 & “ & 6.6 & 15.7 & “ & 9.5 \\
\hline 6 & $\begin{array}{l}6.2 \\
6.7\end{array}$ & $\begin{array}{l}-5.1 \\
-5.3\end{array}$ & $\begin{array}{l}1.1 \\
1.4\end{array}$ & $\begin{array}{l}12.2 \\
11.9\end{array}$ & -10.6 & $\begin{array}{l}1.6 \\
1.3\end{array}$ & $\begin{array}{l}7.4 \\
7.4\end{array}$ & -6.9 & $\begin{array}{l}0.5 \\
0.5\end{array}$ & $\begin{array}{l}6.9 \\
5.9\end{array}$ & $\begin{array}{r}4 \\
-5.6\end{array}$ & $\begin{array}{l}1.2 \\
0.3\end{array}$ & 10.6 & -6.1 & 4.5 & 14.0 & “ & 7.8 \\
\hline 7 & 8.2 & -5.4 & 2.8 & $\begin{array}{l}12.1 \\
13.4\end{array}$ & -10.7 & $\begin{array}{l}1.4 \\
2.7\end{array}$ & \begin{tabular}{|l|}
7.5 \\
7.8
\end{tabular} & -6.8 & $\begin{array}{l}0.7 \\
1.0\end{array}$ & $\begin{array}{l}5.3 \\
5.3\end{array}$ & $"$ & $\begin{array}{l}-0.3 \\
-0.3\end{array}$ & $\begin{array}{l}8.5 \\
7.9\end{array}$ & $"$ " & $\begin{array}{l}2.4 \\
1.8\end{array}$ & 10.6 & “ & 4.4 \\
\hline 8 & 11.0 & -5.9 & 5.1 & 14.6 & -10.8 & 3.8 & 8.2 & -6.7 & 1.5 & $\begin{array}{l}5.3 \\
5.3\end{array}$ & $\begin{array}{c}u \\
-5.5\end{array}$ & $\begin{array}{l}-0.3 \\
-0.2\end{array}$ & $\begin{array}{l}7.7 \\
7.7\end{array}$ & $"$ & $\begin{array}{l}1.6 \\
1.6\end{array}$ & $\begin{array}{l}8.9 \\
9.1\end{array}$ & "“ & $\begin{array}{l}2.7 \\
2.9\end{array}$ \\
\hline 9 & 15.6 & -6.4 & 9.2 & 18.6 & -10.9 & 7.7 & 10.3 & -6.6 & 3.7 & 6.9 & " & 1.4 & $\begin{array}{l}7.5 \\
8.3\end{array}$ & -6.2 & $\begin{array}{l}1.3 \\
2.1\end{array}$ & $\begin{array}{l}8.2 \\
8.2\end{array}$ & $"$ & $\begin{array}{l}2.0 \\
2.0\end{array}$ \\
\hline 10 & 17.7 & -7.2 & 10.5 & 20.7 & -11.0 & 9.7 & 12.2 & -6.5 & 5.7 & 10.0 & “ & 4.5 & 10.0 & " & 3.8 & 8.7 & " & 2.5 \\
\hline 11 & 19.9 & $-S .0$ & 11.9 & 23.4 & -11.1 & 12.3 & 14.3 & -6.4 & 7.9 & 13.0 & -5.4 & 7.6 & 13.0 & “ & 6.8 & 11.2 & $"$ & 5.0 \\
\hline Noon & $\left|\begin{array}{l}21.0 \\
21.2\end{array}\right|$ & -8.8 & $\begin{array}{l}12.2 \\
12.4\end{array}$ & 24.5 & $\begin{array}{l}-11.2 \\
-11.1\end{array}$ & $\begin{array}{r}13.3 \\
9.6\end{array}$ & 18.3 & -6.3 & 12.0 & $\begin{array}{l}16.2 \\
17.3\end{array} \mid$ & -5.3 & $\begin{array}{l}10.9 \\
12.0\end{array}$ & 16.3 & -6.3 & 10.0 & 13.0 & -6.1 & 6.9 \\
\hline 1 & 20.4 & -8.9 & 11.5 & $\begin{array}{l}19.7 \\
19.2\end{array}$ & $\begin{array}{l}-11.0 \\
-10.9\end{array}$ & $\begin{array}{l}8.7 \\
8.3\end{array}$ & $\begin{array}{l}20.1 \\
20.2\end{array}$ & $"$ & $\begin{array}{l}13.8 \\
13.9\end{array}$ & $\begin{array}{l}18.3 \\
19.0\end{array}$ & " & $\begin{array}{l}13.0 \\
13.7\end{array}$ & 17.7 & “ & 11.4 & 14.6 & " & 8.5 \\
\hline 2 & 18.8 & -9.0 & 9.8 & 18.7 & -10.8 & 7.9 & 19.7 & -6.2 & 13.5 & 18.2 & -5.4 & 12.8 & $\begin{array}{l}18.4 \\
19.0\end{array}$ & "“ & $\begin{array}{l}12.1 \\
12.7\end{array}$ & $\begin{array}{l}16.0 \\
16.2\end{array}$ & "“ & $\begin{array}{r}9.9 \\
10.1\end{array}$ \\
\hline 3 & 17.1 & -9.1 & 8.0 & 15.6 & -10.6 & 5.0 & 17.7 & -6.1 & 11.6 & 16.5 & " & 11.4 & $\begin{array}{l}19.2 \\
18.9\end{array}$ & "“ & $\begin{array}{l}12.9 \\
12.6\end{array}$ & $\begin{array}{l}16.7 \\
16.0\end{array}$ & " " & $\begin{array}{r}10.6 \\
9.9\end{array}$ \\
\hline 4 & 15.6 & -9.2 & 6.4 & 12.0 & -10.4 & 1.6 & 14.6 & $"$ & 8.5 & 15.7 & -5.5 & 10.2 & 18.2 & " & 11.9 & 16.0 & " & 9.9 \\
\hline 5 & 13.7 & -9.3 & 4.4 & 10.8 & -10.2 & 0.6 & 8.2 & -6.0 & 2.2 & 13.0 & $"$ & 7.5 & 15.8 & " & 9.5 & 14.9 & -6.2 & 8.7 \\
\hline 6 & $\mid \begin{array}{l}12.2 \\
12.0\end{array}$ & -9.4 & $\begin{array}{l}2.8 \\
2.6\end{array}$ & $\begin{array}{r}10.0 \\
9.7\end{array}$ & $\begin{array}{l}-10.0 \\
-9.9\end{array}$ & $\begin{array}{r}0.0 \\
-0.2\end{array}$ & $\begin{array}{l}6.3 \\
6.1\end{array}$ & "، & $\begin{array}{l}0.3 \\
0.1\end{array}$ & 9.7 & -5.6 & 4.1 & 12.4 & " & 6.1 & 13.9 & " & 7.7 \\
\hline 7 & 12.2 & -9.5 & 2.7 & $\begin{array}{l}9.2 \\
8.6\end{array}$ & $\begin{array}{l}-9.8 \\
-9.7\end{array}$ & $\begin{array}{r}-0.6 \\
-1.1\end{array} \mid$ & $\begin{array}{l}5.7 \\
5.6\end{array}$ & " & $\begin{array}{l}-0.3 \\
-0.3\end{array}$ & $\begin{array}{l}7.3 \\
7.2\end{array}$ & " & $\begin{array}{l}1.7 \\
1.6\end{array}$ & 10.0 & “ & 3.7 & 10.5 & " & 4.6 \\
\hline 8 & 14.2 & -9.6 & 4.6 & $\begin{array}{l}7.8 \\
7.7\end{array}$ & $\begin{array}{l}-9.6 \\
-9.5\end{array}$ & $\begin{array}{l}-1.8 \\
-1.8\end{array}$ & $\begin{array}{l}5.5 \\
5.7\end{array}$ & "“ & $\begin{array}{l}-0.4 \\
-0.2\end{array}$ & $\begin{array}{l}7.2 \\
7.2\end{array}$ & $\begin{array}{c}" \\
-5.7\end{array}$ & $\begin{array}{l}1.0 \\
1.6 \\
1.5\end{array}$ & $8 . \overline{7}$ & “" & $\begin{array}{l}2.4 \\
2.2\end{array}$ & 8.1 & “ & 1.9 \\
\hline$\because$ & 17.1 & -9.7 & 7.4 & 8.7 & -9.4 & -0.7 & 6.7 & $"$ & 0.8 & $\begin{array}{l}7.4 \\
8.0\end{array}$ & $"$ " & $\begin{array}{l}1.7 \\
2.3\end{array}$ & $\begin{array}{l}8.4 \\
8.4\end{array}$ & "، & $\begin{array}{l}2.1 \\
2.1\end{array}$ & \begin{tabular}{|l|}
6.6 \\
6.6
\end{tabular} & " & $\begin{array}{l}0.4 \\
0.4\end{array}$ \\
\hline 10 & 19.9 & -9.8 & 10.1 & 10.8 & -92 & 0.6 & 9.3 & " & 3.4 & 9.0 & “ & 3.3 & 8.6 & " & 2.3 & $\begin{array}{l}6.6 \\
6.6\end{array}$ & -6.3 & $\begin{array}{l}0.3 \\
0.3\end{array}$ \\
\hline 11 & 21.1 & -9.9 & 11.2 & 13.4 & -9.0 & 4.4 & 12.1 & " & 6.2 & 12.0 & " & 6.3 & 10.0 & “ & 3.7 & 7.2 & “ & 0.9 \\
\hline Midn't & 21.6 & -10.0 & 11.6 & 14.8 & -8.7 & 0.1 & 14.0 & -5.8 & 8.2 & 14.6 & -5.8 & 8.8 & 12.1 & -6.2 & 5.9 & 9.3 & $" 6$ & 3.0 \\
\hline
\end{tabular}

Jan. 27. At 11 P. MI., 13.7; at $11^{\mathrm{h}} 30^{\mathrm{m}}, 13.8$; at $28 \mathrm{tb}, 0^{\mathrm{h}}, 13.9$, ligh water; colrected high water 11.3.

Fath. Feet. Inch.

an. 28. Sounding at noon $8 \quad 0 \quad 0 \quad$ Corrected reading by sonnding 11.4, by curres 13.1, mean 12.2.

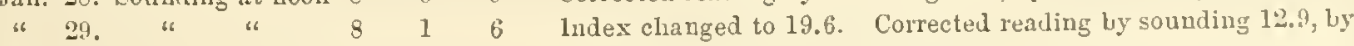
curves 13.7, mean 13.3 .

"30. " " 8 " 6 " 6 Corrected reading by sounding 11.9 , by curves 12.1 , mean 12.0 .

"31. " " $\quad$ "...

Feb. 1. " " $\quad 7 \quad 4 \quad 6$ Ebb tide at $7 \frac{1}{2} \mathrm{~A}$. M.

"2. " " 250100 Ebb tideat 4 P. M. (probably means ebb commences). Inder 13.0 Note to Feb. 1 and 2. The correctiou is derived from the soundings and curves. 
Series II.-Tinal Observations fron January 28 to A prid 7, 1854.

Hourly observations ou the pulley-gange. Adopted reading of mean level 7.0, expressed in units of the scale. Increasing numbers indieate rise of water.

\section{February, 1854.}




Series II.-Tidal Onservations From January 28 to A pril 7, 1854.

IIourly observations on the pulley-gauge. Adopted leading of mean level 7.0, expressed in units of the scale. Increasing numbers indicate rise of water.

\section{February, 1854.}

\begin{tabular}{|c|c|c|c|c|c|c|c|c|c|c|c|c|c|c|c|c|c|c|}
\hline $\begin{array}{l}\text { Mean } \\
\text { solar } \\
\text { bour. }\end{array}$ & h. & $\begin{array}{c}\text { Red. } \\
\text { to } \\
\text { level. }\end{array}$ & ef. & I0th. & $\begin{array}{c}\text { lied. } \\
\text { to } \\
\text { level. }\end{array}$ & $\begin{array}{l}\text { lief. } \\
\text { obs. }\end{array}$ & tith. & $\begin{array}{c}\text { Red. } \\
\text { to } \\
\text { level. }\end{array}$ & $\begin{array}{l}\text { Ref. } \\
\text { olss. }\end{array}$ & 12 th. & $\begin{array}{c}\text { Red. } \\
\text { to } \\
\text { level. }\end{array}$ & ef. & $13 \mathrm{th}$. & $\begin{array}{c}\text { Red. } \\
\text { to } \\
\text { level. }\end{array}$ & $\begin{array}{l}\text { Ref. } \\
\text { obs. }\end{array}$ & $14 t b$. & $\begin{array}{c}\text { Red. } \\
\text { to } \\
\text { level. }\end{array}$ & $\begin{array}{l}\text { Ref. } \\
\text { obs. }\end{array}$ \\
\hline 1 & 9.9 & -4.6 & 5.3 & 11.4 & -5.6 & 5.8 & 10.7 & -6.3 & 4.4 & 11.1 & -6.2 & 4.8 & & -6.7 & $\begin{array}{l}8.0 \\
7.5\end{array}$ & 19.5 & -9.4 & 10. \\
\hline 2 & $\begin{array}{l}8.5 \\
8.4\end{array}$ & "4 & $\begin{array}{l}4.2 \\
3.8\end{array}$ & 11.0 & -5.7 & 5.3 & 10.1 & $"$ & 3.8 & 11.1 & " & 4.9 & 13.6 & " & 6.9 & 16.5 & -9.6 & \&. \\
\hline 3 & $\begin{array}{l}8.4 \\
8.4\end{array}$ & $"$ & $\begin{array}{l}3.8 \\
3.8\end{array}$ & 10.4 & -5.8 & 4.6 & 8.8 & $"$ & 2.5 & 10.2 & " & 4.0 & 12.7 & -6.8 & 5.9 & 16.0 & -9.8 & 6.2 \\
\hline 4 & $\begin{array}{l}8.4 \\
8.4\end{array}$ & " & $\begin{array}{l}3.8 \\
3.8\end{array}$ & 10.0 & -5.9 & 4.1 & $\begin{array}{l}8.2 \\
7.9\end{array}$ & " & $\begin{array}{l}1.9 \\
1.65\end{array}$ & $\begin{array}{l}9.0 \\
9.5\end{array}$ & " "s & $\begin{array}{l}2.5 \\
3.3\end{array}$ & 9.7 & " & 2.9 & 14.0 & -10.0 & 4.0 \\
\hline 5 & 8. & " & 4.2 & 10.0 & -6.0 & 4.0 & $\begin{array}{l}7.5 \\
7.5\end{array}$ & -6.2 & $\begin{array}{l}1.3 \\
1.3\end{array}$ & $\begin{array}{l}3.2 \\
9.0\end{array}$ & " & $\begin{array}{l}3.0 \\
2.8\end{array}$ & $\begin{array}{l}8.2 \\
8.0\end{array}$ & " & $\begin{array}{l}1.4 \\
1.2\end{array} \mid$ & 13.7 & -10.1 & 3. \\
\hline 6 & 10.0 & " & 5.4 & $\begin{array}{r}10.0 \\
9.8 \\
1.8\end{array}$ & -6.1 & $\begin{array}{l}3.9 \\
3.7\end{array}$ & 7.8 & " & 1.6 & \begin{tabular}{|l|}
9.0 \\
9.0 \\
\end{tabular} & $"$ & $\begin{array}{l}2.8 \\
38\end{array}$ & $\begin{array}{l}7.6 \\
8.5\end{array}$ & -6.9 & $\begin{array}{l}0.7 \\
1.6\end{array}$ & $\left|\begin{array}{|l|}13.3 \\
12.9\end{array}\right|$ & & $\begin{array}{l}3.0 \\
2.5\end{array}$ \\
\hline 7 & 11.6 & $"$ & 7.01 & 11.0 & -6.2 & 4.8 & 9.1 & " & 2.9 & 9.7 & " & 3.5 & 8.9 & “ & 2.0 & \begin{tabular}{|l|}
12.9 \\
13.7
\end{tabular} & & \\
\hline 8 & 12.8 & " & 8.2 & 12.8 & -6.3 & 6.5 & 10.3 & -6.1 & 4.2 & 11.2 & " & 5.0 & 10.9 & " & 4.0 & 14.7 & -10.7 & 4. \\
\hline 9 & 13.5 & " & 8.9 & $\begin{array}{l}15.0 \\
15.2\end{array} \mid$ & -6.4 & $\begin{array}{l}8.6 \\
8.8\end{array}$ & 12.6 & " & 6.5 & 13.2 & " & 7.0 & 13.0 & -7.0 & 6.0 & 16.2 & -10.8 & \\
\hline 10 & & " & $\begin{array}{l}9.5 \\
9.7\end{array}$ & $\left|\begin{array}{l}15.7 \\
15.7\end{array}\right|$ & -6.5 & $\begin{array}{l}9.2 \\
9.2\end{array}$ & 15.5 & " & 9.4 & & " " & $\begin{array}{r}9.1 \\
10.4\end{array}$ & & "6 & $\begin{array}{l}9.0 \\
9.9\end{array}$ & 18.7 & -10.9 & \\
\hline 11 & $\begin{array}{l}14.2 \\
13.8\end{array}$ & & & & $"$ " & $\begin{array}{l}9.2 \\
8.5\end{array}$ & $\begin{array}{l}16.1 \\
16.4\end{array}$ & $"$ & & $\begin{array}{l}17.7 \\
17.4\end{array}$ & & & & " & $\begin{array}{l}12.1 \\
12.6\end{array}$ & 20.8 & -11.1 & • \\
\hline Noon & 13.6 & -4.5 & 9.1 & 14.0 & -6.6 & 7.4 & $\begin{array}{l}16.5 \\
16.1\end{array}$ & -6.0 & $\begin{array}{l}105 \\
10.1\end{array}$ & 17.1 & -6.3 & 10.8 & \begin{tabular}{|l|}
19.0 \\
19.5 \\
19.5
\end{tabular} & $\begin{array}{l}-7.1 \\
-7.2\end{array}$ & $\begin{array}{l}12.4 \\
12.3\end{array}$ & 21.7 & -11.2 & 10.5 \\
\hline 1 & 13.5 & " & 9.0 & 13.9 & “ & 7.3 & 15.7 & " & 9.7 & 16.0 & “ & 9.7 & 19.5 & -7.3 & 12.2 & $\left|\begin{array}{l}24.8 \\
-4.3\end{array}\right|$ & " & $\begin{array}{l}13 . \\
13 .\end{array}$ \\
\hline 2 & 12.7 & $"$ & 8.2 & 13.4 & "s & 6.8 & 13.6 & " & 7.6 & 14.7 & " & 8.4 & 17.9 & -7.5 & 10.4 & 23.2 & -11.3 & 11. \\
\hline 3 & 11.2 & -4.6 & 6.6 & 10.7 & " & 4.1 & 11.4 & " & 5.4 & 12.3 & -6.4 & 5.9 & 16.7 & -7.7 & 9.0 & 21.5 & -11.4 & 10.1 \\
\hline 4 & 8. & -4.7 & $\begin{array}{l}4.3 \\
3.6\end{array}$ & $\begin{array}{l}8.7 \\
8.0\end{array}$ & -6.5 & $\begin{array}{l}2.2 \\
1.5\end{array}$ & $\begin{array}{l}9 . \\
8 .\end{array}$ & " & $\begin{array}{l}3.5 \\
2.0\end{array}$ & 10. & " & 3.6 & & $\begin{array}{l}-7.8 \\
-8.0\end{array}$ & & 19.5 & “ & \\
\hline 5 & $\begin{array}{l}8.0 \\
8.7\end{array}$ & "4.8 & $\begin{array}{l}3.3 \\
3.9\end{array}$ & & $"$ " & & $\begin{array}{l}7.8 \\
8.0\end{array}$ & " & $\begin{array}{l}1.8 \\
2.0\end{array}$ & 9.7 & " & 3.3 & $\begin{array}{l}12.9 \\
12.3\end{array}$ & $\begin{array}{l}-8.1 \\
-8.2\end{array}$ & & 16.7 & -11.5 & \\
\hline 6 & 9.3 & & 4.5 & $\begin{array}{l}8.0 \\
8.0\end{array}$ & " " & $\begin{array}{l}1.5 \\
1.5\end{array}$ & 8.0 & " & 2.0 & $\begin{array}{l}8.9 \\
88\end{array}$ & -6.5 & $\begin{array}{l}2.4 \\
2.3\end{array}$ & 13.1 & -8.3 & 4.8 & $\begin{array}{l}14.7 \\
14.3\end{array}$ & 16 & \\
\hline 7 & 9.7 & -4.9 & 4.8 & 8.5 & $"$ & 2.0 & 9.6 & " & 3.6 & $\begin{array}{l}8 . \\
8 .\end{array}$ & " & $\begin{array}{l}2.3 \\
2.4\end{array}$ & 15.2 & -8.4 & 6.8 & $\begin{array}{l}14.2 \\
14.2\end{array}$ & -11.6 & \\
\hline 8 & 11.0 & -5.0 & 6.0 & 9.8 & -6.4 & 3.4 & 10.3 & -6.1 & 4.2 & 9.7 & " & 3.2 & 16.3 & -8.5 & 7.8 & 14.6 & " & \\
\hline 8 & 11.7 & -5.1 & 6.6 & 11.2 & " & 4.8 & 11.4 & " & 5.3 & 9.9 & -6.6 & 3.3 & 16.3 & -8.7 & 7.6 & 15.1 & “ & o. \\
\hline 10 & 12.4 & -5.2 & 7.2 & 12.3 & " & 5.9 & 12.1 & " & 6.0 & $\begin{array}{l}12 . \\
14.3\end{array}$ & st & $\begin{array}{l}6.0 \\
7.7\end{array}$ & & $\begin{array}{l}-8.8 \\
-8.9\end{array}$ & $\begin{array}{r}9.9 \\
10.2\end{array}$ & 15.8 & -11.7 & \\
\hline 11 & $\begin{array}{l}12.3 \\
12.6\end{array}$ & & $\begin{array}{l}7.0 \\
7.2\end{array}$ & $\begin{array}{l}12.7 \\
12.7\end{array}$ & & $\begin{array}{l}6.3 \\
6.3\end{array}$ & & " & $\begin{array}{l}6.5 \\
7.2\end{array}$ & $\begin{array}{l}15.0 \\
15.0\end{array}$ & "s & $\begin{array}{l}8.4 \\
8.4\end{array}$ & $\begin{array}{l}19.6 \\
19.5\end{array}$ & $\begin{array}{l}-9.1 \\
-9.1\end{array}$ & $\begin{array}{l}10.6 \\
10.4\end{array}$ & 16.6 & & \\
\hline Midn't & 12.4 & -5.5 & 6.9 & 12.9 & -6.3 & 6.6 & 13.8 & " & 7.7 & 15.1 & -6.7 & 8.4 & 19.5 & -9.2 & 10.3 & 17.4 & -11.8 & 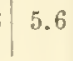 \\
\hline
\end{tabular}

Feb. 9. No sounding.

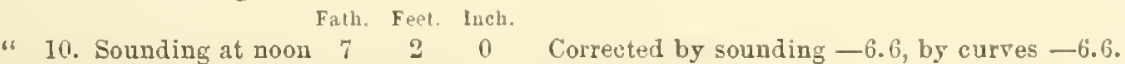

"11. " "

" 12. " " $\quad 7 \quad 4 \quad 6$ Ebb tide at 6 P. M. Corrected by sounding -6.6 , by curres

-6.0 , mean -6.3 .

" $13.4 \quad$ " $\quad 8 \quad 1 \quad 6 \quad$ Corrected by sounding -6.6 , by curres -7.6 , mean -7.1 .

"14. No sounding. 
Series 11.-Tidal Obserfations from Jandary 28 to A Pril 7, 1854.

Hourly obselvations on the pulley-gaage. Adopted reading of mean level 7.9, expressed in units of the scale. Increasing numbers indicate rise of water.

February, 1854.

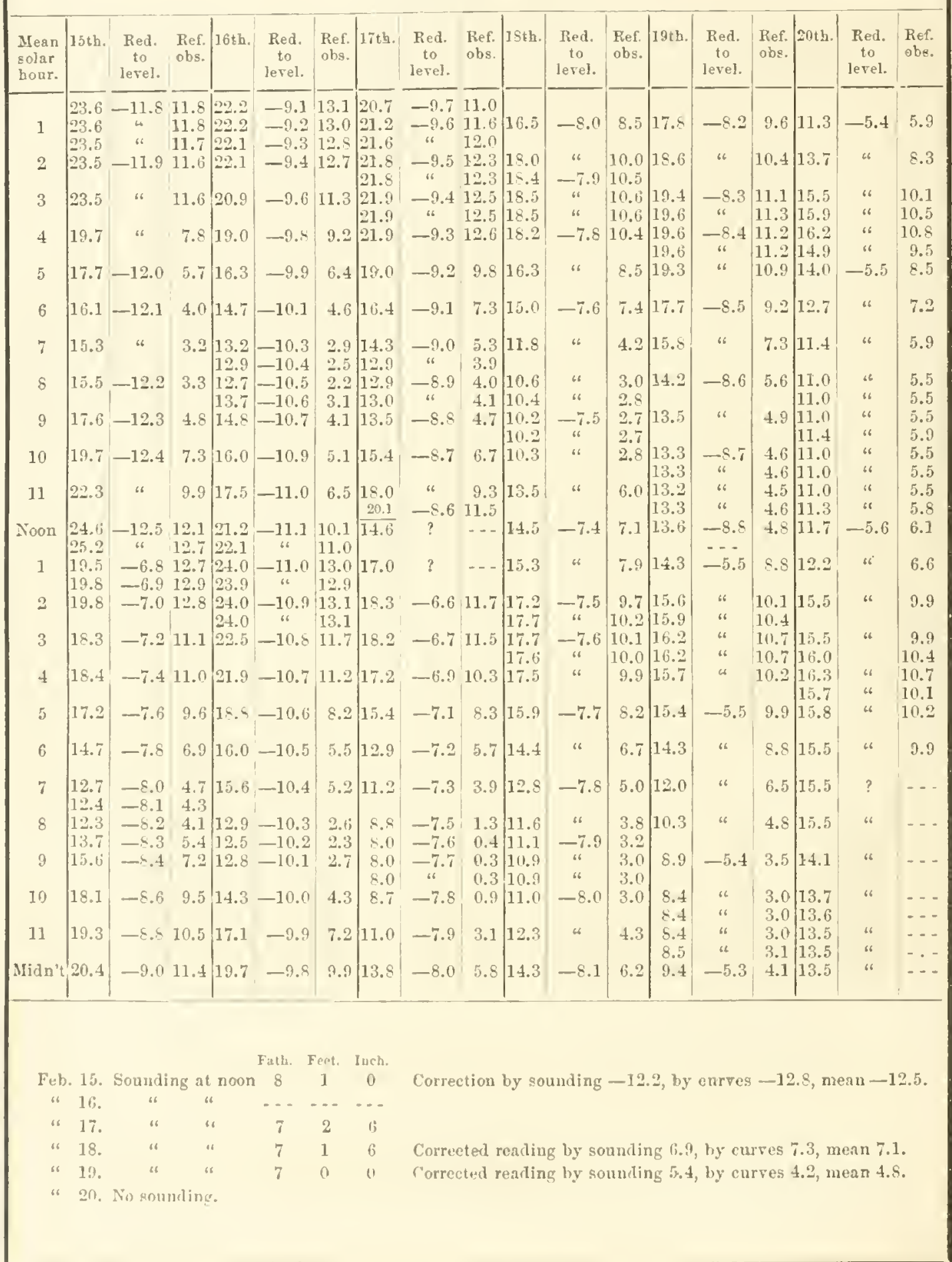


Series II.-Tidal Orseryations from January 28 to A prit $7,1854$.

Hourly observations on the pulley-gange. Adopted reading of mean level 7.0 , expressed in units of the seale. Inereasing numbers indiente rise of watcr.

\section{February, 1854.}

\begin{tabular}{|c|c|c|c|c|c|c|c|c|c|c|c|c|c|c|c|c|c|c|}
\hline $\begin{array}{l}\text { Mean } \\
\text { solar } \\
\text { bour. }\end{array}$ & st. & $\begin{array}{c}\text { Red. } \\
\text { to } \\
\text { lerel. }\end{array}$ & $\begin{array}{l}\text { Ref. } \\
\text { obs. }\end{array}$ & $22 \mathrm{~d}$. & $\begin{array}{c}\text { Red. } \\
\text { to } \\
\text { level. }\end{array}$ & $\begin{array}{l}\text { Ref. } \\
\text { obs. }\end{array}$ & $23 d$. & $\begin{array}{c}\text { Red. } \\
\text { to } \\
\text { level. }\end{array}$ & $\begin{array}{l}\text { Ref. } \\
\text { obs. }\end{array}$ & 24th. & $\begin{array}{c}\text { Red. } \\
\text { to } \\
\text { level. }\end{array}$ & $\begin{array}{l}\text { Ref. } \\
\text { obs }\end{array}$ & 25 th. & $\begin{array}{c}\text { Rerl. } \\
\text { to } \\
\text { lovel. }\end{array}$ & $\begin{array}{l}\text { Ref. } \\
\text { obs. }\end{array}$ & $26 \mathrm{th}$. & $\begin{array}{c}\text { Red. } \\
\text { to } \\
\text { level. }\end{array}$ & $\begin{array}{l}\text { Ref. } \\
\text { bs. }\end{array}$ \\
\hline 1 & & & $\ldots$ & & -6.0 & $\begin{array}{l}4.5 \\
4.7\end{array}$ & $\begin{array}{l}12.4 \\
11.8\end{array}$ & $\begin{array}{l}-7.2 \\
-7.3\end{array}$ & $\begin{array}{l}5.2 \\
4.5\end{array}$ & $\begin{array}{l}14.4 \\
14.2\end{array}$ & -10.9 & $\begin{array}{l}3.5 \\
3.3\end{array}$ & 9.8 & -2.1 & 7.7 & 30.6 & -2.2 & 8.4 \\
\hline 2 & & & & 11.5 & " & 5.5 & $\mid \begin{array}{l}11.8 \\
11.8\end{array}$ & $\begin{array}{l}-7.4 \\
-7.5\end{array}$ & $\begin{array}{l}4.4 \\
4.3\end{array}$ & 14.5 & -11.0 & 3.5 & 7.8 & " & 5.7 & 8.8 & -2.4 & 6.4 \\
\hline 3 & & & $\cdots$ & 12.9 & " & 6.9 & $\begin{array}{l}11.8 \\
12.1\end{array}$ & $\begin{array}{l}-7.7 \\
-7.8\end{array}$ & $\begin{array}{l}4.1 \\
4.3\end{array}$ & 14.9 & -11.1 & 3.8 & $\begin{array}{l}5.8 \\
5.3\end{array}$ & -2.2 & $\begin{array}{l}3.6 \\
3.1\end{array}$ & 6.4 & -2.7 & 3.7 \\
\hline 4 & & & $\cdots$ & 14.2 & " & 8.2 & 13.0 & -8.0 & 5.0 & 16.1 & -11.2 & 4.9 & $\begin{array}{r}5.1 \\
15.4\end{array}$ & -2.3 & $\begin{array}{l}2.8 \\
3.1\end{array}$ & $\begin{array}{l}5.2 \\
4.1\end{array}$ & $\begin{array}{l}-3.0 \\
-3.1\end{array}$ & $\begin{array}{l}2.2 \\
1.0\end{array}$ \\
\hline 5 & $\cdots$ & & $\cdots$ & 14.1 & ? & & 14.3 & -8.2 & 6.1 & 17.2 & -11.3 & 5.9 & 5.6 & “ & .3 & $\begin{array}{l}4.2 \\
4.3\end{array}$ & $\begin{array}{l}-3.2 \\
-3.3\end{array}$ & $\begin{array}{l}1.0 \\
1.0\end{array}$ \\
\hline 6 & $\ldots$ & & $\cdots$ & 13.6 & $"$ & $\ldots$ & 15.8 & -8.3 & 7.5 & 18.3 & -11.5 & 6.8 & 7.6 & -2.4 & 5.2 & 4.7 & -3.5 & 1. \\
\hline 7 & & & & 13.2 & " & .. & 16.8 & -8.5 & 8.3 & 19.4 & -11.6 & 7.8 & 9.7 & " & 7.3 & 6.6 & -3.7 & 2. \\
\hline 8 & & & & $\begin{array}{l}12.7 \\
15.0\end{array}$ & $\begin{array}{c}" \\
-6.2\end{array}$ & 3.8 & 18.1 & -8.6 & 9.5 & 21.7 & -11.8 & 9.2 & 11.0 & -2.5 & 8.5 & 9.0 & -4.0 & 5. \\
\hline 9 & $\ldots$ & & & $\begin{array}{l}15.5 \\
15.6\end{array}$ & " & $\begin{array}{l}9.3 \\
9.4\end{array}$ & 18.4 & -8.7 & 9.7 & $\begin{array}{l}22.2 \\
22.2\end{array}$ & -11.9 & $\begin{array}{l}10.3 \\
10.3\end{array}$ & 12.7 & $"$ & 10.2 & 11.0 & -4.3 & .7 \\
\hline 10 & $\ldots$ & & -- & 14.9 & “: & 8.7 & $\begin{array}{l}19.1 \\
19.5\end{array}$ & -8.8 & $\begin{array}{l}10.3 \\
10.7\end{array}$ & 22.3 & -12.0 & 10.3 & $\begin{array}{l}13.8 \\
13.8\end{array}$ & -2.6 & $\begin{array}{l}11.2 \\
11.2\end{array}$ & 13.0 & -4.6 & 8.4 \\
\hline 11 & - - & & $\ldots$ & 14.2 & " & 8.0 & 19.3 & -8.9 & 10.4 & 22.2 & -12.1 & 10.1 & 13.8 & " & 11.2 & 14.6 & -4.9 & 9.7 \\
\hline Noou & .. & & $\ldots$ & 12.8 & -6.2 & 6.6 & 18.7 & -9.0 & 9.7 & 20.7 & -12.3 & 8.4 & 13.7 & -2.7 & 11.0 & 15.4 & -5.4 & \\
\hline 1 & $\cdots$ & & & 12.2 & " & 6.0 & 17.9 & -9.3 & 8.6 & 10.0 & -1.7 & 8.3 & 11.4 & -1.9 & 9.5 & & $\begin{array}{l}-5.3 \\
-5.6 \\
-5.7\end{array}$ & \\
\hline 2 & $\cdots$ & & & 12.2 & " & 6.0 & 16.7 & -9.6 & 7.1 & 7.6 & & 5.9 & 9.0 & -1.0 & 8.0 & 15.0 & -5.8 & 9. \\
\hline 3 & & & & 12.2 & -6.3 & 5.9 & & $\begin{array}{r}-9.9 \\
-10.0\end{array}$ & $\begin{array}{l}6.2 \\
6.0\end{array}$ & $\begin{array}{l}6.6 \\
5.0\end{array}$ & -1.8 & & 6.0 & -1.1 & 4.9 & 13.1 & -6.0 & 7. \\
\hline 4 & $-\ldots$ & & & $\begin{array}{l}12.5 \\
11.0\end{array}$ & " & $\begin{array}{l}6.2 \\
4.7\end{array}$ & & -10.2 & $\begin{array}{l}5.8 \\
5.8\end{array}$ & $\begin{array}{l}4.6 \\
5.3\end{array}$ & $"$ & $\begin{array}{l}2.8 \\
3.5\end{array}$ & 4.7 & & $\begin{array}{l}3.4 \\
2.0\end{array}$ & 10.2 & -6.1 & 4.7 \\
\hline 5 & & & & $\begin{array}{l}11.7 \\
12.9\end{array}$ & -6.4 & $\begin{array}{l}5.3 \\
6.5\end{array}$ & $\begin{array}{l}16 \\
16\end{array}$ & " & $\begin{array}{l}5.7 \\
6.4\end{array}$ & 5.6 & " & 3.8 & 3.7 & -1.4 & 2.3 & $\begin{array}{l}9.5 \\
9.4\end{array}$ & $\begin{array}{l}-6.2 \\
-6.3\end{array}$ & $\begin{array}{l}3 . \\
3 .\end{array}$ \\
\hline 6 & & & & 14.2 & “ & 7.8 & 17.0 & -10.3 & 6.7 & 6.4 & " & 4.6 & 4.1 & -1.5 & 2.6 & $\begin{array}{l}9.4 \\
9.5\end{array}$ & $\begin{array}{l}-6.4 \\
-6.5\end{array}$ & $\begin{array}{l}3 . \\
3 .\end{array}$ \\
\hline 7 & & & & $\begin{array}{l}15.1 \\
15.2\end{array}$ & -6.5 & $\begin{array}{l}8.6 \\
8.7\end{array}$ & 18.0 & 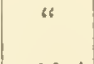 & 7.7 & 7.7 & 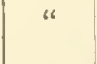 & 5.9 & 5.4 & -1.6 & 3.5 & 9.8 & -6.6 & 3. \\
\hline 8 & 13.5 & -5.8 & 7.7 & $\begin{array}{l}15.7 \\
15.6\end{array}$ & -6.6 & $\begin{array}{l}9.1 \\
9.0\end{array}$ & $\begin{array}{l}18.9 \\
19.3\end{array}$ & -10.4 & $\begin{array}{l}8.5 \\
8.9\end{array}$ & 9.6 & -1.9 & 7.7 & 8.0 & -1.7 & 0.3 & 11.5 & -6.7 & 4. \\
\hline 9 & 12.5 & & 6.7 & $\begin{array}{l}15.3 \\
15.1\end{array}$ & -6.7 & $\begin{array}{l}8.6 \\
7.4\end{array}$ & $\begin{array}{l}19.3 \\
19.3\end{array}$ & -10.5 & $\begin{array}{l}8.8 \\
8.8\end{array}$ & & " & $\begin{array}{l}8.9 \\
9.4\end{array}$ & 10.2 & -1.8 & 8.4 & 15.0 & -6.8 & 8.2 \\
\hline 10 & 11.2 & -5.9 & 5.3 & 15.0 & -6.8 & 8.2 & 19.0 & -10.6 & 8.4 & $\begin{array}{l}11.6 \\
11.6\end{array}$ & $"$ & $\begin{array}{l}9.7 \\
9.7\end{array}$ & $\begin{array}{l}11.9 \\
12.3\end{array}$ & -1.9 & $\begin{array}{l}10.0 \\
10.4\end{array}$ & 16.3 & -6.9 & 9.4 \\
\hline 11 & & " " & $\begin{array}{l}4.5 \\
4.6\end{array}$ & 14.6 & -6.9 & 7.7 & 18.5 & -10.7 & 7.8 & & & $\begin{array}{l}9.7 \\
9.7\end{array}$ & $\begin{array}{l}12.4 \\
12.4\end{array}$ & -2.0 & $\begin{array}{l}10.4 \\
10.4\end{array}$ & 17.8 & -7.0 & 10.8 \\
\hline Midn't & 10.2 & “ & 4.3 & 13.5 & -7.0 & 6.8 & 16.4 & -10.8 & 5.6 & $10.6^{2}$ & -2.0 & $8.6^{\circ}$ & 12.3 & -2.0 & 10.3 & 18.4 & -7.1 & 11.3 \\
\hline
\end{tabular}

Feb. 21. Readings irregular, tide-gauge out of order at 8 A. M., repaired at noon. Sounding $7 \mathrm{fath}, \mathrm{ft}_{\mathrm{f}}, 0 \mathrm{in}$. Feb. 22 and 23. No sounding.

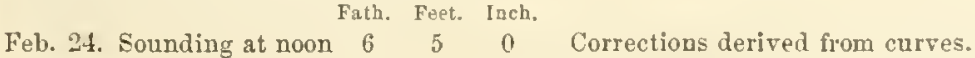

"25. " " 700 G Ebb tide at $11 \mathrm{~A}$. M. Corrections deriven from curres.

"26. " " $\quad 7 \quad 5 \quad 6 \quad$ Correction derived from the means by sounding and curres. 
Series II.-Tidal Orservations from Jandary 28 to Apriı $7,1854$.

Honrly observations on the pulley-gange. Adopted reading of mean level 7.0, expressed in units of the scale. Inereasing numbers indicate rise of water.

\begin{tabular}{|c|c|c|c|c|c|c|c|c|c|c|c|c|c|c|c|c|c|c|}
\hline \multicolumn{7}{|c|}{ February, 1854.} & \multicolumn{12}{|c|}{ March, 1854.} \\
\hline $\begin{array}{l}\text { Mean } \\
\text { solar } \\
\text { hour. }\end{array}$ & $2 \pi$ th. & $\left\{\begin{array}{c}\text { Red. } \\
\text { to } \\
\text { lerel. }\end{array}\right.$ & $\begin{array}{l}\text { Ref. } \\
\text { obs. }\end{array}$ & $28 \mathrm{th}$. & $\begin{array}{c}\text { Red. } \\
\text { to } \\
\text { level. }\end{array}$ & $\begin{array}{l}\text { Ref. } \\
\text { obs. }\end{array}$ & Ist. & $\begin{array}{c}\text { Red. } \\
\text { to } \\
\text { lerel. }\end{array}$ & $\begin{array}{l}\text { Ref. } \\
\text { obs. }\end{array}$ & $2 \mathrm{~d}$. & $\begin{array}{c}\text { Red. } \\
\text { to } \\
\text { level. }\end{array}$ & $\begin{array}{l}\text { Ref. } \\
\text { obs. }\end{array}$ & $3 \mathrm{~d}$. & $\begin{array}{c}\text { Red. } \\
\text { to } \\
\text { level. }\end{array}$ & $\begin{array}{l}\text { Ref. } \\
\text { obs. }\end{array}$ & 4th. & $\begin{array}{c}\text { Red. } \\
\text { to } \\
\text { level. }\end{array}$ & $\begin{array}{l}\text { Ref. } \\
\text { obs. }\end{array}$ \\
\hline 1 & 18.3 & -7.3 & 11.0 & $\begin{array}{l}15.0 \\
14.8\end{array}$ & -4.9 & $\begin{array}{r}10.1 \\
9.9\end{array}$ & \begin{tabular}{|l|}
15.5 \\
16.3
\end{tabular} & -5.0 & $\begin{array}{l}10.5 \\
11.3\end{array}$ & & $\begin{array}{l}-5.0 \\
" 6\end{array}$ & 11.2 & $\begin{array}{l}14.5 \\
15.5 \\
16\end{array}$ & $\begin{array}{c}-5.4 \\
"\end{array}$ & $\begin{array}{r}9.1 \\
10.1 \\
10.1\end{array}$ & 13.1 & -5.1 & 8.0 \\
\hline 2 & 17.5 & -7.4 & 10.1 & 12.8 & “ & 7.9 & 16.3 & " & 11.3 & $\begin{array}{l}17.0 \\
17.0 \\
16.5\end{array}$ & $" 6$ & $\begin{array}{l}12.0 \\
12.0 \\
11.5\end{array}$ & $\begin{array}{l}16.0 \\
16.0 \\
16.0\end{array}$ & $"$ & $\begin{array}{l}10.6 \\
10.6 \\
10.6\end{array} \mid$ & $\begin{array}{l}15.4 \\
15.9\end{array}$ & -5.0 & $\begin{array}{l}10.4 \\
10.9\end{array}$ \\
\hline 3 & 17.8 & -7.5 & 10.3 & 11.0 & " & 6.1 & 13.9 & " & 8.9 & 16.0 & " & 11.0 & $\begin{array}{l}16.0 \\
15.7\end{array}$ & " & $\begin{array}{l}10.6 \\
10.3\end{array}$ & $\begin{array}{l}16.0 \\
16.0\end{array}$ & -4.9 & $\begin{array}{l}11.1 \\
11.1\end{array}$ \\
\hline 4 & $\begin{array}{r}19.3 \\
5.3\end{array}$ & $\stackrel{?}{4} .8$ & - . & 7.8 & " & 2.9 & 12.0 & “ & 7.0 & 15.1 & " & 10.1 & 14.8 & -5.5 & 9.3 & 15.8 & 6 & 10.9 \\
\hline 5 & $\begin{array}{l}4.4 \\
4.4\end{array}$ & $"$ & $\begin{array}{l}-0.4 \\
-0.4\end{array}$ & $\begin{array}{l}5.5 \\
5.5\end{array}$ & $"$ " & $\begin{array}{l}0.6 \\
0.6\end{array}$ & 10.5 & " & 5.5 & 12.8 & " & 7.8 & 13.6 & " & 8.1 & $\mid 14.2$ & -4.5 & 9.4 \\
\hline 6 & 5.0 & " & 0.2 & $\begin{array}{l}5.0 \\
5.0\end{array}$ & "“ & $\begin{array}{l}0.1 \\
0.1\end{array}$ & $\begin{array}{l}7.2 \\
5.5\end{array}$ & " " & $\begin{array}{l}2.2 \\
0.5\end{array}$ & 11.1 & " & 6.1 & 12.4 & “ & 6.9 & 12.1 & " & 7.3 \\
\hline 7 & 5.8 & " & 1.0 & $\begin{array}{l}5.0 \\
6.2\end{array}$ & "“ & $\begin{array}{l}0.1 \\
1.3\end{array}$ & $\begin{array}{l}5.5 \\
5.5\end{array}$ & “ & $\begin{array}{l}0.5 \\
0.5\end{array}$ & $\begin{array}{l}9.0 \\
8.8\end{array}$ & "“ & $\begin{array}{l}4.0 \\
3.8\end{array}$ & 11.1 & -5.6 & 5.5 & $\begin{array}{l}9.7 \\
9.1\end{array}$ & -4.7 & $\begin{array}{l}5.0 \\
4.4\end{array}$ \\
\hline 8 & 8.3 & " & 3.5 & 7.0 & " & 2.1 & $\begin{array}{l}5.0 \\
5.5 \\
5.7\end{array}$ & " " & $\begin{array}{l}0.5 \\
0.7\end{array}$ & 7.5 & "، & 2.5 & $\begin{array}{l}9.4 \\
8.1\end{array}$ & “" & $\begin{array}{l}3.5 \\
2.5\end{array}$ & $\begin{array}{l}8.6 \\
8.7\end{array}$ & “" & $\begin{array}{l}3.9 \\
4.0\end{array}$ \\
\hline 9 & 11.1 & $"$ & 6.3 & 10.0 & “ & 5.1 & 7.0 & " & 2.0 & 8.2 & “ & 3.2 & 9.0 & “ & 3.4 & 9.0 & " & 4.3 \\
\hline 10 & 14.4 & “ & 9.6 & 12.9 & " & 8.0 & 3.5 & “ & 4.5 & 10.0 & “ & 5.0 & 9.7 & -5.7 & 4.0 & 8.4 & " & 3.7 \\
\hline 11 & $\begin{array}{l}16.3 \\
16.9\end{array}$ & “ & $\begin{array}{l}11.5 \\
12.1\end{array}$ & 16.0 & “ & 11.1 & 13.5 & “ & 8.5 & 12.8 & “ & 7.8 & 11.2 & " & 5.5 & $\begin{array}{l}8.7 \\
9.7\end{array}$ & $"$ & $\begin{array}{l}4.0 \\
5.0\end{array}$ \\
\hline Noon & $\begin{array}{l}17.6 \\
16.0\end{array}$ & -4.9 & $\begin{array}{l}12.7 \\
11.1\end{array}$ & $\begin{array}{l}17.7 \\
17.9\end{array}$ & -4.9 & $\begin{array}{l}12.8 \\
13.0\end{array}$ & 16.1 & -5.0 & 11.1 & 15.6 & -5.0 & 10.6 & 13.3 & -5.8 & 7.5 & 12.1 & -4.6 & 7.5 \\
\hline 1 & 15.3 & " & 10.4 & $\begin{array}{l}17.9 \\
17.2\end{array}$ & " & $\begin{array}{l}13.0 \\
12.3\end{array}$ & $\begin{array}{l}17.6 \\
17.9\end{array}$ & "، & $\begin{array}{l}12.6 \\
12.9\end{array}$ & & ": & $\begin{array}{l}12.9 \\
13.0\end{array}$ & 15.7 & " & 9.9 & 13.5 & " & 8.9 \\
\hline 2 & 14.1 & " & 9.2 & 15.9 & " & 11.0 & $\begin{array}{l}17 . \\
17 .\end{array}$ & "، & $\begin{array}{l}12.9 \\
12.2\end{array}$ & 18.0 & " & 13.0 & $\left|\begin{array}{l}16.0 \\
16.6\end{array}\right|$ & -5.7 & $\begin{array}{l}10.3 \\
10.9\end{array}$ & $\begin{array}{l}15.3 \\
15.5\end{array}$ & " & $\begin{array}{l}10.7 \\
10.9\end{array}$ \\
\hline 3 & 12.8 & " & 7.9 & 13.4 & " & 8.5 & 16.7 & " & 11.7 & 17.2 & -5.1 & 12.1 & & $"$ " & $\begin{array}{l}10.6 \\
10.6\end{array}$ & $\begin{array}{l}15.6 \\
15.2\end{array}$ & -4.5 & $\begin{array}{l}11.1 \\
10.7\end{array}$ \\
\hline 4 & 8.7 & " & 3.8 & 9.7 & " & 4.8 & 13.9 & " & 8.9 & 15.8 & " & 10.7 & 15.3 & " & 9.6 & 15.0 & “ & 10.5 \\
\hline 5 & $\begin{array}{l}4.7 \\
4.5\end{array}$ & $"$ & $\begin{array}{l}-0.2 \\
-0.4\end{array}$ & 7.8 & " & 2.9 & 12.4 & $"$ & 7.4 & 14.4 & -5.2 & 9.2 & 14.9 & -5.6 & 9.3 & 12.9 & -4.4 & 8.5 \\
\hline 6 & $\begin{array}{l}4.0 \\
4.0\end{array}$ & " " & $\begin{array}{l}-0.3 \\
-0.3\end{array}$ & & "“ & & 11.7 & " & 6.7 & 13.4 & " & 8.2 & 14.2 & 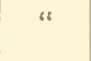 & 8.6 & 11.2 & " & 6.8 \\
\hline 7 & 4.7 & " & -0.2 & $\begin{array}{l}6.0 \\
6.0\end{array}$ & " " & $\begin{array}{l}1.1 \\
1.1\end{array}$ & 11.1 & “ & 6.1 & \begin{tabular}{|l|}
12.0 \\
11.3
\end{tabular} & "، & $\begin{array}{l}6.8 \\
0.1\end{array}$ & 13.5 & -5.5 & 8.0 & 9.9 & " & 5.5 \\
\hline 8 & 6.5 & " & 1.6 & $\begin{array}{l}6.0 \\
6.4\end{array}$ & " " & $\begin{array}{l}1.1 \\
1.5\end{array}$ & $\begin{array}{l}9.4 \\
8.5\end{array}$ & " & & $\begin{array}{r}10.0 \\
7.7\end{array}$ & -5.3 & 4.7 & $\begin{array}{r}12.1 \\
8.6\end{array}$ & " & $\begin{array}{c}6.6 \\
3.1\end{array}$ & $\begin{array}{l}8.2 \\
6.4\end{array}$ & -4.3 & $\begin{array}{l}3.9 \\
2.1\end{array}$ \\
\hline 9 & 8.9 & " & 4.0 & 8.0 & $"$ & 3.1 & \begin{tabular}{|l|}
6.3 \\
7.2
\end{tabular} & " " & $\begin{array}{l}1.3 \\
2.2\end{array}$ & $\begin{array}{l}7.5 \\
7.2\end{array}$ & " " & $\begin{array}{l}2.2 \\
1.9\end{array}$ & $\begin{array}{l}6.9 \\
6.9\end{array}$ & $\begin{array}{c}-5.4 \\
16\end{array}$ & $\begin{array}{l}1.5 \\
1.5\end{array}$ & $\begin{array}{l}5.2 \\
5.3\end{array}$ & -4.2 & $\begin{array}{l}1.0 \\
1.1\end{array}$ \\
\hline 10 & 11.6 & " & 6.7 & 10.4 & “ & 5.5 & 8.9 & " & 3.9 & 8.5 & " & 3.2 & 7.2 & " & 1.8 & 5.3 & " & 1.1 \\
\hline 11 & $\begin{array}{l}13.8 \\
14.7\end{array}$ & " & $\begin{array}{l}8.9 \\
9.8\end{array}$ & $\begin{array}{l}15.8 \\
17.0\end{array}$ & $"$ " & & 11.8 & " & 6.8 & 11.2 & 66 & 5.9 & 9.2 & -5.3 & 3.9 & $\begin{array}{l}0.0 \\
6.4\end{array}$ & 16 & 2.3 \\
\hline Midn't & 15.2 & " & 10.3 & 17.3 & " & 12.4 & 14.6 & $"$ & 9.6 & 13.9 & -5.4 & 8.5 & 11.4 & -5.2 & 6.2 & 8.6 & -4.0 & 4.6 \\
\hline
\end{tabular}

Fath. Feot. ln lis.

Fef. 27. Sounding at noon 7 ; (; Mean correction ly sounding and curves adopted, the latter 28. No sounding. [showing weight 2 . IIarch 1. No soumling.

2. Sounding at noon 7 b) (i) Corrected reading by sounding 11.0 , by curves 10.2, moan 10.6 . 3. " $" 7 \quad 6$ Corrected reading by sounding 7.0 , by curves 8.0, mean 7.5 4. No soundins. 
Series II.-Tidal Observations from January 28 to A phil 7, 1854.

IIourly observations on the pulley-gange. Adopted reading of mean level 7.0 , expressed in units of the scale. Increasing numbers indicate rise of water.

March, 1854.

\begin{tabular}{|c|c|c|c|c|c|c|c|c|c|c|c|c|c|c|c|c|c|c|}
\hline $\begin{array}{l}\text { Mean } \\
\text { solar } \\
\text { hour. }\end{array}$ & 5 th. & $\begin{array}{c}\text { Red. } \\
\text { to } \\
\text { level. }\end{array}$ & $\begin{array}{l}\text { Ref. } \\
\text { obs. }\end{array}$ & Bth. & $\begin{array}{c}\text { Red. } \\
\text { to } \\
\text { level. }\end{array}$ & $\begin{array}{l}\text { Ref. } \\
\text { obs. }\end{array}$ & 7 th. & $\begin{array}{c}\text { Red. } \\
\text { to } \\
\text { level. }\end{array}$ & $\begin{array}{l}\text { Ref. } \\
\text { obs. }\end{array}$ & 10th. & $\begin{array}{c}\text { Red. } \\
\text { to } \\
\text { level. }\end{array}$ & $\begin{array}{l}\text { Ref. } \\
\text { obs. }\end{array}$ & IIth. & $\begin{array}{c}\text { Red. } \\
\text { to } \\
\text { level. }\end{array}$ & $\begin{array}{l}\text { Ref. } \\
\text { obs. }\end{array}$ & I5tb. & $\begin{array}{c}\text { Red. } \\
\text { to } \\
\text { level. }\end{array}$ & $\begin{array}{l}\text { Ref. } \\
\text { obs. }\end{array}$ \\
\hline 1 & 10.5 & -3.8 & 6.7 & 10.7 & -3.5 & 7.2 & 9.7 & -4.1 & 5.6 & 9.6 & -4.7 & 4.9 & $\begin{array}{l}15.1 \\
14.3\end{array}$ & -6.9 & $\begin{array}{l}8.2 \\
7.4\end{array}$ & $\mid \begin{array}{l}17.6 \\
17.5\end{array}$ & $\begin{array}{l}-6.4 \\
-6.5\end{array}$ & $\begin{array}{l}11.2 \\
11.0\end{array}$ \\
\hline 2 & 11.8 & " & 8.0 & 12.1 & " & 8.6 & 11.0 & " & 6.9 & $\begin{array}{l}9.1 \\
8.9 \\
8.9\end{array}$ & -4.8 & $\begin{array}{l}4.4 \\
4.1 \\
\end{array}$ & 13.2 & -7.0 & 6.2 & 15.7 & -6.7 & 9.0 \\
\hline 3 & $\begin{array}{l}12.7 \\
12.8\end{array}$ & -3.7 & $\begin{array}{l}9.0 \\
9.1\end{array}$ & 13.2 & -3.6 & 9.6 & 12.1 & " & 8.0 & $\begin{array}{l}8.9 \\
8.9 \\
9.0\end{array}$ & -4.9 & $\begin{array}{l}.1 \\
4.0 \\
4.1\end{array}$ & $\left|\begin{array}{l}12.5 \\
12.4\end{array}\right|$ & " " & $\begin{array}{l}5.5 \\
5.4\end{array}$ & 15.2 & -6.9 & 8.3 \\
\hline 4 & $\begin{array}{l}12.8 \\
11.9\end{array}$ & -3.6 & $\begin{array}{l}9.2 \\
5.3\end{array}$ & $\begin{array}{l}14.0 \\
14.1\end{array}$ & " & $\begin{array}{l}10.4 \\
10.5\end{array}$ & $\left|\begin{array}{|c|}12.8 \\
12.9\end{array}\right|$ & “ & $\begin{array}{l}8.7 \\
8.8\end{array}$ & $\begin{array}{l}9.0 \\
9.0\end{array}$ & -5.0 & $\begin{array}{l}4.0 \\
4.0\end{array}$ & $\begin{array}{l}12.4 \\
12.4\end{array}$ & "، & $\begin{array}{l}5.4 \\
5.4\end{array}$ & 14.0 & -7.1 & 6.9 \\
\hline 5 & $\begin{array}{l}11.7 \\
11.2\end{array}$ & -3.5 & $\begin{array}{l}8.2 \\
7.7\end{array}$ & $\left|\begin{array}{l}14.1 \\
14.0\end{array}\right|$ & -3.7 & $\begin{array}{l}10.4 \\
10.3\end{array}$ & $\begin{array}{l}12.9 \\
12.9\end{array}$ & $"$ & $\begin{array}{l}8.8 \\
8.8\end{array}$ & $\begin{array}{l}9.0 \\
9.0 \\
9.6\end{array}$ & $\begin{array}{l}-5.1 \\
-5.2\end{array}$ & $\begin{array}{l}3.9 \\
4.4\end{array}$ & $\begin{array}{l}12.4 \\
12.4\end{array}$ & "، & $\begin{array}{l}5.4 \\
5.4 \\
5.4\end{array}$ & 11.5 & -7.3 & 4.2 \\
\hline 6 & 10.9 & -3.4 & 7.5 & 13.9 & " & 10.2 & $\begin{array}{l}12.9 \\
12.9\end{array}$ & -1.2 & $\begin{array}{l}8.7 \\
8.7\end{array}$ & 10.0 & -5.3 & 4.7 & $\begin{array}{l}12.4 \\
12.5\end{array}$ & $"$ & $\begin{array}{l}5.4 \\
5.5\end{array}$ & $\begin{array}{l}10.2 \\
10.0\end{array}$ & $\begin{array}{l}-7.5 \\
-7.7\end{array}$ & $\begin{array}{l}2.7 \\
2.3\end{array}$ \\
\hline 7 & 9.7 & " & 6.3 & $|12.9|$ & -3.8 & 9.1 & $\begin{array}{l}12.9 \\
12.9\end{array}$ & $"$ & $\begin{array}{l}8.7 \\
8.7\end{array}$ & 11.3 & -5.4 & 5.9 & 12.8 & " & 5.8 & 10.7 & -7.9 & 2.8 \\
\hline 8 & $\begin{array}{l}8.7 \\
7.4\end{array}$ & -3.3 & $\begin{array}{l}5.4 \\
4.1\end{array}$ & 12.2 & " & 8.4 & $\begin{array}{l}12.9 \\
12.7\end{array}$ & " & $\begin{array}{l}8.7 \\
8.5\end{array}$ & 13.1 & -5.5 & 7.6 & 14.2 & " & 7.2 & 12.4 & -8.1 & 4.3 \\
\hline 9 & $\begin{array}{l}6.3 \\
6.6\end{array}$ & -3.2 & $\begin{array}{l}3.1 \\
3.4\end{array}$ & $\begin{array}{l}10.1 \\
10.0\end{array}$ & -3.9 & $\begin{array}{l}6.2 \\
6.1\end{array}$ & 12.1 & " & 7.9 & $\begin{array}{l}15.2 \\
15.5\end{array}$ & $\begin{array}{l}-5.6 \\
-5.6\end{array}$ & $\begin{array}{l}9.6 \\
9.9\end{array}$ & 16.0 & " & 9.0 & 17.0 & -8.3 & 8.7 \\
\hline 10 & $\begin{array}{l}6.6 \\
6.7\end{array}$ & -3.1 & $\begin{array}{l}3.5 \\
3.6\end{array}$ & $\begin{array}{l}10.0 \\
10.0\end{array}$ & " & $\begin{array}{l}6.1 \\
6.1\end{array}$ & $\mid \begin{array}{l}10.5 \\
10.5\end{array}$ & " & $\begin{array}{l}6.3 \\
6.3\end{array}$ & $\begin{array}{l}15.5 \\
15.5\end{array}$ & -5.7 & \begin{tabular}{l|}
9.8 \\
9.8
\end{tabular} & $\begin{array}{l}17.2 \\
17.5\end{array}$ & " " & $\begin{array}{l}10.2 \\
10.5\end{array}$ & 19.2 & -8.5 & 10.7 \\
\hline 11 & 7.3 & " & 4.2 & $\begin{array}{l}10.0 \\
10.4\end{array}$ & " & $\begin{array}{l}6.1 \\
6.5\end{array}$ & $\begin{array}{l}10.0 \\
10.2\end{array}$ & " & $\begin{array}{l}5.8 \\
6.0\end{array}$ & 15.3 & -5.8 & 9.51 & 17.6 & "6 & 10.6 & $\begin{array}{l}20.7 \\
21.5\end{array} \mid$ & $\begin{array}{l}-8.7 \\
-8.8\end{array}$ & $\begin{array}{l}12.0 \\
12.7\end{array}$ \\
\hline Noon & 9.5 & -3.0 & 6.5 & 10.4 & -4.0 & 6.4 & 10.2 & -4.3 & 5.9 & 15.0 & -5.9 & 9.1 & $\cdots$ & & & 18.0 & -8.9 & 9.1 \\
\hline 1 & 10.5 & " & 7.5 & 11.3 & “ & 7.3 & 10.5 & “ & 6.2 & 14.0 & -6.0 & 8.0 & & & & 18.3 & -9.0 & 9.3 \\
\hline 2 & $\mid \begin{array}{l}11.8 \\
12.3\end{array}$ & -3.1 & $\mid \begin{array}{l}8.7 \\
9.2\end{array}$ & 12.0 & " & 8.0 & & & & 11.9 & -6.1 & 5.8 & & & & 17.3 & -9.1 & 8.2 \\
\hline 3 & $\begin{array}{l}13.2 \\
13.2\end{array}$ & " & $\begin{array}{l}10.1 \\
10.1\end{array}$ & 12.6 & " & 8.6 & & & & $\begin{array}{l}11.5 \\
11.4\end{array}$ & -6.2 & $\begin{array}{l}5.3 \\
5.2\end{array}$ & & & & 16.0 & -9.2 & 6.8 \\
\hline 4 & $\begin{array}{l}13.2 \\
12.7\end{array}$ & -3.2 & $\begin{array}{r}10.0 \\
9.5\end{array}$ & 12.9 & " & 8.9 & $\stackrel{\frac{3}{3}}{30}$ & & & $\mid$\begin{tabular}{|l|}
11.3 \\
11.3
\end{tabular} & $\begin{array}{c}“ 6 \\
-6.3\end{array}$ & $\begin{array}{l}5.1 \\
5.0\end{array}$ & 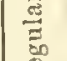 & & & 13.7 & " & 4.5 \\
\hline 5 & 11.9 & " & 8.7 & $\begin{array}{l}13.3 \\
13.6\end{array} \mid$ & $"$ " & $\begin{array}{l}9.3 \\
0.6\end{array}$ & $\stackrel{0}{\Xi}$ & & & $\begin{array}{l}11.3 \\
11.6\end{array}$ & " & $\begin{array}{l}5.0 \\
5.3\end{array}$ & 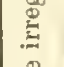 & & & 12.9 & -9.3 & 3.6 \\
\hline 6 & 10.4 & -3.3 & 7.1 & 13.3 & " & 9.3 & हू & & & 11.8 & -6.4 & 5.4 & ‡ & & & $\left|\begin{array}{l}12.6 \\
12.4\end{array}\right|$ & -0.4 & $\begin{array}{l}3.2 \\
3.0\end{array}$ \\
\hline 7 & 9.8 & " & 6.5 & 12.7 & "؛ & 8.7 & $\mathscr{D}_{0}^{\circ}$ & & & 12.3 & -6.5 & 5.8 & $\underbrace{0}_{0}$ & & & $\begin{array}{l}12.3 \\
12.3\end{array}$ & -9.5 & $\begin{array}{l}2.8 \\
2.5\end{array}$ \\
\hline 8 & 8.8 & " & 5.5 & 12.2 & " & 8.2 & 氖 & & & 12.9 & " & 6.4 & 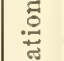 & & & \begin{tabular}{|l|}
12.3 \\
12.3
\end{tabular} & -9.6 & $\begin{array}{l}2.7 \\
2.7\end{array}$ \\
\hline 9 & $\begin{array}{l}7.7 \\
7.4\end{array}$ & -3.4 & $\begin{array}{l}4.3 \\
4.0\end{array}$ & 9.5 & “ & 5.5 & : & & & 13.5 & -6.6 & 6.9 & 苟 & & & 14.0 & -9.7 & 4.3 \\
\hline 10 & $\begin{array}{l}7.3 \\
7.5\end{array}$ & $"$ & $\begin{array}{l}3.9 \\
4.1\end{array}$ & $\begin{array}{l}8.2 \\
8.1\end{array}$ & $"$ " & $\begin{array}{l}4.2 \\
4.1\end{array}$ & 0 & & & $\begin{array}{l}15.0 \\
15.5\end{array}$ & -6.7 & $\begin{array}{l}8.3 \\
8.8\end{array}$ & 5 & & & 16.5 & -9.8 & 6.7 \\
\hline 11 & 7.8 & $"$ & 4.4 & $\begin{array}{l}8.1 \\
8.4\end{array}$ & $"$ & $\begin{array}{l}4.1 \\
4.4\end{array}$ & & & & $\begin{array}{l}15.5 \\
15.5\end{array}$ & $"$ & $\begin{array}{l}8.8 \\
8.8\end{array}$ & & & & 19.1 & -9.9 & 9.2 \\
\hline idn't & 9.2 & -3.5 & 5.7 & 8.6 & -4.1 & 4.5 & & & & 15.5 & -6.8 & 8.7 & & & & 20.5 & -10.0 & 10.5 \\
\hline
\end{tabular}

March 5. Soundings at noon 6 fath, 3 feet, 6 inches. Correction from curres.

Mareh 6. No somnding.

7. Tide register broke at $9 \mathrm{~A}$. $\mathrm{H}$., was repaired immediately. No sounding.

Narch 8. No sounding.

Fath. Feet. 1nch

"9. Sounding at moon 64 (1)

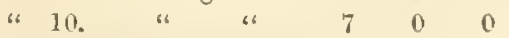

"11. " " " 70 "

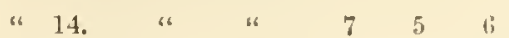

March 12. No sounding and but a fer observations taken. "13. But few observations taken.

The corrections after March 7 are derived from curves. 
Series II. - Tidal Observations fron JaNuary 28 to A Pril $7,1854$.

Honrly obserrations on the pulles-gange. Adopted rcading of mean level 7.0, expressed in units of the scale. Increasing numbers indicate rise of water.

\section{March, 1854.}

Mean 16th. Red. Ref. 17th. Red. Ref. 1Sth. Red. Ref. 19th. Red. Ref. 20th. Red. Ref. 23d. Red. Ref.

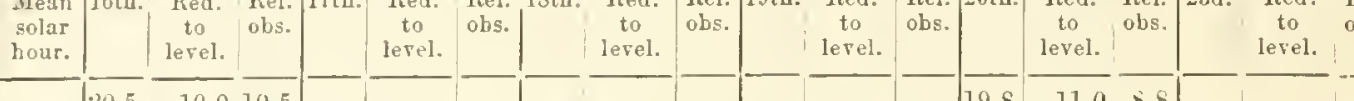

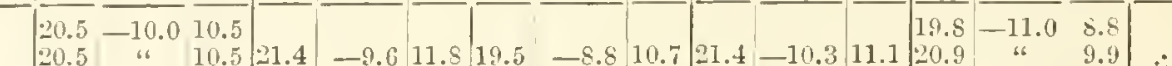

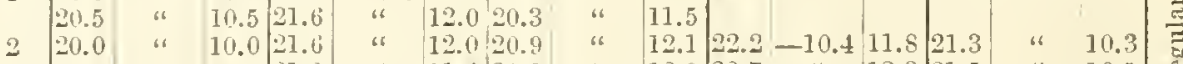

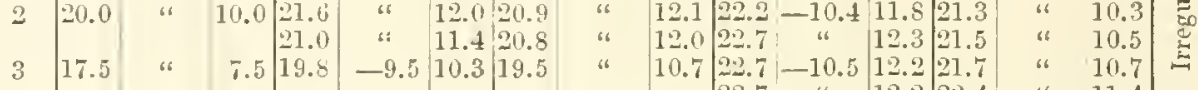

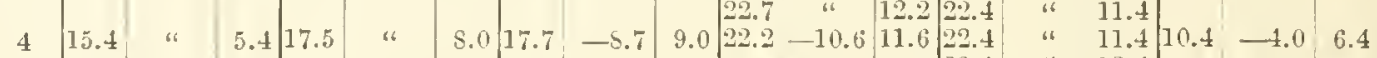

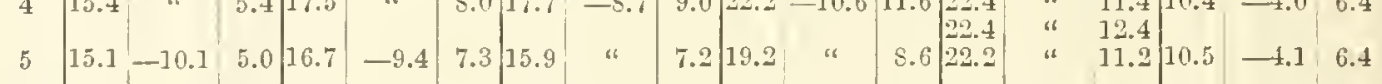

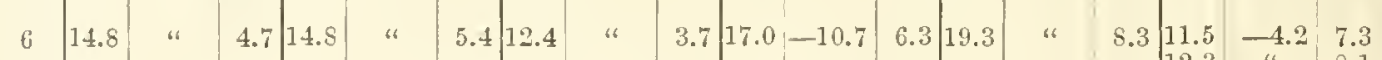

\begin{tabular}{l|l|l|l|l|l|l|l|l|l|l|l|l|l|l|l|l|l|l|}
7 & 14.3 & " & 4.2 & 12.7 & " & 3.3 & 11.2 & " & 2.5 & 15.0 & " & 4.3 & 19.0 & " & 8.0 & 13.2 & " & 8.1 \\
\hline
\end{tabular}

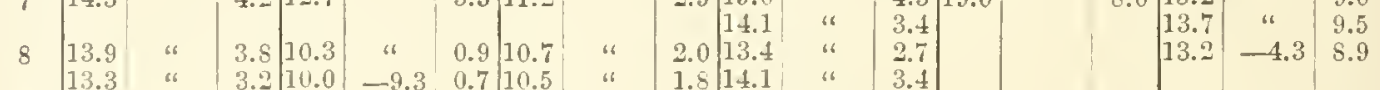

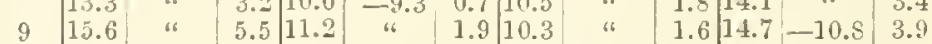

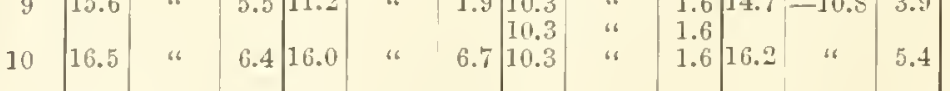

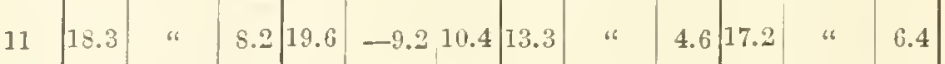

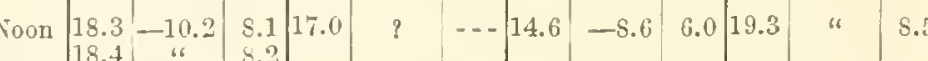

\begin{tabular}{|l|l|l|l|l|l|l|l|l|l|l|l|}
18.4 & " & 8.2 & & & & & & & & & \\
18.6 & " & 8.4 & 19.6 & -9.2 & 10.4 & 16.9 & " & 8.3 & 20.3 & " & 9.5
\end{tabular}

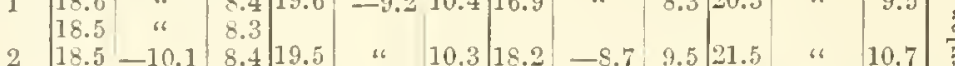

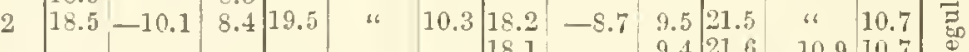

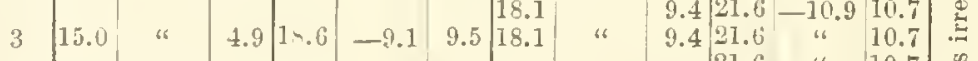

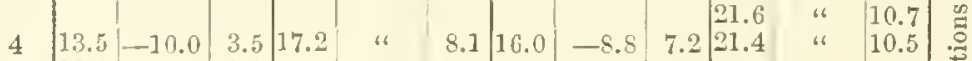

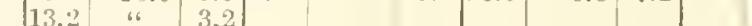

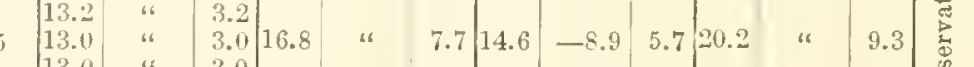

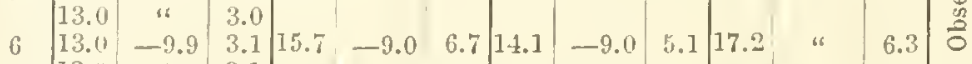

13.0 " 13.1

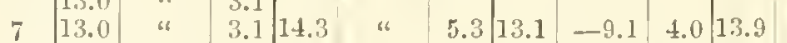

\begin{tabular}{ll|l}
13.0 & " & 3.1 \\
13.0 & 4 & 3.1
\end{tabular}

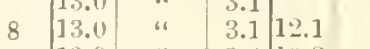

\begin{tabular}{l|l|ll|l|l|l|l|l}
13.3 & 3.4 & 10.2 & " & 1.2 & 12.5 & -9.4 & 3.1 & 13.5
\end{tabular}

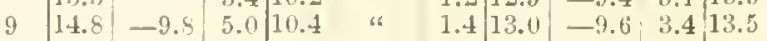

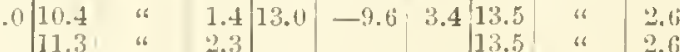

\begin{tabular}{l|l|l|l|l|l|l|l|l|l|l|l|l}
10 & 15.8 & " & 6.0 & 13.2 & -8.9 & 4.3 & 14.2 & -9.8 & 4.4 & 14.0 & " & 3.1
\end{tabular}

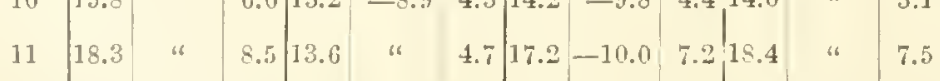

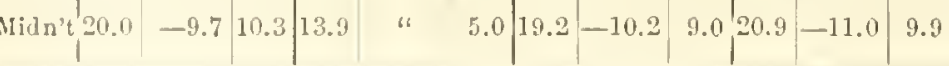

12.6 -4.4 . 5.2

\begin{tabular}{ll|l}
12.0 & -4.5 & 7.5
\end{tabular}

$\begin{array}{llll}11.0 & -4.7 & 6.3\end{array}$

\begin{tabular}{l|l|l}
11.2 & -4.8 & 6.4
\end{tabular}

$11.1 \quad-5.0 \quad 6.1$

\begin{tabular}{|l|l|l|l|l|}
\hline 11.1 & " & 6.1
\end{tabular}

$10.4-5.1 \quad 5.3$

\begin{tabular}{ll|l}
10.5 & "6 & 5.4
\end{tabular}

\begin{tabular}{|l|l|l|}
11.1 & -5.2 & 5.9
\end{tabular}

\begin{tabular}{|l|l|l}
11.8 & -5.4 & 6.4
\end{tabular}

\begin{tabular}{|l|l|l|}
12.2 & -5.5 & 6.7
\end{tabular}

$13.4-5.6 \quad 7.8$

\begin{tabular}{l}
$13.9-5.7$ \\
\hline
\end{tabular}

\begin{tabular}{l|l|l}
14.2 & -5.5 & 8.4
\end{tabular}

$14.3 \quad$ " 14.5

14.0 " 1.2

\begin{tabular}{|l|l|l}
\hline 13.5 & -5.9 & 7.9
\end{tabular}

\begin{tabular}{ll|l}
12.2 & क & 6.3
\end{tabular}

$\begin{array}{lll}12.0 & -6.0 & 6.0\end{array}$

Fect. Incll.

March Ib. Sounding at noon 8 -

$$
\begin{aligned}
& \begin{array}{llllll}
17 . & \text { " } & 7 & 5 & 6 \\
18 . & \text { " } & 7 & 3 & 0
\end{array} \\
& \text { 19. No souuding. } \\
& \text { 20. " " } \\
& \text { 21. Sounding at noon } 6 \quad 1 \quad 0 \\
& 2: 2 \text { " " } 6100 \\
& \text { 23. No sounding. }
\end{aligned}
$$


Series Il.-Thing, Orservations from Januak 28 to APrut 7, 1854.

Ilourly observations on the pulley-gange. Adopted reading of mean level 7.0, expressed in units of the scale. Inereasing numbers inclieate rise of water.

\section{March, 1854}

\begin{tabular}{|c|c|c|c|c|c|c|c|c|c|c|c|c|c|c|c|c|c|c|}
\hline $\begin{array}{l}\text { Jean } \\
\text { snlar } \\
\text { hour. }\end{array}$ & 24 th. & $\begin{array}{c}\text { Reul. } \\
\text { to } \\
\text { level. }\end{array}$ & $\begin{array}{l}\text { Ref. } \\
\text { obs. }\end{array}$ & 25 th. & $\begin{array}{c}\text { Red. } \\
\text { to } \\
\text { level. }\end{array}$ & $\begin{array}{l}\text { Ref. } \\
\text { obs. }\end{array}$ & $26 \mathrm{th}$. & $\begin{array}{c}\text { Rerd. } \\
\text { to } \\
\text { level. }\end{array}$ & $\begin{array}{l}\text { Ref. } \\
\text { obs. }\end{array}$ & $2 \pi$ tb. & $\begin{array}{c}\text { Rerl. } \\
\text { to } \\
\text { level. }\end{array}$ & $\begin{array}{l}\text { Ref. } \\
\text { obs. }\end{array}$ & 2Sth. & $\begin{array}{c}\text { Red. } \\
\text { to } \\
\text { level. }\end{array}$ & $\begin{array}{l}\text { Ref. } \\
\text { obs. }\end{array}$ & $\left.\right|_{1} ^{29 t b .}$ & $\begin{array}{c}\text { Red. } \\
\text { to } \\
\text { level. }\end{array}$ & $\begin{array}{l}\text { Ref. } \\
\text { obs. }\end{array}$ \\
\hline \multirow[b]{2}{*}{1} & & & & & & & & & & & & & & & & $15.5-$ & -3.5 & 12.0 \\
\hline & $\left|\begin{array}{l}11.3 \\
10.9\end{array}\right|$ & -6.0 & $\begin{array}{l}5.3 \\
4.6\end{array}$ & 8.5 & -0.1 & 8.4 & 9.9 & -2.9 & $7.0 \mathrm{l}$ & 10.2 & -1.1 & $9.1 \mid 1$ & 15.0 & -4.7 & 10.3 & $\mid \begin{array}{l}15.3 \\
14.0\end{array}-$ & -3.3 & $\begin{array}{l}12.0 \\
10.7\end{array}$ \\
\hline \multirow[t]{2}{*}{2} & 10.8 & -6.1 & 4.8 & 6.5 & -0.2 & 6.3 & 8.3 & -3.0 & 5.3 & 8.2 & -1.3 & 6.9 & 12.4 & -4.8 & 7.51 & 13.3 & -3.2 & 10.1 \\
\hline & 10.9 & " & 4.8 & 5.5 & " & 5.3 & & & & & & & & & & & & \\
\hline 3 & 11.2 & -6.2 & 5.0 & $\begin{array}{l}5.5 \\
5.5\end{array}$ & -0.3 & 5.2 & $\begin{array}{l}7.5 \\
7.3\end{array}$ & $\begin{array}{l}-3.2 \\
-3.3\end{array}$ & $\begin{array}{r}4.3 \\
4.0\end{array}$ & 6.0 & -1.4 & 4.6 & 9.7 & -5.1 & 4.61 & 11.3 & -3.1 & 8.2 \\
\hline \multirow[t]{2}{*}{4} & 12.1 & " & 5.9 & 5.5 & -0.4 & 5.1 & 7.3 & -3.4 & 3.9 & 4.1 & -1.5 & 2.61 & 9.0 & -5.2 & 3.8 & $9.0-$ & -3.0 & 6.0 \\
\hline & & & & 5.15 & " & 5.2 & 7.3 & -3.5 & 3.8 & 3.9 & -1.6 & 3.3 & & & & & & \\
\hline 5 & $\mid 12.9$ & -6.3 & 6.6 & 5.7 & -0.5 & 5.2 & 7.6 & -3.6 & 4.0 & 3.9 & -1.7 & 2.2 & 8.11 & -5.3 & 2.7 & 7.5 & " & 4.5 \\
\hline \multirow[t]{2}{*}{6} & 13.8 & " & 7.5 & 8.2 & -0.7 & 7.5 & 9.2 & -3.8 & 5.4 & 4.5 & -1.9 & 2.6 & 7.5 & -5.5 & 2.0 & 6.5 & " & 3.5 \\
\hline & & & & & & & & & & & & & 8.22 & -5.6 & 2.6 & 6.5 & " & 3.5 \\
\hline 7 & $\mid \begin{array}{l}15.0 \\
16.0\end{array}$ & -6.4 & 8.6 & 9.2 & -0.8 & $8.4[1$ & 11.8 & -4.0 & 7.8 & 5.3 & -2.0 & 3.3 & 8.7 & -5.7 & 3.0 & $\begin{array}{l}6.5 \\
7.2 \\
7.2\end{array}$ & “" & 3.5 \\
\hline \multirow{2}{*}{8} & $\begin{array}{l}10.0 \\
16.7\end{array}$ & -6.5 & 10.2 & 9.9 & -1.0 & $8.9[1$ & 13.5 & -4.2 & 9.3 & 8.0 & -2.1 & $5.9 \mid 1$ & 11.2 & -5.8 & 5.4 & 8.6 & " & 5.6 \\
\hline & 16.4 & " & $\mid \begin{array}{l}9.9 \\
\mid\end{array}$ & & & & & & & & & & & $\$ 8$ & $10 \therefore:$ & & "6 & $0 \mathrm{~s}$ \\
\hline 9 & 16.0 & -6.6 & 9.4 & $\begin{array}{l}11.2 \\
11.5\end{array}$ & -1.1 & \begin{tabular}{l|l}
10.1 & 1 \\
111.3 & 1
\end{tabular} & $\begin{array}{l}16.0 \\
16.2\end{array}$ & $\begin{array}{l}-4.41 \\
-4.511\end{array}$ & $|11.6|^{1}$ & 12.1 & -2.2 & $\left.9.9\right|^{1}$ & 16.4 & "6 & 10.6 & 12.8 & " & 9.5 \\
\hline \multirow[t]{2}{*}{10} & 15.5 & -6.7 & 8.81 & 11.5 & -3.3 & 10.21 & 16.2 & -4.7 & 11.51 & 13.7 & -2.41 & 11.3 & 16.9 & "“ & 11.1 & 14.9 & " & 11.9 \\
\hline & & & & 11.5 & " & $10.2 \mid 1$ & 15.5 & -4.91 & \begin{tabular}{|l|l|}
10.6 & 1
\end{tabular} & 14.0 & -2.51 & 11.5 & 17.2 & " & 11.4 & & & \\
\hline 11 & 15.0 & -6.8 & 8.2 & 11.5 & -1.4 & $1 \cap .11$ & 14.0 & -5.2 & 8.81 & 14.0 & -2.11 & 11.9 & 17.7 & "، & 11.9 & 16.5 & “" & 13.5 \\
\hline Noon & 8.7 & -0.7 & 8.0 & 9.5 & -1.5 & 8.0 & 12.0 & -5.5 & 6.51 & 13.9 & -2.81 & 11.1 & 16.0 & -5.9 & 10.1 & 17.2 & -3.01 & 14.2 \\
\hline 1 & 7.7 & -0.5 & 7.2 & $8.7-$ & -1.6 & 7.11 & 10.3 & ? & $\cdots 1$ & 12.6 & -2.9 & $9.7 \mid 1$ & 16.3 & " & 10.4 & 17.5 & $"$ & 14.5 \\
\hline \multirow{2}{*}{2} & $5 . s$ & -0.3 & 5.5 & 77 & -1.7 & 6.01 & 8.7 & " & $\ldots$ & 0.4 & -3.1 & $6.3 \mid 1$ & 16.3 & -5.8 & 10.5 & $\begin{array}{l}17.4 \\
16.9\end{array}$ & " & $\begin{array}{l}14.4 \\
13.9\end{array}$ \\
\hline & 5.6 & 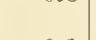 & 5.3 & ... & .1.. & & & & & & & & & & & & & \\
\hline 3 & $\begin{array}{l}5.6 \\
5.6\end{array}$ & -0.1 & $\begin{array}{l}5.5 \\
5.5\end{array}$ & 6.6 & -1.9 & 4.7 & 6.2 & " & - - & 6.6 & -3.2 & $3.4: 1$ & 16.6 & -5.6 & 11.0 & 11.0 & " & 8.0 \\
\hline 4 & 5.6 & 0.0 & 5.6 & $6.3-$ & -2.0 & 4.3 & 3.7 & 0.0 & 3.7 & 6.5 & -3.4 & 3.1 & 12.8 & -5.3 & 7.5 & 7.7 & " & 4.7 \\
\hline 5 & 6.0 & " & 6.0 & $\begin{array}{l}6.6 \\
7.3\end{array}$ & -2.1 & 4.5 & 3.5 & -0.1 & $\begin{array}{l}3.4 \\
3.4\end{array}$ & $\begin{array}{l}5.1 \\
4.9\end{array}$ & $\begin{array}{l}-3.5 \\
-3.6\end{array}$ & $\begin{array}{l}1.6 \\
1.3\end{array}$ & 11.5 & -5.1 & 6.4 & 5.0 & “" & 2.0 \\
\hline 6 & 6.8 & "6 & 6.8 & 7.8 & -2.2 & 5.6 & 3.9 & -0.3 & 3.6 & 5.2 & -3.7 & 1.5 & $\begin{array}{r}10.2 \\
3.5\end{array}$ & -4.9 & 5.3 & $\begin{array}{l}2.9 \\
2.8\end{array}$ & "، & -0.1 \\
\hline 7 & 7.6 & " & 7.6 & 9.3 & -2.3 & 7.0 & 4.6 & -0.4 & 4.2 & 6.9 & -3.9 & 3.0 & 3.8 & -4.7 & -0.9 & 3.2 & " & 0.2 \\
\hline 8 & 7.7 & “ & $7.7 \mid 1$ & $10.9-$ & -2.4 & 8.5 & 6.9 & -0.5 & 6.4 & 8.6 & -4.1 & 4.5 & 6.2 & -4.6 & 1.6 & 5.2 & “ & 3.2 \\
\hline 9 & 8.3 & " & 8.31 & $\left|\begin{array}{l}11.4 \\
1200\end{array}\right|$ & -2.5 & $\begin{array}{r}8.9 \\
9.5\end{array}$ & 9.9 & -0.6 & 9.31 & 12.3 & -4.2 & 8.1 & 11.6 & -4.4 & 7.2 & 7.2 & “ & 4.2 \\
\hline 10 & 9.1 & " & 9.11 & 12.2 & -2.6 & 9.61 & 11.2 & -0.8 & 10.4 & 13.9 & -4.3 & $\mid 9.6$ & 12.6 & -4.2 & 8.4 & 10.5 & " & 7.5 \\
\hline & 9.2 & " & 9.21 & 12.0 & & 9.4 & & & & 14.4 & " 1 & 10.1 & & & & & & \\
\hline 11 & 9.2 & " & 9.21 & 12.0 & -2.7 & $9.3 \mid 1$ & 11.9 & -0.9 & 11.0 & 14.8 & -4.41 & 10.4 & 14.6 & -4.0 & 10.6 & 13.7 & “ & 10.7 \\
\hline Midn't & $\begin{array}{l}8.7 \\
8.4\end{array}$ & “" & $\begin{array}{l}8.7 \\
5.4 .\end{array}$ & 11.5 & -2.8 & & $\begin{array}{l}12.3 \\
11.4\end{array}$ & -1.0 & $\left.\begin{array}{l}11.4 \\
10.4\end{array}\right]$ & $\begin{array}{l}15.3 \\
15.2\end{array}$ & -4.5 & $\begin{array}{l}10.9 \\
10.7\end{array}$ & 15.5 & -3.7 & 11.8 & 15.2 & “ & 12.2 \\
\hline 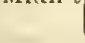 & & & 8.41 & 11.0 & -2.0 & $0.1 p_{1}$ & 11.04 & -1.0 & $10.4]$ & 10.2 & -4.0 & 10.7 & & -0.1 & 11.01 & & & \\
\hline & & & & & Futh. & Fint. & $\mathrm{fl}_{\mathrm{BCh}}$ & & & & & & & & & & & \\
\hline IIa & arch & 4. S'oun & nding : & at noon & 6 & 2 & 6 & & & & & & & & & & & \\
\hline & " 2 & 5. & " & $"$ & (i) & 3 & 6 & & & & & & & & & & & \\
\hline & “" & i. & “" & “" & 7 & 11 & (1 & & & & & & & & & & & \\
\hline & $" 6$ & 7. No so & sountis & ing. & & & & & & & & & & & & & & \\
\hline & " & ১. Soun & meling : & at $n 00 n$ & 7 & 4 & ") & Corre & rection & 1113 & sountin & $n g$ - & $.6, \mathrm{by}$ & 8 сыто & -5.2, & \&, mean & $11-5.8$ & \\
\hline & .6 & 9. & “" & “ & 7 & 5 & i & & & & & & & & & & & \\
\hline
\end{tabular}


Series II.-Tidal Observations from Jaxuary 28 to A pril 7, 1854.

Hourly observations on the pulley-gange. Atopted reading of mean level 7.0, expressed in anits of the scale. Increasing numbers indicate rise of water.

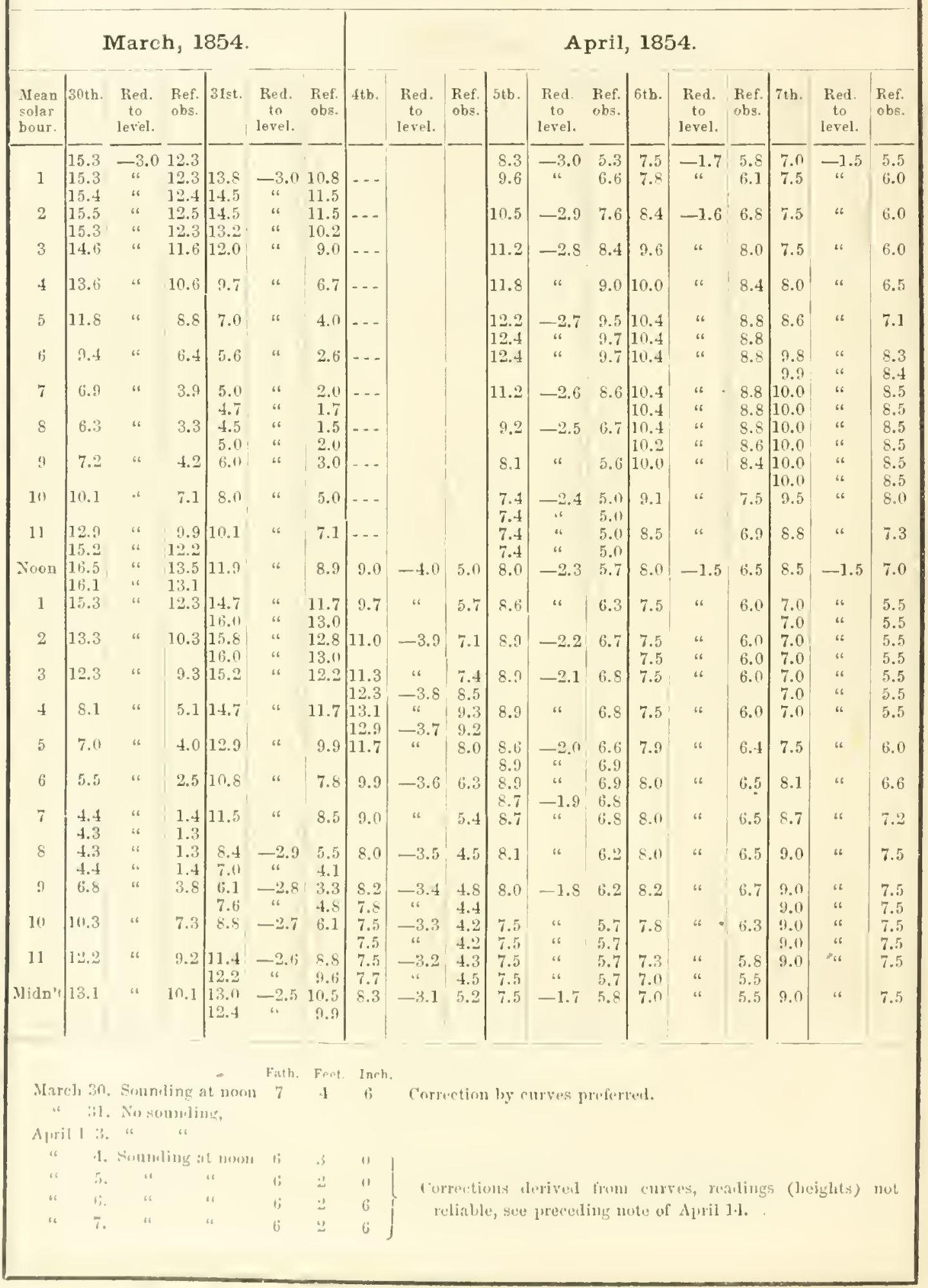


Series III.-'Tidal Observations fron A pril 20 to A vaugt 3, 1854.

Homly observations on the pulley-gauge. Adopted reading of mean level 7.0, expressed in units of the seale. Inereasing numbers indicate rise of water.

\section{April, 1854.}

\begin{tabular}{|c|c|c|c|c|c|c|c|c|c|c|c|c|c|c|c|c|c|c|}
\hline $\begin{array}{l}\text { Mean } \\
\text { sular } \\
\text { lour. }\end{array}$ & $20 \mathrm{tb}$. & $\begin{array}{c}\text { Red. } \\
\text { to } \\
\text { level. }\end{array}$ & $\begin{array}{l}\text { Ref. } \\
\text { obs. }\end{array}$ & $21 \mathrm{st}$. & $\begin{array}{c}\text { Red. } \\
\text { to } \\
\text { level. }\end{array}$ & $\begin{array}{l}\text { Ref. } \\
\text { obs. }\end{array}$ & 221. & $\begin{array}{c}\text { Red. } \\
\text { to } \\
\text { level. }\end{array}$ & $\begin{array}{l}\text { Ref. } \\
\text { obs. }\end{array}$ & $23 \mathrm{~d}$. & $\begin{array}{c}\text { Red. } \\
\text { to } \\
\text { level. }\end{array}$ & $\begin{array}{l}\text { Ref. } \\
\text { obs. }\end{array}$ & 24 th. & $\begin{array}{c}\text { Red. } \\
\text { to } \\
\text { level. }\end{array}$ & $\begin{array}{l}\text { Ref. } \\
\text { obs. }\end{array}$ & $25+11$. & $\begin{array}{c}\text { Red. } \\
\text { to } \\
\text { level. }\end{array}$ & $\begin{array}{l}\text { Ref. } \\
\text { obs. }\end{array}$ \\
\hline 1 & & & & $\begin{array}{l}6.7 \\
7.3\end{array}$ & -2.2 & $\begin{array}{l}4.5 \\
5.1\end{array}$ & $\begin{array}{l}7.8 \\
7.5 \\
7.5\end{array}$ & $\begin{array}{c}-3.5 \\
6 \\
4\end{array}$ & $\begin{array}{l}4.3 \\
4.0 \\
4.0\end{array}$ & 10.0 & -4.2 & 5.8 & 9.4 & -4.0 & 5.4 & 11.1 & -3.5 & 7.6 , \\
\hline 2 & & & & 7.9 & -2.3 & 5.6 & 7.7 & -3.6 & 4.1 & $\begin{array}{l}9.9 \\
8.5\end{array}$ & " & $\begin{array}{l}5.7 \\
4.3\end{array}$ & 7.5 & " & 3.5 & 8.6 & 16 & 5.3 \\
\hline 3 & & & & 9.0 & -2.4 & 6.6 & 7.9 & 16 & 4.3 & $\begin{array}{l}8.3 \\
8.5\end{array}$ & " " & $\begin{array}{l}4.1 \\
4.3\end{array}$ & $\begin{array}{l}6.7 \\
6.5\end{array}$ & "6 & $\begin{array}{l}2.7 \\
2.5\end{array}$ & 7.5 & " & 4.0 \\
\hline 4 & 䒕 & & & 10.2 & ": & 7.8 & 8.9 & -3.7 & 5.2 & 8.7 & $"$ & 4.5 & $\begin{array}{l}6.5 \\
6.7\end{array}$ & -3.9 & $\begin{array}{l}2.6 \\
2.6\end{array}$ & $\begin{array}{l}6.4 \\
5.8\end{array}$ & -3.4 & $\begin{array}{l}3.0 \\
2.4\end{array}$ \\
\hline 5 & 变 & & & 11.4 & -2.5 & 8.9 & 9.7 & " & 6.0 & 10.2 & $"$ & 6.0 & 7.1 & "6 & 3.2 & $\begin{array}{l}5.11 \\
6.0\end{array}$ & 46 & $\begin{array}{l}1.6 \\
2.6\end{array}$ \\
\hline 6 & & & & 12.1 & " & 9.6 & 12.2 & -3.8 & 8.4 & 11.8 & " & 7.6 & 8.8 & " & 4.9 & 7.0 & " & 3.6 \\
\hline 7 & & & & $\begin{array}{l}12.5 \\
12.6\end{array}$ & -2.6 & $\begin{array}{r}9.9 \\
10.0\end{array}$ & $\begin{array}{l}13.3 \\
13.7\end{array}$ & $"$ & $\begin{array}{l}9.5 \\
9.9\end{array}$ & $\begin{array}{l}13.2 \\
14.0\end{array}$ & "6 & $\begin{array}{l}9.0 \\
9.8\end{array}$ & 10.5 & " & 6.6 & 8.5 & " & 5.1 \\
\hline 8 & & & & 12.5 & -2.7 & 9.8 & $\begin{array}{l}14.3 \\
14.0\end{array}$ & -3.9 & $\begin{array}{l}10.4 \\
10.1\end{array}$ & $\begin{array}{l}14.9 \\
14.9\end{array}$ & 16 & $\begin{array}{l}10.7 \\
10.7\end{array}$ & 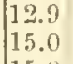 & $"$ & $\begin{array}{r}9.0 \\
11.1\end{array}$ & 10.9 & -3.3 & 7.6 \\
\hline 9 & 11.3 & -1.0 & 10.3 & 11.0 & -2.8 & 8.2 & 13.7 & $" 6$ & 9.8 & 13.0 & "6 & 8.8 & 15.0 & $"$ & 11.1 & $\begin{array}{l}13.4 \\
14.5\end{array}$ & "6 & $\begin{array}{l}10.1 \\
11.2\end{array}$ \\
\hline 10 & 8.3 & -1.1 & 7.2 & 9.9 & " & 7.1 & 12.3 & " & 8.4 & ?8.1 & $?$ & $\cdots$ & 14.9 & " & 11.0 & $\begin{array}{l}15.5 \\
15.3\end{array}$ & " & $\begin{array}{l}12.2 \\
12.0\end{array}$ \\
\hline 11 & $\begin{array}{l}6.7 \\
6.4\end{array}$ & -1.2 & $\begin{array}{l}5.5 \\
5.2\end{array}$ & 8.5 & -2.9 & 5.6 & 11.3 & $"$ & 7.4 & 10.7 & "4 & -- & 14.6 & " & 10.7 & 14.1 & "6 & 10.8 \\
\hline Noon & $\begin{array}{l}6.4 \\
6.4\end{array}$ & -1.3 & $\begin{array}{l}5.1 \\
5.1\end{array}$ & $\begin{array}{l}7.9 \\
7.4\end{array}$ & -3.0 & $\begin{array}{l}4.9 \\
4.4\end{array}$ & 10.7 & -4.0 & 6.7 & 11.5 & -1.3 & 7.2 & 12.9 & -3.8 & n.1 & 12.5 & -3.2 & 9.3 \\
\hline 1 & 6.7 & " & 5.4 & $\begin{array}{l}7.0 \\
7.0\end{array}$ & $"$ " & $\begin{array}{l}4.0 \\
4.0\end{array}$ & 8.7 & $"$ & 4.7 & 9.5 & $" 1$ & 5.2 & 10.6 & " & 6.8 & 111.3 & " & 7.1 \\
\hline 2 & 7.1 & -1.4 & 5.7 & $\begin{array}{l}7.0 \\
7.4\end{array}$ & -3.1 & $\begin{array}{l}3.9 \\
4.3\end{array}$ & $\begin{array}{l}8.3 \\
8.11\end{array}$ & 6 & $\begin{array}{l}4.3 \\
4.0\end{array}$ & 8.3 & " & 4.0 & 9.0 & $"$ & 5.2 & 5.3 & " & 5.1 \\
\hline 3 & 7.9 & " & 6.5 & 8.0 & "I & 4.9 & $\begin{array}{l}8.0 \\
8.0\end{array}$ & " & $\begin{array}{l}4.0 \\
4.0\end{array}$ & $\begin{array}{l}7.2 \\
6.6\end{array}$ & -4.2 & $\begin{array}{l}3.0 \\
2.4\end{array}$ & 7.4 & " & 3.6 & 6.9 & " & 3.7 \\
\hline 4 & 9.0 & -1.5 & 7.5 & 8.8 & -3.2 & 5.6 & $\begin{array}{l}8.0 \\
8.3\end{array}$ & $" 6$ & $\begin{array}{l}4.0 \\
4.3\end{array}$ & $\begin{array}{l}6.1 \\
6.0\end{array}$ & " & $\begin{array}{l}1.9 \\
1.8\end{array}$ & $\begin{array}{l}6.1 \\
5.4\end{array}$ & -3.7 & $\begin{array}{l}2.4 \\
1.7\end{array}$ & $\begin{array}{l}5.2 \\
5.0\end{array}$ & 16 & $\begin{array}{l}2.0 \\
1.8\end{array}$ \\
\hline 5 & $\begin{array}{l}9.5 \\
9.7\end{array}$ & " & $\begin{array}{l}8.0 \\
8.2\end{array}$ & 8.5 & 66 & 5.3 & 9.0 & -4.1 & 4.9 & 6.5 & -4.1 & 2.4 & 5.7 & " & 2.0 & $\begin{array}{l}5.0 \\
5.0\end{array}$ & " & $\begin{array}{l}1.5 \\
1.8\end{array}$ \\
\hline 6 & $\begin{array}{l}9.7 \\
9.7\end{array}$ & -1.6 & $\begin{array}{l}8.1 \\
8.1\end{array}$ & $\begin{array}{r}9.2 \\
10.1\end{array}$ & $\begin{array}{l}-3.3 \\
6\end{array}$ & $\begin{array}{l}5.9 \\
6.8\end{array}$ & 10.8 & $" 6$ & 6.7 & 7.5 & " & 3.4 & 8.1 & " & 4.4 & 5.2 & $" 6$ & 2.0 \\
\hline 7 & 9.5 & " & 7.9 & $\begin{array}{l}10.5 \\
10.5\end{array}$ & "6 & $\begin{array}{l}7.2 \\
7.2\end{array}$ & 11.5 & $" 6$ & 7.4 & 9.3 & " & 5.2 & $9.1)$ & "6 & 5.3 & 7.5 & " & 4.3 \\
\hline 8 & 8.8 & -1.7 & 7.1 & $\begin{array}{l}10.5 \\
10.5\end{array}$ & "6 & 7.2 & 12.5 & " & 8.4 & 10.5 & " & 6.7 & 10.4 & -3.6 & 6.8 & 9.7 & -3.1 & $6.6^{\circ}$ \\
\hline 9 & 7.9 & -1.8 & 6.1 & $\begin{array}{l}10.5 \\
10.0\end{array}$ & -3.4 & $\begin{array}{l}7.1 \\
6.6\end{array}$ & $\begin{array}{l}13.0 \\
13.9\end{array}$ & " & $\begin{array}{l}8.9 \\
9.8\end{array}$ & $\begin{array}{l}12.0 \\
12.8\end{array}$ & "6 & $\begin{array}{l}7.9 \\
8.7\end{array}$ & $\begin{array}{l}13.5 \\
13.5\end{array}$ & $" 6$ & $\begin{array}{l}9.9 \\
9.9\end{array}$ & $\ldots$ & & $\ldots$ \\
\hline 10 & 7.0 & -1.9 & 5.1 & 9.1 & "6 & 5.7 & $\begin{array}{l}13.9 \\
13.0\end{array}$ & $\begin{array}{c}\text { " } \\
-4.2\end{array}$ & $\begin{array}{l}9.8 \\
8.8\end{array}$ & $\begin{array}{l}13.6 \\
13.6\end{array}$ & -4.0 & $\begin{array}{l}9.6 \\
9.6\end{array}$ & $\begin{array}{l}13.5 \\
12.5\end{array}$ & " & $\begin{array}{r}9.9 \\
\therefore .9\end{array}$ & $\cdots$ & & $\cdots$ \\
\hline 11 & 6.9 & -2.0 & 4.9 & 8.4 & "6 & 5.0 & 12.0 & " & 7.8 & 13.0 & " & 9.0 & 12.0 & " & 8.4 & $\mid \cdots$ & & $\cdots$ \\
\hline Midn't & 6.9 & -2.1 & 4.5 & 7.9 & -3.5 & 4.4 & 11.3 & " & 7.1 & 9.0 & $"$ & 5.11 & 11.5 & -3.5 & 8.0 & $\ldots$ & & $\cdots$ \\
\hline
\end{tabular}

April 20. No sounding.

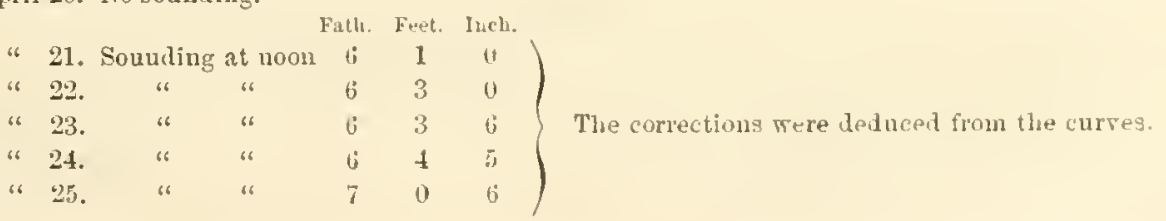


Series III.-Tidal Obeertations from A pril 20 to August 3, 1854.

Hoully ohscrrations on the pulley-gauge. Adopted reading of mean level 7.0, expressed in mits of the seale. Increasing numbers indieate rise of water.

\begin{tabular}{|c|c|c|c|c|c|c|c|c|c|c|c|c|c|c|c|c|c|c|}
\hline \multicolumn{16}{|c|}{ April, 1854.} & \multicolumn{3}{|c|}{ May, 1854} \\
\hline $\begin{array}{l}\text { Mean } \\
\text { solar } \\
\text { hour. }\end{array}$ & $\left.\right|^{26 \text { th. }}$ & $\begin{array}{c}\text { Red. } \\
\text { to } \\
\text { level. }\end{array}$ & $\begin{array}{l}\text { Ref. } \\
\text { obs. }\end{array}$ & $271 \mathrm{~h}$. & $\begin{array}{c}\text { Red. } \\
\text { to } \\
\text { level. }\end{array}$ & $\begin{array}{l}\text { lief. } \\
\text { ous. }\end{array}$ & 28 th. & $\begin{array}{c}\text { Red. } \\
\text { to } \\
\text { level. }\end{array}$ & $\begin{array}{l}\text { Ref. } \\
\text { olss. }\end{array}$ & $29 \mathrm{th}$. & $\begin{array}{c}\text { Red. } \\
\text { to } \\
\text { level. }\end{array}$ & $\begin{array}{l}\text { Ref. } \\
\text { obs. }\end{array}$ & 30 th. & $\begin{array}{c}\text { Red. } \\
\text { to } \\
\text { level. }\end{array}$ & $\begin{array}{l}\text { Tief. } \\
\text { obs. }\end{array}$ & 1st. & $\begin{array}{c}\text { Reil. } \\
\text { to } \\
\text { level. }\end{array}$ & $\begin{array}{l}\text { Ref. } \\
\text { obs. }\end{array}$ \\
\hline 1 & $\begin{array}{c}10.1 \\
9.1\end{array}$ & $\begin{array}{c}-3.0 \\
"\end{array}$ & $\begin{array}{l}7.1 \\
6.1\end{array}$ & $\begin{array}{l}14.4 \\
14.5 \\
14.1 \\
12.8\end{array}$ & $\begin{array}{c}" \\
-3.0 \\
" \\
"\end{array}$ & $\begin{array}{r}11.4 \\
11.5 \\
11.1 \\
9.8\end{array}$ & 9.2 & $\begin{array}{c}-3.0 \\
،\end{array}$ & 6.2 & $\mid \begin{array}{l}16.4 \\
16.0 \\
14.9\end{array}$ & -3.4 & $\begin{array}{l}13.0 \\
12.6 \\
11.5\end{array}$ & $\left\{\begin{array}{l}16.4 \\
15.3 \\
12.8\end{array}\right.$ & -3.6 & $\begin{array}{r}12.8 \\
11.7 \\
9.2\end{array}$ & $\begin{array}{l}16.2 \\
16.5 \\
16.5 \\
15.9\end{array}$ & $\begin{array}{c}6 \\
" 6 \\
-4.0\end{array}$ & $\begin{array}{l}12.3 \\
12.6 \\
12.6 \\
11.9\end{array}$ \\
\hline 3 & 7.1 & "، & $\begin{array}{l}4.1 \\
3.3\end{array}$ & 10.5 & 6 & 7.5 & 7.0 & " & 4.0 & 13.1 & "6 & 9.7 & 10.3 & "6 & 6.7 & 14.7 & "، & 10.7 \\
\hline 4 & $\begin{array}{l}5.6 \\
5.6\end{array}$ & $" 6$ & $\begin{array}{l}2.6 \\
2.8\end{array}$ & 8.3 & $" 6$ & 5.3 & $\begin{array}{l}6.0 \\
5.3\end{array}$ & 4 & $\begin{array}{l}3.0 \\
2.3\end{array}$ & 11.7 & "6 & 8.3 & 7.6 & 4 & 4.0 & 13.4 & 46 & 9.4 \\
\hline 5 & 6.3 & 36 & 3.3 & 6.8 & 6 & 3.8 & $\begin{array}{l}5.0 \\
5.1\end{array}$ & $" 6$ & $\begin{array}{l}2.0 \\
2.1\end{array}$ & 9.8 & 16 & 6.4 & $\begin{array}{l}6.2 \\
5.5\end{array}$ & "6 & $\begin{array}{l}2.6 \\
1.9\end{array}$ & 12.2 & 6 & 8.2 \\
\hline 6 & 7.3 & $" 6$ & 4.3 & $\begin{array}{l}4.7 \\
4.0\end{array}$ & 46 & $\begin{array}{l}1.7 \\
1.0\end{array}$ & 5.7 & 16 & 2.7 & 8.0 & 4 & 4.6 & 6.2 & 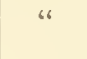 & 2.6 & 10.0 & 6 & 6.0 \\
\hline 7 & 8.5 & 66 & 5.5 & $\begin{array}{l}4.0 \\
5.9\end{array}$ & "6 & $\begin{array}{l}1.11 \\
2.9\end{array}$ & 6.6 & -3.1 & 3.5 & $\begin{array}{l}5.9 \\
5.5\end{array}$ & "6 & $\begin{array}{l}2.5 \\
2.1\end{array}$ & 7.7 & 4 & 4.1 & 8.2 & $"$ & 4.9 \\
\hline 8 & 9.4 & " & 6.4 & 8.7 & 4 & 5.7 & 7.5 & 16 & 4.4 & i.:2 & tt & 2.8 & 9.1 & $"$ & 5.5 & $\begin{array}{l}8.2 \\
8.9\end{array}$ & 46 & $\begin{array}{l}4.2 \\
4.0\end{array}$ \\
\hline 9 & 11.6 & " & 7.6 & 111.9 & " & 7.9 & 8.4 & $" 6$ & 5.7 & 8.3 & "6 & 4.9 & 10.1 & 16 & 6.5 & $\begin{array}{l}7.9 \\
8.4\end{array}$ & 6 & $\begin{array}{l}3.9 \\
4.1\end{array}$ \\
\hline 10 & 11.4 & 16 & $\therefore 4$ & 12.7 & 4 & 9.7 & 10.3 & $" 6$ & 7.2 & 10.9 & "s & 7.5 & 11.2 & 46 & 7.6 & 9.7 & "6 & 5.7 \\
\hline 11 & $\begin{array}{l}13.9 \\
14.4\end{array}$ & 4 & $\begin{array}{l}10.9 \\
11.4\end{array}$ & $\begin{array}{l}14.4 \\
14.5\end{array}$ & 46 & $\left|\begin{array}{l}11.4 \\
11.5\end{array}\right|$ & 11.7 & " & 8.6 & $\begin{array}{l}13.5 \\
14.6\end{array}$ & " & $\begin{array}{l}10.1 \\
11.2\end{array}$ & 13.0 & 46 & 3.4 & 11.9 & "s & 7.9 \\
\hline Noon & $\begin{array}{l}15.0 \\
14.11\end{array}$ & " " & $\begin{array}{l}12.0 \\
11.0\end{array}$ & 15.1 & $4:$ & $\begin{array}{l}1 \because .1 \\
12.2\end{array}$ & $\begin{array}{l}13.7 \\
14.5\end{array}$ & -3.2 & $\begin{array}{l}10.5 \\
11.3\end{array}$ & $\begin{array}{l}15.5 \\
1+.5\end{array}$ & -3.5 & $\begin{array}{l}12.0 \\
11.0\end{array}$ & $\begin{array}{l}14.7 \\
15.0\end{array}$ & -3.7 & $\begin{array}{l}11.0 \\
11.3\end{array}$ & $\begin{array}{l}13.1 \\
13.5\end{array}$ & -4.1 & $\begin{array}{l}9.0 \\
9.4\end{array}$ \\
\hline 1 & 13.1 & $" 6$ & 11.1 & 15.0 & " & 12.0 & $\begin{array}{l}14.8 \\
14.4\end{array}$ & "6 & $\begin{array}{l}11.6 \\
11.2\end{array}$ & 13.2 & " & 9.7 & 14.4 & " & 10.7 & 13.0 & 6 & 8.9 \\
\hline 2 & 111.7 & $" 4$ & 7.7 & 12.4 & " & 9.4 & 14.3 & 6 & 11.1 & 10.9 & $"$ " & 7.4 & 12.6 & 66 & 8.9 & 11.3 & 6 & 7.2 \\
\hline 3 & 5.7 & $" 6$ & 5.7 & 10.3 & "6 & 7.3 & 12.4 & $" 6$ & 0.2 & 9.6 & $" 6$ & $(i .1$ & 111.2 & 6. & 6.5 & 10.2 & -4.2 & 6.0 \\
\hline 4 & 6.1 & $"$ & 3.1 & $\begin{array}{l}7.3 \\
6.2\end{array}$ & $" 6$ & $\begin{array}{l}4.3 \\
3.2\end{array}$ & 9.2 & 6 & 6.11 & 7.1 & "4 & 3.6 & $\begin{array}{l}8.1 \\
6.3\end{array}$ & " & $\begin{array}{l}4.3 \\
2.13\end{array}$ & 9.8 & $"$ & 5.6 \\
\hline 5 & 4.1 & " & 1.1 & $\begin{array}{l}5.2 \\
5.9\end{array}$ & 46 & $\begin{array}{l}2.2 \\
2.9\end{array}$ & li. 0 & 66 & 2.8 & $\begin{array}{l}5.3 \\
5.1\end{array}$ & -3.5 & $\begin{array}{l}1.8 \\
1.5\end{array}$ & $\begin{array}{l}5.5 \\
5.5\end{array}$ & -3.8 & $\begin{array}{l}1.7 \\
1.7\end{array}$ & $\begin{array}{l}9.1 \\
\therefore .6\end{array}$ & " & $\begin{array}{l}4.9 \\
4.4\end{array}$ \\
\hline 6 & $\begin{array}{l}3.2 \\
3.1\end{array}$ & $" 4$ & $\begin{array}{l}11.2 \\
11.1\end{array}$ & 7.7 & 6 & 4.7 & $\begin{array}{l}5.5 \\
5.3\end{array}$ & $\begin{array}{l}6 \\
6\end{array}$ & $\begin{array}{l}2.3 \\
2.1\end{array}$ & 5.1 & ". & 1.16 & (j:Z & .. & 2.4 & $\begin{array}{l}8.0 \\
8.0\end{array}$ & 6 & $\begin{array}{l}3.8 \\
3.8\end{array}$ \\
\hline 7 & 4.6 & $" 6$ & 1.6 & 9.6 & 6 & li.ti & $\begin{array}{l}5.3 \\
\text { (i. } 1\end{array}$ & $-3 . .3$ & $\begin{array}{l}2.11 \\
2.8\end{array}$ & 7.1 & $" 6$ & 3.6 & 7.6 & 86 & 3.8 & $\begin{array}{l}5.6 \\
8.4\end{array}$ & -4.3 & $\begin{array}{l}3.7 \\
4.1\end{array}$ \\
\hline 8 & 7.2 & "6 & 4.2 & 111.8 & 6 & 7.8 & 7.8 & "6 & 4.5 & 8.6 & 6 & 5.1 & 8.5 & $\therefore$ & 4.7 & 8.8 & "6 & 4.5 \\
\hline 9 & 10.7 & $" 6$ & 7.7 & 12.3 & " & 9.3 & 10.1 & " & 6.9 & 10.4 & " & Ci.9 & 9.5 & 6. & 5.7 & 10.2 & $"$ & 5.9 \\
\hline 10 & 12.3 & 16 & 9.3 & 1.1 .0 & $" 6$ & 11.0 & 12.9 & " & 9.5 & 13.4 & $" 6$ & 9.9 & 11.3 & 6 & 7.5 & 11.3 & $"$ & 7.0 \\
\hline 11 & 14.2 & " & 11.2 & 15.7 & $?$ & $\ldots$ & 14.8 & "6 & 11.5 & $\begin{array}{l}15.3 \\
16.2\end{array}$ & "6 & 11.8 & 13.9 & -3.9 & 10.0 & 12.7 & " & 8.4 \\
\hline $\operatorname{didn}{ }^{9} t$ & 14.3 & $" 6$ & 11.3 & 16.7 & " & 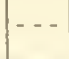 & 15.5 & -3.3 & 12.2 & 16.4 & 4 & 12.9 & 15.4 & 66 & 11.5 & 14.1 & -4.4 & 9.7 \\
\hline
\end{tabular}

lith. Fuet, furlh.

April 621\%. Soumling at noon 7 3 3 () Corrections deduced from the curres.

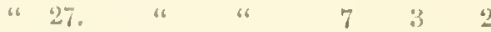

" " " "

" 2w-:30. No souniliug.

May 1. Fomuling at noun 744 
Serifs III.-Tidal Orservations from A pril 20 to A uqust 3, 1854.

Hourly obscrvations on the pulley-gauge. Adopted reading of mean level 7.0, expressed in units of the seale. Inereasing numbers indicate rise of water.

May, 1854.

\begin{tabular}{|c|c|c|c|c|c|c|c|c|c|c|c|c|c|c|c|c|c|c|}
\hline $\begin{array}{l}\text { Mean } \\
\text { solar } \\
\text { hour. }\end{array}$ & $2 \mathrm{u}$. & $\begin{array}{c}\text { Red. } \\
\text { to } \\
\text { level. }\end{array}$ & $\begin{array}{l}\text { Ref. } \\
\text { obs. }\end{array}$ & 34. & $\begin{array}{c}\text { Red. } \\
\text { to } \\
\text { level. }\end{array}$ & $\begin{array}{l}\text { Ref. } \\
\text { obs. }\end{array}$ & 4th. & $\begin{array}{c}\text { Red. } \\
\text { to } \\
\text { lerel. }\end{array}$ & $\begin{array}{l}\text { Ref. } \\
\text { obs. }\end{array}$ & ith. & $\begin{array}{c}\text { Red. } \\
\text { to } \\
\text { level. }\end{array}$ & $\begin{array}{l}\text { Ref. } \\
\text { obs. }\end{array}$ & Sth. & $\begin{array}{c}\text { Red. } \\
\text { to } \\
\text { level. }\end{array}$ & $\begin{array}{l}\text { Pef. } \\
\text { olus. }\end{array}$ & Oth. & $\begin{array}{c}\text { Perd. } \\
\text { to } \\
\text { level. }\end{array}$ & $\begin{array}{l}\text { Ref. } \\
\text { obse. }\end{array}$ \\
\hline & & & & & & & & & & 10.2 & -4.2 & 6.0 & 10.5 & -4.8 & 5.7 & & & \\
\hline 1 & 15.1 & -4.4 & 10.7 & 13.2 & 64 & 8.5 & 13.6 & -5.2 & 8.4 & $\begin{array}{l}10.2 \\
10.5\end{array}$ & "6 & $\begin{array}{l}6.0 \\
6.3\end{array}$ & $\begin{array}{l}10.0 \\
11.5\end{array}$ & " & $\begin{array}{l}5.2 \\
6.7\end{array}$ & $\begin{array}{l}10.7 \\
10.0\end{array}$ & -5.5 & $\begin{array}{l}5.2 \\
4.5\end{array}$ \\
\hline 2 & $\begin{array}{l}16.7 \\
16.8\end{array}$ & "6 & $\begin{array}{l}12.3 \\
12.4\end{array}$ & 14.0 & " & 9.3 & 14.0 & -5.3 & 8.7 & 10.8 & '6 & 6.6 & 11.5 & -4.9 & 6.6 & $\begin{array}{l}10.0 \\
10.5\end{array}$ & -5.6 & $\begin{array}{l}4.4 \\
+.9\end{array}$ \\
\hline 3 & 16.5 & 4 & 12.1 & $\begin{array}{l}14.2 \\
14.3\end{array}$ & 16 & $\begin{array}{l}9.5 \\
9.6\end{array}$ & 14.8 & -5.4 & 9.4 & 11.3 & $"$ & 7.1 & 11.8 & $"$ & 0.9 & 11.3 & 4 & 5.7 \\
\hline 4 & 14.2 & -4.5 & 9.7 & $\begin{array}{l}14.3 \\
14.2\end{array}$ & 66 & $\begin{array}{l}9.6 \\
9.5\end{array}$ & 16.1 & -5.6 & 10.5 & 11.8 & " & 7.6 & 12.4 & 66 & 7.5 & 12.6 & -5.7 & 6.9 \\
\hline 5 & 12.5 & " & 8.0 & 14.1 & $"$ & 9.4 & 16.2 & -5.8 & 10.4 & 12.4 & 6 & 8.2 & 13.0 & " & 8.1 & 14.3 & 6 & 8.6 \\
\hline 6 & 11.0 & 6 & 6.5 & 13.1 & $"$ & 8,4 & $\begin{array}{l}16.2 \\
15.7\end{array}$ & $\begin{array}{l}-6.0 \\
-6.1\end{array}$ & $\begin{array}{r}10.2 \\
9.6\end{array}$ & 13.2 & -4.3 & 8.9 & 14.1 & -5.0 & 9.1 & 15.6 & "6 & 9.9 \\
\hline 7 & 8.4 & 6 & 3.9 & 12.0 & " & 7.3 & 15.3 & -6.3 & 9.0 & 13.2 & " & 8.9 & 16.2 & " & 11.2 & $\begin{array}{l}16.4 \\
16.9\end{array}$ & -5.8 & $\begin{array}{l}10.6 \\
11.1\end{array}$ \\
\hline 8 & $\begin{array}{l}0.7 \\
0.4\end{array}$ & $" 4$ & $\begin{array}{l}2.2 \\
1.8\end{array}$ & $\begin{array}{l}10.9 \\
10.9\end{array}$ & 16 & $\begin{array}{l}6.2 \\
5.5\end{array}$ & & & & 13.0 & " & 8.7 & $\begin{array}{l}16.0 \\
16.5\end{array}$ & "6 & $\begin{array}{l}11.0 \\
11.5\end{array}$ & $\begin{array}{l}17.2 \\
17.2\end{array}$ & -5.9 & $\begin{array}{l}11.3 \\
11.3\end{array}$ \\
\hline 9 & 6.4 & -4.6 & 1.5 & 111.0 & "6 & 5.3 & & & & 12.2 & "6 & 7.9 & $16.5)$ & -5.1 & 11.4 & 17.2 & -6.0 & 11.2 \\
\hline 10 & 7.4 & " & 2.5 & $\begin{array}{l}10.3 \\
10.5 \\
10.8\end{array}$ & $\begin{array}{l}16 \\
16 \\
16\end{array}$ & 5.6 & & & & 11.0 & 66 & 0.7 & $\begin{array}{l}16.3 \\
16.0\end{array}$ & 6 & $\begin{array}{l}11.2 \\
10.9\end{array}$ & $\begin{array}{l}16.6 \\
16.0\end{array}$ & -6.1 & $\begin{array}{r}119.6 \\
9.9\end{array}$ \\
\hline 11 & 9.5 & 66 & 4.8 & 11.0 & 46 & $\begin{array}{l}\text { n.1 } \\
\text { ti.3 }\end{array}$ & & & & 10.2 & " & 5.9 & 15.1 & " & 10.0 & 1.1 .9 & -6.2 & 8.7 \\
\hline Noon & 11.3 & -4.7 & i. 6 & 11.1 & 4.7 & 6.7 & & & & 9.0 & -4.4 & 4.6 & 14.1 & -5.2 & 8.3 & 13.8 & -6.3 & 7.5 \\
\hline 1 & 13.1 & " & 8.4 & 13.8 & 46 & 8.1 & & & & $\begin{array}{l}8.1 \\
8 . n\end{array}$ & $\begin{array}{l}16 \\
16\end{array}$ & 3.7 & 13.0 & $" 6$ & 7.8 & ? & " & .. \\
\hline 2 & 14.9 & $"$ & 10.2 & 13.8 & " & 0.1 & $\ddot{\dot{E}}$ & & & 8.0 & $" 6$ & 3.13 & $10.6^{\circ}$ & 6 & 5.7 & 11.2 & " & 4.9 \\
\hline 3 & 15.0 & $"$ & $\begin{array}{r}10.3 \\
0.8\end{array}$ & $\begin{array}{l}13.8 \\
13.8\end{array}$ & 16 & 9.1 & $\vec{z}$ & & & 8.0 & 66 & 3.16 & 90 & -5.3 & 3.7 & 10.0 & -6.2 & 3.5 \\
\hline 3 & 14.0 & 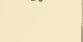 & $1 . n$ & $\begin{array}{l}13.8 \\
13.8\end{array}$ & "6 & 9.1 & $\Xi$ & & & 9.4 & 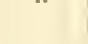 & 4.1 & 8.4 & 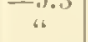 & 3.1 & $\begin{array}{r}1 \\
9.3\end{array}$ & -0.0 & 3.1 \\
\hline 4 & 13.6 & " & 8.9 & $\begin{array}{l}13.8 \\
13.1\end{array}$ & 46 & $\begin{array}{l}9.1 \\
5.4\end{array}$ & $\stackrel{6}{\Xi}$ & & & 8.0 & -4.5 & 4.5 & $\begin{array}{l}.0 \\
8.7\end{array}$ & a & $\begin{array}{l}2.7 \\
3.4\end{array}$ & $\begin{array}{l}8.0 \\
8.0\end{array}$ & 66 & 1.s \\
\hline 5 & 12.6 & " & 7.9 & 12.7 & " & 8.0 & 鉨 & & & 9.8 & " & 5.3 & 9.0 & -5.4 & 3.6 & $\therefore .9$ & $" 6$ & 2.7 \\
\hline 6 & 10.1 & " & 5.4 & 12.1 & -4.8 & 7.3 & $\approx$ & & & 10.3 & "6 & 5.5 & 111.2 & $" 6$ & 4.5 & 11.2 & -6.1 & ๖.1 \\
\hline 7 & 9.5 & 6 & 4.5 & 11.8 & " & 7.0 & & & & 11.0 & -4.6 & 6.4 & 11.6 & 46 & 6.2 & 13.0 & " & 6.9 \\
\hline 8 & $\begin{array}{l}9.0 \\
9.0\end{array}$ & 66 & $\begin{array}{l}4.3 \\
4.3\end{array}$ & $\begin{array}{l}11.6 \\
11.6\end{array}$ & -4.9 & 6.7 & & & & 11.3 & "6 & 6.7 & 12.3 & 6 & 6.9 & 14.2 & " & 8.1 \\
\hline 9 & 9.11 & " & 4.3 & 11.4 & 4 & 6.5 & & & & 12.0 & " & 7.4 & 12.9 & 6 & 7.5 & 15.5 & " & 9.4 \\
\hline & 0.1 & 66 & 4.4 & 11.6 & " & 6.7 & & & & 12.5 & "6 & 7.9 & 13.3 & $" 6$ & 7.9 & & & \\
\hline 10 & 9.5 & " & 4.5 & 12.0 & -5.0 & 7.0 & & & & $\begin{array}{l}12.5 \\
12.5\end{array}$ & -4.7 & $\begin{array}{l}7.8 \\
7.8\end{array}$ & $\begin{array}{l}13.5 \\
13.5\end{array}$ & 6 & $\begin{array}{l}8.1 \\
8.1\end{array}$ & 14.6 & " & 8.5 \\
\hline 11 & 10.1 & "4 & 5.4 & 12.7 & " & 7.7 & & & & 12.0 & 16 & 7.3 & 13.3 & 6 & 7.9 & 14.0 & " & 7.9 \\
\hline Midn't & 11.1 & " & 6.4 & 13.3 & -5.1 & 8.2 & & & & 10.5 & -4.5 & 5.7 & 12.7 & -5.5 & 7.2 & 13.5 & -6.0 & 7.5 \\
\hline
\end{tabular}

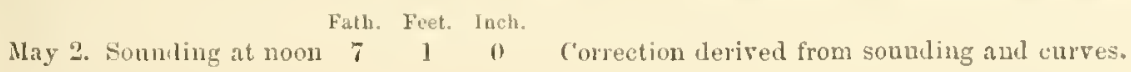

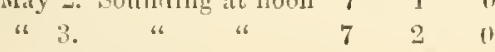

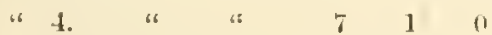

$$
\begin{aligned}
& \text { "5. " " " } \\
& \text { "i. No soundiug. } \\
& \text { "7. Sounding at noon ti } 3 \quad 0 \\
& \text { "8. " " }
\end{aligned}
$$

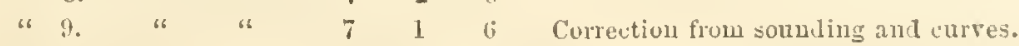


Series III.-Tidal Observations fron A pril 20 to August 3, 1854.

IIourly observations on the pulley-gange. Adopted reading of mean level 7.0, expressed in units of the scale. Increasing numbers indicate rise of water.

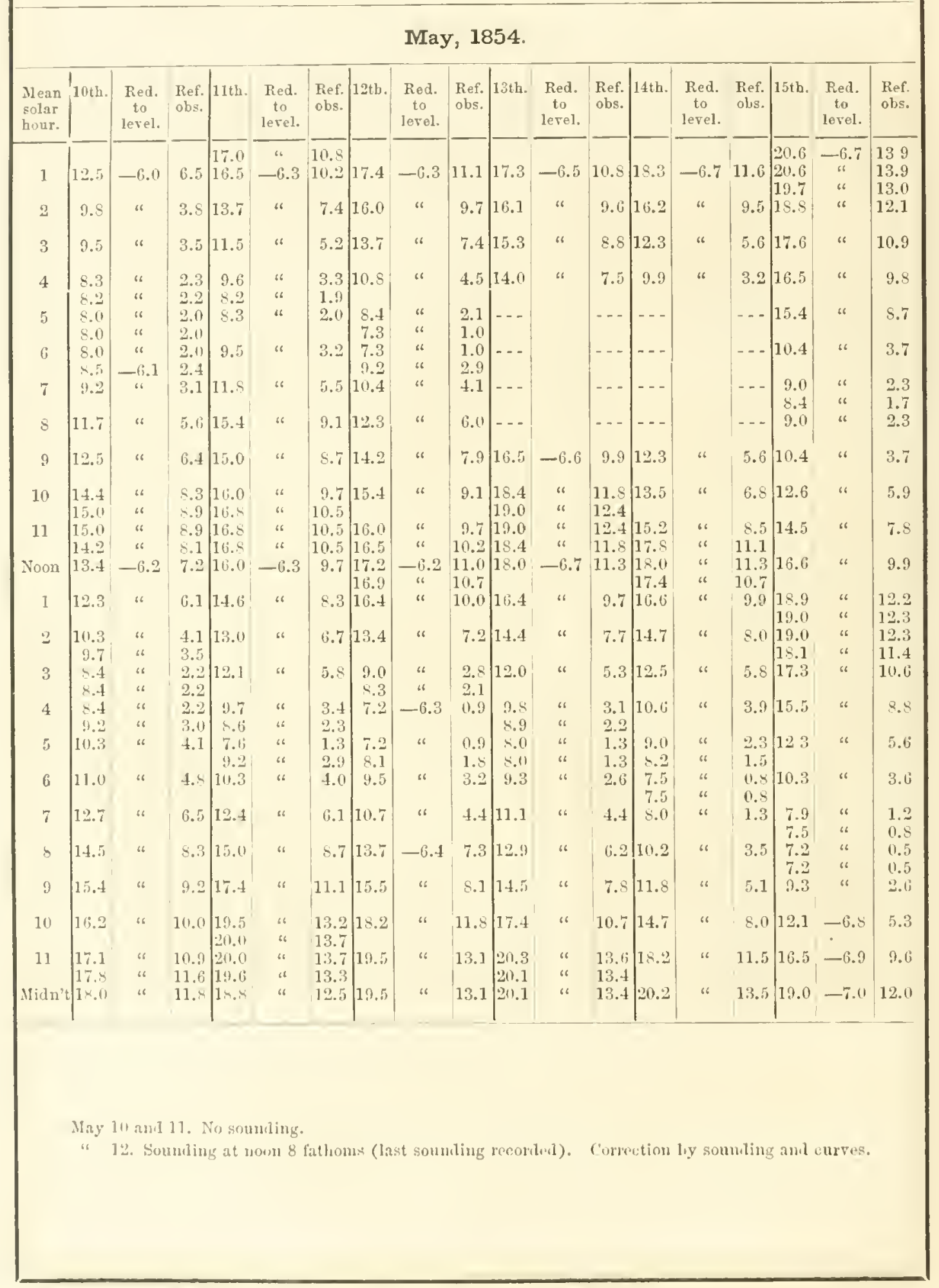


Series III.-Tidal Orservations froy A phil 20 to 1 ugugt $3,1854$.

IIourly observations on the pulley-gange. Adopted reading of mean level 7.0, expressed in units of the scale. Increasing numbers indieate rise of water.

May, 1854.

\begin{tabular}{|c|c|c|c|c|c|c|c|c|c|c|c|c|c|c|c|c|c|c|}
\hline $\begin{array}{l}\text { Mean } \\
\text { solar } \\
\text { hour. }\end{array}$ & $16 \mathrm{th}$. & $\begin{array}{l}\text { Ref. } \\
\text { obs. }\end{array}$ & 17 th. & $\begin{array}{l}\text { Ref. } \\
\text { obs. }\end{array}$ & $18 \mathrm{th}$. & $\begin{array}{l}\text { Ref. } \\
\text { obs. }\end{array}$ & $13 \mathrm{th}$. & $\begin{array}{l}\text { Ref. } \\
\text { obs. }\end{array}$ & 20 th. & $\begin{array}{l}\text { Ref. } \\
\text { obs. }\end{array}$ & 2 Ist. & $\begin{array}{l}\text { Pef. } \\
\text { obs. }\end{array}$ & $22 d$. & $\begin{array}{l}\text { Ref. } \\
\text { abs. }\end{array}$ & $23 r l$. & $\begin{array}{l}\text { Ref. } \\
\text { obs. }\end{array}$ & $24 \mathrm{th}$. & $\begin{array}{l}\text { Ref. } \\
\text { obs. }\end{array}$ \\
\hline \multirow[b]{2}{*}{1} & 19.6 & 12.4 & & & & & & & 12.0 & 5.2 & & & 10.3 & 3.4 & & & & \\
\hline & $\begin{array}{l}19.6 \\
19.0\end{array}$ & $\begin{array}{l}12.4 \\
11.8\end{array}$ & 18.0 & 10.8 & 17.6 & 10.4 & 14.0 & 6.9 & 12.3 & 5.5 & $\begin{array}{l}12.0 \\
11.6\end{array}$ & $\begin{array}{l}5.2 \\
4.8\end{array}$ & $\begin{array}{l}10.3 \\
10.3\end{array}$ & $\begin{array}{l}3.4 \\
3.4\end{array}$ & 13.0 & 5.6 & 16.2 & 7.5 \\
\hline \multirow[t]{2}{*}{2} & 18.5 & 11.3 & 19.4 & 11.2 & 19.0 & 11.8 & 15.0 & 7.9 & 13.2 & 6.4 & 11.2 & 4.4 & 10.3 & 3.4 & 12.2 & 4.8 & 15.1 & 6.3 \\
\hline & & & 20.0 & 12.5 & 19.7 & 12.5 & & & & & 11.2 & 4.4 & 10.3 & 3.4 & 11.3 & 3.8 & & \\
\hline \multirow[t]{2}{*}{3} & 17.0 & 9.8 & 20.0 & 12.8 & 19.7 & 12.5 & 16.4 & 9.3 & 14.1 & 7.3 & 12.5 & 5.7 & 10.8 & 3.9 & 11.0 & 3.5 & 14.2 & 5.4 \\
\hline & & & 18.9 & 11.7 & 19.7 & 12.5 & & & & & & & & & 11.5 & 4.0 & 13.5 & 4.9 \\
\hline \multirow[t]{2}{*}{4} & 16.0 & 8.8 & 18.0 & 111.8 & 19.7 & 12.5 & 17.7 & 10.6 & 15.2 & 8.5 & 14.2 & 7.4 & 11.5 & 4.6 & 12.0 & 4.4 & 13.0 & 4.1 \\
\hline & & & & & 19.7 & 12.5 & 18.8 & 11.7 & & & & & & & & & 13.0 & 4.0 \\
\hline 5 & 14.4 & 7.2 & 16.2 & 9.0 & 19.7 & 12.5 & 18.8 & 11.7 & 15.5 & 8.8 & 15.6 & 8.8 & 13.8 & 5.9 & 13.7 & 6.1 & 13.0 & 4.11 \\
\hline \multirow[t]{2}{*}{6} & 12.5 & 5.3 & 14.3 & 7.1 & 17.8 & 10.6 & 19.8 & 11.7 & 15.5 & 8.8 & 16.8 & 10.0 & 15.2 & 8.2 & 15.2 & 7.5 & 14.2 & $\begin{array}{l}4.0 \\
5.1\end{array}$ \\
\hline & & & & & & & 18.0 & 11.0 & 16.5 & 9.8 & 17.4 & 10.6 & 16.5 & 3.5 & & & & \\
\hline \multirow[t]{2}{*}{7} & 10.0 & 2.9 & 12.6 & 5.4 & 15.8 & S.6 & 17.4 & 10.4 & 16.5 & 9.8 & 17.9 & 11.1 & 17.6 & 10.6 & 17.2 & 9.5 & 16.6 & 7.5 \\
\hline & 9.5 & 2.3 & & & & & & & 16.5 & 9.8 & 17.9 & 11.1 & 17.6 & 10.6 & 15.2 & 10.4 & 17.6 & 8.6 \\
\hline \multirow[t]{2}{*}{8} & 9.0 & 1.8 & 11.3 & 4.1 & 14.3 & 7.1 & 16.0 & 9.0 & 16.5 & 9.5 & 17.9 & 11.1 & 17.6 & 10.6 & 19.1 & 11.3 & 18.6 & 9.4 \\
\hline & $\begin{array}{r}9.7 \\
90\end{array}$ & 2.5 & 10.2 & 3.0 & & & & & 15.8 & 9.1 & 17.6 & 10.8 & 17.6 & 10.6 & 19.1 & 11.2 & $1 \% .1$ & 9.9 \\
\hline 9 & 10.0 & 2.8 & $\begin{array}{r}9.0 \\
10.2\end{array}$ & $\begin{array}{l}1.8 \\
3.0\end{array}$ & 13.0 & 5.8 & 14.4 & 7.4 & 14.9 & 8.2 & 17.0 & 10.2 & 17.0 & 10.6 & 18.7 & 10.5 & 17.5 & 8.5 \\
\hline 10 & 10.8 & 3.7 & 11.1 & 3.9 & 10.0 & 2.5 & 12.4 & 5.4 & 13.6 & 6.9 & 15.7 & 8.9 & 16.5 & 9.5 & 17.5 & 9.5 & 16.7 & 7.4 \\
\hline \multirow[t]{2}{*}{11} & 12.4 & 5.2 & 13.4 & 6.2 & 9.0 & 1.8 & 11.7 & 4.7 & 12.8 & 6.1 & 14.6 & 7.8 & 15.1 & 8.1 & 16.0 & 8.0 & 15.1 & 5.8 \\
\hline & 110 & 77 & 11 & & 10.3 & 3.1 & 10.6 & 3.6 & 1.9) & & 120 & 60 & 137 & fi- & 15.3 & 73 & 115 & 50 \\
\hline Noon & 14.3 & 7.1 & 14.6 & 7.4 & 11.6 & 4.4 & 10.5 & $\begin{array}{l}3.01 \\
3.5\end{array}$ & 12.1 & 5.4 & 12.5 & 0.0 & 15.6 & 0.8 & 15.0 & 1.0 & 14.0 & 0.2 \\
\hline 1 & 16.2 & 9.0 & 15.9 & 8.7 & 14.0 & 6.8 & 10.8 & 3.8 & 11.5 & 4.8 & 11.2 & $4: 4$ & 12.0 & 5.0 & 14.2 & 6.2 & 13.5 & 4.5 \\
\hline \multirow{2}{*}{2} & $\begin{array}{l}17.0 \\
17.6\end{array}$ & $\begin{array}{r}9.8 \\
10.4\end{array}$ & 17.6 & 10.4 & 15.2 & 8.0 & 11.8 & 4.8 & 11.0 & 4.3 & $\begin{array}{l}10.5 \\
10.5\end{array}$ & $\begin{array}{l}4.0 \\
3.7\end{array}$ & 11.0 & 4.0 & 13.0 & 5.0 & 13.1 & 3. \\
\hline & 17.6 & 10.4 & 17.8 & 10.6 & & & & & 10.5 & 3.8 & 10.5 & 3.7 & 10.4 & 3.4 & & & & \\
\hline \multirow[t]{2}{*}{3} & 17.6 & 10.4 & 17.8 & 10.6 & 16.3 & 9.1 & 12.8 & 5.8 & 10.5 & 3.8 & 10.5 & 3.7 & 9.5 & 2.5 & 12.0 & 3.9 & 12.1 & 2.8 \\
\hline & & & 16.7 & 9.5 & 16.8 & 9.6 & & & 10.5 & 3.8 & 11.0 & 4.2 & 9.5 & 2.5 & 11.5 & 3.4 & 12.1 & 2.8 \\
\hline \multirow[t]{2}{*}{4} & 16.3 & 9.1 & 16.0 & 8.8 & 16.4 & 9.6 & 14.2 & 7.2 & 10.8 & 4.1 & 11.7 & 4.9 & 11.0 & 4.0 & 11.5 & 3.3 & 11.3 & 2.0 \\
\hline & 144 & $7 ?$ & $1+0$ & 68 & 16.8 & 8.6 & 107 & 87 & 19) & 60 & 126 & 58 & 717 & 47 & 11.5 & 3.3 & $11 . .3$ & 2.0 \\
\hline 5 & 14.4 & 1.2 & 14.0 & 0.0 & 101.0 & 0.4 & 16.5 & $\begin{array}{l}8.1 \\
9.5\end{array}$ & 12.1 & 0.0 & 12.0 & 0.0 & 11.6 & 4.1 & 12.4 & 4.0 & 12.00 & 3.2 \\
\hline 6 & 12.0 & 4.8 & 12.8 & 5.6 & 14.0 & 6.8 & 17.2 & 10.2 & 14.3 & 7.6 & 13.7 & 6.9 & 13.1 & 6.1 & 14.11 & 5.6 & 14.2 & 4.9 \\
\hline 7 & $\begin{array}{r}10.8 \\
9.5\end{array}$ & $\begin{array}{l}3.6 \\
2.3\end{array}$ & 10.6 & 3.4 & 13.3 & 6.1 & 16.5 & 9.5 & 14.9 & 8.2 & 14.5 & 7.7 & 14.1 & 7.0 & 15.5 & 7.0 & 16.5 & 7.3 \\
\hline & 9.5 & 2.3 & 10.0 & 2.8 & & & & & 15.2 & 8.5 & 15.0 & 8.2 & & & & & & \\
\hline 8 & 9.8 & 2.6 & 9.2 & 2.0 & 12.4 & 5.2 & 15.7 & 8.7 & 15.4 & 8.7 & 15.4 & 5.6 & 16.4 & 9.3 & 16.6 & 8.1 & 18.4 & 9.2 \\
\hline & & & 9.2 & 2.0 & 12.0 & 4.9 & & & 15.4 & 8.7 & 15.4 & 8.6 & 16.5 & 2.3 & & & & \\
\hline 9 & 11.7 & 4.5 & 10.4 & 3.2 & 11.3 & 4.2 & 15.0 & 8.1 & 15.4 & 8.7 & 15.4 & 8.6 & 16.5 & 9.3 & 18.3 & 9.7 & 19.4 & 10.2 \\
\hline 10 & 13.5 & 6. 3 & 120 & 48 & 11.3 & $\begin{array}{r}4.2 \\
+4\end{array}$ & 140 & 7.1 & 14.6 & $\begin{array}{l}7.9 \\
7.4\end{array}$ & 15.2 & $\begin{array}{l}8.4 \\
7.9\end{array}$ & $\begin{array}{l}16.5 \\
16.5\end{array}$ & $\begin{array}{l}9.3 \\
9.3\end{array}$ & $\begin{array}{l}19.4 \\
200\end{array}$ & 10.8 & 20.1 & 109 \\
\hline & & & & & 11.8 & 4.7 & & 1.1 & & & & & 16.5 & 0.2 & 20.0 & 11.4 & 10.1 & 100 \\
\hline 11 & 15.3 & 8.1 & 13.6 & 6.4 & 12.4 & 5.3 & 13.0 & 6.1 & 13.3 & 6.5 & 14.0 & 7.2 & 16.3 & 9.0 & 19.2 & 10.6 & 21.4 & 12.2 \\
\hline Midn't & 16.7 & 9.5 & 14.8 & 7.6 & 12. 3 & 5.7 & 12.5 & 5.7 & 12.4 & 5.6 & 12.6 & 5.8 & 15.0 & 7.7 & 18.0 & 9.3 & 22.1 & 12.9 \\
\hline Fro & abo & $\begin{array}{l}\text { out th } \\
\text { en be }\end{array}$ & $\begin{array}{l}\text { micle } \\
\text { w :- }\end{array}$ & Whe & & & 13) & & ri & & & & se & & litt & frou & & I. \\
\hline & & May & 16. Co & orrecti & $1011-$ & 7.2 & & & & & & May & $21 .\left({ }^{\circ}\right.$ & oryeti & ion - & (i.) & & \\
\hline & & . & 17. & " & & 7.2 & & & & & & “ & 22. & & & 7.11 & & \\
\hline & & "6 & 18. & " & & $-\pi .2$ & & & & & & " & 23. & & & 8.11 & & \\
\hline & & " & 19. & “" & & 7.11 & & & & & & " & 24. & " & & & & \\
\hline & & “ & & " & - & 6.7 & & & & & & & & & & & & \\
\hline & & & & & & & & & & & & & & - & & & & \\
\hline
\end{tabular}


Series III.-Tidal Observatrons from A pril 20 to A ugust 3, 1854.

Hourly obserrations on the pulley-gauge. Adopted reading of mean level 7.0, expressed in units of the seale. Inereasing numbers indicate rise of water.

\begin{tabular}{|c|c|c|c|c|c|c|c|c|c|c|c|c|c|c|c|c|c|c|}
\hline \multicolumn{15}{|c|}{ May, 1854.} & \multicolumn{4}{|c|}{ June, 1854.} \\
\hline $\begin{array}{l}\text { Mean } \\
\text { solar } \\
\text { bour. }\end{array}$ & 25 th. & $\begin{array}{l}\text { Ref. } \\
\text { obss. }\end{array}$ & $26 \mathrm{tb}$ & $\begin{array}{l}\text { Ref. } \\
\text { obs. }\end{array}$ & $2 \pi$ th. & $\begin{array}{l}\text { Ref. } \\
\text { obs. }\end{array}$ & 28 th. & $\begin{array}{l}\text { Ref. } \\
\text { obs. }\end{array}$ & $29 \mathrm{tb}$ & $\begin{array}{l}\text { Ref. } \\
\text { obs. }\end{array}$ & $30 \mathrm{th}$ & $\begin{array}{l}\text { Ref. } \\
\text { obs. }\end{array}$ & 31st. & $\begin{array}{l}\text { Ref. } \\
\text { obs. }\end{array}$ & 1st. & $\begin{array}{l}\text { Ref. } \\
\text { obs. }\end{array}$ & $2 \mathrm{~d}$. & $\begin{array}{l}\text { Ref. } \\
\text { obs. }\end{array}$ \\
\hline \multirow[t]{2}{*}{1} & $\begin{array}{l}19.5 \\
19.0\end{array}$ & $\begin{array}{r}10.6 \\
9.5\end{array}$ & $\begin{array}{l}17.4 \\
16.6\end{array}$ & $\begin{array}{l}8.5 \\
7.7\end{array}$ & $\mid \begin{array}{l}21.2 \\
21.2\end{array}$ & $\begin{array}{l}12.5 \\
12.5\end{array}$ & $\begin{array}{l}19.0 \\
19.0\end{array}$ & $\begin{array}{l}10.7 \\
10.7\end{array}$ & 18.4 & 10.8 & \begin{tabular}{|l|}
19.0 \\
19.5
\end{tabular} & $\begin{array}{l}11.5 \\
12.0\end{array}$ & 16.4 & 8.9 & 18.2 & 10.7 & 15.4 & 8.0 \\
\hline & & & & & 19.6 & 10.9 & 18.6 & 10.4 & & & 18.6 & 11.1 & & & 18.4 & 10.9 & & 0.0 \\
\hline 2 & 17.11 & 7.9 & 14.3 & 5.4 & 18.7 & 10.1 & 17.3 & 9.1 & 16.4 & 8.8 & 18.1 & 10.6 & 17.9 & 10.4 & 18.5 & 11.0 & 16.1 & 8.7 \\
\hline \multirow[t]{2}{*}{3} & 14.5 & 5.4 & 12.4 & 3.6 & 16.0 & 7.4 & 15.5 & 7.4 & 14.3 & 6.7 & 17.2 & 9.5 & 19.0 & 11.5 & 18.2 & 10.7 & 17.0 & 9.6 \\
\hline & & & 11.8 & 3.11 & & & & & & & & & 19.0 & 11.5 & & & 17.2 & 9.8 \\
\hline 4 & 12.11 & $\begin{array}{l}2.9 \\
2.0\end{array}$ & $\begin{array}{l}11.5 \\
11.5\end{array}$ & $\begin{array}{l}2.7 \\
2.7\end{array}$ & 14.7 & 6.1 & 12.4 & 4.3 & 12.1 & 4.5 & 16.1 & 8.6 & 18.2 & 10.7 & 17.8 & 10.3 & \begin{tabular}{|l}
17.2 \\
17.2
\end{tabular} & $\begin{array}{l}9.8 \\
9.8\end{array}$ \\
\hline \multirow[t]{2}{*}{5} & $\begin{array}{l}1.0 \\
11.0\end{array}$ & 2.0 & 11.5 & 2.7 & 12.5 & 3.9 & 10.8 & 2.8 & 10.0 & 2.5 & 14.3 & 0.8 & 17.2 & 9.7 & 16.2 & 8.7 & 17.0 & 9.7 \\
\hline & 11.0 & 2.0 & 11.5 & 2.7 & 11.2 & 2.6 & 10.0 & 2.0 & 9.0 & 1.5 & & & & & & & & \\
\hline i & 11.5 & 2.5 & 11.5 & 2.7 & 11.0 & 2.4 & 9.5 & 1.6 & 9.0 & 1.5 & 12.4 & 4.9 & 14.9 & 7.4 & 14.3 & 6.8 & 15.6 & 8.3 \\
\hline \multirow[t]{2}{*}{7} & 13.5 & 4.5 & 12.5 & 3.7 & 12.4 & $\begin{array}{l}2.4 \\
3.8\end{array}$ & 9.5 & $\begin{array}{l}1.0 \\
1.6\end{array}$ & 9.0 & 1.5 & 10.2 & 2.7 & 12.9 & 5.4 & 13.4 & 5.9 & 14.2 & 6.9 \\
\hline & & & & & & & 9.5 & 1.6 & 9.0 & 1.5 & 10.0 & 2.5 & & & & & & \\
\hline 8 & 15.1 & 0.1 & 14.3 & 5.5 & 14.1 & 5.5 & 10.1 & 2.3 & 9.0 & 1.5 & 10.0 & 2.5 & 12.0 & 4.5 & 12.6 & 5.1 & 12.8 & 5.5 \\
\hline \multirow[t]{2}{*}{9} & 17.4 & 8.4 & 15.2 & 6.4 & 15.2 & 6.7 & 11.7 & 3.9 & 10.2 & $\begin{array}{l}1.0 \\
2.7\end{array}$ & 10.0 & $\begin{array}{l}2.5 \\
2.5\end{array}$ & $\begin{array}{l}11.2 \\
10.0\end{array}$ & $\begin{array}{l}3.7 \\
2.5\end{array}$ & $\begin{array}{l}12.0 \\
11.5\end{array}$ & $\begin{array}{l}4.0 \\
4.0\end{array}$ & 12.0 & 4.7 \\
\hline & 18.2 & 0.7 & & & & & & & & & 11.3 & 3.8 & 10.0 & 2.5 & 11.5 & 4.0 & 11.3 & 4.0 \\
\hline \multirow{2}{*}{10} & 19.0 & 10.0 & 16.3 & 7.5 & 17.3 & 8.8 & 13.0 & 5.2 & 12.4 & 4.9 & 12.4 & 4.9 & 10.5 & 3.0 & 11.5 & 4.0 & 11.0 & 3.7 \\
\hline & & 10.0 & & & 18.2 & 9.7 & $11+7$ & & & & & & & & 11.5 & 4.0 & 11.0 & 3.7 \\
\hline 11 & 18.4 & 8.4 & 17.2 & 8.4 & $\begin{array}{l}18.2 \\
18.0\end{array}$ & $\begin{array}{l}9.7 \\
9.5\end{array}$ & $\begin{array}{l}1+.7 \\
15.4\end{array}$ & $\begin{array}{l}6.9 \\
7.6\end{array}$ & 14.4 & $6 . ?$ & 13.8 & 6.3 & 12.6 & 5.1 & 12.0 & 4.5 & $\begin{array}{l}11.0 \\
11.5\end{array}$ & 3.7 \\
\hline \multirow[t]{2}{*}{ Noon } & 16.0 & 7.0 & 18.4 & 9.6 & 17.5 & 9.0 & 16.5 & 8.7 & 15.5 & 8.0 & 14.9 & 7.4 & 13.7 & 6.2 & 13.0 & 5.5 & 11.8 & 4.5 \\
\hline & & & 18.5 & $\because 7$ & & & 15.7 & 7.9 & $1 t 5.0$ & 8.5 & & & & & & & & \\
\hline 1 & 15.0 & 6.0 & 18.5 & 9.7 & 16.6 & 8.1 & 15.1. & 7.3 & 16.0 & 8.5 & 15.3 & 7.5 & 14.8 & 7.3 & 14.5 & 7.0 & 12.4 & 5.1 \\
\hline \multirow[t]{2}{*}{2} & 14.0 & 5.0 & $\begin{array}{l}17.1 \\
17.1\end{array}$ & $\begin{array}{l}8.9 \\
8.3\end{array}$ & 14.2 & 5.7 & 13.5 & 5.7 & $\begin{array}{l}1.6 .6 \\
15.1\end{array}$ & 8.1 & $\left|\begin{array}{l}16.0 \\
16.0\end{array}\right|$ & $\begin{array}{l}8.5 \\
8.5\end{array}$ & $\begin{array}{l}15.4 \\
16.0\end{array}$ & 8.5 & 14.6 & 7.1 & 12.8 & 5.5 \\
\hline & & & & & & & & & & & 16.0 & 8.5 & 16.0 & 8.5 & & & & \\
\hline 3 & 13.2 & 4.2 & 16.0 & 7.2 & 12.3 & 3.8 & 11.7 & 3.9 & 14.2 & 6.7 & 16.0 & 8.5 & 16.0 & 8.5 & 15.0 & 7.5 & 13.5 & 6.2 \\
\hline 4 & 12.0 & 3.0 & 14.8 & 6.0 & $\begin{array}{l}\because 00 \\
9.0\end{array}$ & $\begin{array}{l}1.1 \\
0.5\end{array}$ & $\begin{array}{l}10.0 \\
10.0\end{array}$ & $\begin{array}{l}3.1 \\
2.3\end{array}$ & 12.3 & 4.8 & \begin{tabular}{|l|}
10.2 \\
14.2
\end{tabular} & $\begin{array}{l}7.7 \\
6.7\end{array}$ & $\begin{array}{l}15.4 \\
15.0\end{array}$ & 7.5 & $\begin{array}{l}10.6 \\
15.5\end{array}$ & $\begin{array}{l}8.0 \\
8.0\end{array}$ & 14.4 & 7.2 \\
\hline & 11.4 & 2.4 & & & 8.1 & 0.5 & 10.0 & 2.3 & & & & & & & 15.5 & 8.0 & 15 & 7.8 \\
\hline 5 & 11.0 & 2.11 & 13.6 & 4.5 & 9.5 & 1.0 & 10.0 & 2.3 & 11.6 & 4.1 & 12.8 & 5.3 & 13.5 & 6.0 & 15.5 & 8.0 & 15.0 & 7.8 \\
\hline & 11.0 & 2.0 & 13.0 & 4.2 & & & 10.0 & 2.3 & & & 11.2 & 3.7 & & & 15.2 & 7.7 & 14.3 & 7.1 \\
\hline 6 & $11.1)$ & 2.1 & 12.2 & 3.4 & 10.0 & 1.5 & 11.3 & 3.6 & 10.2 & 2.7 & 10.0 & 2.5 & 12.7 & 5.2 & 15.0 & 7.6 & 14.0 & 6.8 \\
\hline & 12.1 & 3.1 & 12.2 & 3.4 & & & & & 9.5 & 2.0 & 10.0 & 2.5 & & & & & & \\
\hline 7 & 13.3 & 4.3 & 12.2 & 3.4 & 10.4 & 1.8 & 12.3 & 4.6 & 9.1 & 1.6 & 10.0 & 2.5 & 11.7 & 4.2 & 14.2 & 6.8 & 13.4 & 6.2 \\
\hline$S$ & 15.7 & 6.5 & $\begin{array}{l}12.6 \\
13.2\end{array}$ & $\begin{array}{l}3.8 \\
4.5\end{array}$ & 12.1 & 3.6 & 14.2 & 6.5 & $\begin{array}{l}9.1 \\
9.1\end{array}$ & $\begin{array}{l}1.6 \\
1.6\end{array}$ & $\mid \begin{array}{l}10.0 \\
11.0\end{array}$ & $\begin{array}{l}2.5 \\
3.5\end{array}$ & $\begin{array}{l}11.5 \\
11.0\end{array}$ & $\begin{array}{l}4.0 \\
3.5\end{array}$ & 13.0 & 5.6 & 12.8 & 5.6 \\
\hline & & & & & & & & & 10.2 & 2.7 & & & 11.0 & 3.5 & & & & \\
\hline 3 & 17.4 & 8.5 & 15.3 & 6.6 & 14.2 & 5.8 & 15.0 & 7.3 & 11.3 & 3.8 & 13.0 & 5.5 & 11.0 & 3.5 & 12.0 & 4.6 & 12.4 & 5.2 \\
\hline 10 & 19.5 & 10.6 & 17.6 & 8.9 & 16.4 & 8.0 & 16.1 & 8.4 & 13.7 & 6.2 & 14.4 & 6.9 & 12.0 & 4.5 & 11.0 & 3.6 & 12.0 & 4.8 \\
\hline & 19.7 & 10.8 & & & & & & & & & & & & & 11.5 & 4.1 & 12.0 & 4.8 \\
\hline 11 & 19.7 & 10.8 & $\begin{array}{l}19.4 \\
201,1\end{array}$ & 10.7 & \begin{tabular}{|l|}
18.1 \\
18.7
\end{tabular} & $\begin{array}{r}9.8 \\
10.4\end{array}$ & $\begin{array}{l}17.4 \\
18.0\end{array}$ & $\begin{array}{r}9.7 \\
10.3\end{array}$ & 16.2 & 8.7 & 15.1 & 7.6 & 14.2 & 0.7 & 12.2 & 4.8 & $\begin{array}{l}12.0 \\
12.3\end{array}$ & $\begin{array}{l}4.8 \\
5.1\end{array}$ \\
\hline Mirln't & 19.7 & 10.8 & 201.1 & 11.4 & 19.11 & 10.7 & 18.6 & 10.9 & 17.4 & 9.9 & 16.2 & 8.7 & 15.8 & 8.3 & 14.3 & 6.9 & 12.7 & 5.5 \\
\hline & & May & $25.1 \mathrm{n}$ & orrectis & In & 1.11 & & & & & & Bl:y : & 80.10 & orTweti & $i \omega n-7$ & & & \\
\hline & & " & 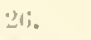 & " & -8 & & & & & & & " : & 31. & " & & & & \\
\hline & & " & 27. & “ & -8 & 4.5 & & & & & & Inue & 1. & $"$ & -7 & 7.5 & & \\
\hline & & “ & $2 k$. & " & -7 & & & & & & & " & 2. & " & -7 & 7.3 & & \\
\hline & & " & 。 & " & -7 & 7.5 & & & & & & & & & & & & \\
\hline
\end{tabular}


Series III.-Thdal, Observations frovi April 20 to August 3, 1854.

Hourly observations on the pulley-gange. Adopted reading of mean level 7.0, cxpressed in units of the seale. Inereasing numbers indieate rise of water.

\section{June, 1854.}

\begin{tabular}{|c|c|c|c|c|c|c|c|c|c|c|c|c|c|c|c|c|c|c|}
\hline $\begin{array}{l}\text { Mean } \\
\text { solar } \\
\text { hour. }\end{array}$ & $3 d$. & $\begin{array}{l}\text { Ref. } \\
\text { obs. }\end{array}$ & 4th. & $\begin{array}{l}\text { Ref. } \\
\text { obs. }\end{array}$ & 5 th. & $\begin{array}{l}\text { Ref. } \\
\text { obs. }\end{array}$ & 6th. & $\begin{array}{l}\text { Ref. } \\
\text { obss. }\end{array}$ & Tth. & $\begin{array}{l}\text { Ref. } \\
\text { obs. }\end{array}$ & 8th. & $\begin{array}{l}\text { Ref. } \\
\text { obs. }\end{array}$ & 9th. & $\begin{array}{l}\text { Ref. } \\
\text { obs. }\end{array}$ & 10 th. & $\begin{array}{l}\text { Ref. } \\
\text { obs. }\end{array}$ & 11th. & \\
\hline 1 & 14.2 & 7.1 & 13.0 & 6.3 & 12.4 & 6.0 & $\begin{array}{l}12.5 \\
12.5\end{array}$ & \begin{tabular}{|l|}
6.1 \\
6.1
\end{tabular} & 12.3 & 5.6 & 12.8 & 5.5 & - . & $\cdots$ & 16.4 & 8.2 & $\begin{array}{l}18.0 \\
19.0 \\
19.9\end{array}$ & \\
\hline 2 & 15.8 & 8.7 & 14.0 & 7.4 & 12.8 & 6.4 & $\begin{array}{l}12.5 \\
12.9\end{array}$ & 6.1 & 12.5 & 5.8 & $\begin{array}{l}11.8 \\
11.4\end{array}$ & $\begin{array}{l}4.4 \\
4.0\end{array}$ & $\cdots$ & -.. & 14.9 & 6.7 & 18.0 & \\
\hline 3 & 16.4 & 9.3 & 15.2 & 8.6 & 13.8 & 7.4 & 13.6 & 7.2 & 12.0 & 5.3 & 11.4 & 4.0 & - - & -.. & 12.1 & 3.9 & 15.2 & \\
\hline 4 & 16.6 & 9.6 & 16.0 & 9.4 & 15.2 & 8.8 & 14.2 & 7.8 & 11.0 & 4.3 & 14.5 & 7.0 & $\cdots$ & -.. & 12.0 & 3.7 & 13.2 & \\
\hline 5 & $\cdots$ & $\cdots$ & $\cdots$ & ... & 15.4 & 9.0 & 14.8 & 8.4 & 15.6 & 8.8 & 14.7 & 7.4 & - - & -- & 10.4 & 2.1 & 10.4 & \\
\hline 6 & $\ldots$ & $\ldots$ & $\ldots$ & ... & 15.8 & 9.4 & 15.0 & 8.6 & 16.0 & 9.2 & 15.0 & 7.7 & - - - & ... & 10.9 & 2.6 & 10.4 & \\
\hline 7 & $-\cdots$ & $\cdots$ & $\ldots$ & ... & 16.0 & 9.6 & 15.6 & 9.2 & 17.0 & ? & 15.6 & 8.2 & - - & $\cdots$ & 11.5 & 3.2 & 10.4 & \\
\hline 8 & - . & $\cdots$ & $\cdots$ & -.. & 16.1 & 9.7 & 16.0 & 9.6 & 15.5 & 8.7 & 16.8 & 9.3 & - - & - - & 11.1 & $?$ & 13.2 & \\
\hline S & 11.5 & 4.5 & ... & ... & 14.1 & 7.7 & $\begin{array}{l}16.5 \\
15.5\end{array}$ & $\begin{array}{r}10.1 \\
9.1\end{array}$ & $\begin{array}{l}17.1 \\
16.8\end{array}$ & $\begin{array}{r}10.2 \\
9.9\end{array}$ & 16.6 & 9.1 & - . & $\ldots$ & $\ldots$ & $\ldots$ & 16.0 & \\
\hline 10 & 12.0 & 5.0 & $\cdots$ & -. & 12.2 & 5.8 & 14.8 & 8.4 & 15.5 & 8.5 & 16.2 & 8.6 & 16.0 & 8.0 & $\begin{array}{l}20.0 \\
19.2\end{array}$ & $\begin{array}{l}11.7 \\
10.9\end{array}$ & 18.0 & \\
\hline 11 & 12.5 & 5.5 & -.- & --- & $\begin{array}{l}11.5 \\
11.3\end{array}$ & $\begin{array}{l}5.1 \\
4.9\end{array}$ & 14.5 & 8.1 & 14.5 & 7.5 & 13.4 & 5.8 & 13.9 & 5.9 & 18.0 & 9.7 & 18.0 & \\
\hline Noon & 13.0 & 6.0 & -.. & -.. & 11.1 & $\begin{array}{l}4.7 \\
4.7\end{array}$ & 13.3 & 6.9 & 12.9 & 5.9 & 12.5 & 4.9 & 12.6 & 4.6 & 17.6 & 9.3 & 17.2 & \\
\hline 1 & 13.5 & 6.5 & $\cdots$ & $\ldots$ & $\begin{array}{l}11.1 \\
11.2\end{array}$ & $\begin{array}{l}4.1 \\
4.7 \\
4.8\end{array}$ & $\begin{array}{l}11.6 \\
10.8\end{array}$ & $\begin{array}{l}5.2 \\
4.4\end{array}$ & 11.9 & 4.9 & $\begin{array}{l}11.8 \\
10.4\end{array}$ & $\begin{array}{l}4.2 \\
2.7\end{array}$ & & $\begin{array}{l}4.0 \\
2.3\end{array}$ & 16.4 & 8.1 & 16.8 & \\
\hline 2 & 14.0 & 7.0 & 11.4 & 5.0 & 11.2 & 4.8 & $\begin{array}{l}10.5 \\
10.5\end{array}$ & $\begin{array}{l}4.0 \\
4.0\end{array}$ & $\begin{array}{l}11.4 \\
10.5\end{array}$ & & 9.5 & 1.8 & 11.0 & 3.0 & 14.7 & 6.4 & 16.0 & \\
\hline 3 & & $\begin{array}{l}7.6 \\
8.1\end{array}$ & 12.4 & 6.0 & 11.4 & 5.0 & $\begin{array}{l}10.5 \\
10.9\end{array}$ & $\begin{array}{l}4.0 \\
4.4\end{array}$ & $\begin{array}{l}10.5 \\
11.5\end{array}$ & $\begin{array}{l}3.4 \\
4.4\end{array}$ & $\begin{array}{l}9.5 \\
9.8\end{array}$ & $\begin{array}{l}1.8 \\
2.1\end{array}$ & 9.4 & 1.4 & 13.2 & 4.9 & 15.0 & \\
\hline 4 & $\begin{array}{l}15.0 \\
15.0\end{array}$ & $\begin{array}{l}8.1 \\
8.1\end{array}$ & 13.6 & 7.2 & 12.4 & 6.0 & 11.4 & 4.3 & 12.6 & 5.5 & 10.5 & 2.8 & 10.3 & 2.3 & 11.7 & 3.3 & 14.2 & \\
\hline 5 & & 8.0 & 14.4 & 8.0 & 13.6 & 7.2 & 13.2 & 6.6 & 14.0 & 6.9 & 11.9 & 4.1 & 11.4 & 3.4 & $\begin{array}{r}10.2 \\
9.6\end{array}$ & $\begin{array}{l}1.8 \\
1.2\end{array}$ & 12.9 & \\
\hline 6 & 14.6 & 7.8 & 14.4 & 8.0 & 14.8 & 8.4 & 14.8 & 8.2 & 14.7 & 7.5 & 13.1 & 5.3 & 12.6 & 4.5 & 9.0 & 0.6 & 12. & \\
\hline 7 & 14.0 & 7.2 & 15.0 & 8.6 & 14.4 & 8.0 & 16.2 & 9.6 & 17.0 & 9.8 & 14.0 & 6.2 & 16.4 & 8.3 & 12.0 & 3.6 & 11.5 & \\
\hline 8 & 13.4 & 6.6 & 15.0 & 8.6 & 15.2 & 8.8 & 17.0 & 10.4 & 18.0 & 10.8 & 16.2 & 8.4 & 19.5 & 11.4 & 15.4 & 7.0 & 13.6 & \\
\hline 9 & & $\begin{array}{l}6.2 \\
5.2\end{array}$ & 14.0 & 7.6 & 15.6 & 9.2 & 16.6 & 10.0 & 18.4 & 11.1 & 18.6 & 10.8 & 21.0 & 12.9 & -.. & $\ldots$ & 16.2 & \\
\hline 10 & $\begin{array}{l}12.0 \\
12.0\end{array}$ & $\begin{array}{l}5.2 \\
5.2\end{array}$ & 13.0 & 6.6 & 14.6 & 8.2 & 16.2 & 9.6 & 18.0 & 10.7 & 19.4 & 11.6 & 20.6 & 12.5 & $\cdots$ & -.. & 19.2 & \\
\hline 11 & 12.3 & 5.6 & 12.5 & 6.1 & 13.2 & 6.8 & 16.2 & 9.6 & 17.2 & 9.9 & 19.0 & 11.2 & 20.0 & 11.9 & -- & $\cdots$ & 20.2 & \\
\hline Midn't & 13.0 & 6.3 & 12.0 & 5.6 & 12.6 & 6.2 & 15.2 & 8.6 & 14.0 & 6.7 & 18.0 & 10.2 & -.- & ... & ... & $\ldots$ & 21.4 & \\
\hline
\end{tabular}

Jane 3. Correction - 70

"6. " " 6.6 .4

"9. $6 \quad-8.0$

" $10.4 \quad-\$ .3$

"11. " 1 - 1.6
June 5. Correction -6.4

June 4. Correction -6.4

"7. " 7 . 7.0

The record on this day is defective.

Readings between the full hours are less frequent than before, and are generally [given only near high or low water. 
Series III.-Tidal Observatrons frosi A pril 20 to August 3, 1854.

Hourly ouservations on the pulley-gange. Adopted reading of mean level 7.0 , expressed in nnits of the scale. Increasing numbers indicate rise of water.

\section{June, 1854.}

\begin{tabular}{|c|c|c|c|c|c|c|c|c|c|c|c|c|c|c|c|c|c|c|}
\hline $\begin{array}{l}\text { Mean } \\
\text { solar } \\
\text { hour. }\end{array}$ & $12 \mathrm{th}$. & $\begin{array}{l}\text { Ref. } \\
\text { obs. }\end{array}$ & $13 \mathrm{th}$. & $\begin{array}{l}\text { Ref. } \\
\text { obs. }\end{array}$ & 14 th. & $\begin{array}{l}\text { Fef. } \\
\text { obs. }\end{array}$ & 15th. & $\begin{array}{l}\text { Ref. } \\
\text { obs. }\end{array}$ & 16th.' & $\begin{array}{l}\text { Ref. } \\
\text { abs. }\end{array}$ & 17th. & $\begin{array}{l}\text { Ref. } \\
\text { obs. }\end{array}$ & 18th. & $\begin{array}{l}\text { Ref. } \\
\text { obs. }\end{array}$ & 19 th. & $\begin{array}{l}\text { Ref. } \\
\text { obs. }\end{array}$ & 20th. & $\begin{array}{l}\text { Ref. } \\
\text { obs. }\end{array}$ \\
\hline 1 & $\begin{array}{l}23.0 \\
21.5\end{array}$ & $\begin{array}{l}13.2 \\
12.7\end{array}$ & $\begin{array}{l}21.0 \\
31.0\end{array}$ & $\begin{array}{l}12.1 \\
12.1\end{array}$ & $\begin{array}{l}21.0 \\
21.0\end{array}$ & $\begin{array}{l}12.1 \\
12.1\end{array}$ & $\begin{array}{l}19.2 \\
20.1\end{array}$ & $\begin{array}{l}10.4 \\
11.3\end{array}$ & 16.0 & 7.3 & 17.3 & 8.6 & 14.4 & 6.1 & 14.0 & 6.2 & 13.5 & $\begin{array}{l}5.8 \\
5.3\end{array}$ \\
\hline 2 & 19.8 & 11.0 & 19.9 & 11.0 & 21.0 & 12.1 & $\begin{array}{l}21.1 \\
21.1\end{array}$ & $\begin{array}{l}12.3 \\
12.3\end{array}$ & 17.2 & 8.5 & $\begin{array}{l}18.6^{\prime} \\
19.2\end{array}$ & $\begin{array}{r}8.9 \\
10.5\end{array}$ & 15.6 & 7.3 & 15.1 & 7.4 & $\begin{array}{l}12.9 \\
12.9 \\
12.9\end{array}$ & $\begin{array}{l}5.2 \\
5.2\end{array}$ \\
\hline 3 & 19.5 & 9.7 & $1 \S .2$ & 9.3 & 20.2 & 11.3 & $\begin{array}{l}21.1 \\
20.9\end{array}$ & 12.3 & $\begin{array}{l}19.0 \\
20.0\end{array}$ & $\begin{array}{l}10.3 \\
11.3\end{array}$ & 20.1 & 11.4 & 17.0 & 8.7 & 15.9 & 8.2 & 14.1 & 5.3 \\
\hline 4 & 15.2 & 6.4 & 18.0 & 9.1 & 19.6 & 10.7 & 20.2 & 11.5 & 20.4 & 11.7 & 19.9 & 11.3 & 18.2 & 9.9 & 16.2 & 8.6 & 11.0 & 6.2 \\
\hline 5 & 12.5 & 3.7 & 13.2 & 4.3 & 15.3 & 6.4 & 16.3 & 7.6 & 19.0 & 10.3 & 17.6 & 9.0 & $1 £ .4$ & 10.2 & 18.0 & 10.4 & 15. 6 & 7.8 \\
\hline 6 & 10.4 & 1.6 & 11.6 & 2.7 & 12.2 & 3.3 & 14.4 & 5.7 & 16.5 & 8.1 & 16.4 & 7.8 & 18.0 & 9.8 & 18.1 & 10.5 & 16.4 & 8.6 \\
\hline 7 & 10.4 & 1.6 & 10.0 & 1.1 & 10.5 & 1.6 & 11.5 & 2.8 & 14.3 & 5.6 & 14.2 & 5.6 & 17.5 & 2.3 & 18.0 & 10.4 & $17.6^{2}$ & 9.8 \\
\hline 8 & 13.2 & 4.4 & $\begin{array}{r}10.0 \\
9.1\end{array}$ & $\begin{array}{l}1.1 \\
0.2\end{array}$ & 10.5 & 1.6 & $\begin{array}{l}10.8 \\
10.4\end{array}$ & $\frac{2.1}{1.7}$ & 12.8 & 4.1 & $\begin{array}{l}12.4 \\
11.6\end{array}$ & $\begin{array}{l}3.5 \\
3.0\end{array}$ & 17.0 & 8.5 & 17.8 & 10.2 & 17.7 & 9.9 \\
\hline 9 & $1 \% 5$ & 6.7 & 10.0 & 1.1 & 12.6 & 3.7 & $\begin{array}{l}10.0 \\
11.0\end{array}$ & $\begin{array}{l}1.3 \\
2.3\end{array}$ & $\begin{array}{l}11.4 \\
11.2\end{array}$ & $\begin{array}{l}2.7 \\
2.5\end{array}$ & 11.4 & 2.5 & 13.4 & 5.3 & 15.6 & 8.0 & 17.6 & 9.5 \\
\hline 10 & 16.6 & 7.8 & 12.2 & 3.3 & 14.0 & 5.1 & 12.0 & 3.3 & 11.4 & 2.5 & 12.2 & 3.6 & 12.5 & 4.4 & 15.0 & 7.4 & 16.5 & 8.7 \\
\hline 11 & $\begin{array}{l}18.6 \\
19.2\end{array}$ & $\begin{array}{r}9.8 \\
10.4\end{array}$ & 16.4 & 7.5 & 16.0 & 7.1 & 14.5 & $5 . S$ & 13.0 & 4.3 & 12.6 & 4.0 & $\begin{array}{l}11.6 \\
11.2\end{array}$ & $\begin{array}{l}3.6 \\
3.2\end{array}$ & 14.0 & 6.4 & 14.5 & 7.0 \\
\hline Toon & 17.6 & B.S & $\begin{array}{l}18.4 \\
19.2\end{array}$ & $\begin{array}{r}9.5 \\
10.3\end{array}$ & $\begin{array}{l}17.6 \\
19.2\end{array}$ & $\begin{array}{r}8.7 \\
10.3\end{array}$ & $\begin{array}{l}16.2 \\
16.5\end{array}$ & $\begin{array}{l}7.5 \\
7.5\end{array}$ & 14.6 & 5.9 & 14.5 & 5.9 & 11.4 & 3.4 & 13.0 & 5.4 & 14.5 & 6.7 \\
\hline 1 & $15 . S$ & 7.0 & $\begin{array}{l}19.2 \\
19.2\end{array}$ & $\begin{array}{l}10.3 \\
10.3\end{array}$ & 19.1 & 10.2 & $\begin{array}{l}17.2 \\
17.2\end{array}$ & 8.5 & $\begin{array}{l}15.9 \\
16.8\end{array}$ & $\begin{array}{l}7.2 \\
8.1\end{array}$ & 15.9 & 7.3 & 12.0 & 4.0 & $\begin{array}{l}12.0 \\
11.6\end{array}$ & $\begin{array}{l}4.4 \\
4.0\end{array}$ & 14.0 & 6.2 \\
\hline 2 & 13.8 & 5.0 & 18.9 & 10.0 & 19.1 & 10.2 & $\begin{array}{l}17.2 \\
16.2\end{array}$ & $\begin{array}{l}8.5 \\
7.5\end{array}$ & $\begin{array}{l}17.0 \\
16.9\end{array}$ & $\begin{array}{l}8.9 \\
8.2\end{array}$ & $\begin{array}{l}17.3 \\
17.9\end{array}$ & $\begin{array}{l}8.7 \\
9.3\end{array}$ & 13.3 & 5.3 & 12.0 & 4.4 & 13.6 & 5.8 \\
\hline 3 & 13.0 & 4.2 & 17.6 & 8.7 & 17.4 & 8.5 & 15.5 & 6.8 & 16.2 & 7.5 & 17.5 & S.9 & 16.6 & 8.6 & 13.0 & 5.4 & $\begin{array}{l}13.0 \\
12.2\end{array}$ & $\begin{array}{l}5.2 \\
4.4\end{array}$ \\
\hline 4 & 11.6 & 2.8 & 16.2 & 7.3 & 16.0 & 7.1 & 15.0 & 6.3 & 15.5 & 6.8 & 17.0 & 8.1 & $\begin{array}{l}16.3 \\
16.0\end{array}$ & $\begin{array}{l}8.3 \\
8.0\end{array}$ & 14.0 & 6.4 & 12.4 & $4.6^{\circ}$ \\
\hline 5 & $\begin{array}{r}10.3 \\
9.6\end{array}$ & $\begin{array}{l}1.5 \\
0.5\end{array}$ & 14.8 & ว.9 & 14.2 & 5.3 & 13.4 & 4.7 & 14.5 & 6.1 & 16.6 & 8.0 & $\begin{array}{l}13.5 \\
15.0\end{array}$ & $\begin{array}{r}10.5 \\
7.0\end{array}$ & 15.0 & 7.4 & 14.0 & 6.1 \\
\hline 6 & $\begin{array}{l}9.2 \\
9.6^{\circ}\end{array}$ & $\begin{array}{l}0.4 \\
0.8\end{array}$ & 14.2 & 5.3 & $\begin{array}{l}13.0 \\
10.4\end{array}$ & $\begin{array}{l}4.1 \\
1.5\end{array}$ & 12.6 & 3.9 & 14.6 & 5.9 & 16.1 & 7.5 & 14.3 & 6.3 & 16.5 & 8.9 & 15.0 & 7.1 \\
\hline 7 & 10.2 & 1.4 & $1+t .0$ & 5.1 & $\begin{array}{l}10.4 \\
10.4\end{array}$ & $\begin{array}{l}1.5 \\
1.5\end{array}$ & $\begin{array}{l}11.6 \\
11.2\end{array}$ & $\begin{array}{l}3.9 \\
2.5\end{array}$ & 14.2 & 5.5 & 15.8 & 7.2 & 13.2 & 5.2 & $\begin{array}{l}17.4 \\
17.6\end{array}$ & $\begin{array}{r}9.5 \\
10.0\end{array}$ & 16.7 & S.S \\
\hline 8 & 12.4 & 3.6 & $\begin{array}{l}10.0 \\
11.0\end{array}$ & $\begin{array}{l}1.1 \\
2.1\end{array}$ & $\begin{array}{l}111.2 \\
10.6\end{array}$ & $\begin{array}{l}1.3 \\
1.7\end{array}$ & $\begin{array}{l}11.0 \\
11.2\end{array}$ & $\begin{array}{l}2.3 \\
2.5\end{array}$ & 12.8 & \pm .1 & 15.2 & 6.7 & 13.6 & 5.15 & 17.4 & 9.8 & 17.8 & 9.9 \\
\hline 9 & 15.0 & 6.2 & 12.0 & 3.1 & 11.0 & 2.1 & 11.4 & 2.7 & 12.4 & 3.7 & 14.2 & 5.7 & 14.3 & 6.3 & 16.8 & @.1 & 18.4 & 10.5 \\
\hline 10 & $1 \leqslant .5$ & 9.7 & 15.0 & 6.1 & 12.4 & 3.6 & 13.6 & 4.9 & 13.0 & 4.3 & 13.4 & 4.9 & 15.0 & $?$ & 15.5 & 7.8 & 18.5 & 10.5 \\
\hline 11 & 21.0 & 12.2 & ] 8.0 & 9.1 & 15.0 & 13.2 & 15.0 & 6.3 & 14.0 & 5.3 & 13.0 & 4.6 & 14.0 & 6.2 & 14.5 & (j.s & $1(i, ?$ & 8.9 \\
\hline Mirln't & $\ldots$ & $\ldots$ & 211.4 & 11.5 & 18.0 & 9.2 & $\ldots$ & ... & 15.2 & 6.5 & 13.11 & 4.13 & 13.4 & 5.6 & 14.3 & ij. (j & $15.1 ;$ & 7.6 \\
\hline
\end{tabular}

Jun 12. Correction - 8.8 The recort on this day is defective, the timos being unrertain.

$\begin{array}{llll}\text { " } & 13 . & \text { " } & -8.9 \\ \text { " } 15 . & \text { " } & -8.7 \\ \text { " } 17 . & \text { " } & -8.6 \\ 11 . & \text { " } & -7.6\end{array}$
June 14. Correction -5.9

" $16 . \quad$ " $\quad$ " $\quad-8.7$

"21\%. " 
Series III.-'Midal Orservations Frow $\lambda$ pril 20 to $A$ uqust $3,1854$.

IIourly observations on the pulley-gange. Adopted reacling of mean level 7.0, expressed in units of the scale. Increasing numbers indieate rise of water.

\section{June, 1854.}

\begin{tabular}{|c|c|c|c|c|c|c|c|c|c|c|c|c|c|c|c|c|c|c|}
\hline $\begin{array}{l}\text { Mern } \\
\text { solar } \\
\text { bour. }\end{array}$ & 21 st. & $\begin{array}{l}\text { Ref. } \\
\text { olss. }\end{array}$ & $22 \mathrm{~d}$ & $\begin{array}{l}\text { Ref. } \\
\text { obs. }\end{array}$ & 230. & $\begin{array}{l}\text { Ref. } \\
\text { obs. }\end{array}$ & $24 t h$. & $\begin{array}{l}\text { Ref. } \\
\text { obs. }\end{array}$ & 25 th. & $\begin{array}{l}\text { Ref. } \\
\text { obs. }\end{array}$ & 26 th. & $\begin{array}{l}\text { Ref. } \\
\text { olss. }\end{array}$ & 27 th. & $\begin{array}{l}\text { Ref. } \\
\text { olos. }\end{array}$ & $29 \mathrm{th}$. & $\begin{array}{l}\text { Ref. } \\
\text { olsa. }\end{array}$ & 29th. & $\begin{array}{l}\text { Ref. } \\
\text { obs. }\end{array}$ \\
\hline 1 & $\begin{array}{l}14.5 \\
14.2\end{array}$ & $\begin{array}{l}6.5 \\
6.2\end{array}$ & 15.2 & 6.6 & 16.5 & 7.0 & $\begin{array}{l}21.0 \\
211.5\end{array}$ & $\begin{array}{l}10.6 \\
10.1\end{array}$ & 19.0 & 8.3 & $\begin{array}{l}20.4 \\
22.4\end{array}$ & $\begin{array}{l}12.1 \\
12.1\end{array}$ & 211.2 & 10.1 & 21.4 & $11 . t$ & $\begin{array}{l}21.4 \\
21.4 \\
21.4\end{array}$ & $\begin{array}{l}11.4 \\
11.4 \\
11.4\end{array}$ \\
\hline 2 & $\begin{array}{l}13.8 \\
13.8\end{array}$ & $\begin{array}{l}5.8 \\
5.8\end{array}$ & 13.9 & 5.3 & 14.7 & 5.2 & 18.0 & 7.6 & 17.0 & 6.3 & 211.0 & 9.7 & 20.2 & 10.1 & 21.4 & 11.4 & 21.4 & 11.4 \\
\hline 3 & 13.5 & 5.5 & 11.5 & $\begin{array}{l}2.9 \\
+.7\end{array}$ & 13.8 & 4.3 & 15.8 & 5.3 & 16.0 & 5.3 & $17.1^{\circ}$ & 7.3 & $1 S .5$ & 8.4 & 19.1 & 9.1 & 20.0 & 10.0 \\
\hline 4 & 14.9 & 5.9 & -.. & .. & 13.2 & $\begin{array}{l}3.0 \\
3.6\end{array}$ & $\begin{array}{l}10.0 \\
14.2\end{array}$ & $\begin{array}{l}4.0 \\
3.6\end{array}$ & 14.1 & 3.5 & 15.0 & 4.8 & 17.0 & 6.9 & 16.3 & 6.3 & 18.0 & 8.0 \\
\hline 5 & 14.4 & 6.3 & $\cdots$ & $\cdots$ & 14.11 & 4.3 & 14.2 & 3.6 & 13.0 & 2.4 & 12.2 & 2. 0 & 12.0 & 1.9 & 15.3 & 5.3 & 16.6 & 6.6 \\
\hline 6 & 15.7 & 7.6 & ... & $\ldots$ & 14.6 & 4.9 & 15.1 & 4.4 & 13.4 & 2.5 & 13.4 & 3.2 & 12.2 & 2.1 & 14.4 & 4.4 & 15.5 & 5.5 \\
\hline 7 & 16.3 & 8.2 & $\cdots$ & $\cdots$ & 15.0 & 5.2 & 15.3 & 4.6 & 14.0 & 3.4 & 13.8 & 3.7 & 12.5 & 2.7 & 13.8 & 3.9 & 13.9 & 3.9 \\
\hline 8 & $\begin{array}{l}17.2 \\
17.2\end{array}$ & $\begin{array}{l}9.1 \\
9.0\end{array}$ & 16.7 & 7.6 & 15.6 & 5.8 & 15.5 & 4.7 & 14.5 & 3.9 & 14.4 & 4.3 & 13.1 & 3.3 & $\begin{array}{l}13.2 \\
13.5\end{array}$ & $\begin{array}{l}3.2 \\
3.5\end{array}$ & $\begin{array}{l}13.6 \\
13.9\end{array}$ & $\begin{array}{l}3.6 \\
3.9\end{array}$ \\
\hline 9 & $\begin{array}{l}17.6 \\
17.2\end{array}$ & $\begin{array}{l}9.4 \\
0.0\end{array}$ & $\begin{array}{l}17.6 \\
13.0\end{array}$ & $\begin{array}{l}8.5 \\
9.9\end{array}$ & 18.4 & 8.5 & 16.4 & 5.6 & 16.2 & 5.6 & 16.0 & 5.9 & 14.6 & 4.5 & 14.0 & 4.0 & 14.4 & 4.4 \\
\hline 10 & 16.2 & 8.0 & 18.5 & 9.4 & 18.4 & 8.5 & 17.5 & 6.7 & 17.0 & 6.4 & 17.2 & 7.1 & 16.4 & 6.3 & 15.5 & 5.5 & 16.2 & 6.2 \\
\hline 11 & 15.8 & 7.6 & 17.5 & 8.4 & 18.1 & 8.1 & 18.3 & 7.5 & $\begin{array}{l}18.1 \\
15.8\end{array}$ & $\begin{array}{l}7.5 \\
8.2\end{array}$ & 18.8 & 8.7 & 18.0 & 7.9 & 17.8 & 7.8 & 18.0 & 8.0 \\
\hline Noon & 14.6 & 6.1 & 16.5 & 7.4 & 17.8 & 7.8 & $\begin{array}{l}15.9 \\
18.9\end{array}$ & $\begin{array}{l}8.1 \\
8.1\end{array}$ & 18.9 & 8.3 & 19.8 & 9.7 & $\begin{array}{l}19.4 \\
13.9\end{array}$ & $\begin{array}{l}9.3 \\
9.5\end{array}$ & $\begin{array}{l}19.1 \\
19.2\end{array}$ & $\begin{array}{l}9.1 \\
9.2\end{array}$ & 18.4 & 8.4 \\
\hline 1 & 14.0 & 5.5 & 16.0 & 6.9 & 16.0 & 6.0 & 14.5 & 7.7 & 18.2 & 7.6 & $\begin{array}{l}20.0 \\
19.0\end{array}$ & $\begin{array}{l}9.9 \\
8.9\end{array}$ & 19.0 & 8.9 & $\begin{array}{l}19.2 \\
19.2\end{array}$ & $\begin{array}{l}9.2 \\
9.2\end{array}$ & $\begin{array}{l}19.0 \\
19.1\end{array}$ & $\begin{array}{l}9.0 \\
9.1\end{array}$ \\
\hline 2 & $\begin{array}{l}13.0 \\
12.4\end{array}$ & $\begin{array}{l}4.8 \\
4.2\end{array}$ & 15.4 & 6.3 & 15.5 & 5.5 & 15.9 & 5.1 & 16.5 & 5.9 & 18.2 & 8.1 & 17.6 & 7.5 & 15.6 & 8.6 & $\begin{array}{l}13.1 \\
18.8\end{array}$ & $\begin{array}{l}3.1 \\
8.5\end{array}$ \\
\hline 3 & 11.2 & $\begin{array}{l}3.0 \\
3.7\end{array}$ & 14.4 & 5.3 & 14.0 & 3.9 & 14.7 & 3.9 & 15.7 & 5.1 & 16.4 & 6.3 & 16.8 & 6.7 & 17.5 & 7.5 & 17.5 & 7.5 \\
\hline 4 & 13.9 & 5.6 & $\begin{array}{l}13.2 \\
12.8\end{array}$ & $\begin{array}{l}4.1 \\
3.7\end{array}$ & $\begin{array}{l}13.6 \\
13.5\end{array}$ & $\begin{array}{l}3.5 \\
3.3\end{array}$ & 13.9 & 3.1 & $\ldots$ & - - & 15.0 & 4.9 & 16.0 & 5.9 & 17.2 & 7.2 & 16.4 & 6.4 \\
\hline 5 & 14.6 & 6.3 & $\begin{array}{l}12.5 \\
13.0\end{array}$ & $\begin{array}{l}3.3 \\
3.8\end{array}$ & $\begin{array}{l}13.2 \\
13.2\end{array}$ & $\begin{array}{l}3.01 \\
2.9\end{array}$ & $\begin{array}{l}13.2 \\
12.8\end{array}$ & $\begin{array}{l}2.4 \\
2.0\end{array}$ & $\ldots$ & $\ldots$ & 14.0 & 3.9 & 14.8 & 4.7 & 16.8 & 6.5 & 13.4 & 3.4 \\
\hline 6 & 16.5 & 8.2 & 14.6 & 5.4 & $\begin{array}{l}13.2 \\
14.2\end{array}$ & $\begin{array}{l}2.9 \\
3.9\end{array}$ & 13.5 & 2.7 & 13.7 & 3.2 & $\begin{array}{l}13.3 \\
12.5\end{array}$ & $\begin{array}{l}3.2 \\
2.1\end{array}$ & 13.5 & 3.5 & 15.1 & 5.1 & $\begin{array}{l}12.1 \\
12.0\end{array}$ & $\begin{array}{l}2.1 \\
2.0\end{array}$ \\
\hline 7 & 18.4 & 10.0 & 17.2 & 7.9 & 15.0 & 4.7 & 15.0 & 4.2 & 13.6 & 3.1 & 13.0 & 2.9 & $\begin{array}{l}13.8 \\
13.4\end{array}$ & $\begin{array}{l}3.8 \\
3.1\end{array}$ & $\begin{array}{l}12.8 \\
12.5\end{array}$ & $\begin{array}{l}2.8 \\
2.5\end{array}$ & 12.6 & 2.6 \\
\hline 8 & 19.5 & 11.1 & 18.0 & 5.7 & 16.0 & 5.7 & 17.0 & 6.2 & 15.0 & 4.5 & 15.0 & 4.9 & 13.2 & 3.2 & 13.1 & 3.1 & 13.5 & 3.6 \\
\hline 9 & $\begin{array}{l}20.0 \\
21.5\end{array}$ & $\begin{array}{l}11.6 \\
13.0\end{array}$ & $\begin{array}{l}19.0 \\
19.5\end{array}$ & $\begin{array}{r}9.6 \\
10.1\end{array}$ & 19.0 & 8.7 & 19.0 & 8.2 & 18.0 & 7.5 & 19.4 & 9.3 & 15.0 & 5.0 & 15.1 & 5.1 & 15.2 & 5.3 \\
\hline 10 & 20.5 & 12.0 & $\begin{array}{l}20.0 \\
20.0\end{array}$ & $\begin{array}{l}10.6 \\
10.6\end{array}$ & 21.0 & 10.6 & $\begin{array}{l}21.0 \\
21.5\end{array}$ & $\begin{array}{l}10.2 \\
10.8\end{array}$ & 20.2 & 9.8 & 22.2 & 12.1 & 17.5 & 7.5 & 17.2 & 7.2 & 16.7 & 6.8 \\
\hline 11 & 18.5 & 10.0 & 19.8 & 10.3 & $\begin{array}{l}21.4 \\
21.4\end{array}$ & $\begin{array}{l}11.0 \\
11.0\end{array}$ & $\begin{array}{l}22.0 \\
21.8\end{array}$ & $\begin{array}{l}11.3 \\
11.1\end{array}$ & 21.5 & 11.1 & 22.4 & 12.3 & 20.0 & 10.0 & 19.2 & 9.2 & 17.8 & 8.0 \\
\hline Miru't & 14.1 & 5.6 & 19.4 & 9.9 & 21.4 & 11.0 & 20.6 & 9.3 & 22.0 & 11.6 & ... & $\ldots$ & 21.4 & 11.4 & 21.3 & 11.3 & 19.5 & 9.7 \\
\hline
\end{tabular}

$$
\begin{array}{cccc}
\text { June 21. } & \text { Correction }-8.2 \\
\text { " } 23 . & \text { " } & -10.0 \\
\text { " } & 25 . & \text { " } & -10.6 \\
\text { " } & 27 . & \text { " } & -10.1 \\
\text { " } 29 . & \text { " } & -10.0 \\
\text { " } & 29 . & \text { " } & -10.0
\end{array}
$$

June 22. Correction -9.1

" $24 . \quad$ " $\quad-10.8$

" $26.4 \quad-10.1$

Some doubt alont the time record in the afternoon. 
Series III.-Tidal Observations from April 20 to August 3, 1854.

Hourly observations on the pulley-gange. Adopted reading of mean lerel 7.0, expressed in units of the seale. Increasing numbers indicate rise of water.

\begin{tabular}{|c|c|c|c|c|c|c|c|c|c|c|c|c|c|c|c|c|c|c|}
\hline \multicolumn{3}{|c|}{ June, 1854.} & \multicolumn{16}{|c|}{ July, 1854.} \\
\hline $\begin{array}{l}\text { Mean } \\
\text { solar } \\
\text { bour. }\end{array}$ & $30 \mathrm{tb}$. & $\begin{array}{l}\text { Ref- } \\
\text { obs. }\end{array}$ & 1st. & $\begin{array}{l}\text { Ref. } \\
\text { obs. }\end{array}$ & $2 \mathrm{~d}$. & $\begin{array}{l}\text { Ref. } \\
\text { obs. }\end{array}$ & $3 \mathrm{~d}$. & $\begin{array}{l}\text { Ref. } \\
\text { obs. }\end{array}$ & 4tb. & $\begin{array}{l}\text { Ref. } \\
\text { obs. }\end{array}$ & 5th. & $\begin{array}{l}\text { Ref. } \\
\text { olss. }\end{array}$ & 6th. & $\begin{array}{l}\text { Ref. } \\
\text { obs. }\end{array}$ & 7 th. & $\begin{array}{l}\text { Ref. } \\
\text { obs. }\end{array}$ & 9 th. & $\begin{array}{l}\text { Ref. } \\
\text { ubs. }\end{array}$ \\
\hline 1 & 19.0 & 9.2 & 18.5 & 9.0 & 20.6 & 11.0 & 14.5 & 5.9 & 16.3 & 7.6 & 15.2 & 6.5 & $\begin{array}{l}15.7 \\
15.0 \\
15.0\end{array}$ & $\begin{array}{l}6.7 \\
6.0 \\
6.0\end{array}$ & 17.0 & 7.8 & 15.5 & 8.3 \\
\hline 2 & 20.8 & 11.0 & 19.8 & 10.3 & 21.0 & 11.3 & 16.3 & 7.7 & 16.9 & 8.2 & 15.6 & 6.9 & $\begin{array}{l}15.0 \\
15.0\end{array}$ & $\begin{array}{l}6.0 \\
6.0\end{array}$ & 15.8 & 5.6 & 13.0 & 5.8 \\
\hline \multirow[t]{2}{*}{3} & 21.0 & 11.2 & 20.7 & 11.2 & 21.4 & 11.7 & 17.3 & 8.7 & 17.4 & 8.7 & 16.8 & 8.1 & $\begin{array}{l}15.0 \\
15.0\end{array}$ & $\begin{array}{l}6.0 \\
6.0\end{array}$ & $\begin{array}{l}15.8 \\
15.8\end{array}$ & $\begin{array}{l}5.6 \\
5.6\end{array}$ & 11.0 & 3.9 \\
\hline & 22.0 & 12.3 & 20.9 & 11.4 & & & 17.6 & 8.9 & & & & & 15.6 & 6.6 & 15.8 & 5.6 & & \\
\hline 4 & 20.4 & 10.7 & $\begin{array}{l}21.0 \\
21.0\end{array}$ & $\begin{array}{l}11.5 \\
11.6\end{array}$ & 21.4 & 11.7 & $\begin{array}{l}17.8 \\
17.8\end{array}$ & $\begin{array}{l}9.1 \\
9.1\end{array}$ & 18.6 & 9.9 & 17.3 & 8.6 & 15.6 & 6.6 & $\begin{array}{l}15.8 \\
16.0\end{array}$ & $\begin{array}{l}5.6 \\
5.8\end{array}$ & $\begin{array}{l}9.8 \\
9.5\end{array}$ & $\begin{array}{l}2.7 \\
2.4\end{array}$ \\
\hline 5 & 19.8 & 10.1 & $\begin{array}{l}21.0 \\
20.7\end{array}$ & $\begin{array}{l}11.6 \\
11.3\end{array}$ & 17.6 & 7.8 & 17.5 & 8.8 & 18.6 & 10.0 & 18.4 & 9.7 & 17.0 & 8.0 & 16.4 & 6.2 & $\begin{array}{l}9.5 \\
9.5\end{array}$ & $\begin{array}{l}2.4 \\
2.5\end{array}$ \\
\hline 6 & 18.0 & 8.4 & 19.5 & 10.1 & 15.7 & 5.9 & 17.0 & 8.2 & \begin{tabular}{|l|}
18.6 \\
18.8
\end{tabular} & $\begin{array}{l}10.0 \\
10.2\end{array}$ & $\begin{array}{l}18.6 \\
18.8\end{array}$ & $\begin{array}{r}9.8 \\
10.0\end{array}$ & 17.6 & 8.5 & 17.2 & 7.0 & 9.8 & 2.8 \\
\hline 7 & 17.0 & 7.4 & 17.1 & 7.7 & 14.4 & 4.6 & 16.3 & 7.5 & 18.8 & 10.2 & 18.9 & 10.1 & 18.2 & 9.1 & 18.8 & 8.6 & 10.5 & 3.5 \\
\hline \multirow[t]{2}{*}{ s } & 15.0 & 5.4 & 15.7 & 6.3 & 12.7 & +.5 & 757 & 60 & 18.4 & 9.8 & 18.4 & 9.6 & 18.5 & 9.4 & 19.0 & 8.8 & & -9 \\
\hline & 14.0 & 4.4 & & & & 4.5 & 10.4 & 6.9 & 17.6 & 9.0 & 18.4 & 9.6 & $\begin{array}{l}17.5 \\
17.8\end{array}$ & 8.7 & $\begin{array}{l}19.4 \\
19.1\end{array}$ & 8.9 & 12.4 & 5.4 \\
\hline 9 & 13.5 & 3.9 & $\begin{array}{l}13.5 \\
70.2\end{array}$ & 4.1 & 12.0 & 3.8 & 14.0 & 5.2 & $|15.0|$ & 6.4 & 18.2 & 9.4 & 17.0 & 7.8 & 18.5 & 8.3 & 13.8 & 6.8 \\
\hline \multirow[t]{2}{*}{10} & 16.1 & 6.5 & $\begin{array}{l}12.2 \\
12.0\end{array}$ & $\begin{array}{l}2.8 \\
2.6\end{array}$ & $\left|\begin{array}{l}11.7 \\
11.4\end{array}\right|$ & $\begin{array}{l}3.5 \\
2.2\end{array}$ & \begin{tabular}{|}
14.0 \\
13.6
\end{tabular} & $\begin{array}{l}5.2 \\
4.8\end{array}$ & 14.0 & 5.4 & 17.0 & 8.2 & 169 & 70 & 16.8 & 66 & 154 & \\
\hline & & & 12.5 & 3.1 & 11.4 & 2.2 & 13.6 & 4.8 & 13.6 & 5.0 & 10.0 & 8.2 & 10.5 & 1.0 & 10.8 & 0.0 & $\begin{array}{l}15.4 \\
15.5\end{array}$ & $\begin{array}{l}8.4 \\
8.5\end{array}$ \\
\hline 11 & 18.7 & 9.1 & 13.0 & 3.6 & 11.4 & 2.2 & $13.6^{\circ}$ & 4.8 & 13.0 & 4.4 & 15.0 & 6.2 & 15.9 & 6.7 & 15.5 & 5.3 & 15.5 & 8.5 \\
\hline \multirow[t]{2}{*}{ Noon } & \begin{tabular}{|l|}
18.9 \\
19.0
\end{tabular} & $\begin{array}{l}9.3 \\
9.4\end{array}$ & 15.4 & 6.0 & $\begin{array}{l}11.4 \\
11.9\end{array}$ & $\begin{array}{l}2.2 \\
3.7\end{array}$ & $\begin{array}{l}15.0 \\
16.5\end{array}$ & $\begin{array}{l}6.2 \\
7.7\end{array}$ & $\begin{array}{l}13.1 \\
13.1\end{array}$ & $\begin{array}{l}4.4 \\
4.5\end{array}$ & 14.0 & 5.2 & 14.0 & 4.8 & 15.5 & 5.3 & $\begin{array}{l}149 \\
14.3\end{array}$ & $\begin{array}{l}7.9 \\
7.3\end{array}$ \\
\hline & 19.0 & 9.4 & & & & & & & & & 13.3 & 4.5 & 13.1 & 3.9 & & & & 7.3 \\
\hline 1 & 18.8 & 9.2 & 17.2 & 7.8 & 12.2 & 3.9 & 18.3 & $?$ & 13.2 & 4.6 & $\begin{array}{l}13.1 \\
13.1\end{array}$ & $\begin{array}{l}4.3 \\
4.3\end{array}$ & 13.1 & 3.9 & 14.4 & 4.2 & 13.2 & 6.2 \\
\hline 2 & 17.0 & 7.4 & 18.1 & 8.7 & 13.4 & 5.1 & 16.2 & 7.4 & 14.0 & 5.4 & 13.1 & 4.3 & 13.1 & 3.9 & 13.3 & 3.1 & 11.2 & 4.3 \\
\hline 3 & 15.8 & 6.2 & 18.3 & 8.9 & 14.4 & 6.1 & 17.2 & 8.4 & 15.3 & 6.7 & $\begin{array}{l}13.2 \\
13.4\end{array}$ & $\begin{array}{l}4.4 \\
4.6\end{array}$ & $\begin{array}{l}13.1 \\
13.4\end{array}$ & $\begin{array}{l}3.9 \\
4.2\end{array}$ & $\begin{array}{l}13.2 \\
13.2\end{array}$ & $\begin{array}{l}3.0 \\
3.0\end{array}$ & 9.1 & 2.2 \\
\hline 4 & 15.0 & 5.4 & 18.3 & 8.9 & 15.2 & 6.9 & 18.2 & 9.4 & 17.2 & 8.6 & 16.1 & 7.3 & 14.3 & 5.1 & $\begin{array}{l}13.2 \\
13.2\end{array}$ & $\begin{array}{l}3.0 \\
3.0\end{array}$ & $\begin{array}{l}8.3 \\
8.0\end{array}$ & 1.5 \\
\hline \multirow{2}{*}{5} & & & & & & & 18.4 & 9.6 & & & & & & & 13.7 & 3.5 & 8.0 & 1.3 \\
\hline & 14.0 & 4.4 & $\begin{array}{l}18.7 \\
17.8\end{array}$ & $\begin{array}{l}8.3 \\
8.4\end{array}$ & $\begin{array}{l}15.6 \\
15.7\end{array}$ & $\begin{array}{l}7.2 \\
7.3\end{array}$ & \begin{tabular}{|l|}
18.6 \\
18.6
\end{tabular} & $\begin{array}{l}9.8 \\
9.8\end{array}$ & 17.4 & 8.7 & 17.5 & 8.7 & 17.3 & 8.1 & \begin{tabular}{|l|}
14.2 \\
15.3
\end{tabular} & 4.0 & 8.0 & 1.3 \\
\hline \multirow[t]{2}{*}{6} & 13.5 & 3.9 & 17.3 & 7.8 & 15.3 & 6.9 & 18.6 & 9.8 & 18.0 & 9.3 & 18.0 & 9.2 & 18.3 & 9.1 & & 5.1 & $\begin{array}{l}8.0 \\
9.0\end{array}$ & $\begin{array}{l}2.0 \\
2.4\end{array}$ \\
\hline & 13.4 & 3.8 & 16.2 & 6.7 & 14.6 & 6.2 & \begin{tabular}{|l|}
18.3 \\
18.0
\end{tabular} & $\begin{array}{l}9.5 \\
9.2\end{array}$ & $\begin{array}{l}18.5 \\
18.5\end{array}$ & $\begin{array}{l}9.8 \\
9.8\end{array}$ & $\begin{array}{l}18.7 \\
19.4\end{array}$ & $\begin{array}{r}9.9 \\
10.6\end{array}$ & 19.5 & 10.3 & 16.6 & 6.4 & & 45 \\
\hline & 10.4 & 0.0 & & & & & & & 18.5 & 9.8 & 19.4 & 10.5 & 19.9 & 10.7 & 17.4 & 0.4 & 11.0 & 4.5 \\
\hline 8 & 13.4 & 3.8 & 14.7 & 5.2 & 14.0 & 5.5 & 17.5 & 8.7 & 18.1 & 9.3 & 19.4 & 10.5 & 20.4 & 11.2 & & 7.2 & 13.4 & 6.9 \\
\hline .9 & 14.1 & 4.5 & 14.5 & 5.0 & 14.0 & 5.5 & 16.7 & 7.9 & 17.4 & 8.7 & $\left.\begin{array}{l}19.4 \\
19.4\end{array}\right]$ & 10.5 & 20.4 & 11.2 & 18.0 & 7.8 & 15.9 & 9.5 \\
\hline 10 & 15.4 & 5.8 & 17.5 & 8.0 & 12.8 & 4.3 & 15.8 & 7.0 & 16.2 & 7.5 & 19.2 & 10.3 & 20.4 & 11.2 & 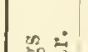 & & 18 ? & 110 \\
\hline & & & & & 12.4 & 3.9 & $\begin{array}{l}10.8 \\
15.6\end{array}$ & 6.9 & & 1.0 & 18.7 & 9.8 & 20.4 & 10.9 & $\Xi \Xi$ & & 18.2 & 11.9 \\
\hline 11 & 16.0 & 6.4 & 18.9 & 9.3 & 12.3 & 3.8 & 15.6 & 6.9 & 15.8 & 7.1 & 17.2 & 8.3 & 19.7 & 10.5 & 党 & & 18.6 & 12.3 \\
\hline Wisln't & 15.7 & 7.1 & 19.7 & 10.1 & $\begin{array}{l}12.3 \\
12.3\end{array}$ & $\begin{array}{l}3.8 \\
3.8\end{array}$ & $\begin{array}{l}15.6 \\
15.7\end{array}$ & $\begin{array}{l}6.3 \\
7.0\end{array}$ & 15.2 & 6.5 & 16.0 & 7.1 & 17.1 & 7.9 & 蛋 & & $\begin{array}{l}18.7 \\
18.7\end{array}$ & $\begin{array}{l}12.5 \\
12.5\end{array}$ \\
\hline & & & - & & & & & & & & & & & & & & & \\
\hline Juni & 10311. & Correc & ction - & $-9.4 ;$ & & & & & & & & & July 1. & - Corre & ection & -9.4 & & \\
\hline July & y 2. & " & & -9.81 & before 8 & $8 \Lambda . M$ & i., and & $1-8$. & 2 after & $8 \mathrm{~A}$. & M. & & " 3. & . $\quad$ ، & & -8.8 & & \\
\hline " & 4. & " & & -8.6 & & & & & & & & & “5. & “ & & -8.8 & & \\
\hline " & if. & " & & -9.2 & & & & & & & & & & & & & & \\
\hline " & 7. & " & & -9.2 & Tide & registe & er out & $t$ of 0 & orider a & at 20 & b'cloek, & , chas & nged $i$ & index 1 & 1 foot; & ; corr & ection & after \\
\hline " & 8. & The re & & sa anpe & ear irre & egular. & - Corr & rectio & "n & $100 \mathrm{n}-$ & $-7.11, \mathrm{at}$ & at misl & liniglit & - & & & A. A. & 10.2. \\
\hline
\end{tabular}


Series III.-Tidal Oisservations from A pril 20 to 1 ugust 3, 1854.

IIourly observations on the pulley-gauge. Adopted reading of mean level 7.0, expressed in units of the scale. Inereasing numbers indieate rise of water.

\section{July, 1854.}

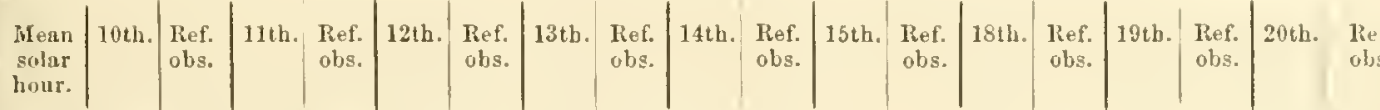

\begin{tabular}{l|l|l|l|l|l|l|l|l|l|l|l|l|l|l|l|l|l}
18.4 & 12.3 & 19.3 & 13.4 & 19.3 & 13.3 & 19.5 & 13.5 & 19.0 & 13.0 & 18.1 & $?$ & $\ldots$ & $\ldots$. & $\ldots$. & $\ldots$. & 20.5 & 6.1
\end{tabular}

\begin{tabular}{|l|l|l|l|l|l|l|l|l|l|l|l|l|}
10.6 & 10.7 & 19.0 & 13.1 & 19.3 & 13.3 & 19.0 & 13.6 & 17.0 & 11.0 & 18.1 & ? \\
\hline
\end{tabular}

\begin{tabular}{l|l|l|l|l|l|l|l|l|l|l}
3 & 12.5 & 6.5 & 14.0 & 8.0 & 16.5 & 10.5 & 17.8 & 11.8 & 18.4 & 12.4
\end{tabular}

\begin{tabular}{r|r|r|r|r|r|r|r|r|r|r}
10.0 & 4.0 & 12.1 & 6.1 & 12.0 & 6.0 & 16.3 & 10.3 & 17.3 & 11.3 \\
9.0 & 3.0 & & & & &
\end{tabular}

$9.0 \quad 3.0$

8.0

$15.0 \quad 9.0$

\begin{tabular}{l|l|l|l|l|l}
.0 & 2.0 & 7.8 & 1.5 & 10.2 & 4.2
\end{tabular}

$7.1 \quad 1.1$

\begin{tabular}{l|l|l|l|lll|l|l}
9.0 & 3.0 & 7.8 & 1.5 & 7.0 & 1.0 & 7.5 & 1.5 \\
9.4 & $?$ & 8.5 & 2.5 & 7.3 & 1.3 & 7.0 & 1.0
\end{tabular}

\begin{tabular}{ll|l|lllll|l|l}
9.4 & $\cdots$ & 5.5 & 2.5 & 7.3 & 1.3 & 7.0 & 1.0 \\
\hline .6 & $-\cdots$ & 9.0 & 3.0 & 8.2 & 2.2 & 7.0 & 1.0
\end{tabular}

\begin{tabular}{l|l|l|l|l|l|l|l|}
7.2 & --- & 11.5 & 0.5 & 10.5 & 4.5 & 8.0 & 2.0
\end{tabular}

\begin{tabular}{lllllllll|l|l}
0 & 10.3 & 4.5 & 14.0 & 8.0 & 13.0 & 7.0 & 10.2 & 4.2
\end{tabular}

\begin{tabular}{l|l}
7.8 & 1.8 \\
7.9 & 1.9
\end{tabular}

$\begin{array}{lllllllllll}11 & 19.1 & 0.3 & 15.6 & 9.6 & 15.4 & 9.4 & 12.6 & 0.0 & 0.3 & 3.3\end{array}$

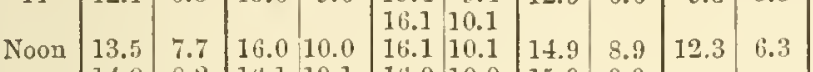

\begin{tabular}{llll|l|l|l|l|l}
14.0 & 8.2 & 16.1 & 10.1 & 16.0 & 10.0 & 15.9 & 9.9
\end{tabular}

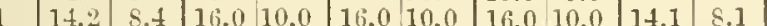

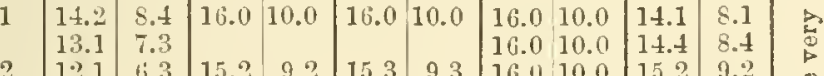

\begin{tabular}{llllllll|l|l|l|l|}
12.1 & 6.3 & 15.2 & 9.2 & 15.3 & 9.3 & 16.0 & 10.0 & 15.2 & 9.2 \\
\hline
\end{tabular}

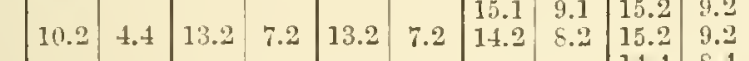

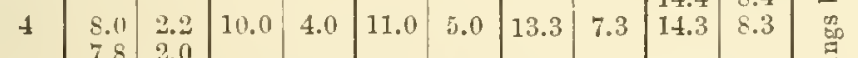

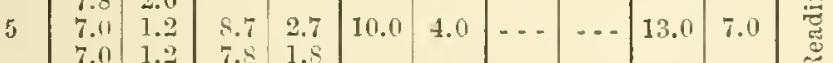

\begin{tabular}{l|l|l|l|l|l|l|l|l|l|l|l}
6 & 7.6 & 1.7 & 7.2 & 1.2 & 8.7 & 2.7 & $\ldots$ & $\ldots$ & 12.5 & 6.5 &
\end{tabular}

\begin{tabular}{|l|l|l|l|l|l|l|l|}
10.1 & 4.2 & 7.5 & 1.5 & 7.2 & 1.2 & 9.0 & 3.0 \\
& & & & 7.2 & 1.2 & 8.7 & 2.7 \\
\hline
\end{tabular}

\begin{tabular}{l|l|l|l|l|l|l|l|l}
8 & 13.1 & 7.2 & 9.0 & 3.0 & 7.6 & 1.6 & 8.4 & 2.4
\end{tabular}

\begin{tabular}{l|lll|l|l|l|l|l|l|l}
9 & 15.0 & 9.1 & 10.5 & 4.5 & 9.2 & 3.2 & $\ldots$ & $\ldots$ & 8.5 & 2.5
\end{tabular}

\begin{tabular}{l|l|l|l|l|l|l|l|l|l|l|l}
10 & 17.0 & 11.1 & 13.9 & 7.9 & 12.2 & 6.2 & -- & $\ldots$ & 9.9 & 2.9 \\
9.0 & 3.0
\end{tabular}

\begin{tabular}{l|l|l|l|l|l|l|l|l|l|l}
11 & 18.6 & 12.7 & 16.8 & 10.8 & 10.8 & 10.8 & -- & $\ldots$ & 11.5 & 5.5
\end{tabular}

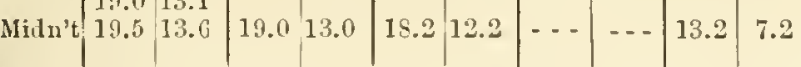

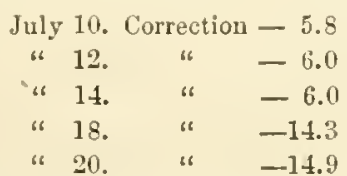

July 10. Correction - 5.8

" 14.060

" $20 . \quad$ " $\quad-14.9$
July 11. Correction - 6.0

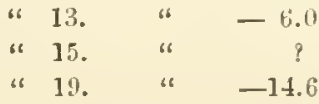


Sertes III.-Tidal Ogservations from A pril 20 to Auguat 3, 1854.

Honrly olserrations on the pulley-gange. Adopted reading of mean level 7.0, expressed in units of the scale. Increasing numbers indicate rise of water.

\begin{tabular}{|c|c|c|c|c|c|c|c|c|c|c|c|c|c|c|}
\hline \multirow[b]{2}{*}{$\begin{array}{l}\text { Mean } \\
\text { solar } \\
\text { hour. }\end{array}$} & \multicolumn{8}{|c|}{ July; 1854.} & \multicolumn{6}{|c|}{ August, 1854.} \\
\hline & 2 Sth. & $\begin{array}{l}\text { Ref. } \\
\text { obs. }\end{array}$ & 29th. & $\begin{array}{l}\text { Ref. } \\
\text { obs. }\end{array}$ & 30 th. & $\begin{array}{l}\text { Ref. } \\
\text { obs. }\end{array}$ & 31st. & $\begin{array}{l}\text { Ref. } \\
\text { obs. }\end{array}$ & 1st. & $\begin{array}{l}\text { Ref. } \\
\text { obs. }\end{array}$ & $2 \mathrm{~d}$. & $\begin{array}{l}\text { Ref. } \\
\text { obs. }\end{array}$ & $3 d$. & $\begin{array}{l}\text { Ret. } \\
\text { obs. }\end{array}$ \\
\hline 1 & & & 10.6 & 10.1 & 10.6 & 10.5 & 8.3 & 8.3 & 8.0 & 8.0 & 6.8 & 7.0 & 5.3 & 5.7 \\
\hline 2 & E & & 11.3 & 10.8 & $\begin{array}{l}11.2 \\
11.2\end{array}$ & $\begin{array}{l}11.1 \\
11.1\end{array}$ & 8.6 & 8.6 & 8.4 & 5.4 & 8.0 & 8.2 & $\begin{array}{l}5.5 \\
6.0\end{array}$ & $\begin{array}{l}5.9 \\
6.4\end{array}$ \\
\hline & $\underline{E}$ & & 12.3 & 11.8 & 11.2 & 11.1 & & & 9.1 & 9.1 & & & & \\
\hline 3 & $\geq$ & & 12.5 & 12.0 & 11.2 & 11.1 & 9.3 & 9.3 & 9.3 & 9.3 & 8.7 & 8.9 & 6.5 & 6.9 \\
\hline 4 & $\vec{E}$ & & $\begin{array}{l}11.3 \\
10.2\end{array}$ & $\begin{array}{r}10.8 \\
9.7\end{array}$ & 11.0 & 11.11 & 106 & 106 & 9.3 & 9.3 & 9.0 & 9.3 & 73 & \\
\hline & $\overrightarrow{0}$ & & & 1.1 & 10.0 & 10.0 & 100 & 10.0 & 3.2 & 9.2 & & & 82 & S.6 \\
\hline 5 & 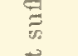 & & 8.4 & 8.0 & 10.2 & 10.2 & $\ldots$ & - - & $\begin{array}{l}9.0 \\
9.0\end{array}$ & 9.0 & 10.2 & 10.5 & 8.4 & 8.5 \\
\hline 6 & $\overleftrightarrow{\circ}$ & & 6.1 & 5.7 & 8.5 & 8.5 & - - & - - & 9.0 & 9.0 & 0.1 & 9.4 & 8.4 & 8.5 \\
\hline 7 & $\stackrel{\underbrace{\circ}_{0}}{\Xi}$ & & $\begin{array}{l}3.0 \\
2.3\end{array}$ & $\begin{array}{l}2.6 \\
1.9\end{array}$ & $\begin{array}{l}6.4 \\
5.1\end{array}$ & $\begin{array}{l}6.4 \\
5.1\end{array}$ & $\cdots$ & $\cdots$ & 7.5 & 7.5 & 7.4 & 7.8 & $\begin{array}{l}8.4 \\
8.4\end{array}$ & 8.8 \\
\hline$s$ & 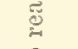 & & 2.0 & 1.7 & 3.2 & 3.2 & $\ldots$ & $\cdots$ & 5.5 & 5.5 & 6.4 & 6.8 . & 7.7 & 8.1 \\
\hline 9 & $\stackrel{\circ}{\square}$ & & $\begin{array}{l}2.0 \\
2.2\end{array}$ & $\begin{array}{l}1.7 \\
1.9\end{array}$ & $\begin{array}{l}3.2 \\
3.2\end{array}$ & $\begin{array}{l}3.2 \\
3.2\end{array}$ & 4.0 & 4.0 & 4.6 & 4.6 & 5.0 & 5.4 & 6.0 & 6.4 \\
\hline & $\stackrel{0}{\because}$ & & & & 3.3 & 3.3 & & & 4.4 & 4.4 & 19 & +8 & & \\
\hline 10 & 足 & & 3.5 & 3.2 & 3.4 & 3.4 & 5.2 & 5.2 & $\begin{array}{l}4.4 \\
4.4\end{array}$ & $\begin{array}{l}4.1 \\
4.4\end{array}$ & $\begin{array}{l}4.2 \\
4.1\end{array}$ & $\begin{array}{l}4.6 \\
4.5\end{array}$ & 4.4 & 4.8 \\
\hline 11 & $\overline{8}$ & & 4.8 & 4.5 & 4.2 & 4.2 & 5.5 & 5.5 & 4.4 & 4.4 & 4.1 & 4.5 & 3.4 & 3.5 \\
\hline Foon & 8.3 & 7.6 & 70 & $6 . ?$ & G.1 & (6.) 1 & 0.2 & 6.2 & $\begin{array}{r}4.4 \\
4.4\end{array}$ & $\begin{array}{l}4.4 \\
4.4\end{array}$ & $\begin{array}{l}4.1 \\
4.1\end{array}$ & $\begin{array}{l}4.5 \\
4.5\end{array}$ & $\begin{array}{l}3.4 \\
3.4\end{array}$ & $\begin{array}{l}3.5 \\
3.5\end{array}$ \\
\hline & 9.1 & 8.4 & 1.0 & 0.8 & 0.1 & 0.1 & 0.2 & 0.2 & 5.0 & 5.11 & 4.3 & 4.7 & 3.2 & 3.6 \\
\hline 1 & 9.2 & 8.5 & 9.2 & 8.9 & 7.0 & 7.0 & 7.5 & 7.5 & 5.7 & 5.7 & 4.8 & 5.2 & 3.2 & $3.6^{3}$ \\
\hline & 9.3 & $\therefore .6$ & & & & & 8.11 & 8.0 & & & & & 3.2 & 3.6 \\
\hline 2 & 9.3 & 8.6 & 10.2 & 9.9 & $\$ .3$ & 8.3 & 9.0 & 0.0 & 7.0 & 7.0 & 5.9 & 6.3 & 3.2 & 3.6 \\
\hline & 9.3 & 8.6 & & & 8.5 & 8.5 & 9.0 & 9.0 & & & & & 4.0 & 4.4 \\
\hline 3 & 9.2 & 8.5 & 11.3 & 10.0 & 0.2 & 9.2 & 9.11 & 9.0 & 8.7 & 8.7 & 6.4 & 6.8 & 4.5 & 4.9 \\
\hline & & & 11.4 & 11.2 & 9.11 & 9.0 & 9.0 & 9.0 & 9.5 & 9.5 & & & & \\
\hline 4 & 7.4 & 6.8 & 11.5 & 11.3 & 8.3 & 8.3 & $9.1)$ & 0.0 & 10.4 & 10.5 & 6.8 & 7.2 & 6.2 & 0.6 \\
\hline & & & 11.0 & 10.8 & & & & & 10.4 & 10.5 & & & & \\
\hline 5 & 5.5 & 4.9 & $10.1\}$ & 9.5 & 7.2 & 7.2 & 9.1 & 9.1 & 10.4 & 10.5 & 7.0 & 7.4 & 7.4 & 7.8 \\
\hline 6 & 3.7 & 3.1 & 7.8 & 7.6 & 0.0 & 6.0 & 8.0 & 8.0 & 10.42 & $\begin{array}{l}10.3 \\
10.3\end{array}$ & 9.1 & 9.5 & 8.2 & 8.6 \\
\hline 7 & 2.9 & 2.3 & 4.2 & 4.1 & 5.1 & 5.1 & 6.6 & 6.6 & 9.0 & 9.1 & $\begin{array}{l}9.0 \\
1.0\end{array}$ & $\begin{array}{l}9.4 \\
0.4\end{array}$ & 8.8 & 9.2 \\
\hline 8 & 2.7 & 2.1 & & 4.1 & 4.3 & 4.3 & & 0.0 & & & & & 9.1 & 9.4 \\
\hline 8 & 2.6 & 2.0 & 3.4 & 3.3 & 4.1 & 4.1 & 5.4 & 5.4 & 7.4 & 7.5 & 9.0 & 9.4 & 9.0 & 9.4 \\
\hline & 9.11 & 2.4 & & & 4.1 & 4.1 & 4.8 & 4.5 & & & & & 9.11 & 1.4 \\
\hline 9 & 3.2 2 & 2.7 & 3.3 & 3.2 & 4.1 & 4.1 & 4.9 & 4.8 & 6.2 & 6.3 & 7.4 & 7.8 & 9.0 & 9.4 \\
\hline & & & 4.0 & 3.9 & 5.11 & 5.0 & 4.5 & 4.5 & 6.0 & 6.1 & & & 9.11 & 9. 4 \\
\hline 10 & 5.4 & 4.9 & 4.0 & 3.9 & 5.2 & 5.2 & 4.8 & 4.8 & 6.0 & 6.1 & 6.2 & 6.6 & 5.2 & 8.6 \\
\hline & & & & & & & 4. & 4.5 & (i.0 & 6.1 & & & & \\
\hline 11 & 6.4 & 5.9 & 6.0 & 5.9 & 6.4 & 6.4 & $\begin{array}{l}4.5 \\
5.1\end{array}$ & $\begin{array}{l}4.8 \\
5.1\end{array}$ & 6.2 & 10.4 & 5.8 & 6.2 & 7.3 & 7.7 \\
\hline Midn't & 9.0 & 8.5 & 8.0 & 7.9 & $\ldots$ & $\ldots$ & 5.6 & 5.6 & 6.5 & 6.7 & 5.6 & 6.0 & 6.0 & 6.4 \\
\hline
\end{tabular}

Ibetwen the 20th am 27th of July the olservations do not appear sufficiently regular to promise any reliable resultis.

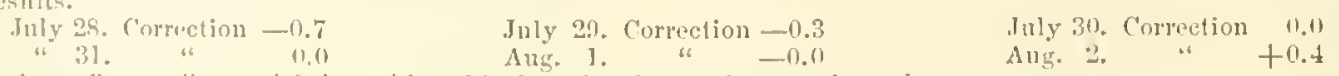

Anc. 3. " $\quad+0.4$ After this date the olsservations are irregular.

(I) the : the tho yours slipped of the whel.

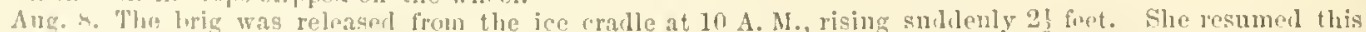
position upou very slight disturluance of the extorual ice, and is now on an even keel for thr. first time in eleven

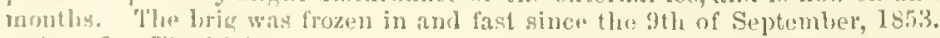

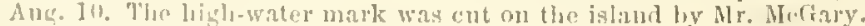

Ang. 1l. Tlh: wathing of the ships was commencerl. Thilal olsurvations wore resumed on the bath. Tho rongistur is k"pt in fithoms and fect. 
Setries IV._-'L'1daf, Onservations fron Seipember 7 to Octonel 22, 1854.

Hourly observations on the pulley-gange. Adopted reading of mean level 7.0, expressed in units of the scale. Increasing numbers indicate rise of water.

\section{September, 1854.}

\begin{tabular}{|c|c|c|c|c|c|c|c|c|c|c|c|c|c|c|c|c|c|c|c|c|}
\hline $\begin{array}{l}\text { Mean } \\
\text { solar } \\
\text { hour. }\end{array}$ & 7th. & Sth. & $9 \mathrm{th}^{\mathrm{H}} \mathrm{.}$ & 10th. & $11 \mathrm{th}$. & $120 \mathrm{~h}$. & 13th. & 14 th. & 16 th. & 17 th. & 1 sth. & 19th. & 20 th. & 21 st. & $22 d$. & 231. & 24 th. & $25 \mathrm{th}$. & $261 \mathrm{~h}$. & $2 i$ th. \\
\hline 1 & $\cdots$ & 10.0 & 13.5 & 13.0 & 10.0 & 10.0 & 8.0 & 6.0 & $\ldots$ & 5.0 & 7.0 & $\bigcap .0$ & 9.0 & 10.0 & 11.2 & ] 2.0 & 11.0 & 13.0 & 13.0 & 10.0 \\
\hline 2 & $\cdots$ & 5.0 & 13.5 & 11.11 & 11.0 & 14.11 & 9.0 & 7.0 & $\ldots$ & 5.0 & 6.0 & 8.11 & 7.5 & 8.0 & 9.0 & 10.01 & 10.0 & 14.0 & 11.11 & 12.0 \\
\hline 3 & $\ldots$ & 2.0 & 11.11 & 7.11 & 11.0 & 11.0 & 10.01 & 4.0 & - . & 6.11 & 0.0 & 7.0 & 5.0 & 6.0 & 4.0 & 7.11 & $\therefore 0$ & 10,11 & 10.0 & 10.0 \\
\hline 4 & $\ldots$ & -1.0 & 7.11 & 4.7 & 9.0 & 9.0 & 4.0 & 3.0 & $\ldots$ & 7.0 & 5.0 & 5.0 & 3.11 & 5.11 & 3.0 & 3.0 & 1.0 & 7.0 & 8.11 & $\therefore .0$ \\
\hline 5 & - - & -1.7 & 5.11 & 3.11 & 7.0 & 6.11 & 6.11 & 4.0 & - - - & 8.0 & 6.0 & 5.0 & 2.0 & 4.0 & 2.0 & 0.11 & 3.5 & 4.11 & if. (1 & 4.0 \\
\hline 6 & $\cdots$ & -1.7 & 3.11 & 1. (1) & 5.0 & 4.11 & 4.11 & 5.0 & - - - & 9.0 & 7.0 & 7.0 & 4.0 & 5.0 & 3.0 & -11.7 & 2.0 & 3.11 & 3.0 & $2.1)$ \\
\hline 7 & $\cdots$ & -1.0 & 2.2 & -1.0 & 3.1 & 2.0 & 0.11 & 10.0 & -- & 9.11 & 9.0 & 8.11 & 6.11 & (1.01 & 5.0 & 2.11 & 2.0 & 2.0 & 11.11 & 0.0 \\
\hline 8 & $\ldots$ & 0.0 & 1.0 & -1.11 & (1.,0) & 0.0 & 1.11 & $\therefore .11$ & $\ldots$ & 9.0 & 4.5 & 10.0 & 7.0 & 7.0 & (i. (1) & 4.11 & 2.0 & 0.0 & 1.0 & -1.0 \\
\hline 9 & $\cdots$ & 2.0 & 4.11 & 11.0 & 0.19 & 1.11 & 2.0 & 9.0 & $\cdots$ & 8.0 & 7.5 & 10.11 & 8.0 & 8.5 & 8.0 & 7.0 & 5.11 & 1.11 & $3.11^{\circ}$ & 1.0 \\
\hline 10 & - . & 9.0 & 5.0 & 4.0 & 2.0 & 3.0 & 3.11 & 10.0 & $\cdots$ & 0.0 & 9.0 & 9.5 & 9.11 & 10.0 & 10.0 & 10.01 & 10.0 & 3.11 & 4.0 & 3.0 \\
\hline 11 & $\cdots$ & 14.0 & 6.5 & --- & 4.0 & 4.0 & 5.0 & 4.0 & 4.0 & 5.0 & 7.0 & 8.5 & 9.5 & 11.5 & 12.5 & 11.0 & 12.11 & li.11 & 7.11 & 6.0 \\
\hline Noon & -- & 12.0 & 11.0 & $\cdots$ & 7.0 & 7.0 & 6.11 & & 4.11 & 5.11 & 6.5 & 8.11 & -- & 10.0 & 13.0 & 13.11 & 13.11 & 12.5 & 10.11 & 9.0 \\
\hline 1 & ... & 11.0 & $\begin{array}{l}13.0 \\
14.0\end{array}$ & 9.11 & 9.11 & 3.11 & $\therefore .0$ & & 5.0 & 5.0 & 6.0 & 7.5 & $\cdots$ & 8.0 & 10.0 & 11.0 & 12.0 & 13.0 & 12.0 & 10.0 \\
\hline 2 & $\cdots$ & 10.0 & 10.0 & 11.0 & 10.0 & 4.0 & 9.0 & $\Xi$ & 7.0 & 6.0 & 5.5 & 7.0 & $\cdots$ & 6.0 & 8.0 & 10.0 & 10.0 & 12.0 & 13.0 & 12.0 \\
\hline 3 & - - & 8.0 & 7.0 & 8.0 & 8.11 & 9.0 & 9.0 & 8 & 8.0 & 7.0 & 5.0 & 6.7 & 4.0 & 0.0 & 0.0 & 7.11 & 9.0 & 11.0 & 11.0 & 13.0 \\
\hline 4 & $\cdots$ & (j,0 & 4.0 & 6.0 & 9.0 & 10.0 & 9.0 & $\Xi$ & 9.0 & 8.0 & 7.0 & 6.5 & 2.0 & 1.0 & $3.1)$ & 5.0 & 19.01 & 7.0 & 9.0 & 10.0 \\
\hline 5 & $\ldots$ & 4.0 & 2.0 & $\cdots$ & 10.0 & 8.0 & 9.0 & $\cong$ & S.0 & 9.0 & 8.0 & 6.0 & 3.0 & 1.5 & 1.0 & 1.0 & 3.0 & 6.0 & 6.0 & 9.0 \\
\hline 6 & $\cdots$ & 2.0 & 0.0 & $\cdots$ & 10.5 & 4.0 & 6.0 &. & 7.0 & 10.0 & 9.0 & 5.0 & 4.0 & 3.0 & 2.0 & 0.0 & 2.0 & 4.0 & 4.0 & 7.0 \\
\hline 7 & $\cdots$ & 1.0 & -0.5 & $\cdots$ & 9.11 & 1.0 & 4.11 & $\stackrel{+}{\leftrightarrows}$ & 8.0 & 10.0 & 10.0 & 8.0 & 6.0 & 4.0 & 3.11 & 0.11 & 1.0 & 2.0 & 3.0 & 3.0 \\
\hline 8 & $-\cdots$ & 0.11 & -0.5 & $\cdots$ & 5.0 & 0.0 & 3.0 & 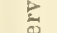 & 9.0 & 10.4 & 10.5 & 11.0 & 8.0 & 7.0 & 5.0 & 4.0 & 2.0 & 0.0 & 0.0 & 0.0 \\
\hline$?$ & 11.0 & 5.0 & 4.9 & 2.11 & 1.5 & 1.5 & 4.0 & in & 10.0 & 10.0 & 10.5 & 11.5 & 10.0 & 9.5 & 7.5 & $\cdots$ & 5.0 & 5.0 & 1.0 & 1.0 \\
\hline 10 & 13.0 & 8.0 & 6.0 & 1.0 & 3.0 & 3.0 & 5.0 & 5 & 9.0 & 10.0 & 12.0 & 12.0 & 12.0 & 12.0 & 11.5 & $\cdots$ & 8.11 & 8.0 & 4.0 & 4.0 \\
\hline 11 & 14.5 & 11.0 & 101,0 & 7.0 & 7.0 & 5.6 & 54 & & 8.0 & 10.4 & 11.0 & 12.6 & 13.0 & 14.0 & 111.11 & -- & 9.0 & 11.0 & 7.0 & i. 0 \\
\hline Mirln't & 14.2 & 13.0 & 12.0 & 10.0 & 8.6 & 7.0 & 7.11 & & 6.6 & 9.0 & 10.0 & 11.6 & 11.0 & 13.0 & 9.0 & $\cdots$ & 10.0 & 12.0 & 9.5 & 8.0 \\
\hline
\end{tabular}

\begin{tabular}{|c|c|c|c|c|c|c|c|c|c|c|c|c|c|c|c|c|c|c|c|}
\hline \multicolumn{4}{|c|}{ Sept. 1854.} & \multicolumn{11}{|c|}{ October, 1854.} & \multirow[b]{2}{*}{ 1sth. } & \multirow[b]{2}{*}{$19 \mathrm{th}}$. & \multirow[b]{2}{*}{20 th. } & \multirow[b]{2}{*}{ 21st. } & \multirow[b]{2}{*}{$22 d}$. \\
\hline $\begin{array}{l}\text { Mean } \\
\text { solar } \\
\text { hour. }\end{array}$ & esth. & 29 th. 3 & $30 \mathrm{th}$. & Ist. & thb. & 5th. & fith. 7 th. & Sth. & 9th. 1 & 10 th. & 11th. & 12 th. & 15 th. & 17th. & & & & & \\
\hline 1 & 9.0 & 8.0 & --- & 9.0 & $-\cdots$ & 6.0 & 10.1112 .11 & 14.0 & 8.01 & $13.0^{\prime}$ & 10.0 & 8.5 & (i.0) & $1 \ldots$ & 5.0 & $\ldots$ & 6.0 & 5.0 & $\ldots$ \\
\hline 2 & 12.0 & $10.1 ?$ & $\ldots$ & 8.0 & $\ldots$ & 4.0 & 7.1110 .0 & 12.2 & 7.01 & 12.0 & - . & 14.5 & 7.0 & - - - & 4.11 & ... & 4.0 & & $\ldots$ \\
\hline 3 & 13.0 & 9.0 & 8.0 & 0.0 & $\ldots$ & (1.0 & $5.0 \quad 7.11$ & 7.0 & 4.111 & 10.0 & 9.0 & 10.0 & 5.0 & $3 .(1$ & 3.0 & 1.0 & 3.0 & & 1.0 \\
\hline 4 & 10.0 & 8.0 & 9.0 & & $\ldots$ & -1.2 & $3.0 \quad 1.0$ & 5.0 & 2.0 & 7.0 & 8.0 & 7.11 & 10.0 & 4.0 & 4.0 & 0.0 & 2.0 & 0. & 0.0 \\
\hline 5 & 9.0 & 8.0 & 8.0 & & --- & -1.0 & $-1.0 \quad 2.0$ & 2.0 & 1.0 & 4.0 & 4.0 & 5.0 & 9.0 & 5.0 & 6.0 & 2.11 & 1.11 & 1 & 0.5 \\
\hline 6 & 5.5 & 7.0 & 7.0 & & $\ldots$ & 0.0 & $0.4 \quad 1.11$ & 1.0 & 0.0 & 0.0 & 2.0 & 4.0 & 40 & 6.0 & (i.4 & 3.0 & 3.0 & & 1.5 \\
\hline 7 & 3.0 & 6.0 & 0.0 & & 4.11 & 4.11 & $4.0,1.11$ & 0.0 & 11.11 & 1.0 & 1,0 & 3.11 & 7.0 & 7.0 & 7.0 & 6.0 & 5.0 & & 1.0 \\
\hline 8 & 1.0 & 4.0 & 5.10 & $\dot{\Xi}$ & 5.11 & 7.0 & 7.05 .0 & -1.0 & 0.5 & 1.5 & 0.0 & 2.0 & li. 0 & 8.0 & 8.0 & 9.11 & 7.0 & & 3.0 \\
\hline 9 & 2.0 & 3.0 & 5.0 & ٍ & 10.0 & 10.0 & $1(1.0 \quad \therefore .5$ & 7.0 & 7.11 & 2.5 & 2.0 & 3.5 & 5.0 & 9.0 & 11.0 & 12.0 & 111.11 & & 7.0 \\
\hline 10 & 3.11 & 2.0 & 4.11 & 80 & 10.0 & 11.0 & 11.511 .5 & 9.01 & 10.0 & 5.0 & 5.5 & 5.0 & 4.0 & 10.0 & 10.11 & 11.5 & 11.0 & 11.0 & 10.0 \\
\hline 11 & 7.0 & 4.0 & 3.0 &. & 9.0 & 12.0 & 12.013 .0 & 11.21 & 10.0 & 7.0 & 11.0 & 6.0 & 3.0 & 8.0 & 8.0 & 10.0 & 12.0 & 12. & 13.0 \\
\hline Noon & 9.0 & (3.0 & 4.01 & m & 8.0 & 13.0 & 12.014 .0 & 13.01 & 12.01 & 10.0 & 13.11 & 8.0 & 4.0 & 6.0 & 7.0 & 8.0 & 10.0 & 12 & 13.0 \\
\hline 1 & 12.0 & 7.0 & 6.0 & $\stackrel{E}{E}$ & ti. 0 : & 7.0 & 12.013 .0 & 13.01 & 13.01 & 13.0 & 14.0 & 111.0 & 5.2 & 5.11 & 6.0 & 7.5 & 8.0 & 10 & 12.0 \\
\hline 2 & 10.0 & 9.0 & 7.0 & ت्己 & 4.0 & 4.0 & $8.5 \quad 9.0$ & 12.01 & 14.01 & 14.0 & 13.0 & 13.0 & 5.0 & 4.0 & 5.0 & 4.11 & 3.0 & & 0.11 \\
\hline 3 & 11.0 & 10.0 & 8.0 & $\Xi$ & 2.0 & 2.11 & $7.11 \quad 5.0$. & 8.0 & 9.01 & 12.5 & 12.0 & 14.0 & 6.0 & 3.0 & 2.0 & 1.0 & 0.5 & & 4.11 \\
\hline 4 & 12.0 & 11.5 & 9.0 & 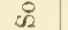 & 0.0 & 0.0 & $3.0 \quad 3.0$ & 3.11 & 7.11 & 9.0 & 10.0 & 14.0 & 7.0 & 4.0 & 2.0 & 1.0 & 0.0 & 2. & 1.5 \\
\hline 5 & 10.0 & 11.0 & 16.0 & & 0.0 & -2.0 & $1.0(1.1)$ & 2.0 & 5.0 & 8.11 & 7.0 & 11.0 & 8.0 & 5.0 & 3.0 & 2.0 & $1.0^{\prime}$ & 0.0 & 0.0 \\
\hline ii & 8.0 & 11.0 & 10.0 & & 5.0 & 3.0 & $011 \quad 0.0$ & 1.0 & 3.11 & 4.11 & 4.0 & 9.0 & 9.0 & 7.11 & 4.0 & 6.0 & 3.0 . & - 1.0 & 1.0 \\
\hline 7 & 6.11 & 9.0 & 9.0 & & 9.0 & 7.0 & - $11.5 \quad 2.0$ & 0.0 & 1.0 & 2.0 & 3.0 & 7.4 & 9.11 & 7.11 & 7.0 & 7.0 & 4.0 & 1. & 3.11 \\
\hline 8 & 4.11 & 6.0 & 8.11 & & 10,0 & 9.0 & $\begin{array}{ll}11.11 & 5.10\end{array}$ & 1.11 & 0.0 & 0.5 & 2.0 & 6.0 & 10.0 & $7.0 !$ & 9.5 & 8.0 & 7.0 & 3. & 7.11 \\
\hline 9 & 2.11 & 3.0 & 7.0 & & 12.11 & 13.11 & $9.11 \quad 7.1)$ & 4.11 & 3.0 & 3.19 & 3.0 & 4.0 & 8.0 & 8.0 & 12.11 & 9.0 & 8. & & 10.11 \\
\hline 10 & 3.11 & 4.0 & 5.5 & & 13.11 & 13.2 & $12 .(10.0$ & 6.0) & 5.5 & 4.0 & 4.11 & 6.11 & 6.0 & 11.0 & 16,0 & 10.0 & 9.1 & 10. & 12.0 \\
\hline 11 & 5.11 & 5.5 & 5.0 & & 14.11 & 14.0 & $11.013 .6^{\circ}$ & 9.11 & 8.0 & 7.0 & 7.0 & 8.0 & 5.0 & 8.11 & 7.0 & 10.0 & 9.1 & & 13.11 \\
\hline Mirln't & 6.0 & 5.5 & 4.5 & & 13.0 & 14.0 & $13 .(; 14.0$ & 10.01 & 11.01 & 11.01 & 10.0 & 10.0 & 7.0 & 7.11 & 6.0 & 11.0 & 11.0 & 11 & 11.0 \\
\hline \multicolumn{20}{|c|}{ 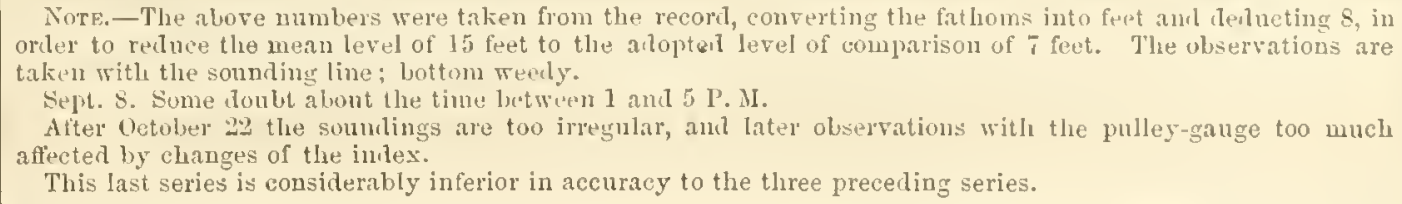 } \\
\hline
\end{tabular}




\section{Reduction of Tides, Van Rensselaer Harbor, 1853-'54.}

Having given the tidal record in a form ready for use, the observations next require to be properly tabnlated for the purpose of deducing empirically their laws, and for comparison with theory. In the United States Coast Survey two blank forms are in use for this tabulation; they have in their essential part been adopted as suitable for the Van Rensselaer Harbor tides, and werc used with permission of the Supcrintendent of the Survey. They are strictly applicable only for such cases where the dinnal inequality is comparatively small, or is at least not approximating to the production of single day tides. In order to show, at a glance, the gencral character of the tides mudcr discussion, they were plotted a second time, and are given in Plates I, II, and III; the observations having previously becn referred to the same mean level. From these diagrams it appears that the diurnal incquality is not of so great an effect as to render the use of the ordinary method of reduction mavailable; on the other hand, it is sufficiently large to require a special discussion for time and height. The extension of the scries of observations over a whole rear must be considered as a fortunate circumstance, since the results thereby gain considerably in accuracy over others deduced only from a few discomnected lunations.

'The tidal record would not be complete without the observations for direction and force of the wind, and for atmospheric pressure; the reader will find these records in my discussion of the metcorological matcrial of the expedition, in Vol. XI, Smithsonian Contributions to Knowledge, 1859.

'The following pages contain the first tabulation of the preceding record, viz: column 1 contains the date, civil reckoning, adopted for convenience sake. Column 2 gives the apparent time (civil reckoning) of the moon's superior and inferior transit over the Van Renssclaer meridian, obtained by adding nine minutes to the time of transit at Gircenwich, allowing for a difference of longitude of $4^{\mathrm{b}} 433^{\frac{1}{\mathrm{~m}}} \mathrm{~W}$. The nean time was converted into apparent time by applying the equation of time. 'The time for the lower transit was obtained by taking the mean of the time of the preceding and following upper transit. Columns 3 and 4 contain the apparent time of high and low water, taken from the record; in some cases a graphical method was resorted to, to obtain the instant of these pliases with greater precision. 'The equation of time lias been applied to the mean time in which the observations are cxpressed. Columns 5 and 6 contain the Imnitidal interval between the time of high water and low water, and the time of the transit of the moon immediately preceding, though in some cases, owing to the half-monthly inequality, it may be the second precerling, the establishment being abont $11^{3}$ hours. This transit of comparison has been called transit $F$ by Mr. Lubbock.' 'The next columns, 7 and 8 , give the height of high and Iow water, extracted from the preceding abstract. The remaining columns contain the moon's parallax and declination at noon.

\footnotetext{
2 Sec an Filementary I'reatise on the 'lides, by J. W. Lubboek, Esq., London, I 839.
} 
Table for tue Reduction of Tides.-No. 1.

Showing the times of IIjgh and Low Water, and the IIeiglits of High and Low Tides; together with the time of the Moon's passing the Meridian of the place, and the Lunitidal Intervals, at Tan Rensselaer Ilarbor during the months of October 10, 1853, to October 22, I855.

Series I.-From October 10 to Decenber 28, 1853.

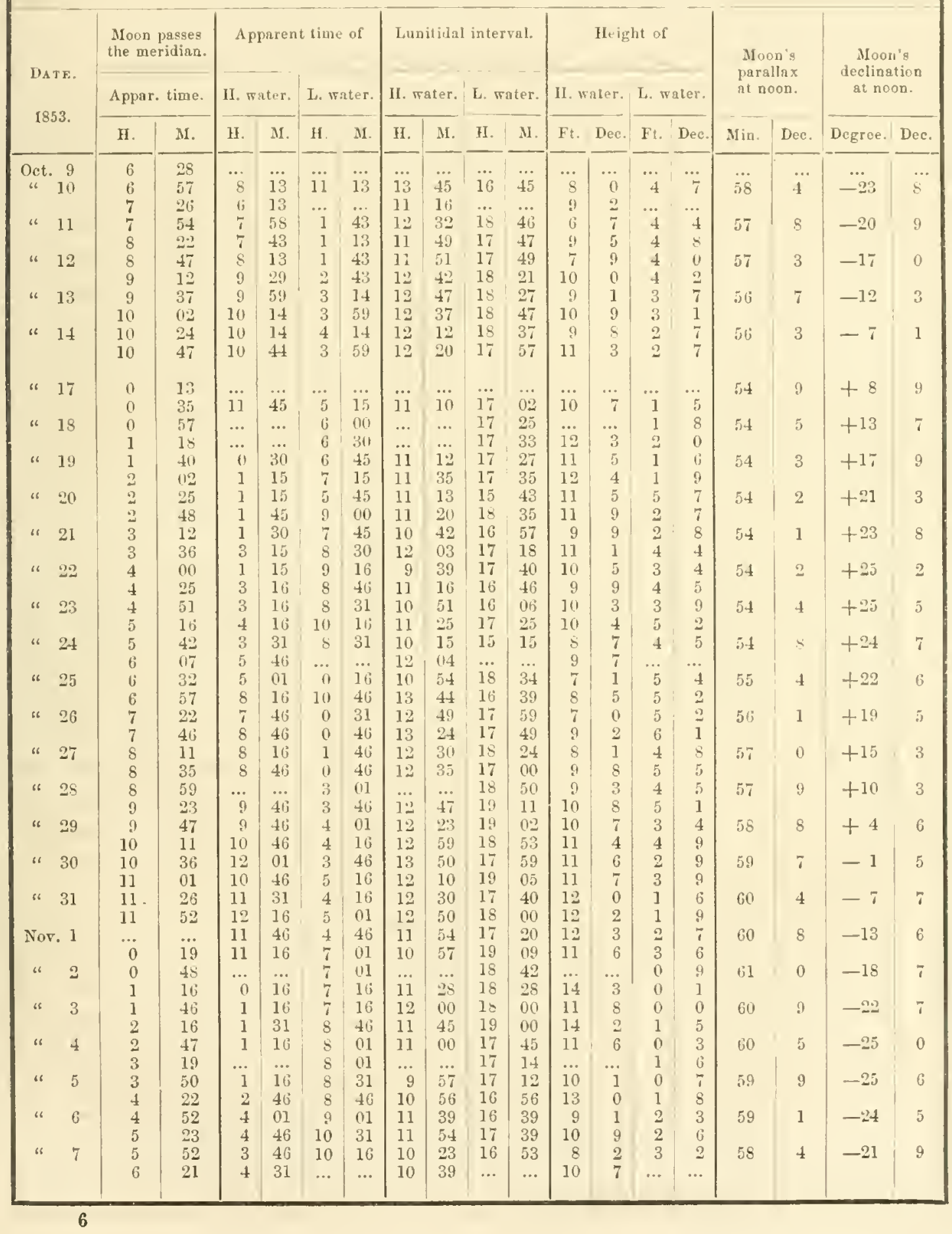




\begin{tabular}{|c|c|c|c|c|c|c|c|c|c|c|c|c|c|c|c|c|c|c|c|}
\hline \multirow{4}{*}{\multicolumn{2}{|c|}{$\begin{array}{l}\text { Date } \\
1853 .\end{array}$}} & \multicolumn{18}{|c|}{ Series 1. -From October 10 to Decenber $28,1853}$. \\
\hline & & \multirow{2}{*}{\multicolumn{2}{|c|}{$\begin{array}{l}\text { Moon passes } \\
\text { the meridian. } \\
\text { Appar. time. }\end{array}$}} & \multicolumn{4}{|c|}{ Apparent time of } & \multicolumn{4}{|c|}{ Lunitidul interval. } & \multicolumn{4}{|c|}{ Height of } & \multirow{2}{*}{\multicolumn{2}{|c|}{$\begin{array}{l}\text { Moon's } \\
\text { parallax } \\
\text { at noon. }\end{array}$}} & \multirow{2}{*}{\multicolumn{2}{|c|}{$\begin{array}{l}\text { Moon's } \\
\text { declioation } \\
\text { at noon. }\end{array}$}} \\
\hline & & & & \multicolumn{2}{|c|}{ II. water. } & L. w & ater. & II. W & ter. & L. $\#$ & ater. & L. W & ater. & II. w: & ater. & & & & \\
\hline & & II. & M. & II. & M. & II. & M. & II. & $\mathrm{M}$. & II. & M. & Ft. & Dec. & Ft. & Dec. & Min. & Dec. & Degree. & Dec. \\
\hline Nor & & 6 & 45 & 5 & 16 & 0 & 16 & 10 & 55 & 18 & 24 & 7 & 8 & 3 & 5 & 57 & 6 & -18 & 1 \\
\hline & & 5 & 14 & 6 & $46^{\circ}$ & 0 & 16 & 11 & 58 & 17 & 55 & 10 & 4 & 4 & 9 & & & & \\
\hline & 9 & 7 & 3s & 7 & 16 & 0 & 46 & 12 & 02 & 17 & 58 & 8 & 1 & 4 & 0 & 56 & 9 & -13 & 5 \\
\hline & 10 & $\begin{array}{l}5 \\
8\end{array}$ & $\begin{array}{l}122 \\
25\end{array}$ & $\begin{array}{l}7 \\
8\end{array}$ & $\begin{array}{l}31 \\
46\end{array}$ & $\begin{array}{l}1 \\
0\end{array}$ & $\begin{array}{l}16 \\
46\end{array}$ & $\begin{array}{l}11 \\
12\end{array}$ & $\begin{array}{l}53 \\
44\end{array}$ & $\begin{array}{l}18 \\
17\end{array}$ & $\begin{array}{l}02 \\
08\end{array}$ & $\begin{array}{r}11 \\
9\end{array}$ & $\begin{array}{l}0 \\
s\end{array}$ & $\begin{array}{l}4 \\
4\end{array}$ & $\begin{array}{l}4 \\
3\end{array}$ & & 3 & -8 & 4 \\
\hline & 10 & 8 & 48 & 9 & $\begin{array}{l}40 \\
46^{\circ}\end{array}$ & 2 & $\begin{array}{l}40 \\
31\end{array}$ & 13 & $\begin{array}{l}44 \\
21\end{array}$ & 18 & 29 & 10 & 7 & 5 & $\begin{array}{l}3 \\
5\end{array}$ & 56 & 3 & -8 & 4 \\
\hline & 11 & 9 & 09 & 10 & 01 & 2 & 46 & 13 & 13 & 18 & 21 & 9 & 5 & 2 & 3 & 55 & 7 & -3 & 1 \\
\hline & & $\begin{array}{l}9 \\
9\end{array}$ & 31 & 9 & 31 & 3 & 31 & 12 & 22 & 18 & 43 & 11 & 8 & 4 & 2 & & & & 3 \\
\hline & 12 & $\begin{array}{r}9 \\
10\end{array}$ & $\begin{array}{l}52 \\
13\end{array}$ & $\begin{array}{r}10 \\
9\end{array}$ & $\begin{array}{l}01 \\
46\end{array}$ & $\begin{array}{l}4 \\
3\end{array}$ & $\begin{array}{l}16 \\
31\end{array}$ & $\begin{array}{l}12 \\
11\end{array}$ & $\begin{array}{l}30 \\
54\end{array}$ & $\begin{array}{l}19 \\
15\end{array}$ & $\begin{array}{l}07 \\
100\end{array}$ & $\begin{array}{l}11 \\
11\end{array}$ & $\begin{array}{l}2 \\
5\end{array}$ & $\begin{array}{l}2 \\
3\end{array}$ & $\begin{array}{l}8 \\
8\end{array}$ & 55 & 2 & +2 & 3 \\
\hline " & 13 & 10 & 34 & 10 & 16 & 4 & 46 & 12 & 13 & 18 & 54 & 11 & 5 & 2 & 6 & 54 & 8 & +7 & 5 \\
\hline & & 10 & $\begin{array}{l}54 \\
15\end{array}$ & $\begin{array}{l}11 \\
10\end{array}$ & $\begin{array}{r}15 \\
45\end{array}$ & $\begin{array}{l}6 \\
5\end{array}$ & 15 & 12 & 41 & 20 & 02 & 11 & 0 & 2 & 5 & & & & \\
\hline & 14 & $\begin{array}{l}11 \\
11\end{array}$ & $\begin{array}{l}15 \\
37\end{array}$ & $\begin{array}{l}10 \\
10\end{array}$ & $\begin{array}{l}43 \\
45\end{array}$ & 5 & $\begin{array}{r}0 \\
30\end{array}$ & $\begin{array}{l}11 \\
11\end{array}$ & $\begin{array}{l}51 \\
30\end{array}$ & $\begin{array}{l}18 \\
18\end{array}$ & $\begin{array}{l}26 \\
36\end{array}$ & $\begin{array}{r}12 \\
9\end{array}$ & $\begin{array}{l}7 \\
8\end{array}$ & $\begin{array}{l}2 \\
2\end{array}$ & $\frac{1}{9}$ & 54 & 5 & +12 & 4 \\
\hline " & 15 & 11 & 59 & 11 & 30 & 5 & 30 & 11 & 53 & 18 & 15 & 13 & 3 & 0 & 7 & 54 & 2 & +16 & 8 \\
\hline & & $\ldots$ & $\ldots$ & 12 & 00 & 6 & 30 & 12 & 01 & 18 & 53 & 13 & 0 & 3 & 5 & & & & \\
\hline “" & 16 & $\begin{array}{l}0 \\
0\end{array}$ & $\begin{array}{l}21 \\
44\end{array}$ & 12 & $\begin{array}{l}30 \\
45\end{array}$ & 5 & 0 & 12 & 09 & 17 & 01 & 13 & 6 & 0 & 4 & 54 & 1 & +20 & 5 \\
\hline & 17 & 1 & 07 & $\begin{array}{l}11 \\
\ldots\end{array}$ & $\begin{array}{l}\text { to } \\
\ldots\end{array}$ & 7 & $\begin{array}{r}15 \\
0\end{array}$ & $\begin{array}{l}11 \\
\ldots\end{array}$ & $\begin{array}{l}01 \\
\ldots\end{array}$ & $\begin{array}{l}18 \\
18\end{array}$ & $\begin{array}{l}54 \\
16\end{array}$ & $\begin{array}{l}10 \\
\ldots\end{array}$ & $\begin{array}{l}9 \\
\ldots\end{array}$ & $\begin{array}{l}3 \\
1\end{array}$ & $\begin{array}{l}1 \\
3\end{array}$ & 54 & 0 & +23 & 2 \\
\hline & & 1 & 31 & 11 & 30 & $i$ & 45 & 111 & $\dddot{23}$ & 17 & 38 & $\dddot{13}$ & $\dddot{0}$ & 3 & 3 & 24 & 0 & +20 & 2 \\
\hline “ & 18 & 1 & 55 & 0 & 45 & 7 & 15 & 11 & 14 & 17 & 44 & 13 & $T$ & -0 & 1 & 54 & 0 & +25 & 0 \\
\hline & & 2 & 20 & 1 & 15 & 8 & 15 & 11 & 20 & 18 & 201 & 12 & 1 & 1 & 2 & & & & \\
\hline “ & 19 & $\frac{2}{3}$ & $\begin{array}{l}44 \\
09\end{array}$ & 1 & 14 & 7 & 14 & 10 & 54 & 16 & 54 & $?$ & $\varepsilon$ & 1 & 9 & 54 & 1 & +25 & 7 \\
\hline " & 20 & 3 & $\begin{array}{l}09 \\
34\end{array}$ & $\begin{array}{l}1 \\
1\end{array}$ & $\begin{array}{l}44 \\
44\end{array}$ & $\begin{array}{l}8 \\
8\end{array}$ & $\begin{array}{l}14 \\
14\end{array}$ & $\begin{array}{l}11 \\
10\end{array}$ & $\begin{array}{l}60 \\
35\end{array}$ & $\begin{array}{l}17 \\
17\end{array}$ & $\begin{array}{l}30 \\
05\end{array}$ & 13 & 0 & $\frac{2}{2}$ & $\begin{array}{l}3 \\
6\end{array}$ & 54 & 4 & +25 & 2 \\
\hline & & 3 & 59 & 2 & $\begin{array}{l}44 \\
14\end{array}$ & 8 & $\begin{array}{l}14 \\
59\end{array}$ & 10 & 40 & 17 & 25 & 11 & $\begin{array}{l}1 \\
5\end{array}$ & 3 & $\begin{array}{l}0 \\
6\end{array}$ & 24 & 4 & T-J & 2 \\
\hline " & 21 & 4 & 24 & 2 & 29 & 7 & 59 & 10 & 30 & 16 & 010 & 8 & 0 & 3 & 2 & 54 & 7 & +23 & 5 \\
\hline & & 4 & 49 & $\frac{2}{0}$ & 44 & 11 & 14 & 10 & 20 & 18 & 50 & 10 & 7 & 3 & 6 & & & & \\
\hline & 22. & $\begin{array}{l}5 \\
5\end{array}$ & $\begin{array}{l}14 \\
37\end{array}$ & $\frac{2}{3}$ & $\begin{array}{l}49 \\
43\end{array}$ & $\begin{array}{r}8 \\
11\end{array}$ & $\begin{array}{l}44 \\
13\end{array}$ & $\begin{array}{l}10 \\
10\end{array}$ & $\begin{array}{l}110 \\
29\end{array}$ & $\begin{array}{l}15 \\
17\end{array}$ & $\begin{array}{l}55 \\
59\end{array}$ & $\begin{array}{r}7 \\
10\end{array}$ & $\begin{array}{l}4 \\
1\end{array}$ & $\begin{array}{l}5 \\
3\end{array}$ & $\begin{array}{l}1 \\
7\end{array}$ & 55 & 3 & +20 & 7 \\
\hline " & 23 & 6 & 01 & 6 & 13 & 10 & 28 & 12 & 36 & 16 & 51 & 7 & 1 & 6 & 6 & 55 & 9 & +16 & 9 \\
\hline & & 6 & 25 & 4 & 43 & $\ldots$ & $\ldots$ & 10 & 42 & $\ldots$ & $\ldots$ & 9 & 9 & $\ldots$ & $\ldots$ & & & & \\
\hline & 24 & $\begin{array}{l}6 \\
7\end{array}$ & $\begin{array}{l}48 \\
11\end{array}$ & 8 & 13 & 0 & 43 & 13 & $4 \mathrm{~s}$ & 18 & 32 & 8 & 5 & 3 & 7 & 56 & 8 & +12 & 3 \\
\hline “ & 25 & 7 & 34 & 7 & 48 & 0 & $\begin{array}{l}13 \\
43\end{array}$ & $\begin{array}{l}11 \\
12\end{array}$ & $\begin{array}{l}15 \\
37\end{array}$ & 17 & $\begin{array}{l}45 \\
55\end{array}$ & $\begin{array}{l}? \\
8\end{array}$ & $\begin{array}{l}5 \\
8\end{array}$ & $\begin{array}{l}3 \\
3\end{array}$ & $\begin{array}{l}4 \\
7\end{array}$ & 57 & 7 & +7 & 0 \\
\hline & & 7 & 56 & 6 & 57 & 1 & 42 & 11 & 23 & 18 & 31 & 10 & 3 & 6 & 9 & & & & \\
\hline “ & $20^{\circ}$ & 8 & 20 & 8 & 42 & 1 & 12 & 12 & $4 t^{\circ}$ & 17 & 38 & 9 & 4 & 4 & 2 & 58 & 6 & +1 & 2 \\
\hline & & 8 & 44 & 8 & 27 & 2 & 42 & 12 & 07 & 18 & 46 & 9 & 9 & 4 & 3 & & & & \\
\hline & 26 & $\begin{array}{l}9 \\
9\end{array}$ & $\begin{array}{l}118 \\
33\end{array}$ & $\begin{array}{l}? \\
9\end{array}$ & $\begin{array}{l}42 \\
12\end{array}$ & $\begin{array}{l}3 \\
4\end{array}$ & $\begin{array}{l}42 \\
12\end{array}$ & $\begin{array}{l}12 \\
12\end{array}$ & $\begin{array}{l}58 \\
04\end{array}$ & $\begin{array}{l}19 \\
19\end{array}$ & $\begin{array}{l}22 \\
28\end{array}$ & $\begin{array}{l}10 \\
11\end{array}$ & $\begin{array}{l}9 \\
2\end{array}$ & $\frac{2}{3}$ & $\begin{array}{l}7 \\
4\end{array}$ & 59 & 6 & -4 & 9 \\
\hline “ & 28 & 9 & 58 & 10 & 12 & 4 & 12 & 12 & 39 & 19 & 04 & 12 & 0 & 2 & 2 & 60 & 5 & -10 & 9 \\
\hline & & 10 & 24 & 11 & 11 & 4 & 41 & 13 & 13 & 19 & 105 & 12 & 5 & 2 & 6 & & & & \\
\hline “ & 29 & 10 & 51 & 11 & 41 & 4 & 41 & 13 & 17 & 18 & 43 & 13 & 8 & 0 & 7 & 61 & 0 & -16 & 5 \\
\hline & & 11 & 20 & 10 & 56 & 4 & 11 & 12 & 05 & 17 & 47 & 10 & 7 & 1 & 2 & & & 91 & \\
\hline$"$ & 30 & 11 & 50 & 10 & $56^{\circ}$ & 4 & 41 & 11 & 36 & 17 & 50 & 13 & 7 & 0 & 1 & 61 & 4 & & 1 \\
\hline D)e. & . 1 & (1) & $\ddot{21}$ & $\dddot{0}$ & $\dddot{11}$ & $\begin{array}{l}5 \\
5\end{array}$ & $\begin{array}{l}26 \\
41\end{array}$ & $\dddot{12}$ & $\dddot{2}$ & $\begin{array}{l}18 \\
17\end{array}$ & $\begin{array}{l}06 \\
51\end{array}$ & $\dddot{10}$ & $\ddot{\varepsilon}$. & $\begin{array}{r}1 \\
-1\end{array}$ & $\dot{2}$ & 61 & 4 & -24 & 3 \\
\hline & & () & 53 & 0 & 11 & 6 & 26 & 11 & 50 & 18 & 05 & 12 & 8 & -0 & 3 & & & & \\
\hline " & 2 & 1 & 25 & 0 & 10 & 6 & 10 & 11 & 17 & 17 & 17 & 9 & 5 & -2 & 1 & 61 & 0 & -25 & 7 \\
\hline & & 1 & 58 & 1 & 10 & 7 & 10 & 11 & 45 & 17 & 45 & 13 & 6 & 11 & 0 & & & & \\
\hline$a$ & 3 & 2 & 30 & 1 & 10 & 7 & 10 & 11 & 12 & 17 & 12 & 9 & 4 & $\rightarrow 0$ & 4 & 60 & 4 & -25 & 2 \\
\hline & & 3 & 03 & 1 & 55 & 8 & 41) & 11 & 25 & 18 & 10 & 12 & 8 & -0 & 1 & & & & \\
\hline$"$ & 4 & 3 & 32 & 1 & 54 & 6 & 39 & 10 & 51 & 15 & 36 & 8 & 3 & 0 & 1 & 59 & 6 & -22 & 9 \\
\hline & & 4 & 113 & 2 & 119 & 9 & 09 & 10 & 37 & 17 & 37 & 13 & 7 & 1 & 6 & & & & \\
\hline$"$ & 5 & 4 & $: 31$ & 2 & 39 & 8 & 54 & 10 & $3 i$ & 16 & 51 & 9 & 1 & 1 & 3 & 58 & 7 & -19 & 4 \\
\hline & & 5 & 1111) & 3 & 3!) & 9 & $3 ! r$ & 11 & us & 17 & 04 & 11 & 1 & 1 & 0 & & & & \\
\hline$"$ & 6 & 5 & 26 & 3 & 09 & $s$ & 24 & 10 & 09 & 15 & 24 & 6 & 1 & 2 & 3 & 57 & 7 & -14 & 9 \\
\hline & & 5 & 52 & 4 & 24 & 11 & $3 !$ & 10 & 58 & 18 & 13 & 10 & 3 & 1 & 0 & & & & \\
\hline$"$ & 7 & 6 & $1-4$ & 6 & 34 & $\ldots$ & $\ldots$ & 12 & 46 & $\ldots$ & $\ldots$ & 6 & 8 & $\ldots$ & $\ldots$ & 56 & 9 & -9 & 8 \\
\hline & & ij & 3 & 7 & 53 & 0 & 114 & 13 & 39 & 18 & 16 & 10 & 6 & 3 & 4 & & & & \\
\hline & 8 & $\frac{1}{i}$ & (11) & $\begin{array}{l}{ }^{6} \\
\ldots\end{array}$ & $\begin{array}{l}23 \\
\ldots\end{array}$ & $\begin{array}{l}1 \\
0\end{array}$ & $\begin{array}{l}11,4 \\
33\end{array}$ & $\begin{array}{l}11 \\
\ldots\end{array}$ & $\begin{array}{l}45 \\
\ldots\end{array}$ & $\begin{array}{l}18 \\
18\end{array}$ & $\begin{array}{l}54 \\
60\end{array}$ & $\begin{array}{r}7 \\
\ldots\end{array}$ & $\begin{array}{c}6 \\
\ldots\end{array}$ & $\begin{array}{l}3 \\
2\end{array}$ & $\begin{array}{l}5 \\
9\end{array}$ & 50 & 1 & & 4 \\
\hline " & $y$ & 7 & 42 & $x$ & 37 & 1 & 117 & 13 & 15 & 15 & 07 & $\dddot{11}$ & $\dddot{2}$ & 3 & ii & 55 & 4 & +1 & 0 \\
\hline & & 8 & 14.4 & $x$ & 07 & 2 & 187 & 12 & 25 & 18 & 4 & 11 & 8 & 5 & 2 & & & & \\
\hline & 10) & 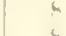 & 25 & ! & 37 & 2 & 07 & 13 & 33 & 18 & 25 & 11 & 1 & 5 & 3 & 54 & 9 & +6 & 3 \\
\hline & & g & 40 & 9 & 07 & 2 & 07 & 12 & 42 & 10 & 03 & 9 & 2 & 5 & 3 & & & & \\
\hline
\end{tabular}




\begin{tabular}{|c|c|c|c|c|c|c|c|c|c|c|c|c|c|c|c|c|c|c|c|}
\hline \multicolumn{20}{|c|}{ Series I.-From Octorer 10 to Dfcember $28,1853}$. \\
\hline \multirow{3}{*}{\multicolumn{2}{|c|}{$\begin{array}{l}\text { Date. } \\
1853 .\end{array}$}} & \multirow{2}{*}{\multicolumn{2}{|c|}{$\begin{array}{c}\text { Moon passes } \\
\text { the meridian. } \\
\text { Aprar. time. }\end{array}$}} & \multicolumn{4}{|c|}{ Apparent time of } & \multicolumn{4}{|c|}{ Lunitidal interval. } & \multicolumn{4}{|c|}{ Ileight of } & \multirow{2}{*}{\multicolumn{2}{|c|}{$\begin{array}{l}\text { Moon's } \\
\text { farallax } \\
\text { at noon. }\end{array}$}} & \multirow{2}{*}{\multicolumn{2}{|c|}{$\begin{array}{c}\text { Mons's } \\
\text { declination } \\
\text { at noon. }\end{array}$}} \\
\hline & & & & \multicolumn{2}{|c|}{ II. Water. } & L. W & ter. & 1I. $\pi$ & ater. & L. $\mathrm{w}$ & ater. & II. W & ater. & L. Wh & ater. & & & & \\
\hline & & II. & M. & II. & M. & II. & M. & 11. & M. & II. & M. & Ft. & Dec. & Ft. & Dec. & Min. & Dec. & Degree. & Dec. \\
\hline Dec. & 11 & 9 & 06 & 9 & 51 & 3 & 36 & 13 & 05 & $1 ?$ & 11 & 9 & 5 & 1 & 7 & 54 & 5 & +11 & 2 \\
\hline & & 9 & 27 & 9 & 36 & 3 & 36 & 12 & 30 & 18 & 50 & 7 & 8 & 1 & $S$ & & & & \\
\hline$"$ & 12 & 9 & 49 & 11 & 016 & 4 & 116 & 13. & $3: 1$ & 19 & 00 & 9 & 9 & 1) & 3 & 54 & 2 & +15 & 7 \\
\hline & & 10 & 10 & 11 & $00^{\circ}$ & 4 & 36 & $13^{\circ}$ & 17 & 19 & 09 & 9 & 6 & 2 & 8 & & & & \\
\hline 6 & 13 & 10 & 31 & 10 & 35 & 4 & 05 & 12 & 35 & 18 & $16^{\circ}$ & 11 & 0 & 3 & 5 & 54 & 0 & +19 & 6 \\
\hline & & 10 & 54 & 10 & 20 & 4 & 35 & 11 & 49 & 18 & 25 & 8 & 6 & 2 & 8 & & & & \\
\hline$"$ & 14 & 11 & 17 & 11 & 05 & 4 & 35 & 12 & 11 & 18 & 04 & 12 & 5 & 2 & 3 & 53 & 9 & +22 & 6 \\
\hline & & 11 & 41 & 11 & 115 & 6 & 05 & 11 & 48 & 19 & 11 & $s$ & 9 & 2 & 4 & & & & \\
\hline$"$ & 15 & $\ddot{0}$ & 06 & $\ldots$ & $\ldots$ & 5 & 05 & ... & $\ldots$ & 17 & 48 & ... & $\cdots$ & 2 & 4 & 53 & 9 & +24 & 7 \\
\hline " & 16 & $\begin{array}{l}0 \\
0\end{array}$ & $\begin{array}{l}06 \\
30\end{array}$ & $\begin{array}{l}1 \\
0\end{array}$ & $\begin{array}{l}04 \\
04\end{array}$ & $\begin{array}{l}6 \\
5\end{array}$ & $\begin{array}{l}04 \\
(1) 4\end{array}$ & $\begin{array}{l}13 \\
11\end{array}$ & $\begin{array}{l}23 \\
58\end{array}$ & $\begin{array}{l}18 \\
16\end{array}$ & $\begin{array}{l}23 \\
58\end{array}$ & $\begin{array}{l}13 \\
12\end{array}$ & $\begin{array}{l}2 \\
5\end{array}$ & $\begin{array}{l}5 \\
3\end{array}$ & $\begin{array}{l}5 \\
4\end{array}$ & 54 & 0 & +25 & 6 \\
\hline & & 0 & 55 & 0 & 19 & 7 & 19 & 11 & 49 & 18 & 49 & 12 & 8 & 1 & 6 & & & & \\
\hline$" 6$ & 17 & 1 & 20 & 0 & 34 & 6 & 03 & 11 & 39 & 17 & 08 & 7 & 5 & 2 & 7 & 54 & 2 & +25 & 4 \\
\hline & & 1 & 44 & $\ldots$ & ... & 5 & 15 & $\ldots$ & ... & 15 & 58 & $\ldots$ & ... & 0 & 6 & & & & \\
\hline$" 6$ & 18 & 2 & 19 & 1 & 03 & 7 & 03 & 11 & 19 & 17 & 19 & ... & $\cdots$ & $\cdots$ & $\cdots$ & 54 & 5 & +24 & 0 \\
\hline " & 19 & 2 & 34 & $\cdots$ & ... & $\cdots$ & 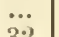 & $\ddot{i i}$ & $\dddot{0}$ & & $\ddot{0}$ & $\cdots$ & $\ldots$ & $\cdots$ & $\cdots$ & & & & \\
\hline & 19) & $\begin{array}{l}2 \\
3\end{array}$ & $\begin{array}{l}58 \\
22\end{array}$ & $\begin{array}{l}2 \\
1\end{array}$ & $\begin{array}{l}03 \\
32\end{array}$ & $\begin{array}{l}7 \\
8\end{array}$ & $\begin{array}{l}32 \\
112\end{array}$ & $\begin{array}{l}11 \\
10\end{array}$ & $\begin{array}{l}29 \\
34\end{array}$ & $\begin{array}{l}17 \\
17\end{array}$ & $\begin{array}{l}58 \\
04\end{array}$ & $\begin{array}{l}\ldots \\
\ldots\end{array}$ & $\begin{array}{l}\ldots \\
\ldots\end{array}$ & $\ldots$ & $\ldots$ & 54 & 8 & +21 & 5 \\
\hline$"$ & 20 & 3 & $46^{\circ}$ & 2 & 17 & 8 & 32 & 10 & 55 & 17 & 10 & $\dddot{10}$ & $\dddot{9}$ & $\ddot{1}$ & 4 & 55 & 3 & $+1 S$ & 0 \\
\hline & & 4 & 09 & 2 & 47 & 6 & 02 & 11 & 01 & 14 & 16 & 10 & 4 & $\ldots$ & $\ldots$ & & & & \\
\hline$"$ & 21 & $\overrightarrow{4}$ & 32 & 3 & 02 & 8 & 31 & 10 & 53 & 16 & 22 & 11 & 6 & 4 & 5 & 55 & 9 & +13 & 7 \\
\hline & & 4 & 54 & 3 & 46 & 9 & 31 & 11 & 14 & 16 & 59 & 11 & 5 & 4 & 4 & & & & \\
\hline$"$ & 22 & 5 & 17 & 4 & 31 & 9 & 16 & 11 & 37 & 16 & 22 & 9 & 1 & 5 & 1 & 56 & 5 & $+s$ & 7 \\
\hline & & 5 & 39 & 4 & 31 & 11 & 111 & 11 & 14 & 17 & 44 & 11 & 6 & 4 & 2 & & & & \\
\hline$"$ & 23 & 6 & (11 & 5 & 31 & 11 & 30 & 11 & 52 & 17 & 51 & 7 & 5 & 3 & 1 & 57 & 4 & +3 & 2 \\
\hline & & 6 & 24 & 4 & 30 & 11 & 30 & 10 & 29 & 17 & 29 & 8 & 8 & 2 & 9 & & & & \\
\hline " & 24 & 6 & 47 & 7 & 00 & $\ldots$ & 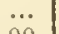 & 12 & 36 & $\ddot{10}$ & 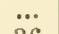 & 7 & 8 & ... & $\cdots$ & 58 & 3 & -2 & 6 \\
\hline$u$ & 25 & 7 & $\begin{array}{l}10 \\
34\end{array}$ & $\begin{array}{l}6 \\
5\end{array}$ & $\begin{array}{l}30 \\
00\end{array}$ & $\begin{array}{l}0 \\
1\end{array}$ & 00 & $\begin{array}{l}11 \\
12\end{array}$ & $\begin{array}{l}43 \\
50\end{array}$ & 17 & $\begin{array}{l}36 \\
28\end{array}$ & 9 & $\begin{array}{l}3 \\
0\end{array}$ & $\begin{array}{l}4 \\
?\end{array}$ & $\begin{array}{l}6 \\
4\end{array}$ & & & & \\
\hline & & 7 & $\begin{array}{l}04 \\
59\end{array}$ & 7 & 30 & 0 & $\begin{array}{l}1 . \\
15\end{array}$ & 11 & 56 & 17 & 05 & $\begin{array}{l}8 \\
7\end{array}$ & 3 & 4 & $\begin{array}{l}4 \\
1\end{array}$ & 59 & 2 & $-s$ & 5 \\
\hline$" i$ & 26 & 8 & 23 & 8 & 29 & 1 & 44 & 12 & 30 & 18 & 10 & 11 & 5 & 3 & 2 & 60 & 0 & -14 & 1 \\
\hline & & 8 & 50 & 8 & 14 & 2 & 44 & 11 & 51 & 18 & 45 & 9 & 2 & 4 & 8 & & & & \\
\hline$"$ & 27 & 9 & 18 & 9 & 28 & 1 & 59 & 12 & 38 & 17 & 36 & 11 & 2 & 1 & $\varepsilon$ & 60 & 7 & -19 & 1 \\
\hline & & 9 & 46 & 9 & 28 & 3 & 55 & 12 & 10 & 19 & 08 & 9 & 1 & 2 & 3 & & & & \\
\hline$"$ & 28 & 10 & 16 & 10 & 13 & 3 & 28 & 12 & 27 & 18 & 10 & 11 & 6 & 1 & 9 & 61 & 2 & -22 & 9 \\
\hline & & 10 & $4 S$ & 10 & 58 & 4 & 58 & 12 & 42 & 19 & 12 & 10 & 6 & 4 & 9 & & & & \\
\hline & & & & & Tits & II & $-F_{B}$ & I & & $A R I$ & & 1 & PRTL & 7,1 & & & & & \\
\hline Jan. & 27 & 10 & 58 & $\cdots$ & $\cdots$ & $\cdots$ & $\cdots$ & $\cdots$ & $\ldots$ & $\cdots$ & $\ldots$ & ... & $\cdots$ & $\cdots$ & $\cdots$ & 60 & 7 & -25 & 0 \\
\hline "6 & & 11 & 30 & ... & $\ddot{i r}$ & $\cdots$ & $\ldots$ & i.. & 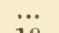 & i.. & 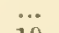 & ... & $\ldots$ & ... & ... & & & & \\
\hline 6 & 28 & $\ddot{0}$ & $\ddot{01}$ & 0 & $\begin{array}{l}17 \\
17\end{array}$ & 5 & 17 & 13 & 19 & 15 & 19 & 10 & 6 & 1 & 0 & 60 & 4 & -22 & 5 \\
\hline " & 29 & $\begin{array}{l}0 \\
0\end{array}$ & $\begin{array}{l}01 \\
31\end{array}$ & 0 & 17 & 6 & 17 & 12 & 47 & 18 & 47 & 12 & 4 & 2 & 6 & & & & \\
\hline & 29 & $\begin{array}{l}0 \\
1\end{array}$ & $\begin{array}{l}31 \\
01\end{array}$ & 0 & 17 & 6 & 17 & 12 & 16 & 18 & 16 & 11 & $?$ & 1 & 3 & 59 & 9 & $-1 S$ & 6 \\
\hline If & 30 & 1 & $\begin{array}{l}01 \\
27\end{array}$ & $\begin{array}{l}0 \\
0\end{array}$ & $\begin{array}{r}2 \\
16\end{array}$ & 8 & 02 & 11 & 31 & 19 & 31 & 13 & 3 & -1 & 8 & & & & \\
\hline & & 1 & $\begin{array}{l}27 \\
55\end{array}$ & $\begin{array}{l}0 \\
1\end{array}$ & $\begin{array}{l}16 \\
16\end{array}$ & 6 & 01 & 11 & 15 & 17 & 00 & $?$ & 0 & 0 & 5 & 59 & 1 & -13 & 7 \\
\hline$"$ & 31 & 2 & $\begin{array}{l}55 \\
20\end{array}$ & $\begin{array}{l}1 \\
0\end{array}$ & $\begin{array}{l}16 \\
46\end{array}$ & 7 & 46 & 11 & 49 & 18 & 19 & 13 & 7 & -0 & $\begin{array}{l}4 \\
3\end{array}$ & & & & \\
\hline & & 2 & 45 & 1 & 16 & 8 & 16 & 10 & 56 & 17 & 56 & 13 & 7 & 1 & 5 & 58 & 2 & -8 & 1 \\
\hline Feb. & 1 & 3 & $0 \mathrm{~s}$ & 2 & 46 & 8 & 46 & 12 & 01 & 18 & 01 & 11 & 1 & 1 & 3 & 57 & 3 & -2 & 4 \\
\hline & & 3 & 32 & 2 & 46 & 9 & 01 & 11 & 38 & 17 & 53 & 12 & 9 & 2 & 1 & & & & \\
\hline "f & 2 & 3 & 54 & 4 & 01 & 9 & 01 & 12 & 29 & 17 & 29 & 10 & 0 & 2 & 0 & 56 & 4 & +3 & 3 \\
\hline & & 4 & 16 & 2 & $46^{\circ}$ & 10 & 01 & 10 & 52 & 18 & 07 & 10 & 6 & 0 & 3 & & & & \\
\hline " & 3 & 4 & 38 & 4 & 01 & 8 & 46 & 11 & 45 & 16 & 30 & S & 5 & 3 & 4 & 55 & 6 & $+s$ & 7 \\
\hline & & 5 & 00 & 3 & 46 & ? & 31 & 11 & 08 & 16 & 53 & 8 & 6 & 2 & 2 & & & & \\
\hline$" \pi$ & 4 & 5 & 21 & 4 & 46 & 9 & 01 & 11 & 46 & 16 & 01 & 9 & 2 & 3 & 8 & 55 & 0 & +13 & 6 \\
\hline 6 & 5 & $\begin{array}{l}5 \\
6\end{array}$ & $\begin{array}{l}43 \\
06\end{array}$ & $\begin{array}{l}5 \\
6\end{array}$ & $\begin{array}{l}16 \\
16\end{array}$ & $\begin{array}{l}12 \\
12\end{array}$ & $\begin{array}{l}16 \\
16\end{array}$ & $\begin{array}{l}11 \\
12\end{array}$ & $\begin{array}{l}55 \\
33\end{array}$ & $\begin{array}{l}18 \\
18\end{array}$ & $\begin{array}{l}55 \\
33\end{array}$ & $\begin{array}{r}8 \\
10\end{array}$ & $\begin{array}{l}9 \\
5\end{array}$ & $\begin{array}{l}3 \\
5\end{array}$ & $\begin{array}{l}8 \\
0\end{array}$ & 54 & 5 & +17 & 8 \\
\hline & & 6 & 28 & 5 & 16 & 11 & $46^{\circ}$ & 11 & 10 & 17 & 40 & 10 & 7 & 4 & 5 & & & & \\
\hline$" t$ & 6 & 6 & 51 & 8 & 16 & 11 & 46 & 13 & 48 & 17 & 18 & 9 & 5 & 3 & $S$ & 54 & 3 & +21 & 3 \\
\hline & & 7 & 14 & 8 & 01 & 11 & 16 & 13 & 10 & 16 & 25 & 7 & 2 & 3 & 7 & & & & \\
\hline$"$ & 7 & 7 & 38 & 7 & 46 & $\ldots$ & $\ldots$ & 12 & 32 & $\ldots$ & $\ldots$ & 9 & 4 & ... & $\ldots$ & 54 & 1 & +23 & 9 \\
\hline & & 8 & 02 & 7 & 31 & 3 & 01 & 11 & 53 & 19 & 47 & 8 & 2 & 5 & 8 & & & & \\
\hline
\end{tabular}




\begin{tabular}{|c|c|c|c|c|c|c|c|c|c|c|c|c|c|c|c|c|c|c|c|}
\hline \multirow{4}{*}{\multicolumn{2}{|c|}{$\begin{array}{c}- \\
\text { DATE. } \\
1854 .\end{array}$}} & \multicolumn{18}{|c|}{ SERIES II.-From JaNUARY $2 S$ TU ApRIL $7,1854}$. \\
\hline & & \multirow{2}{*}{\multicolumn{2}{|c|}{$\begin{array}{l}\text { 3Ioon passes } \\
\text { the meridian. } \\
\text { Appar. time. }\end{array}$}} & \multicolumn{4}{|c|}{ Apparent time of } & \multicolumn{4}{|c|}{ Lunitidal interral. } & \multicolumn{4}{|c|}{ lleight of } & \multirow{2}{*}{\multicolumn{2}{|c|}{$\begin{array}{l}\text { Moon's } \\
\text { parallax } \\
\text { at noon. }\end{array}$}} & \multirow{2}{*}{\multicolumn{2}{|c|}{$\begin{array}{l}\text { Moon's } \\
\text { declination } \\
\text { at noon. }\end{array}$}} \\
\hline & & & & \multicolumn{2}{|c|}{ 1I. water. } & \multicolumn{2}{|c|}{ L. water. } & H. w & ater. & L. $\mathrm{w}$ & ater. & H. w: & ater. & L. $\mathrm{w}$ & ater. & & & & \\
\hline & & II. & II. & II. & M. & II. & ir. & $\mathrm{H}$. & II. & H. & M. & Ft. & Dec. & $\mathrm{Ft}$. & Dee. & Min. & Dee. & Degrea. & Dec. \\
\hline Feb. & 8 & $\varepsilon$ & 26 & $\begin{array}{r}10 \\
9\end{array}$ & 01 & $\frac{2}{3}$ & 46 & 13 & 59 & 19 & 09 & 9) & 5 & 4 & 7 & 54 & 1 & +25 & 4 \\
\hline$"$ & & 9 & $\begin{array}{l}50 \\
15\end{array}$ & 10 & $\begin{array}{l}15 \\
15\end{array}$ & $\begin{array}{l}3 \\
3\end{array}$ & $\begin{array}{l}15 \\
15\end{array}$ & 12 & $\begin{array}{ll}49 \\
25\end{array}$ & $\begin{array}{l}19 \\
18\end{array}$ & 3 & 6 & $\frac{7}{7}$ & $\begin{array}{l}3 \\
3 \\
3\end{array}$ & $\begin{array}{l}3 \\
8\end{array}$ & & 3 & & \\
\hline & & 9 & 41 & 9 & 45 & 4 & 45 & 12 & 30 & 19 & 55 & 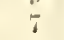 & 2 & 3 & 3 & 34 & 3 & & 7 \\
\hline “ & 10 & 10 & $0 i j$ & 10 & 15 & 6 & 15 & 12 & 34 & 11 & 00 & 9 & 2 & 3 & 7 & 54 & 6 & +24 & 9 \\
\hline & & 10 & 32 & 11 & 45 & 5 & 15 & 13 & 39 & 19 & & 6 & 6 & 1 & 5 & & & & \\
\hline “" & 11 & 10 & 57 & 11 & 45 & 5 & 00 & 13 & 13 & 18 & 54 & 10 & 5 & 1 & 3 & 54 & 9 & +22 & 8 \\
\hline " & 12 & $\begin{array}{l}11 \\
11\end{array}$ & $\frac{22}{46}$ & 11 & 45 & 4 & 45 & 12 & 48 & $1 \mathrm{~s}$ & 13 & 7 & 7 & 1 & 8 & & 1 & & \\
\hline & & $\ldots$ & 40 & 11 & $\begin{array}{l}4 . \\
15\end{array}$ & $\begin{array}{l}5 \\
6\end{array}$ & $\begin{array}{l}00 \\
30\end{array}$ & $\begin{array}{l}11 \\
11\end{array}$ & $\begin{array}{l}23 \\
29\end{array}$ & $\begin{array}{l}18 \\
19\end{array}$ & 3 & $\begin{array}{r}11 \\
8\end{array}$ & $\begin{array}{l}5 \\
4\end{array}$ & $\begin{array}{l}2 \\
2\end{array}$ & $\begin{array}{l}8 \\
3\end{array}$ & .3 & \pm & +13 & 8 \\
\hline “" & 13 & 0 & 10 & 11 & 15 & 5 & 45 & 11 & 115 & 17 & 59 & 12 & 6 & 0 & 7 & 55 & 8 & +15 & 6 \\
\hline & & 0 & 33 & 10 & 45 & 5 & 15 & 10 & 12 & & 05 & 10 & 6 & 4 & 1 & & & & \\
\hline :“ & 14 & 0 & 58 & $\ddot{0}$ & $\ldots$ & 6 & $\begin{array}{l}46 \\
16\end{array}$ & $\ldots$ & $\ldots$ & 18 & 13 & $\ldots$ & $\cdots$ & 2 & 4 & 56 & 3 & +10 & 7 \\
\hline “ & 15 & 1 & 44 & 0 & 31 & 6 & $\begin{array}{l}16 \\
46\end{array}$ & 11 & $\begin{array}{l}48 \\
10\end{array}$ & 8 & $\begin{array}{r}38 \\
25\end{array}$ & $\begin{array}{l}13 \\
11\end{array}$ & $\begin{array}{l}6 \\
8\end{array}$ & $\begin{array}{l}2 \\
3\end{array}$ & $\begin{array}{l}6 \\
2 \\
2\end{array}$ & 56 & 8 & & 3 \\
\hline & & 2 & (i) & 1 & 16 & 7 & 46 & 11 & 32 & & & 12 & 9 & 4 & 1 & & 8 & & \\
\hline “" & 16 & 2 & 29 & 0 & 16 & 7 & 46 & 10 & 10 & 17 & 0 & 13 & 1 & 2 & 2 & 57 & 3 & -0 & 3 \\
\hline & & 2 & 51 & 2 & 01 & 8 & 16 & 11 & & & 4 & 13 & 1 & 2 & 3 & & & & \\
\hline "t & 17 & $\begin{array}{l}3 \\
3\end{array}$ & $\begin{array}{l}1 . \\
3\end{array}$ & 3 & 16 & $\begin{array}{l}7 \\
9\end{array}$ & $\begin{array}{l}16 \\
16\end{array}$ & 12 & 2 & & 2 & 3 & $\begin{array}{l}6 \\
7\end{array}$ & $\begin{array}{l}3 \\
0\end{array}$ & $\begin{array}{l}9 \\
3\end{array}$ & 57 & 7 & -6 & 0 \\
\hline “ & 18 & 4 & 00 & $\dddot{3}$ & 01 & 9 & 01 & ii & 24 & 17 & 24 & 10 & 6 & 2 & $\frac{5}{7}$ & 58 & 2 & -11 & 7 \\
\hline & & 4 & 24 & 2 & 1 & 9 & 1 & 10 & & & & 1 & 2 & 3 & 0 & & - & & \\
\hline " & 19 & 4 & $4 !$ & 3 & 16 & 10 & 46 & 11 & & & & 11 & 3 & 4 & 5 & 58 & 7 & -16 & 8 \\
\hline & & 5 & 10 & 3 & 16 & ] 0 & 16 & 10 & & & 27 & 10 & 7 & 3 & 0 & & & & \\
\hline " & 20 & 5 & 4 & 3 & 46 & 9 & 16 & 10 & 30 & 16 & 00 & 10 & 8 & 5 & 5 & 59 & 1 & -20 & 9 \\
\hline " & 21 & $\begin{array}{l}6 \\
6\end{array}$ & $\begin{array}{l}1 \\
3\end{array}$ & $\begin{array}{c}3 \\
\ldots\end{array}$ & $\begin{array}{l}46 \\
\ldots\end{array}$ & $\cdots$ & $\ldots$ & 10 & 04 & $\cdots$ & $\cdots$ & 10 & 7 & $\cdots$ & $\cdots$ & & & -24 & \\
\hline & & 7 & 69 & $\ldots$ & $\ldots$ & $\dddot{11}$ & $\dddot{16}$ & $\cdots$ & $\begin{array}{l}\cdots \\
\cdots\end{array}$ & $\dddot{17}$ & $\dddot{36}$ & $\ldots$ & $\ldots$ & $\cdots$ & $\dddot{m}_{3}$ & 59 & 5 & & 1 \\
\hline “ & 22 & 7 & 40 & $\cdots$ & & $\ldots$ & $\ldots$ & $\ldots$ & $\cdots$ & & 0 & $\ldots$ & $\ldots$ & $\ldots$ & & 59 & 8 & -25 & 7 \\
\hline & & 8 & 10 & 7 & 46 & 4 & 16 & 12 & 06 & 21 & 0 & 9 & 1 & 4 & 7 & & & & \\
\hline "“ & 23 & 8 & 41 & 10 & & 2 & 4 & 14 & & & 0 & 10 & 7 & 4 & 1 & 59 & 9 & -25 & 6 \\
\hline & & 9 & & 8 & & 4 & 4 & 1 & & & & 8 & 9 & 5 & 7 & & & & \\
\hline " & 24 & 9 & & 9 & & 1 & 1 & 1 & & & & 10 & 3 & 3 & 3 & 59 & 9 & -23 & 7 \\
\hline & & 10 & & 10 & & 3 & 4 & & & & & 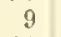 & 7 & 2 & 8 & & & & \\
\hline “" & 25 & 1 & 45 & 1 & & 3 & 4 & 1 & $0:$ & 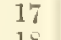 & 5 & 11 & 3 & 3 & 8 & 59 & 6 & -20 & 3 \\
\hline " & 26 & $\begin{array}{l}11 \\
11\end{array}$ & $\begin{array}{l}13 \\
41\end{array}$ & $\begin{array}{l}10 \\
12\end{array}$ & $\begin{array}{l}4 \\
4\end{array}$ & $\begin{array}{l}4 \\
4\end{array}$ & $\begin{array}{l}1 \\
4\end{array}$ & $\begin{array}{l}1 \\
1\end{array}$ & $\begin{array}{l}0 \\
3\end{array}$ & & $\begin{array}{l}0 \\
0\end{array}$ & $\begin{array}{l}10 \\
11\end{array}$ & $\begin{array}{l}4 \\
9\end{array}$ & $\frac{2}{1}$ & $\begin{array}{l}0 \\
0\end{array}$ & 59 & 2 & -15 & 7 \\
\hline & & $\ldots$ & & 11 & 4 & 6 & 0 & 1 & & & & 11 & 3 & 3 & 0 & & - & & \\
\hline “ & 27 & 0 & $0^{\prime}$ & 11 & & 5 & & & & & & & 7 & - 1 & 4 & 58 & 6 & -10 & 3 \\
\hline & & 0 & & 11 & & 6 & & & & & & & 3 & & $?$ & & & - & \\
\hline " & 8 & 0 & & 12 & 3 & 6 & 1 & 1 & 3 & & 4 & 13 & (1) & 0 & 1 & 57 & 9 & -4 & 5 \\
\hline & & 1 & 2 & 11 & & 7 & & 10 & 2 & & & 12 & 4 & 1 & 1 & & & & \\
\hline March & & 1 & & $\cdots$ & & 7 & & $\because$ & & & & & $\ldots$ & 0 & 5 & 57 & 1 & +1 & 4 \\
\hline$"$ & 2 & $\begin{array}{l}2 \\
2\end{array}$ & 0 & 1 & 3 & 8 & 4 & 11 & & & & 12 & 9 & 1 & 3 & & 4 & 7 & 0 \\
\hline & & 2 & 5 & 1 & & $\begin{array}{l}7 \\
9\end{array}$ & & $\begin{array}{l}1 \\
1\end{array}$ & & & & & $\begin{array}{l}0 \\
0\end{array}$ & $\begin{array}{l}2 \\
1\end{array}$ & $\begin{array}{l}5 \\
9\end{array}$ & 30 & & & \\
\hline$"$ & 3 & 3 & & 2 & & 8 & & 1 & & & & & 6 & 2 & 5 & 55 & 7 & +12 & 2 \\
\hline & & 3 & & 2 & & 9 & 0 & 1 & & & & 1 & 3 & 1 & 5 & & & & \\
\hline$"$ & 4 & 4 & & 3 & 13 & 7 & & & & & & & 1 & 3 & 9 & 55 & 0 & +16 & 7 \\
\hline & & 4 & & 2 & & 8 & & 10 & & & & 11 & & 1 & 0 & & & & \\
\hline ". & 5 & 4 & & 3 & 4 & 8 & & 11 & 2 & & & 9 & 2 & 3 & 1 & 54 & 6 & +20 & 5 \\
\hline “" & 6 & $\begin{array}{l}5 \\
5\end{array}$ & $\begin{array}{l}0 \\
3\end{array}$ & $\begin{array}{l}3 \\
4\end{array}$ & $\begin{array}{l}0 \\
1\end{array}$ & & & & & & & $\begin{array}{l}10 \\
10\end{array}$ & 1 & 3 & 9 & 54 & 3 & +5 & \\
\hline & & 5 & & 5 & 1 & & 3 & 1 & 4 & & & 9 & 6 & 4 & 1 & & & & \\
\hline “" & 7 & 6 & 22 & 4 & 49 & 10 & 49 & 10 & 53 & 16 & 53 & 8 & 8 & $\begin{array}{l}7 \\
5\end{array}$ & 8 & 54 & 2 & +25 & 3 \\
\hline & & 6 & 47 & $\cdots$ & $\cdots$ & $\cdots$ & $\cdots$ & $\ldots$ & $\cdots$ & $\cdots$ & $\ldots$ & $\cdots$ & $\ldots$ & $\cdots$ & $\ldots$ & & & & \\
\hline " & 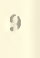 & $\begin{array}{l}8 \\
8\end{array}$ & $\begin{array}{l}03 \\
28\end{array}$ & $\ldots$ & $\ldots$ & $\ldots$ & $\ldots$ & $\ldots$ & $\ldots$ & $\ldots$ & $\ldots$ & $\ldots$ & $\ldots$ & $\cdots$ & $\ldots$ & & & +25 & 5 \\
\hline “ & 10 & 8 & 5 & $\dddot{9}$ & $\ddot{4}$ & $\dddot{4}$ & & $\dddot{13}$ & $\ddot{21}$ & & & $\because$ & $\begin{array}{c}\cdots \\
\vdots \\
\vdots\end{array}$ & $\dddot{3}$ & $\cdots$ & 54 & 9 & +23 & 8 \\
\hline & & 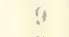 & & 10 & 50 & 4 & & 13 & 57 & 20 & 0 & 8 & 8 & 5 & 1 & & & & \\
\hline " & 11 & ! & 42 & 10 & 50 & 4 & 35 & 13 & 32 & $1 !$ & 42 & 10 & 6 & 5 & 4 & 6.5 & 4 & +21 & 0 \\
\hline & & 10 & us & $\ldots$ & $\ldots$ & $\ldots$ & $\ldots$ & $\ldots$ & $\cdots$ & $\cdots$ & $\cdots$ & $\ldots$ & $\cdots$ & $\ldots$ & & & & & \\
\hline "t & 1.1 & $\begin{array}{c}11 \\
11\end{array}$ & $\begin{array}{l}13 \\
06\end{array}$ & $\dddot{12}$ & $\ddot{21}$ & $\begin{array}{l}\ldots \\
\ldots\end{array}$ & $\begin{array}{l}\cdots \\
\cdots\end{array}$ & $\dddot{j}$ & 15 & $\begin{array}{l}\ldots \\
\ldots\end{array}$ & $\ldots$ & 11 & 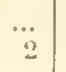 & $\begin{array}{l}\ldots \\
\ldots\end{array}$ & $\begin{array}{l}\ldots \\
\ldots\end{array}$ & & & +7 & 1 \\
\hline
\end{tabular}


TRCORD $\triangle \mathrm{ND}$ REDUCTION OF TIL TIDLS.

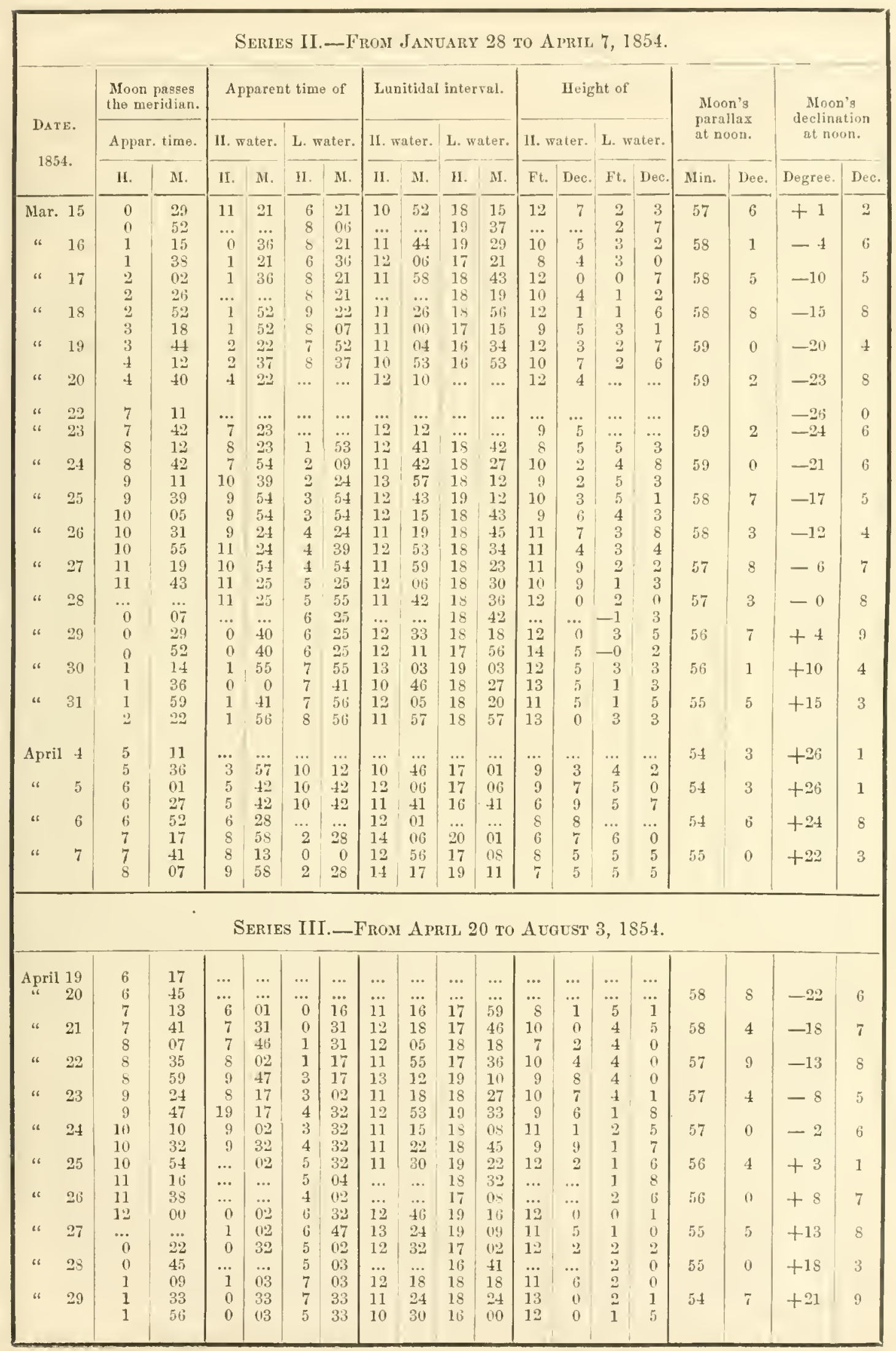




\begin{tabular}{|c|c|c|c|c|c|c|c|c|c|c|c|c|c|c|c|c|c|c|c|}
\hline \multicolumn{20}{|c|}{ Series III.-From $A$ pril 20 to $\Lambda$ ugust $3,1854}$. \\
\hline \multirow{3}{*}{\multicolumn{2}{|c|}{$\begin{array}{l}\text { DATE. } \\
1854 .\end{array}$}} & \multirow{2}{*}{\multicolumn{2}{|c|}{$\begin{array}{c}\text { Moon passes } \\
\text { the meridian. }\end{array}$}} & \multicolumn{4}{|c|}{ Apparent time of } & \multicolumn{4}{|c|}{ Lunitidal interraI. } & \multicolumn{4}{|c|}{ Height of } & \multirow{2}{*}{\multicolumn{2}{|c|}{$\begin{array}{l}\text { Moon's } \\
\text { parallax } \\
\text { at noon. }\end{array}$}} & \multirow{2}{*}{\multicolumn{2}{|c|}{$\begin{array}{l}\text { Moon's } \\
\text { decination } \\
\text { at noon. }\end{array}$}} \\
\hline & & & & \multicolumn{2}{|c|}{ I. water. } & \multicolumn{2}{|c|}{ L. water. } & \multicolumn{2}{|c|}{ II. water. } & L. wa & ater. & HI. $\pi$ & ater. & L. $\pi \mathrm{a}$ & ater. & & & & \\
\hline & & H. & M. & II. & M. & $\mathrm{H}$. & M. & II. & M. & H. & M. & Ft. & Dec. & Ft. & Dec. & Min. & Dec. & Degree. & Dec. \\
\hline April 3 & & 2 & 20 & 0 & 03 & 5 & 33 & 10 & 07 & 15 & 37 & 12 & 9 & 1 & 9 & 54 & 4 & +24 & 5 \\
\hline Nay & & $\frac{2}{3}$ & $\begin{array}{l}45 \\
09\end{array}$ & $\begin{array}{l}0 \\
1\end{array}$ & $\begin{array}{l}33 \\
18\end{array}$ & $\begin{array}{l}5 \\
9\end{array}$ & $\begin{array}{l}17 \\
03\end{array}$ & $\begin{array}{l}10 \\
10\end{array}$ & $\begin{array}{l}13 \\
33\end{array}$ & $\begin{array}{l}14 \\
18\end{array}$ & $\begin{array}{l}57 \\
18\end{array}$ & 11 & $\begin{array}{l}3 \\
6\end{array}$ & 1 & 7 & 54 & 2 & +26 & 0 \\
\hline May & & 3 & 34 & 0 & 33 & 7 & 03 & 9 & 24 & 15 & 54 & 9 & $\begin{array}{l}0 \\
4\end{array}$ & $\begin{array}{l}3 \\
3\end{array}$ & $\frac{9}{7}$ & 54 & 2 & +26 & 0 \\
\hline & & 3 & 59 & 2 & 33 & 9 & 03 & 10 & 59 & 17 & 29 & 12 & 4 & 1 & 8 & 54 & 2 & +20 & 3 \\
\hline & 3 & $\begin{array}{l}4 \\
4\end{array}$ & $\begin{array}{l}25 \\
50\end{array}$ & $\begin{array}{l}2 \\
3\end{array}$ & $\begin{array}{l}33 \\
49\end{array}$ & $\begin{array}{l}8 \\
9\end{array}$ & $\begin{array}{l}33 \\
03\end{array}$ & $\begin{array}{l}10 \\
11\end{array}$ & $\begin{array}{l}34 \\
23\end{array}$ & $\begin{array}{l}16 \\
16\end{array}$ & $\begin{array}{l}34 \\
34\end{array}$ & $\begin{array}{r}10 \\
9\end{array}$ & 3 & 4 & 3 & & & & \\
\hline & & 5 & 15 & $\begin{array}{l}0 \\
3\end{array}$ & 13 & $\begin{array}{l}9 \\
9\end{array}$ & 03 & 10 & 13 & $16^{\circ}$ & $\begin{array}{l}35 \\
13\end{array}$ & $\begin{array}{l}9 \\
9\end{array}$ & $\begin{array}{l}6 \\
1\end{array}$ & $\begin{array}{l}5 \\
6\end{array}$ & $\begin{array}{l}3 \\
5\end{array}$ & $5 t$ & 3 & +20 & 4 \\
\hline " & 4 & 5 & 39 & 4 & 33 & $\ldots$ & $\ldots$ & 11 & 18 & $\ldots$ & $\ldots$ & 10 & 5 & $\ldots$ & $\ldots$ & 54 & 6 & +23 & 4 \\
\hline & & 6 & 04 & $\cdots$ & $\cdots$ & $\cdots$ & $\cdots$ & $\cdots$ & $\cdots$ & $\cdots$ & $\cdots$ & $\cdots$ & $\cdots$ & $\cdots$ & $\cdots$ & & & & \\
\hline " & 6 & $\frac{7}{7}$ & $\begin{array}{l}14 \\
38\end{array}$ & $\begin{array}{l}\ldots \\
\ldots\end{array}$ & $\begin{array}{l}\ldots \\
\ldots\end{array}$ & $\begin{array}{l}\ldots \\
\ldots\end{array}$ & $\ldots$ & $\ldots$ & $\ldots$ & $\ldots$ & $\ldots$ & $\ldots$ & $\begin{array}{l}\ldots \\
\ldots\end{array}$ & $\ldots$ & $\ldots$ & 55 & 6 & +16 & 3 \\
\hline " & 7 & s & 02 & 6 & 34 & $\dddot{0}$ & 49 & $\dddot{10}$ & $\dddot{50}$ & $\dddot{17}$ & $\ddot{35}$ & $\dddot{8}$ & $\dddot{9}$ & $\ddot{6}$ & $\dddot{0}$ & 56 & 4 & +11 & 4 \\
\hline & & 8 & 25 & 9 & 34 & 2 & 04 & 13 & 32 & 18 & 26 & 7 & 9 & 3 & 6 & & & & \\
\hline " & 8 & 8 & $\begin{array}{l}48 \\
10\end{array}$ & 8 & 34 & 1 & 04 & 12 & 09 & 17 & 02 & 11 & 5 & 5 & 2 & 57 & 3 & +6 & 0 \\
\hline " & 9 & $\begin{array}{l}2 \\
9\end{array}$ & $\begin{array}{l}10 \\
33\end{array}$ & $\begin{array}{r}10 \\
8\end{array}$ & $\begin{array}{l}19 \\
34\end{array}$ & $\begin{array}{l}4 \\
2\end{array}$ & $\begin{array}{l}04 \\
04\end{array}$ & $\begin{array}{l}13 \\
11\end{array}$ & $\begin{array}{l}31 \\
24\end{array}$ & $\begin{array}{l}19 \\
17\end{array}$ & $\begin{array}{l}39 \\
16\end{array}$ & $\begin{array}{r}8 \\
11\end{array}$ & $\begin{array}{l}1 \\
3\end{array}$ & $\begin{array}{l}2 \\
4\end{array}$ & $\begin{array}{l}7 \\
4\end{array}$ & 58 & 1 & 0 & 0 \\
\hline & & 9 & $5 b^{\circ}$ & 9 & 0.4 & 3 & 19 & 11 & 31 & 18 & 09 & 9 & 4 & 1 & 8 & & & & \\
\hline “ & & 10 & 19 & 10 & 49 & 5 & 34 & 12 & 53 & 20 & 01 & 8 & 9 & 2 & 0 & 58 & 5 & -6 & 0 \\
\hline$" t$ & & 10 & 43 & 12 & 04 & 3 & 34 & 13 & 45 & 17 & 38 & 11 & 8 & 2 & 2 & & & & \\
\hline " & 11 & $\begin{array}{l}11 \\
11\end{array}$ & $\begin{array}{l}09 \\
35\end{array}$ & $\begin{array}{l}11 \\
10\end{array}$ & $\begin{array}{r}04 \\
+9\end{array}$ & 4 & 34 & 12 & $\begin{array}{l}21 \\
40\end{array}$ & $\begin{array}{l}18 \\
18\end{array}$ & $\begin{array}{l}15 \\
21\end{array}$ & $\begin{array}{l}10 \\
13\end{array}$ & 5 & 1 & 9 & 59 & $\bar{T}$ & -12 & 0 \\
\hline$"$ & 12 & 12 & UI & 12 & $\begin{array}{l}79 \\
04\end{array}$ & 5 & $\begin{array}{r}04 \\
49\end{array}$ & $\begin{array}{l}11 \\
12\end{array}$ & $\begin{array}{l}40 \\
29\end{array}$ & 18 & 40 & $\begin{array}{l}15 \\
11\end{array}$ & 0 & $\begin{array}{l}1 \\
1\end{array}$ & $\begin{array}{l}3 \\
0\end{array}$ & 60 & 2 & -17 & 4 \\
\hline & & 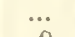 & 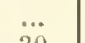 & 11 & 34 & 4 & 34 & 11 & 32 & 16 & 59 & 13 & 1 & 0 & 9 & & & & \\
\hline " & 13 & 0 & 30 & 10 & 49 & $\cdots$ & $\ldots$ & 10 & 19 & $\ldots$ & $\ldots$ & 12 & 4 & $\ldots$ & $\ldots$ & 60 & 5 & -22 & 0 \\
\hline " & 14 & $\begin{array}{l}1 \\
1\end{array}$ & $\begin{array}{l}00 \\
30\end{array}$ & $\begin{array}{l}11 \\
\ldots\end{array}$ & $\begin{array}{l}0.4 \\
\ldots\end{array}$ & $\begin{array}{r}5 \\
\ldots\end{array}$ & $\begin{array}{l}19 \\
\ldots\end{array}$ & 10 & 04 & $\begin{array}{l}16 \\
\ldots\end{array}$ & $\begin{array}{l}49 \\
\ldots\end{array}$ & 13 & $\begin{array}{c}6 \\
\ldots\end{array}$ & $\begin{array}{c}1 \\
\ldots\end{array}$ & $\begin{array}{c}3 \\
\ldots\end{array}$ & 60 & 6 & -25 & 0 \\
\hline & & 2 & 01 & "0 & 4 & $\because$ & 19 & $\dddot{10}$ & $\dddot{34}$ & 16 & $\dddot{49}$ & $\dddot{11}$ & $\ddot{3}$ & $\dddot{0}$ & $\ddot{8}$ & & 0 & & \\
\hline “ & 15 & 2 & 33 & 0 & 49 & 7 & 34 & 10 & 48 & 17 & 33 & 13 & 9 & 1 & 7 & 60 & 4 & -26 & 3 \\
\hline & & 3 & 05 & 1 & 49 & 8 & 19 & 11 & 16 & 17 & 46 & 12 & 3 & $\dot{0}$ & 5 & & & & \\
\hline “ & 16 & $\begin{array}{l}3 \\
4\end{array}$ & 38 & 0 & 49 & 8 & 04 & 9 & 44 & 16 & 59 & 12 & 4 & 1 & $\begin{array}{l}8 \\
0\end{array}$ & 60 & 0 & -25 & 8 \\
\hline$"$ & 17 & 4 & $\begin{array}{l}09 \\
41\end{array}$ & $\frac{2}{2}$ & $\begin{array}{l}34 \\
49\end{array}$ & $\begin{array}{l}7 \\
9\end{array}$ & $\begin{array}{l}19 \\
04\end{array}$ & $\begin{array}{l}10 \\
10\end{array}$ & $\begin{array}{l}56 \\
40\end{array}$ & $\begin{array}{l}15 \\
16\end{array}$ & $\begin{array}{l}41 \\
55\end{array}$ & $\begin{array}{l}10 \\
12\end{array}$ & $\begin{array}{l}4 \\
8\end{array}$ & $\begin{array}{l}2 \\
1\end{array}$ & $\begin{array}{l}3 \\
S\end{array}$ & 59 & 4 & -23 & 5 \\
\hline & & 5 & 10 & 2 & 49 & 8 & 19 & 10 & 08 & 15 & 38 & 10 & 6 & 2 & 0 & & & & \\
\hline$"$ & 18 & 5 & 40 & 3 & 49 & 10 & 49 & 10 & 39 & 17 & 39 & 12 & 5 & 1 & 8 & 58 & 8 & -19 & 8 \\
\hline & & 6 & 07 & 3 & 49 & 9 & 19 & 10 & 09 & 15 & 39 & 9 & 6 & 4 & 2 & & & & \\
\hline " & 19 & 6 & 3.4 & 5 & 19 & $\ldots$ & $\ldots$ & 11 & 12 & $\ldots$ & $\ldots$ & 11 & 7 & $\ldots$ & $\ldots$ & 58 & 1 & -15 & 0 \\
\hline "s & 20 & 6 & $\begin{array}{l}59 \\
24\end{array}$ & 6 & $\begin{array}{l}19 \\
79\end{array}$ & 0 & $\begin{array}{l}104 \\
34\end{array}$ & 11 & $\begin{array}{l}45 \\
20\end{array}$ & 17 & 57 & 10 & 2 & 3 & $\begin{array}{l}0 \\
2\end{array}$ & $5 m$ & 5 & -9 & 7 \\
\hline & & 7 & $\begin{array}{l}24 \\
47\end{array}$ & $\begin{array}{l}7 \\
8\end{array}$ & $\begin{array}{l}19 \\
34\end{array}$ & $\begin{array}{l}0 \\
3\end{array}$ & $\begin{array}{l}34 \\
04\end{array}$ & $\begin{array}{l}12 \\
13\end{array}$ & $\begin{array}{l}20 \\
10\end{array}$ & $\begin{array}{l}18 \\
20\end{array}$ & 0.5 & $\begin{array}{l}9 \\
8\end{array}$ & 7 & $\begin{array}{l}5 \\
3\end{array}$ & $\begin{array}{l}2 \\
8\end{array}$ & 57 & 5 & -9 & 7 \\
\hline " & 21 & 8 & 10 & 7 & 34 & 2 & 19 & 11 & 47 & 18 & 55 & 11 & 1 & 4 & 4 & 56 & 9 & -4 & 0 \\
\hline & & 8 & 32 & 8 & 34 & 2 & 34 & 12 & 24 & 18 & 47 & 8 & 6 & 3 & 7 & & & & \\
\hline " & 22 & 8 & 5.5 & 8 & 04 & 1 & 34 & 11 & 32 & 17 & 24 & 10 & 6 & 3 & 4 & 56 & 3 & +1 & 6 \\
\hline & & 9 & 16 & 9 & 04 & 3 & 19 & 12 & 09 & 18 & 47 & 9 & 3 & 2 & 5 & & & & \\
\hline “" & 23 & 9 & 38 & 8 & 04 & 3 & 0.4 & 10 & 48 & 18 & 09 & 11 & 3 & 3 & 5 & 55 & 8 & +7 & 3 \\
\hline & & 9 & 59 & 10 & 19 & 4 & 19 & 12 & 41 & 19 & 03 & 11 & 4 & 3 & 3 & & & & \\
\hline " & 24 & $\begin{array}{l}10 \\
10\end{array}$ & $\begin{array}{l}21 \\
43\end{array}$ & 8 & $4 s$ & 4 & 45 & 10 & 49 & 19 & 10 & 9 & 9 & 4 & 0 & 55 & 3 & +12 & 4 \\
\hline " & 25 & $\begin{array}{l}10 \\
11\end{array}$ & $\begin{array}{l}43 \\
05\end{array}$ & $\begin{array}{l}11 \\
10\end{array}$ & $\begin{array}{l}45 \\
18\end{array}$ & $\begin{array}{l}4 \\
5\end{array}$ & 18 & $\begin{array}{l}13 \\
11\end{array}$ & $\begin{array}{l}27 \\
35\end{array}$ & 1s & 19 & 12 & 9 & 2 & 0 & 54 & 9 & +17 & 1 \\
\hline & & 11 & 24 & 11 & $\begin{array}{l}18 \\
113\end{array}$ & $\begin{array}{l}5 \\
5\end{array}$ & $\begin{array}{l}03 \\
33\end{array}$ & 11 & $\begin{array}{l}35 \\
58\end{array}$ & $\begin{array}{l}18 \\
18\end{array}$ & $\begin{array}{l}42 \\
50\end{array}$ & $\begin{array}{l}10 \\
10\end{array}$ & 8 & $\begin{array}{l}2 \\
2\end{array}$ & $\begin{array}{l}11 \\
0\end{array}$ & 04 & & & 1 \\
\hline " & 26 & 11 & 51 & 12 & 45 & 5 & 03 & 13 & 17 & 17 & 58 & 9 & 7 & 2 & 7 & 54 & 6 & +20 & 9 \\
\hline & & & & 12 & 45 & i & 33 & 12 & 54 & 19 & 05 & 12 & 5 & 3 & 4 & & & & \\
\hline " & $2 \bar{\imath}$ & 0 & 15 & 10 & 48 & 6 & 18 & 10 & 33 & 18 & 27 & 9 & 7 & 2 & 4 & 5.4 & 3 & +23 & 9 \\
\hline "6 & 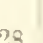 & 0 & 39 & $\cdots$ & $\dddot{3}$ & 4 & 18 & i. & $\dddot{r}$ & 16 & 0.3 & $\dddot{10}$ & $\cdots$ & 0 & 5 & 51 & 1 & +25 & 7 \\
\hline 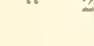 & 29 & 1 & $\begin{array}{l}14 \\
29\end{array}$ & 0 & $\begin{array}{l}33 \\
103\end{array}$ & $\begin{array}{l}6 \\
4\end{array}$ & $\begin{array}{l}4 n \\
4 y\end{array}$ & $\begin{array}{l}11 \\
10\end{array}$ & $\begin{array}{l}5.1 \\
59\end{array}$ & $\begin{array}{l}15 \\
15\end{array}$ & $\begin{array}{l}09 \\
44\end{array}$ & $\begin{array}{r}11 \\
8\end{array}$ & $\dot{7}$ & $\begin{array}{l}1 \\
2\end{array}$ & $\begin{array}{l}6 \\
3\end{array}$ & $3+4$ & 1 & 十20 & 8 \\
\hline " & 29 & 1 & 54 & 11 & 03 & 7 & 113 & 10 & 34 & 17 & 34 & 10 & 9 & 1 & 5 & 54 & 0 & $+2 i$ & 3 \\
\hline & & 2 & 19 & 0 & 44 & 7 & In & 10 & 54 & 17 & 24 & 8 & 5 & 1 & 6 & & & & \\
\hline " & 30 & 2 & 44 & $\frac{1}{2}$ & 0.3 & 8 & $\begin{array}{l}18 \\
48\end{array}$ & 10 & $\begin{array}{l}44 \\
34\end{array}$ & 17 & $\begin{array}{l}59 \\
04\end{array}$ & 12 & $\begin{array}{l}0 \\
5\end{array}$ & $\begin{array}{l}2 \\
2\end{array}$ & $\begin{array}{l}5 \\
5 \\
5\end{array}$ & 54 & 0 & +25 & S \\
\hline " & 31 & $\begin{array}{l}3 \\
3\end{array}$ & $\begin{array}{l}0.9 \\
34\end{array}$ & $\begin{array}{l}2 \\
3\end{array}$ & 18 & $\begin{array}{l}6 \\
9\end{array}$ & $\begin{array}{l}48 \\
18\end{array}$ & $\begin{array}{l}11 \\
12\end{array}$ & $\begin{array}{l}34 \\
09\end{array}$ & $\begin{array}{l}16 \\
14\end{array}$ & $\begin{array}{l}104 \\
09\end{array}$ & 11 & 5 & 2 & 5 & 54 & 2 & +24 & 1 \\
\hline & & 3 & 59 & 2 & 33 & s & 33 & 10 & 59 & 16 & 59 & 8 & 5 & 3 & 5 & & & & \\
\hline Juno & 1 & 4 & 22 & 2 & $1 \mathrm{~s}$ & 9 & 47 & 10 & 19 & 17 & 48 & 11 & 0 & 4 & 0 & 54 & 5 & +21 & 4 \\
\hline & & 4 & 47 & 4 & 17 & 10 & $(12$ & 11 & 55 & 17 & 411 & 8 & 0 & 3 & 6 & & & & \\
\hline
\end{tabular}




\begin{tabular}{|c|c|c|c|c|c|c|c|c|c|c|c|c|c|c|c|c|c|c|c|}
\hline \multicolumn{20}{|c|}{ Series III.-Tron 1 priL 20 to A lgust $3,1854}$. \\
\hline \multirow{3}{*}{\multicolumn{2}{|c|}{$\begin{array}{l}\text { DATE. } \\
1854 .\end{array}$}} & \multirow{2}{*}{\multicolumn{2}{|c|}{$\begin{array}{c}\text { Mloon passes } \\
\text { the meridian. } \\
\text { Appar. time. }\end{array}$}} & \multicolumn{4}{|c|}{ Apparent time of } & \multicolumn{4}{|c|}{ Lunitidal interval. } & \multicolumn{4}{|c|}{ Ileight of } & \multirow{2}{*}{\multicolumn{2}{|c|}{$\begin{array}{l}\text { Nloon's } \\
\text { parallax } \\
\text { at noon. }\end{array}$}} & \multirow{2}{*}{\multicolumn{2}{|c|}{$\begin{array}{l}\text { Moon's } \\
\text { declination } \\
\text { at noon. }\end{array}$}} \\
\hline & & & & \multicolumn{2}{|c|}{ II. water. } & \multicolumn{2}{|c|}{ L. water. } & 11. $w$ & ater. & L. W & ater. & II. WF & ater. & L. $w$ & ater. & & & & \\
\hline & & 11. & MI. & 11. & M. & 11 . & M. & 11. & M. & II. & M. & Ft. & Dee. & Ft. & Duc. & Min. & Doc. & Degree. & Dec. \\
\hline June & 2 & 5 & 09 & 4 & 02 & 10 & 32 & 11 & 15 & 17 & 45 & 9 & 8 & 3 & 7 & 54 & 9 & +17 & 6 \\
\hline & & 5 & 32 & 4 & 47 & 10 & 17 & 11 & 38 & 17 & 08 & 7 & 8 & 4 & 8 & & & & \\
\hline & 3 & $\begin{array}{l}5 \\
6\end{array}$ & $\begin{array}{l}54 \\
16\end{array}$ & $\dddot{4}$ & $\dddot{02}$ & $\dddot{10}$ & $\dddot{02}$ & $\dddot{10}$ & $\dddot{08}$ & $\dddot{16}$ & $\ddot{0 s}$ & $\begin{array}{l}9 \\
8\end{array}$ & 6 & $\begin{array}{l}4 \\
5\end{array}$ & $\begin{array}{l}5 \\
2 \\
2\end{array}$ & 55 & 5 & +13 & 1 \\
\hline " & 4 & 6 & 38 & $\ldots$ & 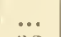 & $\ldots$ & $\ldots$ & $\ldots$ & $\ldots$ & $\ldots$ & $\ldots$ & 9 & $\begin{array}{l}1 \\
4\end{array}$ & $\begin{array}{c}5 \\
\ldots\end{array}$ & $\ldots$ & 56 & 3 & +8 & 0 \\
\hline & & 7 & 00 & 7 & 32 & 12 & 02 & 12 & 54 & 17 & 24 & 8 & 6 & 5 & 6 & 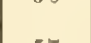 & 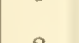 & & \\
\hline$"$ & 5 & 7 & 22 & 8 & $\begin{array}{l}132 \\
02\end{array}$ & $\because$ & $\dddot{39}$ & 13 & 02 & $\dddot{i 7}$ & 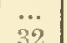 & 9 & 7 & $\cdots$ & $\cdots$ & 57 & 2 & +2 & 3 \\
\hline " & 6 & $\begin{array}{l}7 \\
8\end{array}$ & $\begin{array}{l}4.4 \\
07\end{array}$ & $\begin{array}{l}9 \\
9 \\
9\end{array}$ & $\begin{array}{l}02 \\
02\end{array}$ & 1 & 32 & $\begin{array}{l}13 \\
13\end{array}$ & $\begin{array}{l}411 \\
18\end{array}$ & 18 & 10 & 10 & $\begin{array}{l}2 \\
1\end{array}$ & $\begin{array}{l}4 \\
6\end{array}$ & 1 & 58 & 2 & -3 & 6 \\
\hline & & 8 & 30 & 8 & 02 & 2 & 32 & 11 & 55 & 18 & 48 & 10 & 4 & 4 & 0 & & & & \\
\hline$"$ & 7 & 8 & 54 & $?$ & 02 & 4 & 12 & 12 & 32 & 19 & 55 & 10 & 2 & 4 & 3 & 59 & 0 & -9 & 5 \\
\hline " & 8 & $\begin{array}{l}9 \\
9\end{array}$ & $\begin{array}{l}18 \\
4.4\end{array}$ & $\begin{array}{l}9 \\
8\end{array}$ & 02 & $\begin{array}{l}3 \\
3\end{array}$ & 112 & 12 & 08 & 18 & 32 & 11 & 1 & 3 & 4 & & & & \\
\hline & & 10 & 10 & 10 & $\begin{array}{l}01 \\
01\end{array}$ & $\begin{array}{l}3 \\
2\end{array}$ & $\begin{array}{l}47 \\
31\end{array}$ & $\begin{array}{l}10 \\
12\end{array}$ & $\begin{array}{l}43 \\
17\end{array}$ & $\begin{array}{l}17 \\
17\end{array}$ & $\begin{array}{l}53 \\
13\end{array}$ & $\begin{array}{r}9 \\
11\end{array}$ & $\begin{array}{l}3 \\
6\end{array}$ & $\begin{array}{l}4 \\
1\end{array}$ & $\begin{array}{l}0 \\
s\end{array}$ & 59 & 9 & -15 & 2 \\
\hline “ & 9 & 10 & 38 & $\ldots$ & $\ldots$ & $\ldots$ & $\ldots$ & $\ldots$ & & $\ldots$ & $\ldots$ & $\ldots$ & $\ldots$ & & $\ldots$ & 60 & 6 & -20 & 1 \\
\hline & & 11 & 07 & $\stackrel{9}{9}$ & 01 & 3 & 01 & 10 & 23 & 16 & 51 & 12 & 9 & 1 & 4 & & & & \\
\hline “" & 10 & 11 & 38 & 10 & 01 & 5 & 111 & 10 & 54 & 18 & 23 & 11 & 7 & 2 & 1 & 61 & 1 & -23 & $?$ \\
\hline & & & $\dddot{10}$ & $\begin{array}{l}13 \\
10\end{array}$ & 31 & 6 & $\theta 1$ & 13 & 53 & 18 & 54 & 11 & 4 & 0 & ij & & & & \\
\hline " & 11 & $\begin{array}{l}0 \\
0\end{array}$ & $\begin{array}{l}10 \\
43\end{array}$ & $\begin{array}{l}10 \\
13\end{array}$ & 31 & 6 & 01 & $\begin{array}{l}10 \\
10\end{array}$ & 21 & 18 & 23 & 9 & 4 & 1 & 8 & 61 & 2 & -26 & 0 \\
\hline " & 12 & 1 & 16 & 11 & $\begin{array}{l}01 \\
31\end{array}$ & $\begin{array}{l}6 \\
6\end{array}$ & $\begin{array}{l}31 \\
31\end{array}$ & $\begin{array}{l}12 \\
10\end{array}$ & $\begin{array}{l}18 \\
15\end{array}$ & $\begin{array}{l}18 \\
17\end{array}$ & $\begin{array}{l}21 \\
48\end{array}$ & $\begin{array}{l}13 \\
10\end{array}$ & $\begin{array}{l}2 \\
4\end{array}$ & 1 & 6 & 61 & 1 & -26 & 2 \\
\hline & & 1 & 49 & $\ldots$ & $\ldots$ & 6 & 31 & $\ldots$ & $\ldots$ & 17 & 15 & 12 & 2 & 0 & 4 & 8 & & & \\
\hline " & 13 & 3 & 21 & $\ddot{i}$ & $\dddot{00}$ & 8 & 30 & $\ldots$ & $\dddot{30}$ & 18 & $\begin{array}{l}41 \\
20\end{array}$ & $\ldots$ & $\cdots$ & 0 & 2 & 60 & 6 & -24 & 5 \\
\hline " & 14 & $\begin{array}{l}3 \\
3\end{array}$ & $\begin{array}{l}52 \\
24\end{array}$ & $\begin{array}{l}1 \\
1\end{array}$ & $\begin{array}{l}00 \\
30\end{array}$ & $\begin{array}{l}8 \\
7\end{array}$ & $\begin{array}{l}110 \\
30\end{array}$ & $\begin{array}{l}10 \\
10\end{array}$ & $\begin{array}{l}39 \\
38\end{array}$ & $\begin{array}{l}17 \\
16\end{array}$ & $\begin{array}{l}39 \\
38\end{array}$ & $\begin{array}{l}10 \\
12\end{array}$ & $\begin{array}{l}3 \\
1\end{array}$ & 1 & 1 & & 0 & & \\
\hline & 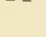 & 3 & 53 & 0 & 30 & 8 & 00 & 9 & 06 & 16 & 36 & 10 & 3 & 1 & $\begin{array}{l}6 \\
3\end{array}$ & 60 & 0 & -21 & 0 \\
\hline$"$ & 15 & 4 & 23 & 2 & 30 & 9 & 100 & 10 & 37 & 17 & 07 & 12 & 3 & 1 & 3 & 59 & 2 & -16 & 5 \\
\hline & & 4 & 49 & 1 & 30 & 8 & $(10$ & 9 & 07 & 15 & 37 & 8 & 5 & 2 & 3 & & & & \\
\hline " & 16 & 5 & 16 & 4 & 0 & 9 & 45 & 11 & 11 & 16 & 56 & 11 & 7 & 2 & 5 & 58 & 3 & -11 & 2 \\
\hline & & 5 & 40 & 2 & 0 & 9 & 00 & 8 & 44 & 15 & 44 & 8 & 9 & 3 & 7 & & & & \\
\hline “" & 17 & 6 & 04 & 3 & 0 & 9 & 00 & 9 & 20 & 15 & 20 & 11 & 4 & 2 & 8 & 57 & 4 & -5 & 4 \\
\hline " & & 6 & 27 & 2 & 30 & 11 & 29 & 8 & 26 & 17 & 25 & 9 & 3 & 4 & 6 & & & & \\
\hline & 18 & $\frac{6}{7}$ & $\begin{array}{l}49 \\
11\end{array}$ & $\begin{array}{l}4 \\
4\end{array}$ & 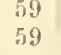 & $\begin{array}{l}11 \\
11\end{array}$ & $\begin{array}{l}29 \\
59\end{array}$ & $\begin{array}{l}10 \\
10\end{array}$ & $\begin{array}{l}32 \\
10\end{array}$ & $\begin{array}{l}17 \\
17\end{array}$ & $\begin{array}{l}122 \\
10\end{array}$ & $\begin{array}{l}10 \\
10\end{array}$ & $\begin{array}{l}2 \\
5\end{array}$ & $\begin{array}{l}3 \\
5\end{array}$ & $\begin{array}{l}2 \\
6\end{array}$ & $56^{\circ}$ & $i$ & +0 & 4 \\
\hline " & 19 & 7 & 33 & 5 & 59 & $\ldots$ & & 10 & ts & $\ldots$ & $\ldots$ & 10 & 5 & $\ldots$ & $\ldots$ & 56 & 0 & +6 & 0 \\
\hline & & 7 & 54 & 7 & 29 & 1 & 29 & 11 & 56 & $1 \mathrm{~s}$ & 15 & 10 & (1 & 4 & 0 & & & & \\
\hline “ & 20 & 8 & 16 & 7 & 59. & 2 & 14 & 12 & 05 & 18 & 41 & 9 & 9 & 5 & 2 & 55 & 4 & +11 & 3 \\
\hline " & & 8 & 37 & 9 & $29^{\circ}$ & 3 & 29 & 13 & 13 & 19 & 35 & 10 & 5 & 4 & 4 & & & & 0 \\
\hline " & 21 & $\begin{array}{l}8 \\
9\end{array}$ & $\begin{array}{l}59 \\
22\end{array}$ & $\begin{array}{l}8 \\
9\end{array}$ & $\begin{array}{l}59 \\
29\end{array}$ & $\frac{2}{2}$ & $\begin{array}{l}44 \\
59\end{array}$ & $\begin{array}{l}13 \\
12\end{array}$ & $\begin{array}{l}22 \\
30\end{array}$ & $\begin{array}{l}18 \\
18\end{array}$ & $\begin{array}{l}28 \\
22\end{array}$ & $\begin{array}{r}9 \\
13\end{array}$ & $\begin{array}{l}4 \\
0\end{array}$ & $\begin{array}{l}5 \\
3\end{array}$ & 8 & 54 & 9 & +16 & 0 \\
\hline " & 22 & 9 & 44 & 9 & $2 s$ & 2 & 58 & 12 & 06 & 17 & 59 & 9 & 9 & 2 & 9 & 54 & 5 & +20 & 1 \\
\hline & & 10 & 07 & 10 & 13 & 4 & 58 & 12 & 29 & 19 & 36 & 10 & 6 & 3 & 3 & & & & \\
\hline " & 23 & 10 & 31 & 9 & 28 & 3 & 58 & 11 & 21 & $1 \mathrm{~S}$ & 14 & s & 5 & 3 & 6 & 54 & 3 & +23 & 2 \\
\hline "6 & & 10 & 55 & 11 & 28 & 5 & 43 & 12 & 57 & 19 & 36 & 11 & 0 & 2 & $?$ & & & & \\
\hline 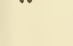 & 24 & 11 & 19 & 12 & 13 & 4 & 28 & 13 & 18 & 17 & 57 & 8 & 1 & 3 & 6 & 54 & 1 & +25 & 4 \\
\hline " & 25 & 11 & $\begin{array}{l}44 \\
\ldots\end{array}$ & $\begin{array}{l}10 \\
11\end{array}$ & $\begin{array}{l}58 \\
58\end{array}$ & $\begin{array}{l}5 \\
4\end{array}$ & 58 & $\begin{array}{l}11 \\
12\end{array}$ & $\begin{array}{l}39 \\
14\end{array}$ & $\begin{array}{l}18 \\
17\end{array}$ & $\begin{array}{l}33 \\
39\end{array}$ & $\begin{array}{r}11 \\
8\end{array}$ & $\begin{array}{l}3 \\
3\end{array}$ & $\stackrel{2}{2}$ & $\begin{array}{l}0 \\
4\end{array}$ & 54 & 0 & +20 & 3 \\
\hline & & 0 & 10 & & $\ldots$ & 6 & 58 & & . & 19 & 14 & & $\ldots$ & 3 & 1 & & & & \\
\hline " & 26 & 0 & 35 & 1 & 13 & 4 & 58 & 13 & 03 & 16 & 48 & 12 & 1 & 2 & 0 & 53 & 9 & +26 & 0 \\
\hline “ & 27 & 0 & 59 & 0 & 57 & 6 & 27 & 12 & 22 & 17 & 52 & 9 & 9 & 2 & 4 & & & & \\
\hline & 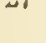 & $\begin{array}{l}1 \\
1\end{array}$ & $\begin{array}{l}24 \\
49\end{array}$ & ‥ & $\dddot{27}$ & $\begin{array}{l}4 \\
7\end{array}$ & $\begin{array}{l}57 \\
27\end{array}$ & $\dddot{11}$ & 우 & $\begin{array}{l}15 \\
1 \mathrm{~s}\end{array}$ & $\begin{array}{l}58 \\
13\end{array}$ & $\begin{array}{r}12 \\
9\end{array}$ & $\begin{array}{l}3 \\
8\end{array}$ & $\begin{array}{l}1 \\
3\end{array}$ & $\begin{array}{l}9 \\
0\end{array}$ & 54 & 0 & +24 & 6 \\
\hline “ & 28 & 2 & 13 & 0 & 57 & 7 & 57 & 11 & 08 & 18 & 08 & 11 & 4 & 3 & 2 & 54 & 2 & +23 & 1 \\
\hline & & 2 & 37 & 0 & 57 & 7 & 27 & 10 & 44 & 17 & 14 & 9 & 2 & 2 & 5 & & & & \\
\hline “ & 29 & 3 & 00 & 1 & 12 & 7 & 57 & 10 & 35 & 17 & 20 & 11 & 4 & 3 & 6 & 54 & 5 & $+1 s$ & 6 \\
\hline " & & 3 & 24 & 1 & 42 & 6 & 27 & 10 & 42 & 15 & 27 & 9 & 1 & 2 & 0 & & & & \\
\hline 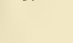 & 50 & $\begin{array}{l}3 \\
4\end{array}$ & 40 & 3 & 27 & 8 & $\begin{array}{l}57 \\
97\end{array}$ & 12 & 03 & 17 & 33 & 12 & 3 & 3 & 9 & 54 & 9 & +14 & 3 \\
\hline July & 1 & $\begin{array}{l}4 \\
4\end{array}$ & $\begin{array}{l}08 \\
30\end{array}$ & 4 & 42 & 9 & 57 & $\begin{array}{r}8 \\
12\end{array}$ & 34 & $\begin{array}{l}15 \\
17\end{array}$ & $\begin{array}{l}41 \\
49\end{array}$ & 11 & 6 & $\begin{array}{l}3 \\
2\end{array}$ & $\begin{array}{l}8 \\
6\end{array}$ & 55 & 5 & +9 & 4 \\
\hline & & 4 & 52 & 4 & 57 & 8 & 57 & 12 & 27 & 16. & 27 & 9 & 3 & 5 & 0 & & & & \\
\hline$"$ & 2 & 5 & 12 & 3 & 26 & 10 & 41. & 10 & 34 & 17 & $4 ?$ & 11 & 7 & 2 & 2 & 56 & 2 & +4 & 0 \\
\hline "6 & & 5 & 33 & 5 & 20 & 11 & 26 & 12 & 14 & 18 & 14 & 7 & 3 & 3 & 8 & & & & \\
\hline 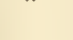 & 3 & 5 & $\begin{array}{l}55 \\
17\end{array}$ & 4 & 11. & 10 & 20 & 111 & 38 & 116 & 53 & 9 & 1 & 4 & $\varepsilon$ & 57 & 0 & -1 & 7 \\
\hline “ & 4 & 6 & $\begin{array}{l}17 \\
39\end{array}$ & b & $\begin{array}{l}26 \\
41\end{array}$ & $\begin{array}{l}10 \\
11\end{array}$ & $\begin{array}{l}56 \\
11\end{array}$ & $\begin{array}{l}11 \\
12\end{array}$ & $\begin{array}{l}21 \\
24\end{array}$ & $\begin{array}{l}17 \\
16\end{array}$ & $\begin{array}{l}01 \\
54\end{array}$ & 10 & $\begin{array}{l}8 \\
2\end{array}$ & $\begin{array}{l}6 \\
4\end{array}$ & $\begin{array}{l}9 \\
4\end{array}$ & 57 & $?$ & -7 & 5 \\
\hline & & 7 & 02 & 6 & 56 & $\ldots$ & $\ldots$ & 12 & 17 & $\ldots$ & $\ldots$ & 9 & 8 & $\ldots$ & $\ldots$ & & & & \\
\hline
\end{tabular}




\begin{tabular}{|c|c|c|c|c|c|c|c|c|c|c|c|c|c|c|c|c|c|c|c|}
\hline \multicolumn{20}{|c|}{ Series III._Frov A priL 20 to A ugust 3, 1854.} \\
\hline \multirow{3}{*}{\multicolumn{2}{|c|}{$\begin{array}{l}\text { DAte. } \\
1854 .\end{array}$}} & \multirow{2}{*}{\multicolumn{2}{|c|}{$\begin{array}{c}\begin{array}{c}\text { Moon passes } \\
\text { the meridian. }\end{array} \\
\text { Appar. time. }\end{array}$}} & \multicolumn{4}{|c|}{ Apparent time of } & \multicolumn{4}{|c|}{ Lunitidal interral. } & \multicolumn{4}{|c|}{ Height of } & \multirow{2}{*}{\multicolumn{2}{|c|}{$\begin{array}{l}\text { Moon's } \\
\text { parallax } \\
\text { at noon. }\end{array}$}} & \multirow{2}{*}{\multicolumn{2}{|c|}{$\begin{array}{l}\text { Moon's } \\
\text { declination } \\
\text { at noon. }\end{array}$}} \\
\hline & & & & \multicolumn{2}{|c|}{ F. water. } & \multicolumn{2}{|c|}{ L. water. } & \multicolumn{2}{|c|}{ H. water. } & L. $w \varepsilon$ & ater. & H. ws & ater. & L. wa & ater. & & & & \\
\hline & & Н. & M. & H. & M. & H. & M. & H. & M. & н. & M. & Ft. & Dec. & Ft. 1 & Dec. & Min. & Dec. & Degree. & Dec. \\
\hline July & 5 & 7 & 26 & 6 & 56 & 0 & 26 & 11 & 54 & 17 & 47 & 10 & 1 & 6 & 5 & 58 & 8 & -13 & 1 \\
\hline & & 7 & 51 & 7 & 56 & 1 & 26 & 12 & 30 & 18 & 24 & 10 & 6 & 4 & 3 & & & & \\
\hline & & 8 & 18 & 7 & 41 & 1 & 56 & 11 & 50 & 18 & 30 & 9 & 4 & 6 & 0 & 59 & 7 & -18 & 3 \\
\hline " & 7 & $\begin{array}{l}8 \\
8\end{array}$ & $\begin{array}{l}45 \\
14\end{array}$ & 8 & $\begin{array}{l}56 \\
55\end{array}$ & $\begin{array}{l}1 \\
2\end{array}$ & $\begin{array}{l}26 \\
55\end{array}$ & $\begin{array}{l}12 \\
11\end{array}$ & $\begin{array}{l}35 \\
10\end{array}$ & $\begin{array}{l}17 \\
18\end{array}$ & $\begin{array}{l}35 \\
37\end{array}$ & $\begin{array}{r}11 \\
9\end{array}$ & $\begin{array}{l}2 \\
2\end{array}$ & $\begin{array}{l}3 \\
5\end{array}$ & $\begin{array}{l}9 \\
6\end{array}$ & 60 & 5 & -22 & 5 \\
\hline & & 9 & 43 & $\ldots$ & $\ldots$ & 3 & 10 & $\ldots$ & $\ldots$ & 18 & 25 & $\ldots$ & $\ldots$ & 3 & 0 & 0 & 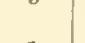 & 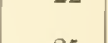 & 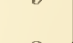 \\
\hline “ & 8 & 10 & 15 & $\ldots$ & $\cdots$ & $\cdots$ & $\cdots$ & $\ldots$ & $\cdots$ & $\cdots$ & $\cdots$ & $\cdots$ & $\cdots$ & $\cdots$ & $\ldots$ & 61 & 1 & -25 & 3 \\
\hline " & 9 & $\begin{array}{l}10 \\
11\end{array}$ & $\begin{array}{l}47 \\
20\end{array}$ & $\dddot{10}$ & $\dddot{40}$ & $\dddot{4}$ & $\dddot{40}$ & $\dddot{11}$ & $\dddot{53}$ & $\dddot{18}$ & $\ddot{25}$ & $\ddot{s}$ & $\ddot{5}$ & $\ddot{2}$ & $\cdots$ & 61 & 4 & -26 & 3 \\
\hline & & 11 & 54 & 11 & 40 & 4 & 25 & 12 & 211 & 17 & 38 & 12 & 5 & $\overrightarrow{1}$ & 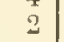 & 01 & 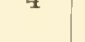 & -0 & 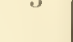 \\
\hline “ & 10 & $\ldots$ & & 12 & 55 & 5 & 25 & 12 & 51 & 18 & 05 & 8 & 4 & 2 & 2 & 61 & 4 & -25 & 4 \\
\hline “ & 11 & $\begin{array}{l}0 \\
1\end{array}$ & $\begin{array}{l}27 \\
00\end{array}$ & $\begin{array}{l}11 \\
12\end{array}$ & $\begin{array}{l}55 \\
25\end{array}$ & 5 & $\begin{array}{l}10 \\
25\end{array}$ & 11 & 28 & 17 & $\begin{array}{l}20 \\
58\end{array}$ & 13 & 6 & 1 & 2 & fi & 0 & & 5 \\
\hline & 11 & 1 & 31 & 12 & 55 & $\begin{array}{l}6 \\
6\end{array}$ & $\begin{array}{l}20 \\
10\end{array}$ & 11 & 24 & 17 & $\begin{array}{l}58 \\
10\end{array}$ & $\begin{array}{l}10 \\
13\end{array}$ & $\begin{array}{l}1 \\
3\end{array}$ & $\begin{array}{l}1 \\
1\end{array}$ & $\begin{array}{l}5 \\
2\end{array}$ & 61 & 0 & -22 & 5 \\
\hline “" & 12 & 2 & 02 & 11 & 40 & 6 & 55 & 9 & 38 & 17 & 24 & 10 & 1 & 1 & 0 & 60 & 3 & -18 & 3 \\
\hline & & $\frac{2}{3}$ & 31 & $\cdots$ & $\ldots$ & 7 & 10 & $\ldots$ & $\dddot{3}$ & 17 & 08 & $\ldots$ & $\cdots$ & 1 & 2 & & & & \\
\hline " & 13 & $\begin{array}{l}3 \\
3\end{array}$ & $\begin{array}{l}110 \\
26\end{array}$ & $\begin{array}{l}1 \\
1\end{array}$ & $\begin{array}{l}10 \\
25\end{array}$ & 7 & $\begin{array}{l}411 \\
55\end{array}$ & $\begin{array}{l}10 \\
10\end{array}$ & $\begin{array}{l}39 \\
25\end{array}$ & $\begin{array}{l}17 \\
16\end{array}$ & $\begin{array}{l}09 \\
55\end{array}$ & $\begin{array}{l}13 \\
10\end{array}$ & $\begin{array}{l}6 \\
0\end{array}$ & $\begin{array}{l}1 \\
2\end{array}$ & $\begin{array}{l}0 \\
4\end{array}$ & 59 & 5 & -12 & 9 \\
\hline$"$ & 14 & 3 & 52 & 1 & 55 & 9 & 10 & 10 & 29 & 17 & 41 & 12 & 6 & 1 & s & 58 & 5 & -7 & 2 \\
\hline & & 4 & 15 & 2 & 24 & 8 & 54 & 10 & 32 & 17 & 02 & 9 & 2 & 2 & 5 & & & & \\
\hline “" & 15 & 4 & 39 & $\cdots$ & $\cdots$ & $\cdots$ & $\cdots$ & $\cdots$ & $\cdots$ & $\cdots$ & $\cdots$ & $\cdots$ & $\cdots$ & $\cdots$ & $\cdots$ & 57 & 6 & -1 & 2 \\
\hline$"$ & 17 & 6 & 31 & $\ldots$ & $\ldots$ & $\ldots$ & $\ldots$ & $\ldots$ & $\cdots$ & $\cdots$ & $\ldots$ & $\ldots$ & $\ldots$ & $\cdots$ & $\cdots$ & & & +10 & 2 \\
\hline “" & 18 & $\begin{array}{l}6 \\
7\end{array}$ & 5 & $\dddot{7}$ & $\dddot{2} 4$ & $\dddot{1}$ & $\ddot{2} \dot{q}$ & $\dddot{7}$ & $\ddot{3}$ & $\dddot{1 s}$ & $\dddot{53}$ & $\dddot{9}$ & $\dddot{m}_{2}$ & $\cdots$ & $\because$ & 55 & 3 & +15 & 1 \\
\hline " & 19 & $\frac{1}{7}$ & $\begin{array}{l}16 \\
38\end{array}$ & 8 & 54 & $\ldots$ & ... & 13 & 38 & 10 & $\ldots$ & 7 & 6 & 4 & $\begin{array}{l}8 \\
6\end{array}$ & 54 & 7 & +19 & 3 \\
\hline & & 8 & 01 & 8 & 39 & 1 & 24 & 13 & 01 & 18 & os & 9 & 3 & 4 & 4 & & & & 3 \\
\hline “ & 20 & 8 & 24 & 9 & 54 & 2 & 39 & 13 & 53 & 19 & 01 & $s$ & 7 & 5 & 6 & 54 & 4 & +22 & 6 \\
\hline & & $\cdots$ & $\cdots$ & $\cdots$ & $\ldots$ & 1 & 54 & $\ldots$ & $\cdots$ & 17 & 53 & $\ldots$ & ... & 4 & 5 & & & & \\
\hline$"$ & 27 & 2 & 04 & $\ldots$ & $\ldots$ & $\ldots$ & $\ldots$ & $\ldots$ & $\ldots$ & $\ldots$ & $\ldots$ & $\ldots$ & $\ldots$ & $\cdots$ & $\ldots$ & & & +15 & 3 \\
\hline & 28 & $\frac{2}{2}$ & $\begin{array}{l}26 \\
48\end{array}$ & $\dddot{1}_{1}$ & $\dddot{54}$ & $\dddot{7}$ & $\dddot{54}$ & $\dddot{11}$ & $\dddot{28}$ & $\dddot{17}$ & $\dddot{2}$ & $\dddot{8}$ & $\because 6$ & $\dddot{m}_{2}$ & $\ddot{0}$ & 55 & 2 & +10 & 4 \\
\hline " & 29 & 3 & 09 & 2 & 54 & 8 & 09 & 12 & 06 & 17 & 21 & 12 & 0 & 1 & 7 & 55 & 7 & +5 & 2 \\
\hline & & 3 & 30 & 3 & 54 & 8 & 54 & 12 & 45 & 17 & 45 & 11 & 3 & 3 & 2 & & & & \\
\hline " & 30 & 3 & 51 & 2 & 09 & $\delta$ & 24 & 10 & 39 & 16 & 54 & 11 & 1 & 3 & 2 & 56 & 3 & -0 & 4 \\
\hline$" 6$ & & 4 & 13 & 2 & 54 & 8 & 24 & 11 & 03 & 16 & 33 & 9 & 2 & 4 & 1 & -8 & & & \\
\hline 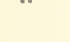 & 31 & 4 & $\begin{array}{l}34 \\
56 t\end{array}$ & $\because$ & $\dddot{39}$ & ${ }_{9}$ & $\dddot{39}$ & $\dddot{10}$ & $\ddot{0}$ & $\dddot{17}$ & $\ldots$ & $\begin{array}{r}10 \\
9\end{array}$ & $\begin{array}{l}6 \\
0\end{array}$ & $\begin{array}{l}4 \\
4 \\
4\end{array}$ & $\begin{array}{l}1 \\
8\end{array}$ & 56 & 9 & -6 & 1 \\
\hline Aug. & 1 & 5 & 19 & 3 & 24 & 16 & 39 & 10 & $2 s$ & 17 & 43 & 9 & 3 & 1 & 4 & 57 & 7 & -11 & 6 \\
\hline & & 5 & 43 & 4 & 39 & 9 & 54 & 11 & 20 & 16 & 35 & 10 & 5 & 6 & 1 & & & & \\
\hline$" 6$ & 2 & 6 & 117 & 4 & 54 & 11 & 09 & 11 & 11 & 17 & 26 & 10 & 5 & 4 & 5 & 58 & 5 & -16 & 8 \\
\hline ، & 3 & ij & $\begin{array}{l}32 \\
59\end{array}$ & $\begin{array}{l}5 \\
5\end{array}$ & $\begin{array}{l}54 \\
54\end{array}$ & $\dddot{11}$ & $\dddot{5 t}$ & $\begin{array}{l}11 \\
] 1\end{array}$ & $\begin{array}{l}47 \\
22\end{array}$ & $\ddot{19}$ & $\dddot{47}$ & $\begin{array}{l}? \\
8\end{array}$ & 5 & $\because$ & $\ddot{7}$ & 59 & 3 & -21 & 3 \\
\hline & & 7 & 27 & 8 & 24 & 1 & 09 & 13 & 25 & 18 & 37 & 9 & 4 & 3 & 6 & & & & \\
\hline & & & & SERI & $\mathrm{ES}$ & & & T & & IBER & & OCT & COBER & R2, & & & & & \\
\hline Sept. & . 7 & 0 & 23 & $\ldots$ & $\ldots$ & $\ldots$ & $\ldots$ & $\ldots$ & & $\ldots$ & $\ldots$ & & $\ldots$ & $\ldots$ & $\ldots$ & 59 & 3 & -5 & 7 \\
\hline & & 0 & 47 & 11 & 2 & $\cdots$ & $\ldots$ & 10 & 15 & $\dddot{10}$ & $\dddot{15}$ & 14 & 5 & & $\because$ & & & & \\
\hline 6 & 8 & $\begin{array}{l}1 \\
1\end{array}$ & 12 & 11 & 2 & 5 & 32 & 9 & 50 & 16 & 45 & 14 & 0 & -1 & 7 & 58 & 5 & +0 & 5 \\
\hline " & 9 & 2 & (11) & $\ddot{1}$ & 32 & $\begin{array}{l}8 \\
8\end{array}$ & 02 & $\dddot{11}$ & 56 & $\begin{array}{l}18 \\
18\end{array}$ & 26 & 13 & $\ddot{5}$ & 1 & 0 & 57 & 7 & +6 & 6 \\
\hline & & 2 & 2.1 & 1 & 32 & 7 & 32 & 11 & 31 & 17 & 31 & 14 & 0 & -0 & 5 & & & & \\
\hline “ & 10 & 2 & 47 & 1 & 03 & 7 & 33 & 10 & 39 & 17 & 09 & 13 & 0 & -1 & 11 & 56 & 9 & +12 & 3 \\
\hline & & 3 & 10 & 2 & 03 & 10 & 03 & 11 & 16 & 19 & 16 & 11 & 0 & 1 & 0 & & & & \\
\hline " & 11 & 3 & 33 & 2 & 33 & 8 & 33 & 11 & 23 & 17 & 2 & 11 & 0 & 0 & 0 & 56 & 1 & +17 & 2 \\
\hline & & 3 & 57 & $\cdots$ & $\ldots$ & 9 & U3 & $\ldots$ & & 17 & 30 & 10 & 5 & 1 & 5 & & & & \\
\hline " & 12 & 4 & 23 & 2 & 0.1 & 8 & 13 & 10 & 07 & 16 & $0 G_{3}$ & 14 & 0 & 0 & 0 & 55 & 4 & +21 & 2 \\
\hline & & 4 & 46 & 4 & 03 & 8 & 03 & 11 & 41 & 15 & 41 & 10 & 0 & "1 & 1) & & & & \\
\hline " & 13 & 5 & 11 & 3 & 04 & 7 & $(14$ & 10 & 18 & 14 & 18 & ]0 & 0 & $\begin{array}{l}0 \\
3\end{array}$ & $\begin{array}{l}0 \\
0 \\
0\end{array}$ & 54 & 8 & +24 & 2 \\
\hline & & 5 & 35 & 3 & 34 & 8 & 04 & 10 & 23 & 14 & 53 & 9 & $\begin{array}{l}0 \\
0\end{array}$ & $\begin{array}{c}3 \\
\ldots\end{array}$ & ... & 54 & 5 & +20 & 1 \\
\hline$"$ & 14 & i & $\begin{array}{l}00 \\
2.5\end{array}$ & $\begin{array}{r}2 \\
\ldots\end{array}$ & $\begin{array}{l}04 \\
\ldots\end{array}$ & $\begin{array}{l}\cdots \\
\ldots\end{array}$ & $\begin{array}{l}\ldots \\
\ldots\end{array}$ & $\begin{array}{c}0 \\
\ldots\end{array}$ & $\begin{array}{l}29 \\
\ldots\end{array}$ & $\begin{array}{l}\cdots \\
\ldots\end{array}$ & $\ldots$ & $\begin{array}{l}7 \\
\ldots\end{array}$ & $\begin{array}{c}0 \\
\ldots\end{array}$ & $\cdots$ & ... & 12 & & $T=$ & 1 \\
\hline " & 15 & ij & 52 & $\ldots$ & $\ldots$ & $\ldots$ & $\ldots$ & ... & $\ldots$ & ... & $\ldots$ & ... & $\ldots$ & $\ldots$ & ... & & & +26 & 7 \\
\hline & & 7 & 17 & $\ldots$ & $\ldots$ & $\ldots$ & $\ldots$ & $\ldots$ & $\ldots$ & $\cdots$ & $\cdots$ & $\ldots$ & $\cdots$ & $\ldots$ & $\cdots$ & & & & \\
\hline
\end{tabular}




\begin{tabular}{|c|c|c|c|c|c|c|c|c|c|c|c|c|c|c|c|c|c|c|c|}
\hline \multirow{3}{*}{\multicolumn{2}{|c|}{$\begin{array}{l}\text { DАте. } \\
1854 .\end{array}$}} & \multirow{2}{*}{\multicolumn{2}{|c|}{$\begin{array}{l}\text { Monn passes } \\
\text { the merillinn. } \\
\text { Appar, time. }\end{array}$}} & \multicolumn{4}{|c|}{$A_{\text {rintent time of }}$} & \multicolumn{4}{|c|}{ Lunitidal interval. } & \multicolumn{4}{|c|}{ Ileight of } & \multirow{2}{*}{\multicolumn{2}{|c|}{$\begin{array}{l}\text { Monn"s } \\
\text { farnlax } \\
\text { st noon. }\end{array}$}} & \multirow{2}{*}{\multicolumn{2}{|c|}{$\begin{array}{l}\text { Morn's } \\
\text { declination } \\
\text { at noon. }\end{array}$}} \\
\hline & & & & \multicolumn{2}{|c|}{ 11. water. } & \multicolumn{2}{|c|}{ L. water. } & \multicolumn{2}{|c|}{ II. water. } & I. $\mathrm{W}$ & ater & 11. w. & ater. & L. wa & ater. & & & & \\
\hline & & 11. & M. & II. & XI. & 11. & M. & II. & M. & 11. & il. & Ft. & Dec. & Ft. & Dec. & Min. & Dec. & Degree. & Dec. \\
\hline Sept & 16 & $\bar{z}$ & $\begin{array}{l}43 \\
1+5\end{array}$ & $\ldots$ & $\ldots$ & 11 & $3 j$ & $\ldots$ & $\ldots$ & 16 & & 10 & 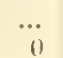 & 5 & 0 & 54 & 2 & +26 & 1 \\
\hline & 17 & 8 & 34 & 7 & ii.i & $\dddot{1}$ & $\dddot{3 i}$ & $\dddot{11}$ & $\dddot{58}$ & $\dddot{17}$ & $\dddot{3}$ & $\begin{array}{c}10 \\
\vdots\end{array}$ & 0 & $\dddot{\vdots}$ & $\cdots$ & 54 & 3 & +24 & 4 \\
\hline & 18 & 8 & $\begin{array}{ll}i s \\
2 y\end{array}$ & a & $11: i$ & 0 & 106 & 11 & 32 & 15 & sis & 10 & 4 & 5 & 0 & & & & \\
\hline & & $?$ & 22 & 1i & $\dddot{06}$ & $\begin{array}{l}4 \\
3\end{array}$ & $\begin{array}{l}0 \mathrm{Oi} \\
11 \mathrm{i} i\end{array}$ & $\ldots$ & $\dddot{4}$ & $\begin{array}{l}1: 1 \\
18\end{array}$ & $\begin{array}{l}32 \\
115\end{array}$ & $\begin{array}{r}9 \\
12\end{array}$ & $\begin{array}{l}0 \\
0\end{array}$ & $\begin{array}{l}5 \\
5\end{array}$ & $\begin{array}{l}0 \\
0\end{array}$ & 54 & 5 & +21 & 5 \\
\hline “ & 19) & 10) & 199 & $s$ & $3 i$ & 4 & $3 t i$ & 111 & 49 & 1. & 14 & 10 & 0 & 5 & (1) & 54 & 9 & +17 & $i$ \\
\hline & & 111 & 3.3 & 11 & 96 & (i) & Mi & 12 & 57 & 211 & 19 & 12 & 6 & 1 & (1) & & & & \\
\hline “ & 20 & $\begin{array}{l}10 \\
11\end{array}$ & $\begin{array}{l}5.5 \\
17\end{array}$ & $\dddot{11}$ & $\dddot{a r}$ & 4 & 117 & $\ldots$ & $\ldots$ & 18 & 58 & $\dddot{1}$ & $\ldots$ & 2 & (1) & 55 & 3 & +13 & 1 \\
\hline " & 21 & 11 & 39 & 11 & 07 & $\begin{array}{l}4 \\
5\end{array}$ & $\begin{array}{l}07 \\
07\end{array}$ & $\begin{array}{l}12 \\
11\end{array}$ & 12 & $\begin{array}{l}17 \\
19\end{array}$ & $\begin{array}{l}34 \\
12\end{array}$ & $\begin{array}{l}13 \\
11\end{array}$ & $\begin{array}{l}0 \\
5\end{array}$ & $\frac{2}{4}$ & $\begin{array}{l}0 \\
0\end{array}$ & $5 j$ & 8 & $+i$ & 8 \\
\hline & & & $\ldots$ & 11 & 07 & 3 & 07 & 11 & $2 s$ & 15 & 50 & 14 & 11 & 0 & 0 & & 8 & +1 & 8 \\
\hline " & 22 & 12 & & $\ldots$ & & 5 & 117 & $\ldots$ & $\ldots$ & 17 & 29 & ii. & $\ldots$ & 2 & (1) & 56 & 4 & +2 & 2 \\
\hline$"$ & 23 & $\begin{array}{l}0 \\
0\end{array}$ & $\begin{array}{l}2: 2 \\
45\end{array}$ & 0 & $\begin{array}{l}17 \\
\text { 115 }\end{array}$ & 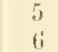 & 117 & $\begin{array}{l}12 \\
12\end{array}$ & $\begin{array}{l}07 \\
47\end{array}$ & $\begin{array}{l}17 \\
17\end{array}$ & $\begin{array}{l}117 \\
4 i\end{array}$ & 13 & $\begin{array}{l}0 \\
0\end{array}$ & 1 & 11 & & & & \\
\hline & & 1 & 07 & 0 & 08 & ii & 38 & 11 & 23 & 17 & 53 & 13 & $\begin{array}{l}0 \\
0\end{array}$ & $\begin{array}{r}-11 \\
0\end{array}$ & 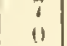 & 56 & 9 & -3 & 6 \\
\hline " & 24 & 1 & $2 ! !$ & 1 & $0 s$ & $i$ & 08 & 12 & (11 & 18 & OI & 11 & 0 & 2 & 0 & 57 & 4 & -9 & 5 \\
\hline & & 1 & 52 & 0 & 08 & 7 & 08 & 10 & 39 & 17 & 39 & 13 & 0 & $\overline{1}$ & (1) & & & & \\
\hline “ & 25 & 2 & 1.5 & 2 & 10. & 8 & 08 & 12 & 16 & 18 & 76 & 14 & 0 & 0 & 0 & 5 & 9 & -14 & 9 \\
\hline & & 2 & 39 & 1 & os & 8 & ns & 10 & 53 & 17 & 53 & 13 & $n$ & 0 & 0 & & & & \\
\hline “ & 26 & 3 & $0 b^{3}$ & 1 & 69 & 7 & 119 & 10 & 30 & 16 & 311 & 13 & "1 & 0 & 0 & 58 & 3 & -19 & $i$ \\
\hline " & $a$ & $\begin{array}{l}3 \\
3\end{array}$ & 32 & 2 & $0 ! 1$ & 8 & 199 & 11 & 0.3 & 17 & 03 & 13 & 0 & 0 & 0 & & if & -23 & 5 \\
\hline & -1 & 4 & $\begin{array}{l}59 \\
27\end{array}$ & $\frac{2}{3}$ & $\begin{array}{l}09 \\
09\end{array}$ & 8 & $\begin{array}{l}19 \\
19\end{array}$ & $\begin{array}{l}10 \\
11\end{array}$ & $\begin{array}{l}37 \\
10\end{array}$ & $\begin{array}{l}16 \\
16\end{array}$ & $\begin{array}{l}37 \\
10\end{array}$ & $\begin{array}{l}12 \\
13\end{array}$ & $\begin{array}{l}0 \\
0\end{array}$ & -1 & $\begin{array}{l}0 \\
0\end{array}$ & 58 & (i) & & 5 \\
\hline " & 28 & 4 & 56 & 3 & 09 & 8 & 09 & 10 & 42 & 15 & 42 & 13 & 0 & 1 & 0 & 58 & 9 & $-2 ;$ & 0 \\
\hline & & 5 & 25 & 4 & 0.9 & 9 & 09 & 11 & 13 & 16 & 13 & 12 & 0 & 2 & 0 & & & & \\
\hline " & 29 & 5 & 57 & 2 & 10 & 10 & 10 & 8 & 45 & 16 & 45 & 10 & 0 & 2 & 0 & 59 & 2 & $-2 ;$ & 8 \\
\hline & & ij & 28 & 4 & 10 & 9 & 10 & 10 & 13 & 15 & 13 & 11 & 5 & 3 & 0 & & & & \\
\hline " & 30 & $\frac{6}{7}$ & $\begin{array}{l}59 \\
30\end{array}$ & $\begin{array}{l}4 \\
5\end{array}$ & $\begin{array}{l}10 \\
40\end{array}$ & $\begin{array}{l}11 \\
12\end{array}$ & $\begin{array}{l}10 \\
10\end{array}$ & $\begin{array}{r}9 \\
10\end{array}$ & $\begin{array}{l}42 \\
41\end{array}$ & $\begin{array}{l}16 \\
17\end{array}$ & $\begin{array}{l}4: 2 \\
11\end{array}$ & $\begin{array}{r}9 \\
10\end{array}$ & $\begin{array}{l}0 \\
0\end{array}$ & $\begin{array}{l}3 \\
4\end{array}$ & $\begin{array}{l}0 \\
5\end{array}$ & 59 & 4 & -25 & $s$ \\
\hline Oet. & 1 & $\ldots$ & $\ldots$ & $\ldots$ & ... & ... & $\ldots$ & $\ldots$ & $\ldots$ & $\ldots$ & $\ldots$ & $\ldots$ & $\ldots$ & $\ldots$ & $\ldots$ & 59 & 5 & -23 & 3 \\
\hline " & 3 & 10 & 20 & & $\ldots$ & $\ldots$ & $\ldots$ & & $\ldots$ & $\ldots$ & $\ldots$ & $\ldots$ & $\ldots$ & $\ldots$ & $\ldots$ & & & -14 & 2 \\
\hline$"$ & 4 & 10 & 45 & 9 & 41 & $\ldots$ & $\ldots$ & 11 & 21 & $\ldots$ & $\ldots$ & 10 & 0 & $\ldots$ & $\ldots$ & 59 & 0 & $-\mathrm{s}$ & 3 \\
\hline " & 5 & 11 & 10 & 11 & 11 & 4 & 41 & 12 & 26 & 18 & 21 & 14 & 0 & 0 & $\begin{array}{l}0 \\
0\end{array}$ & 59 & & —.) & \\
\hline$"$ & 5 & $\begin{array}{l}11 \\
11\end{array}$ & $\begin{array}{l}36 \\
59\end{array}$ & 12 & $\begin{array}{l}12 \\
42\end{array}$ & 4 & $\begin{array}{l}11 \\
12\end{array}$ & $\begin{array}{l}13 \\
12\end{array}$ & 02 & $\begin{array}{l}17 \\
19\end{array}$ & $\begin{array}{l}26 \\
02\end{array}$ & $\begin{array}{l}13 \\
14\end{array}$ & $\begin{array}{l}0 \\
0\end{array}$ & $\begin{array}{l}-1 \\
-2\end{array}$ & $\begin{array}{l}2 \\
0\end{array}$ & 58 & 6 & -2 & 0 \\
\hline " & 6 & 0 & 22 & 12 & 12 & 5 & 12 & 12 & 13 & 17 & 36 & 12 & 0 & -1 & 0 & 57 & 9 & +4 & 3 \\
\hline & & $\ldots$ & & 11 & 12 & 7 & 12 & 10 & 50 & 19 & 13 & 14 & 0 & -0 & 5 & & & & \\
\hline " & 7 & 0 & $45^{\circ}$ & $\ldots$ & $\ldots$ & (j) & 42 & & & 19 & 20 & & $\ldots$ & 1 & 0 & 57 & 3 & +10 & 2 \\
\hline & & 1 & 09 & 0 & 12 & 5 & 42 & 11 & 20 & 16 & 56 & 14 & 0 & 0 & (1) & & & & \\
\hline “ & 8 & 1 & 33 & 0 & 12 & 8 & 12 & 11 & 13 & 19 & 03 & 14 & (1) & -1 & 0 & 50 & 6 & +15 & 5 \\
\hline " & 0 & 1 & 57 & 0 & 43 & 7 & 13 & 11 & 10 & 17 & 40 & 13 & 0 & 0 & 0 & & & & \\
\hline & $?$ & $\begin{array}{l}2 \\
2 \\
2\end{array}$ & 21 & 0 & 13 & $\dot{6}$ & 43 & 10 & 13 & 16 & 4i & 10 & 0 & 0 & 0 & 55 & 9 & +20 & 0 \\
\hline " & 10 & 3 & $\begin{array}{l}45 \\
10\end{array}$ & $\frac{2}{1}$ & $\begin{array}{l}13 \\
13\end{array}$ & $\frac{8}{6}$ & $\begin{array}{l}13 \\
13\end{array}$ & $\begin{array}{l}11 \\
10\end{array}$ & $\begin{array}{l}52 \\
29\end{array}$ & $\begin{array}{l}17 \\
15\end{array}$ & 52 & $\begin{array}{l}14 \\
13\end{array}$ & $\begin{array}{l}0 \\
0\end{array}$ & $\begin{array}{l}1 \\
0\end{array}$ & $\begin{array}{l}0 \\
0\end{array}$ & 55 & 3 & +23 & 4 \\
\hline & & 3 & 35 & 2 & 13 & 8 & 13 & 11 & 03 & 17 & 113 & 14 & 0 & 0 & 5 & & & & \\
\hline " & 11 & 4 & 00 & 1 & 13 & 8 & 13 & $?$ & 38 & 10 & 38 & 10 & 0 & 0 & 0 & 54 & 8 & +25 & 7 \\
\hline & & 4 & 25 & 1 & 13 & 8 & 13 & $?$ & 13 & 16 & 13 & 14 & 0 & 3 & 0 & & & & \\
\hline “ & 12 & 4 & 51 & 2 & 13 & 8 & 13 & 10 & 48 & 15 & 48 & 14 & 5 & 2 & 0 & 54 & 5 & +26 & 8 \\
\hline & & 5 & 17 & 3 & 44 & 9 & 14 & 10 & 53 & 16 & 23 & 14 & 0 & 4 & (1) & & & & \\
\hline " & 14 & 6 & 59 & $\ldots$ & $\ldots$ & $\ldots$ & $\ldots$ & $\ldots$ & $\ldots$ & $\ldots$ & $\ldots$ & $\ldots$ & $\ldots$ & $\ldots$ &.. & 54 & 3 & +25 & 3 \\
\hline " & 15 & 7 & $\begin{array}{l}23 \\
47\end{array}$ & $\ddot{s}_{s}$ & $\ddot{14}$ & 11 & $\begin{array}{l}14 \\
14\end{array}$ & $\dddot{12}$ & $\dddot{51}$ & $\begin{array}{l}16 \\
15\end{array}$ & $\begin{array}{l}16 \\
51\end{array}$ & 10 & 0 & 3 & 0 & 54 & 4 & $+2-2$ & 7 \\
\hline a & 16 & $\dot{8}$ & $\begin{array}{l}47 \\
111\end{array}$ & $\ldots$ & $\begin{array}{l}11 \\
\ldots\end{array}$ & $\begin{array}{l}11 \\
\ldots\end{array}$ & $\begin{array}{l}14 \\
\ldots\end{array}$ & $\ldots$ & ... & $\begin{array}{l}1.3 \\
\ldots\end{array}$ & $\begin{array}{l}51 \\
\ldots\end{array}$ & $\begin{array}{l}10 \\
\ldots\end{array}$ & $\begin{array}{c}0 \\
\ldots\end{array}$ & $\begin{array}{l}5 \\
\ldots\end{array}$ & $\begin{array}{c}0 \\
\ldots\end{array}$ & 54 & 8 & +19 & ร \\
\hline & & s & 34 & $\ldots$ & $\ldots$ & $\cdots$ & $\cdots$ & $\cdots$ & $\cdots$ & $\cdots$ & $\cdots$ & $\cdots$ & $\cdots$ & $\cdots$ & $\ldots$ & & & & \\
\hline$"$ & 17 & $\mathrm{~s}$ & 57 & 10 & 15 & 3 & 15 & 13 & 41 & 19 & 14 & 10 & 0 & 3 & 0 & 55 & 2 & +14 & $s$ \\
\hline " & 18 & 9 & 19 & 10 & 15 & 3 & 15 & 13 & 18 & 19 & 41 & 11 & 0 & 3 & 0 & & & & \\
\hline & 18 & $\begin{array}{r}9 \\
10\end{array}$ & $\begin{array}{l}41 \\
02\end{array}$ & 9 & 15 & 3 & 15 & 11 & 56 & 15 & 18 & 11 & 0 & 3 & 0 & 53 & $T$ & +9 & 7 \\
\hline “ & 19 & 10 & $\begin{array}{l}02 \\
24\end{array}$ & $\begin{array}{l}? \\
?\end{array}$ & $\begin{array}{l}15 \\
15\end{array}$ & $\begin{array}{l}3 \\
4\end{array}$ & $\begin{array}{l}45 \\
15\end{array}$ & $\begin{array}{l}11 \\
11\end{array}$ & $\begin{array}{l}34 \\
13\end{array}$ & $\begin{array}{l}18 \\
18\end{array}$ & $\begin{array}{l}26 \\
34\end{array}$ & $\begin{array}{l}12 \\
12\end{array}$ & $\begin{array}{l}0 \\
0\end{array}$ & $\begin{array}{l}2 \\
0\end{array}$ & $\begin{array}{l}0 \\
0\end{array}$ & 56 & 4 & +4 & 2 \\
\hline & & 10 & 46 & $\ldots$ & $\ldots$ & 3 & 45 & 11 & $\ldots$ & 17 & 43 & 11 & 0 & 1 & 0 & & & & \\
\hline " & 20 & 11 & as & 11 & 15 & 5 & 15 & 1 & 29 & 18 & 51 & 12 & 1) & 1 & (1) & 57 & 0 & -1 & G \\
\hline & & 11 & 30 & 12 & 15 & 4 & 15 & 13 & 07 & 17 & $2 ?$ & 11 & 11 & 11 & 0 & & & & \\
\hline " & 21 & 1I & 53 & 11 & 45 & 4 & 15 & 12 & 1.5 & 17 & $0 \overline{6}$ & 12 & 0 & 11 & (1) & 57 & 7 & -7 & 6 \\
\hline 4 & & & & 11 & 15 & 5 & 15 & 11 & 22 & 17 & 45 & 12 & (1 & 1) & 0 & -3 & 3 & & 1 \\
\hline & 23 & $\begin{array}{l}n \\
11\end{array}$ & $\begin{array}{l}17 \\
41\end{array}$ & $\begin{array}{l}11 \\
11\end{array}$ & $\begin{array}{l}45 \\
45\end{array}$ & $\begin{array}{l}4 \\
5\end{array}$ & 15 & 11 & 29 & $\begin{array}{l}16 \\
16\end{array}$ & $\begin{array}{l}22 \\
58\end{array}$ & $\begin{array}{l}13 \\
13\end{array}$ & $\begin{array}{l}11 \\
11\end{array}$ & $\begin{array}{l}11 \\
0\end{array}$ & $\begin{array}{l}0 \\
0\end{array}$ & is & 3 & -13 & 1 \\
\hline
\end{tabular}

$$
i
$$


The second form, or Table No. 2, for reduction of tides, is specially arranged to obtain the establishment and the half-montlily inequality in time and height. The first part is arranged in reference to the observed high waters; the second part, in reference to the low waters. 'That the inequality in time and lieight should also be made out from the low water, is specially important for stations where either the observations are of short extent, or else where difficulties tend to render the observations less accurate. The discussion of the low waters conld not be omitted in our case. The headings to the columns of Table No. 2, explain the arrangement sufficiently. 'The results from the upper and lower transit of the moon are kept separate. (It need hardly be remarked that, in certain months, the sun's or moon's lower transit can be observed at Tan liensselaer Harbor.) 
Table ron the RenUCtion of Tines.-No. 2.

Showing the Interval between the Apl. Time of the Moou's Superior Transit and the Time of High Water, and also the Ileights of IIigh Water, at Van Rensscher IIarbor, from Four Series of Ouservations made between October 10, 1853, and Octolser 22, 1854.

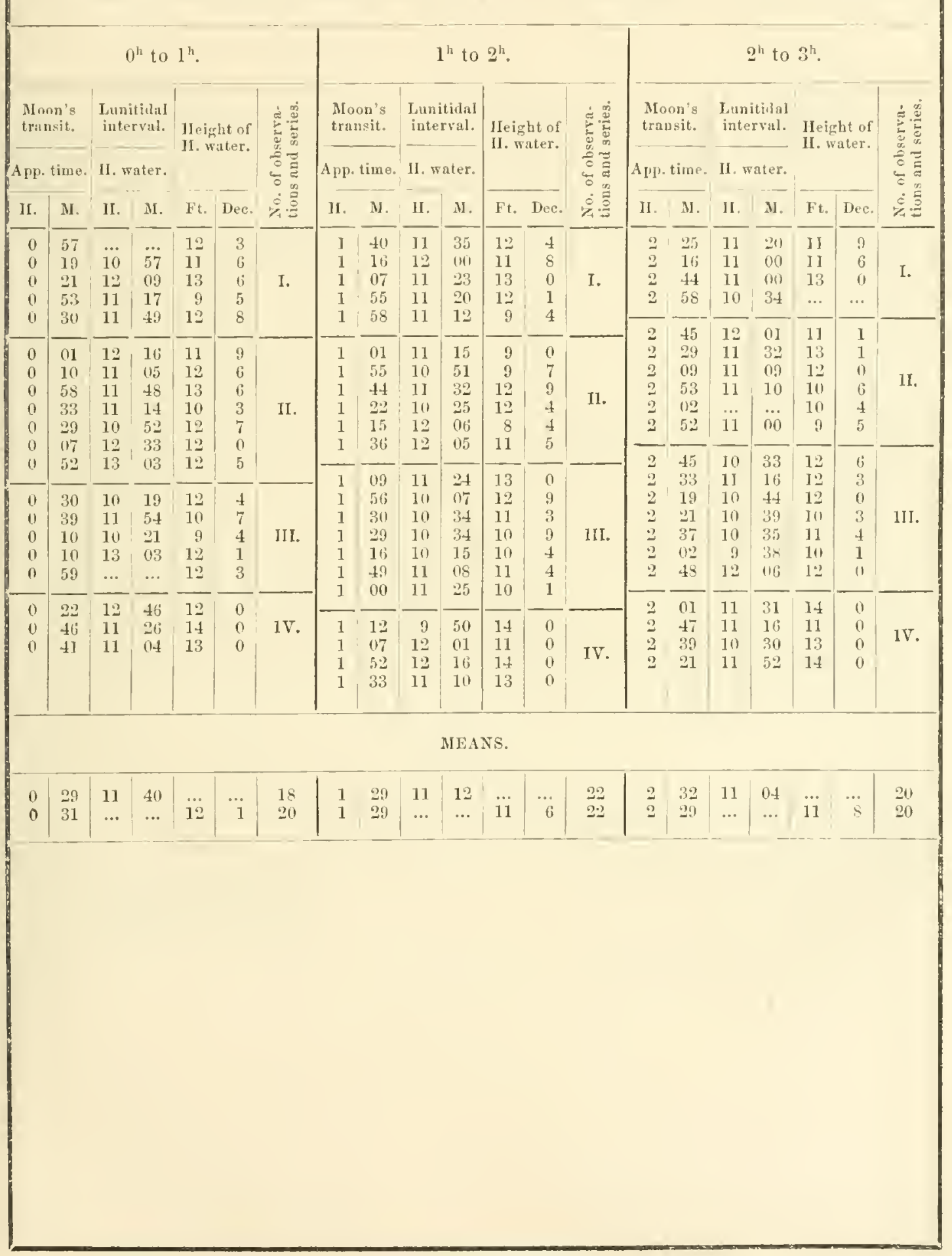


'TAble for the liedection of Tides - Yo. 2.

Showing the Interral between the App. Time of the Moon's Superior 'Transit and the Time of High Water, and also the Heights of IIigh Water, at Tan Reusselaer Harbor, from Four Series of Observations made between October 10, 1853, and October 22, 1854.

\begin{tabular}{|c|c|c|c|c|c|c|c|c|c|c|c|c|c|c|c|c|c|c|c|c|}
\hline \multicolumn{7}{|c|}{$3^{\mathrm{h}}$ to $4^{\mathrm{h}}$. } & \multicolumn{7}{|c|}{$4^{\text {h }}$ to $5^{\text {h }}$. } & \multicolumn{7}{|c|}{$5^{\mathrm{h}}$ to $6^{\mathrm{h}}$. } \\
\hline \multicolumn{2}{|c|}{$\begin{array}{l}\text { Mnon's } \\
\text { transit. }\end{array}$} & \multicolumn{2}{|c|}{$\begin{array}{l}\text { Lunitilal } \\
\text { interval. }\end{array}$} & \multicolumn{2}{|c|}{$\begin{array}{l}\text { Height of } \\
\text { H. water. }\end{array}$} & \multirow{2}{*}{ 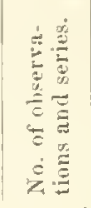 } & \multicolumn{2}{|c|}{$\begin{array}{c}\text { Moon } \\
\text { transit. } \\
\text { App. time. }\end{array}$} & \multicolumn{2}{|c|}{$\begin{array}{c}\text { Lunitidal } \\
\text { interval. } \\
\text { II. water. }\end{array}$} & \multicolumn{2}{|c|}{$\begin{array}{l}\text { Height of } \\
\text { II. water. }\end{array}$} & \multirow{2}{*}{ 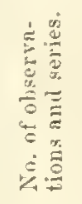 } & \multicolumn{2}{|c|}{$\begin{array}{c}\text { Moon's } \\
\text { transit. } \\
\text { Aprotime. }\end{array}$} & \multicolumn{2}{|c|}{$\begin{array}{l}\text { Lunitidal } \\
\text { interval. } \\
\text { II. water. }\end{array}$} & \multicolumn{2}{|c|}{$\begin{array}{l}\text { Height of } \\
\text { H. water. }\end{array}$} & \multirow{2}{*}{ 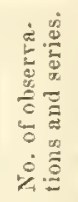 } \\
\hline II. & II. & II. & II. & Ft. & Dee. & & II. & M. & II. & M. & $\mathrm{Ft}$. & Dee. & & II. & II. & II. & MI. & Ft. & Dec. & \\
\hline $\begin{array}{l}3 \\
3 \\
3 \\
3 \\
3\end{array}$ & $\begin{array}{l}12 \\
19 \\
34 \\
13 \\
46\end{array}$ & $\begin{array}{r}12 \\
9 \\
10 \\
10 \\
11\end{array}$ & $\begin{array}{l}113 \\
57 \\
40 \\
51 \\
01\end{array}$ & $\begin{array}{l}11 \\
10 \\
11 \\
8 \\
10\end{array}$ & \begin{tabular}{l|l|}
1 & \\
1 & \\
5 & \\
3 & \\
4 &
\end{tabular} & & $\begin{array}{l}4 \\
4 \\
4 \\
4 \\
4 \\
4\end{array}$ & $\begin{array}{l}00 \\
51 \\
22 \\
24 \\
03 \\
32\end{array}$ & $\begin{array}{l}11 \\
11 \\
11 \\
10 \\
10 \\
11\end{array}$ & $\begin{array}{l}16 \\
25 \\
39 \\
20 \\
30 \\
14\end{array}$ & $\begin{array}{r}9 \\
10 \\
4 \\
10 \\
? \\
11\end{array}$ & $\begin{array}{l}9 \\
4 \\
1 \\
7 \\
1 \\
5\end{array}$ & & $\begin{array}{l}5 \\
5 \\
5 \\
5 \\
5 \\
5\end{array}$ & $\begin{array}{l}42 \\
23 \\
14 \\
00 \\
52 \\
17\end{array}$ & $\begin{array}{l}12 \\
10 \\
10 \\
10 \\
12 \\
11\end{array}$ & $\begin{array}{l}04 \\
23 \\
29 \\
09 \\
4.6 \\
14\end{array}$ & $\begin{array}{r}9 \\
8 \\
10 \\
6 \\
6 \\
11\end{array}$ & $\begin{array}{l}7 \\
2 \\
1 \\
1 \\
8 \\
6\end{array}$ & I. \\
\hline $\begin{array}{l}3 \\
3 \\
3 \\
3\end{array}$ & $\begin{array}{l}32 \\
14 \\
35 \\
41\end{array}$ & $\begin{array}{l}1: \\
\dddot{11} \\
10\end{array}$ & $\begin{array}{l}29 \\
\ldots \\
25 \\
53\end{array}$ & $\begin{array}{l}10 \\
11 \\
11 \\
10\end{array}$ & $\begin{array}{l}0 \\
7 \\
1 \\
7\end{array}$ & II. & $\begin{array}{l}4 \\
4 \\
4\end{array}$ & $\begin{array}{l}16 \\
00 \\
4 ?\end{array}$ & $\begin{array}{l}11 \\
10 \\
10\end{array}$ & $\begin{array}{l}45 \\
\mathbf{1} 6 \\
27\end{array}$ & $\begin{array}{r}8 \\
10 \\
10\end{array}$ & $\begin{array}{l}5 \\
2 \\
7 \\
0\end{array}$ & II. & $\begin{array}{l}5 \\
5 \\
5\end{array}$ & $\begin{array}{l}101 \\
43 \\
42\end{array}$ & $\begin{array}{l}11 \\
12 \\
10\end{array}$ & $\begin{array}{l}46 \\
33 \\
04\end{array}$ & $\begin{array}{r}? \\
10 \\
111\end{array}$ & $\begin{array}{l}2 \\
5 \\
7\end{array}$ & \multirow{2}{*}{ II. } \\
\hline $\begin{array}{l}3 \\
3 \\
3\end{array}$ & $\begin{array}{l}34 \\
35 \\
0 !\end{array}$ & $\begin{array}{l}10 \\
10 \\
12\end{array}$ & $\begin{array}{l}59 \\
56 \\
09\end{array}$ & $\begin{array}{l}12 \\
10 \\
11\end{array}$ & $\begin{array}{l}4 \\
4 \\
5\end{array}$ & \multirow{6}{*}{$1 \mathrm{II}}$. & $\frac{1}{4}$ & $\begin{array}{l}25 \\
41\end{array}$ & $\begin{array}{l}11 \\
10\end{array}$ & 23 & $\begin{array}{r}9 \\
111\end{array}$ & $\begin{array}{l}6 \\
6\end{array}$ & \multirow{6}{*}{ III. } & $\begin{array}{l}5 \\
5 \\
5\end{array}$ & $\begin{array}{l}09 \\
56 \\
36\end{array}$ & $\begin{array}{l}11 \\
10 \\
12\end{array}$ & $\begin{array}{l}09 \\
53 \\
60\end{array}$ & $\begin{array}{r}10 \\
8 \\
9\end{array}$ & $\begin{array}{l}5 \\
5 \\
7\end{array}$ & \\
\hline 3 & 59 & 10 & 19 & 11 & 0 & & 4 & 47 & 11 & 15 & 9 & 8 & & 5 & 15 & 11 & 18 & 10 & 5 & \multirow{5}{*}{ III. } \\
\hline 3 & 24 & $! 1$ & $0 t 5$ & 10 & 3 & & 4 & 23 & 9 & 07 & 8 & 5 & & 5 & 40 & 10 & 09 & 9 & 0 & \\
\hline 3 & 24 & $\begin{array}{l}12 \\
10\end{array}$ & $\begin{array}{l}13 \\
25\end{array}$ & $\begin{array}{l}12 \\
10\end{array}$ & $\begin{array}{l}3 \\
0\end{array}$ & & 4 & 18 & 12 & $\begin{array}{l}34 \\
34\end{array}$ & 11 & $\frac{6}{7}$ & & $\begin{array}{l}5 \\
5\end{array}$ & 32 & $\because$ & $\dddot{4}$ & $\begin{array}{l}? \\
8\end{array}$ & 6 & \\
\hline $\begin{array}{l}3 \\
3\end{array}$ & $\begin{array}{l}(1111 \\
52\end{array}$ & $\begin{array}{l}10 \\
10\end{array}$ & $\begin{array}{l}25 \\
32\end{array}$ & 9 & 2 & & $\begin{array}{l}4 \\
4\end{array}$ & $\begin{array}{l}52 \\
13\end{array}$ & ... & $\begin{array}{l}34 \\
\ldots\end{array}$ & 110 & ii & & 5 & $\begin{array}{l}10 \\
33\end{array}$ & 10 & $\begin{array}{l}7+ \\
38\end{array}$ & ! & 1 & \\
\hline 3 & 311 & 10 & 39 & 11 & I & & 4 & 56 & 10 & 28 & 9 & 3 & & 5 & 43 & $\mathrm{~J} 1$ & 11 & 10 & 5 & \\
\hline $\begin{array}{l}3 \\
3 \\
3\end{array}$ & $\begin{array}{l}33 \\
32 \\
10\end{array}$ & $\begin{array}{l}\ldots \\
10 \\
11\end{array}$ & $\ldots$ & $\begin{array}{l}10 \\
12 \\
14\end{array}$ & $\begin{array}{l}5 \\
0 \\
0\end{array}$ & IV. & $\begin{array}{l}4 \\
4 \\
4 \\
4\end{array}$ & $\begin{array}{l}22 \\
27 \\
00 \\
51\end{array}$ & $\begin{array}{r}11 \\
10 \\
9 \\
10\end{array}$ & $\begin{array}{l}41 \\
42 \\
13 \\
53\end{array}$ & $\begin{array}{l}10 \\
13 \\
34 \\
14\end{array}$ & $\begin{array}{l}0 \\
0 \\
11 \\
0\end{array}$ & IV. & $\begin{array}{l}5 \\
5\end{array}$ & $\begin{array}{l}11 \\
25\end{array}$ & $\begin{array}{r}10 \\
8\end{array}$ & $\begin{array}{l}23 \\
45\end{array}$ & $\begin{array}{r}9 \\
10\end{array}$ & $\begin{array}{l}0 \\
0\end{array}$ & IV. \\
\hline \multicolumn{21}{|c|}{ MEANS. } \\
\hline $\begin{array}{l}3 \\
3\end{array}$ & $\begin{array}{l}311 \\
2 ! 1\end{array}$ & $\begin{array}{l}10 \\
\ldots\end{array}$ & $\begin{array}{l}57 \\
\ldots\end{array}$ & 10 & $\ldots$ & $\begin{array}{l}19 \\
21\end{array}$ & $\begin{array}{l}4 \\
4\end{array}$ & $\frac{27}{27}$ & $\begin{array}{l}10 \\
\ldots\end{array}$ & $\begin{array}{l}52 \\
\ldots\end{array}$ & $\ldots$ & $\cdots$ & $\begin{array}{l}21 \\
22\end{array}$ & 5 & $\frac{27}{27}$ & $\begin{array}{l}10 \\
\ldots\end{array}$ & $\begin{array}{l}53 \\
\ldots\end{array}$ & $\ldots$ & $\cdots$ & $\begin{array}{l}19 \\
20\end{array}$ \\
\hline $\begin{array}{l}\text { 'I } \\
\text { the }\end{array}$ & $\begin{array}{l}\text { be hi } \\
\text { inter }\end{array}$ & $\begin{array}{l}\text { irglies } \\
\text { val }\end{array}$ & $\begin{array}{l}\text { and } \\
\text { alane }\end{array}$ & lowe & $\begin{array}{l}\text { est ral } \\
\text { arly. }\end{array}$ & lue of & & & & & & & & $\begin{array}{l}\text { T } \\
\text { the } \\
\text { low }\end{array}$ & $\begin{array}{l}\text { he cr } \\
\text { inter } \\
\text { valu }\end{array}$ & $\begin{array}{l}\text { riterio } \\
\text { rval, } \\
\text { nes lo }\end{array}$ & $\begin{array}{l}\text { on rej } \\
\text { the th } \\
\text { alance }\end{array}$ & $\begin{array}{l}\text { fects } \\
\text { wo hi } \\
\text { e near }\end{array}$ & $\begin{array}{l}\text { no } \mathrm{r:} \\
\text { igh at } \\
\text { ily. }\end{array}$ & $\begin{array}{l}\text { lue of } \\
\text { d two }\end{array}$ \\
\hline
\end{tabular}


TABLE For the ReDuction of TIDES. - No. 2.

Showing the Interral between the Apl. Time of the Jloon's Superior Transit and the Time of Iligh Water, and also the Heights of IHigh Water, at Van Rensselaer IIarbor, from Four Series of Ouserrations made between October 10, 1853, and October 22, 1854.

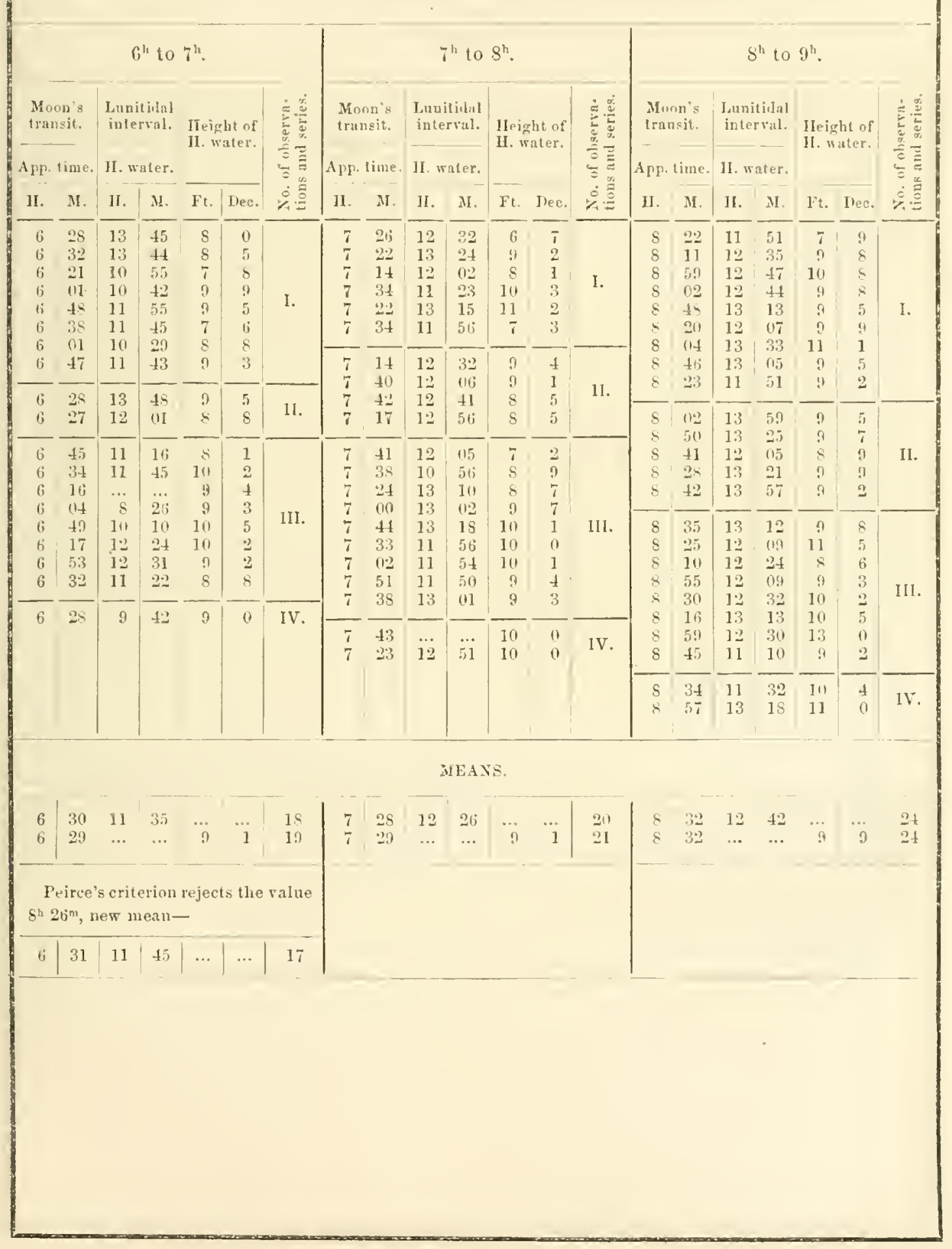


Table for the Rieduction of Tides.-No. 2.

Showing the Interval betwecn the App. Time of the Moon's Superior Transit and the Time of IIjgh Water, and also the Heights of High Water, at Van Ficnsselacr Harbor, from Four Scries of Obserrations made betwcen October 10, 1853, and Octobcr 22, 1854.

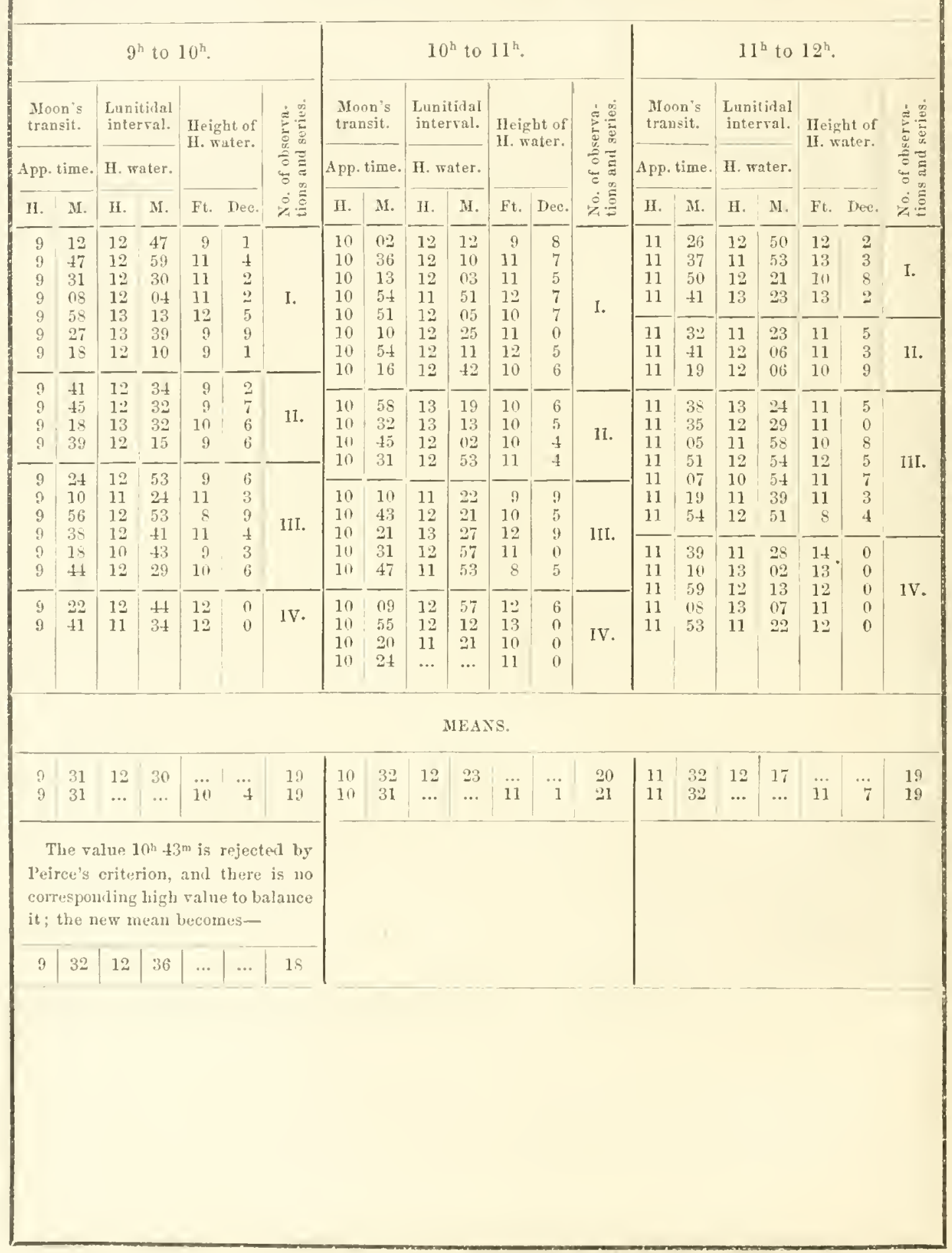


Taber for tie Renuction of T'ides. - No. 2.

Showing the Interval between the $A \mathrm{pl}$. Time of the Moon's Inferior T'ransit and the Time of IIigh Water, and also the IIeights of High Water, at Van Rensselaer IIarbor, from Four Scries of Observations made between Oetober 10, 1853, and October 22, 1854.

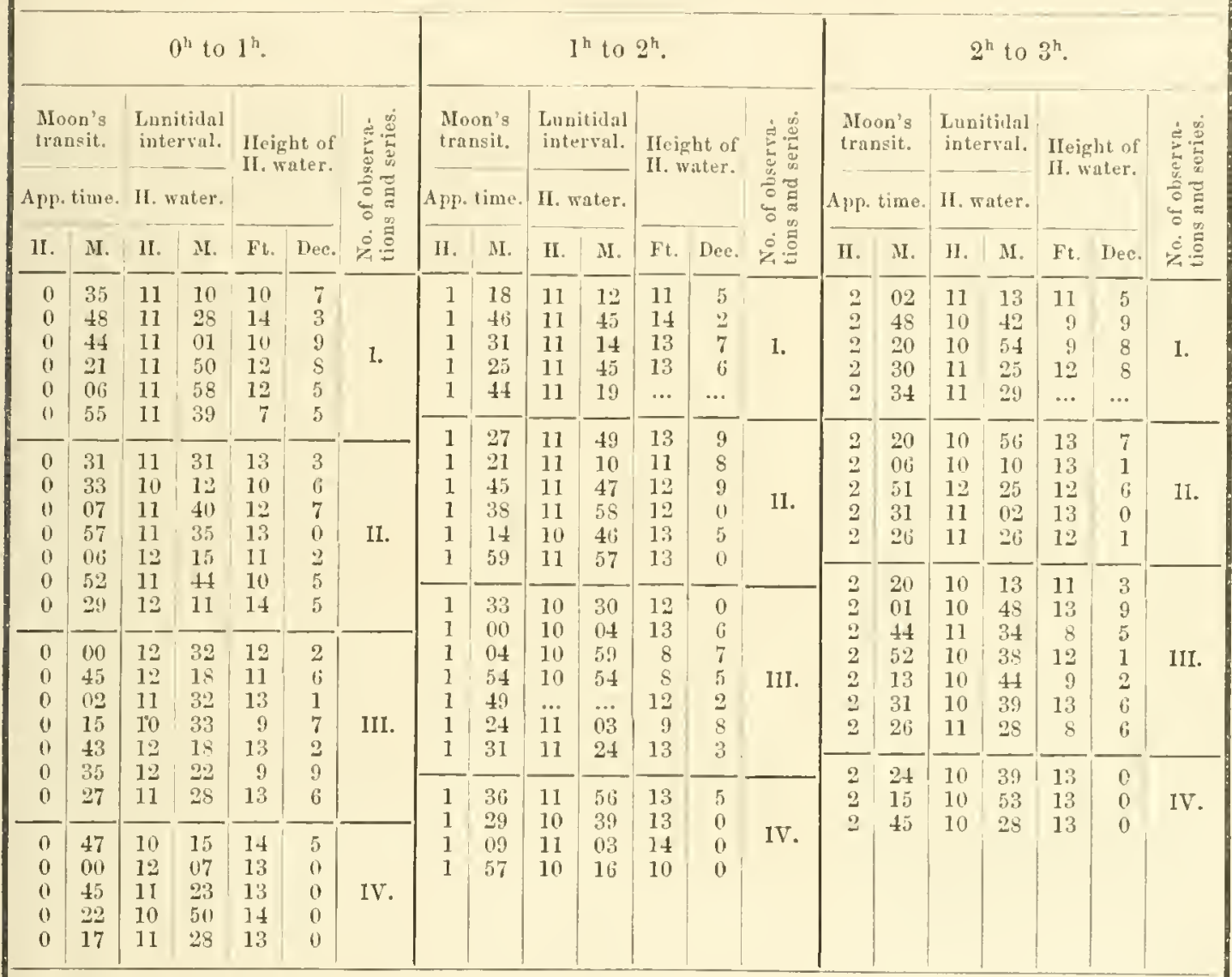

MEANS.

\begin{tabular}{l|l|l|l|l|l|l|l|l|l|l|l|l|l|l|l|l|l|l|l|l|l|l|}
0 & 29 & 11 & 34 & $\ldots$ & $\ldots$ & 25 & 1 & 31 & 11 & 13 & $\ldots$ & $\ldots$ & 21 & 2 & 27 & 10 & 59 & $\ldots$ & $\ldots$ & 20 \\
0 & 29 & $\ldots$ & $\ldots$ & 12 & 2 & 25 & 1 & 31 & $\ldots$ & $\ldots$ & 12 & 3 & 21 & 2 & 27 & $\ldots$ & $\ldots$ & 11 & 8 & 19
\end{tabular}


Table for tue Reduction of Tides.-No. 2.

Showing the Intermal between the Alp. Time of the Moon's Inferior Transit and the Time of High Water, and also the IIeights of IIigh Water, at Van Rensselaer IIarbor, from Four Series of Observations made between October 10, 1853, and Oetober 22, 1854.

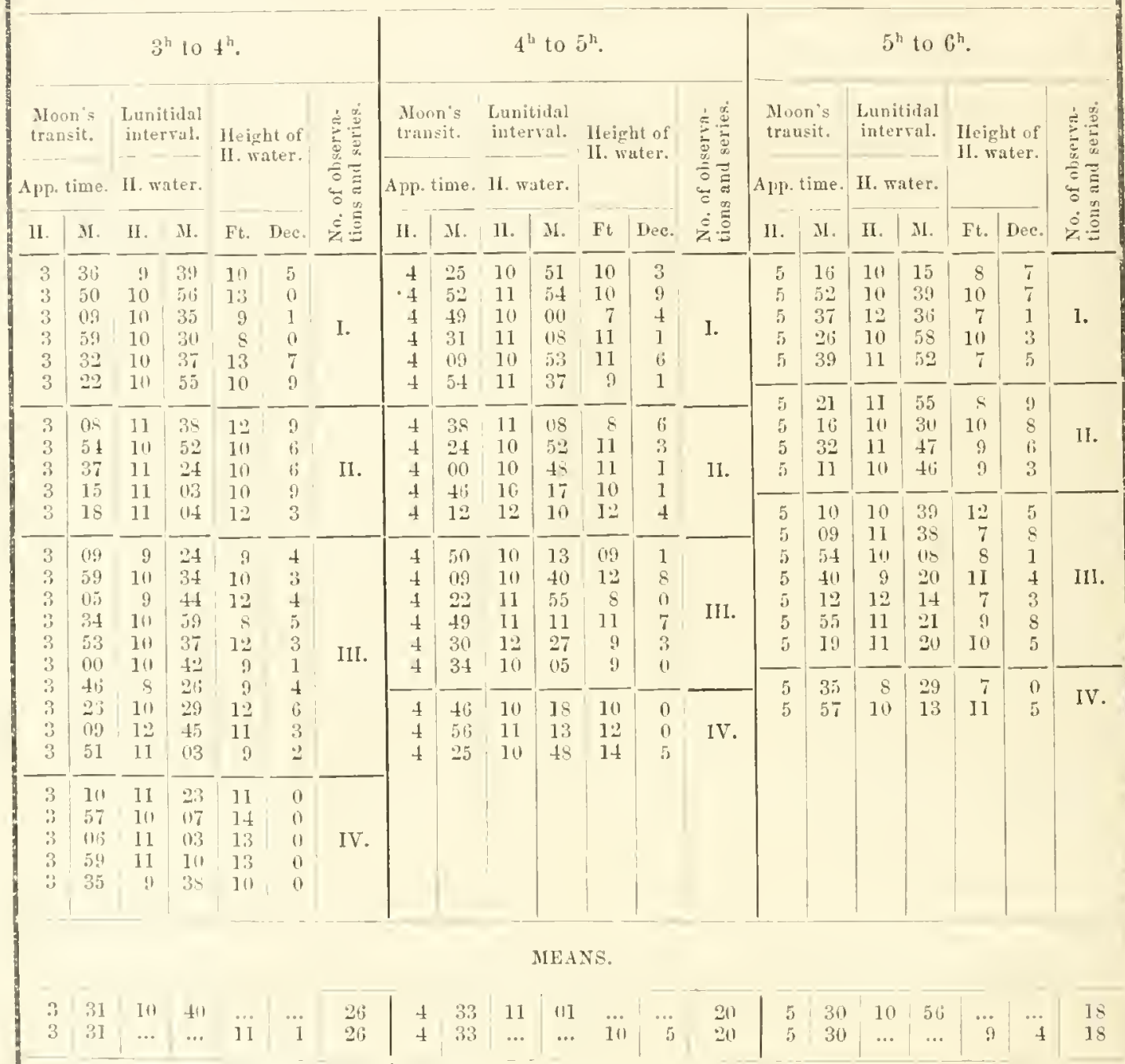

The two greatest deviations from the me.sn, riz., $8^{\text {h }} 26^{\text {m }}$ and $12^{\text {h }} 45^{\mathrm{m}}$, nearly labanco in the mean, hence no rallue was rejertitid.

The criterion rejects no value of the interval; the low ralue sh 29 m is so near thr limit of rejection and not balancol in the mean that prefure to reject it.

\begin{tabular}{l|l|l|l|l|l|l}
5 & 30 & 11 & 114 & $\ldots$ & $\ldots$ & 17
\end{tabular} 


\section{'TABLE for the lieduetion of Tides. - No. 2.}

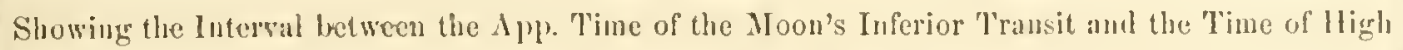
Water, and also the Ileights of High Water, at Van Rensselacr Ilarbor, from Four Series of Observations made between October 10, 1853, and Oetober 22, 1854 .

\begin{tabular}{|c|c|c|c|c|c|c|c|c|c|c|c|c|c|c|c|c|c|c|c|c|}
\hline & & & & & & & & & & ${ }^{h}$ to 8 & $8^{\text {h. }}$ & & & & & & to 5 & $9^{\mathrm{h}}$. & & \\
\hline $\begin{array}{l}\text { Mloo } \\
\text { tran }\end{array}$ & $\begin{array}{l}\text { n's } \\
\text { sit. }\end{array}$ & $\begin{array}{l}\text { Lunit } \\
\text { inter }\end{array}$ & $\begin{array}{l}\text { tidal } \\
\text { val. }\end{array}$ & $\begin{array}{l}\text { Ileigh } \\
\text { II. wat }\end{array}$ & $\begin{array}{l}\text { hat of } \\
\text { ater. }\end{array}$ & 离部 & $\begin{array}{l}M 100 \\
\text { tran }\end{array}$ & $\begin{array}{l}\text { n's } \\
\text { sit. }\end{array}$ & $\begin{array}{l}\text { Lunit } \\
\text { inter }\end{array}$ & $\begin{array}{l}\text { ticlal } \\
\text { rval. }\end{array}$ & $\begin{array}{l}\text { Ireigh } \\
\text { II. wa }\end{array}$ & & 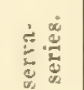 & $\begin{array}{l}\text { M10o } \\
\text { tran }\end{array}$ & $\begin{array}{l}\text { n's } \\
\text { it. }\end{array}$ & $\begin{array}{l}\text { Iunit } \\
\text { inter }\end{array}$ & & $\begin{array}{l}\text { Ifeigh } \\
\text { If } w a\end{array}$ & & 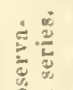 \\
\hline A pp. & time. & II. พล & nter. & & & 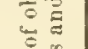 & $\Lambda \mathrm{pp}$. & time. & II. IF & ater. & & & & App. & time. & II. wa & ter. & & & \\
\hline 11. & M. & 11. & M. & Ft. & Dee. & 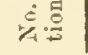 & II. & M. & II. & M. & Ft. & Dec. & $\dot{8}$ & II. & II. & I1. & II. & Ft. I & Dec. & $\dot{8}$ \\
\hline $\begin{array}{l}6 \\
6 \\
6 \\
6 \\
6 \\
6 \\
6 \\
6\end{array}$ & $\begin{array}{l}57 \\
17 \\
57 \\
48 \\
25 \\
14 \\
24\end{array}$ & $\begin{array}{l}11 \\
10 \\
12 \\
11 \\
13 \\
13 \\
12\end{array}$ & $\begin{array}{l}16 \\
54 \\
49 \\
58 \\
48 \\
39 \\
36\end{array}$ & $\begin{array}{r}9 \\
7 \\
7 \\
10 \\
8 \\
10 \\
7\end{array}$ & $\begin{array}{l}2 \\
1 \\
0 \\
4 \\
5 \\
6 \\
8\end{array}$ & 1. & \begin{tabular}{l|l}
7 & \\
7 & \\
7 & \\
7 & \\
7 & \\
7 &
\end{tabular} & $\begin{array}{l}54 \\
46 \\
38 \\
11 \\
56 \\
42 \\
10\end{array}$ & $\begin{array}{l}11 \\
12 \\
11 \\
12 \\
12 \\
12 \\
12\end{array}$ & $\begin{array}{l}49 \\
30 \\
53 \\
37 \\
46 \\
25 \\
50\end{array}$ & $\begin{array}{r}9 \\
8 \\
11 \\
8 \\
9 \\
11 \\
8\end{array}$ & $\begin{array}{l}5 \\
1 \\
0 \\
8 \\
4 \\
8 \\
0\end{array}$ & 1. & $\begin{array}{l}8 \\
8 \\
8 \\
8 \\
8 \\
8\end{array}$ & $\begin{array}{l}47 \\
35 \\
25 \\
41 \\
25 \\
50\end{array}$ & $\begin{array}{l}12 \\
\ldots \\
13 \\
12 \\
12 \\
12\end{array}$ & $\begin{array}{l}42 \\
\ldots \\
21 \\
5 n \\
42 \\
38\end{array}$ & \begin{tabular}{r|}
10 \\
9 \\
10 \\
10 \\
9 \\
11
\end{tabular} & $\begin{array}{l}0 \\
3 \\
7 \\
9 \\
2 \\
2\end{array}$ & I. \\
\hline & & & & & & & 7 & 59 & 12 & 30 & 11 & 5 & & 8 & 26 & 12 & 49 & ${ }^{6}$ & $i$ & \\
\hline $\begin{array}{l}6 \\
6 \\
6\end{array}$ & 51 & $\begin{array}{l}11 \\
13 \\
11\end{array}$ & $\begin{array}{l}10 \\
41\end{array}$ & $\begin{array}{r}71 \\
6\end{array}$ & $\begin{array}{l}8 \\
2 \\
9\end{array}$ & 11. & $\begin{array}{l}7 \\
7 \\
7\end{array}$ & $\begin{array}{l}35 \\
\text { II }\end{array}$ & $\begin{array}{l}11 \\
12\end{array}$ & $\begin{array}{l}53 \\
12 \\
17\end{array}$ & $\begin{array}{l}8 \\
9 \\
-\end{array}$ & $\frac{2}{5}$ & 1t. & $\begin{array}{l}8 \\
8 \\
8 \\
\end{array}$ & $\begin{array}{l}10 \\
53 \\
12\end{array}$ & $\begin{array}{l}17 \\
13 \\
11\end{array}$ & $\begin{array}{l}57 \\
42\end{array}$ & $\begin{array}{r}10 \\
810\end{array}$ & $\begin{array}{l}8 \\
8 \\
2\end{array}$ & 11. \\
\hline 6 & 52 & & & & 7 & & 7 & 41 & & & 7 & & & 8 & 07 & 11 & 55 & 10 & 4 & \\
\hline $\begin{array}{l}6 \\
6 \\
6 \\
6 \\
6 \\
6 \\
6\end{array}$ & $\begin{array}{l}07 \\
59 \\
38 \\
27 \\
39 \\
07 \\
59\end{array}$ & $\begin{array}{l}11 \\
12 \\
12 \\
10 \\
12 \\
11 \\
13\end{array}$ & $\begin{array}{l}12 \\
20 \\
54 \\
32 \\
17 \\
47 \\
25\end{array}$ & $\begin{array}{r}11 \\
9 \\
8 \\
10 \\
9 \\
9 \\
9\end{array}$ & \begin{tabular}{l|}
7 \\
9 \\
6 \\
2 \\
8 \\
8 \\
5 \\
4
\end{tabular} & III. & $\begin{array}{l}7 \\
7 \\
7 \\
7 \\
7 \\
7 \\
7\end{array}$ & $\begin{array}{l}13 \\
47 \\
22 \\
11 \\
54 \\
26 \\
16\end{array}$ & $\begin{array}{l}12 \\
11 \\
13 \\
11 \\
12 \\
12 \\
13\end{array}$ & $\begin{array}{l}14 \\
47 \\
411 \\
48 \\
05 \\
30 \\
38\end{array}$ & $\begin{array}{r}10 \\
11 \\
9 \\
10 \\
9 \\
10 \\
7\end{array}$ & $\begin{array}{l}0 \\
1 \\
2 \\
5 \\
9 \\
6 \\
6\end{array}$ & 111. & $\begin{array}{l}8 \\
8 \\
8 \\
8 \\
8 \\
8 \\
8 \\
8\end{array}$ & $\begin{array}{l}59 \\
112 \\
48 \\
32 \\
07 \\
54 \\
37 \\
1\end{array}$ & $\begin{array}{l}11 \\
1: 3 \\
13 \\
11 \\
11 \\
12 \\
12 \\
12\end{array}$ & $\begin{array}{l}18 \\
32 \\
31 \\
32 \\
55 \\
09 \\
22 \\
38\end{array}$ & $\begin{array}{r}10 \\
7 \\
8 \\
10 \\
10 \\
11 \\
9 \\
11\end{array}$ & $\begin{array}{l}7 \\
9 \\
1 \\
6 \\
4 \\
1 \\
4 \\
2\end{array}$ & 111. \\
\hline $\begin{array}{l}6 \\
6\end{array}$ & 59 & 10 & 41 & 10 & 0 & 1V. & $\cdots$ & $\ldots$ & $\ldots$ & $\ldots$ & $\ldots$ & $\ldots$ & IV. & $\mathrm{s}$ & 01 & 13 & 53 & 8 & $\tilde{7}$ & \\
\hline & & & & & & & & & & & & & & $\begin{array}{l}8 \\
8 \\
8\end{array}$ & $\begin{array}{l}08 \\
58 \\
34\end{array}$ & $\begin{array}{l}11 \\
\ldots \\
13\end{array}$ & $\begin{array}{l}58 \\
\ldots 1 \\
-4\end{array}$ & $\begin{array}{r}9 \\
9 \\
10\end{array}$ & $\begin{array}{l}1 \\
0 \\
0\end{array}$ & 1V. \\
\hline & & & & & & & & & & IEAN & & & & & & & & & & \\
\hline $\begin{array}{l}6 \\
6\end{array}$ & $\begin{array}{l}33 \\
34\end{array}$ & $\begin{array}{l}12 \\
\cdots\end{array}$ & $\begin{array}{l}13 \\
\cdots\end{array}$ & .. & $\ddot{\mathrm{i}}$ & $\begin{array}{l}19 \\
20\end{array}$ & 7 & $\begin{array}{l}33 \\
33\end{array}$ & $\begin{array}{l}12 \\
\ldots\end{array}$ & \begin{tabular}{l|l}
25 & \\
$\cdots$ &
\end{tabular} & $\ddot{9}$ & $\ddot{6}$ & $\begin{array}{l}18 \\
18\end{array}$ & $\begin{array}{l}8 \\
8\end{array}$ & $\begin{array}{l}29 \\
30\end{array}$ & $\begin{array}{l}12 \\
\ldots\end{array}$ & $\begin{array}{l}44 \\
\cdots\end{array}$ & ... & $\cdots$ & $\begin{array}{l}21 \\
23\end{array}$ \\
\hline $\begin{array}{l}\mathrm{T} \\
\text { valu } \\
10^{\mathrm{h}} \\
\text { eacl } \\
\text { reje }\end{array}$ & $\begin{array}{l}\text { 'here } \\
\text { ues, } \\
32 \text {, } \\
\text { h oth } \\
\text { cetion }\end{array}$ & $\begin{array}{c}\text { are } \\
\text { viz., } \\
1 \text { (th } \\
\text { r; th } \\
\text { requ }\end{array}$ & $\begin{array}{l}\text { wo } \mathrm{h} \\
4^{\text {h }} 06 \\
11^{\mathrm{m}}, \mathrm{t} \\
\text { tere } \mathrm{w}\end{array}$ & $\begin{array}{l}\text { ight at } \\
\text { f'm, } 13 \\
\text { uearly } \\
\text { vas, th }\end{array}$ & $\begin{array}{l}\text { uol } t \pi \\
48^{\text {tw }} \\
\text { hala }\end{array}$ & $\begin{array}{l}\text { tow } \\
\text { o and } \\
\text { incing } \\
\text { re, no }\end{array}$ & $\begin{array}{r}\mathrm{T} \\
\text { inte }\end{array}$ & $\begin{array}{l}\text { he his } \\
\text { rval b }\end{array}$ & $\begin{array}{l}\text { igh at } \\
\text { balau }\end{array}$ & $\begin{array}{l}\text { nd lo } \\
\text { cee. }\end{array}$ & ow val & lues in & the & & & & & & & \\
\hline
\end{tabular}




\section{TABle for the Redection of TIdes. - Nó. 2.}

Showing the Interval betreen the App. Time of the MLon's Inferior Transit and the Time of High

Wrater, and also the Heights of High Water, at Van Rensselaer IIarbor, from Four Series of

Observations made between October 10, 1853, and October 22, 1854 .

\begin{tabular}{|c|c|c|c|c|c|c|c|c|c|c|c|c|c|c|c|c|c|c|c|c|}
\hline \multicolumn{7}{|c|}{$9^{\mathrm{h}}$ to $10^{\mathrm{h}}$. } & \multicolumn{7}{|c|}{$10^{\mathrm{h}}$ to $11^{\mathrm{h}}$. } & \multicolumn{7}{|c|}{$11^{\mathrm{h}}$ to $12^{\mathrm{h}}$. } \\
\hline \multicolumn{2}{|c|}{$\begin{array}{l}\text { Moon's } \\
\text { transit. }\end{array}$} & \multirow{2}{*}{\multicolumn{2}{|c|}{$\begin{array}{c}\text { Lunitidal } \\
\text { interval. } \\
\text { II. Tater. }\end{array}$}} & \multirow{2}{*}{\multicolumn{2}{|c|}{$\begin{array}{l}\text { Height of } \\
\text { II. water. }\end{array}$}} & \multirow{3}{*}{ 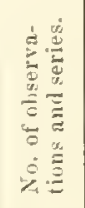 } & \multirow{2}{*}{\multicolumn{2}{|c|}{$\begin{array}{c}\begin{array}{c}\text { Moon's } \\
\text { transit. }\end{array} \\
\text { App.time. }\end{array}$}} & \multirow{2}{*}{\multicolumn{2}{|c|}{$\begin{array}{l}\begin{array}{l}\text { Lunitidal } \\
\text { interval. }\end{array} \\
\text { ПI. water. }\end{array}$}} & \multirow{2}{*}{\multicolumn{2}{|c|}{$\begin{array}{l}\text { Meight of } \\
\text { 11. water. }\end{array}$}} & \multirow{3}{*}{ 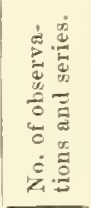 } & \multirow{2}{*}{\multicolumn{2}{|c|}{$\frac{\begin{array}{c}\text { Moon's } \\
\text { transit. }\end{array}}{\text { A pr. time. }}$}} & \multirow{2}{*}{\multicolumn{2}{|c|}{$\begin{array}{l}\text { Lunitidal } \\
\text { interval. } \\
\text { H. water. }\end{array}$}} & \multirow{2}{*}{\multicolumn{2}{|c|}{$\begin{array}{l}\text { IIeight of } \\
\text { II. water. }\end{array}$}} & \multirow{3}{*}{ 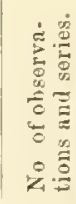 } \\
\hline \multicolumn{2}{|c|}{ Alp. tirne. } & & & & & & & & & & & & & & & & & & & \\
\hline H. & 31. & II. & $\mathrm{M}$. & Ft. & Dee. & & 11. & M. & II. & M. & $\mathrm{Ft}$. & Dec. & & 11. & M. & II. & M. & Ft. & Dec. & \\
\hline 9 & 37 & 12 & 37 & 10 & 9 & \multirow{7}{*}{ I. } & 10 & 24 & 12 & 20 & 11 & 3 & \multirow{5}{*}{ I. } & 11 & 01 & 12 & 30 & 12 & 0 & \multirow{6}{*}{ I. } \\
\hline 9 & 23 & 12 & 23 & 10 & 7 & & 10 & 11 & 13 & 50 & 11 & 6 & & 11 & 52 & 11 & 54 & 12 & 3 & \\
\hline 9 & 09 & & 22 & 11 & 8 & & 10 & 34 & 12 & 41 & 11 & 0 & & 11 & 15 & 11 & 30 & 3 & 8 & \\
\hline 9 & 52 & 11 & 54 & 11 & 5 & & 10 & 24 & 13 & 17 & 13 & 8 & & \multirow{3}{*}{11} & 59 & 12 & 01 & 13 & 0 & \\
\hline 9 & 33 & 12 & 39 & 12 & 0 & & 10 & 31 & 11 & 49 & 8 & 6 & & & \multirow{2}{*}{$\begin{array}{l}20 \\
17\end{array}$} & \multirow{2}{*}{$\begin{array}{l}11 \\
11\end{array}$} & 36 & 13 & $i$ & \\
\hline 9 & 06 & 12 & 30 & 7 & 8 & & 10 & 06 & 13 & 39 & $f$ & 6 & & & & & 45 & 8 & 9 & \\
\hline 9 & $\begin{array}{l}49 \\
46\end{array}$ & 12 & 27 & 11 & 6 & & 10 & 57 & 12 & 48 & 7 & 7 & & 11 & 30 & 12 & 47 & 12 & 4 & \\
\hline & & & & & & & 10 & 15 & 12 & 02 & 11 & 2 & 1I. & 11 & 46 & 11 & 29 & 8 & 4 & II. \\
\hline $\begin{array}{l}9 \\
9\end{array}$ & $\begin{array}{l}15 \\
12\end{array}$ & $\begin{array}{l}12 \\
12\end{array}$ & $\begin{array}{l}3 ! 1 \\
05\end{array}$ & $\begin{array}{r}7 \\
10\end{array}$ & $\stackrel{2}{3}$ & II. & $\begin{array}{l}10 \\
10\end{array}$ & 55 & 11 & 59 & 11 & 9 & & 11 & 43 & 11 & $\begin{array}{l}34 \\
42\end{array}$ & 12 & 0 & \\
\hline 9 & 11 & 12 & 43 & 111 & 3 & & 10 & 32 & & 30 & 12 & 2 & & 11 & 16 & 12 & 46 & 12 & 0 & \\
\hline 9 & 47 & 11 & 15 & 11 & 1 & & 10 & 19 & 13 & 45 & 11 & 8 & & 11 & 09 & 11 & 40 & 13 & 7 & \\
\hline 9 & 33 & 11 & 31 & 9 & 4 & & 10 & 43 & 11 & 35 & 10 & 0 & 111 & 11 & 28 & 13 & 17 & 9 & 7 & 111 \\
\hline 9 & 16 & 10 & 45 & 11 & 3 & III. & 10 & 35 & 10 & 23 & 12 & 9 & III. & 11 & 35 & 13 & 53 & 11 & 4 & III. \\
\hline 9 & 59 & 10 & 49 & 9 & 9 & 111. & 10 & 07 & 11 & 21 & 8 & 5 & & 11 & 44 & 12 & 14 & 8 & 3 & \\
\hline 9 & 41 & 12 & 17 & 11 & is & & 10 & 55 & 13 & 18 & s & 1 & & 11 & 20 & 12 & 20 & 12 & 5 & \\
\hline 9 & 22 & 12 & 06 & & 9 & & 10 & 45 & 12 & $2 i j$ & 14 & 0 & & 11 & 17 & 11 & 50 & 11 & 5 & \\
\hline 9 & $4 i$ & 111 & 49 & 10 & 0 & $1 \mathrm{Y}$ & 10 & 02 & 11 & 13 & 12 & 0 & IV. & 11 & 36 & 12 & 96 & 14 & 0 & IV. \\
\hline 9 & 19 & 11 & 56 & 11 & 0 & 18. & 10 & 46 & 12 & 29 & 12 & 0 & & 11 & 30 & 12 & 15 & 12 & 0 & \\
\hline & & & & & & & & & & IEAN & & & & & & & & & & \\
\hline 9 & 30 & 12 & 03 & & & 19 & 10 & 29 & 12 & 18 & &.. & 19 & 11 & 28 & 12 & 16 & $\because$ & $\ldots$ & 19 \\
\hline 9 & 30 & $\ldots$ & $\ldots$ & 10 & 4 & 19 & 10 & 29 & $\ldots$ & $\ldots$ & 10 & 9 & 19 & 11 & 25 & $\ldots$ & $\ldots$ & 11 & 5 & 19 \\
\hline $\begin{array}{l}1 \\
\text { one } \\
\text { sies } \\
\text { res } \\
4 y^{\prime \prime}\end{array}$ & $\begin{array}{l}\text { here } \\
\text { ligh } \\
\text { ned I } \\
\text { Iting } \\
\text { viz: }\end{array}$ & $\begin{array}{c}\text { bein } \\
\text { valt } \\
\text { refar } \\
\text { afte } \\
-\end{array}$ & $\begin{array}{l}8 \text { ther } \\
\text { de in } \\
\text { able } \\
\text { o the }\end{array}$ & $\begin{array}{l}\text { ee lo } \\
\text { the } \\
\text { to ad } \\
\text { rejee }\end{array}$ & $\begin{array}{l}\text { ow an } \\
\text { inters } \\
\text { lopt a } \\
\text { etion }\end{array}$ & $\begin{array}{l}\text { d hut } \\
\text { val, it } \\
\text { mean } \\
\text { of } 10^{\text {h }}\end{array}$ & & & & & & & & & & & & & & \\
\hline 9 & 30 & 12 & 07 & $\ldots$ & $\ldots$ & 18 & & & & & & & & & & & & & & \\
\hline & & & & & & & & & & & & & & & & & & & & \\
\hline & & & & & & & & & & & & & & & & & & & & \\
\hline & & & & & & & & & & & & & & & & & & & & \\
\hline & & & & & & & & & & & & & & & & & & & & \\
\hline & & & & & & & & & & & & & & & & & & & & \\
\hline & & & & & & & & & & & & & & & & & & & & \\
\hline & & & & & & & & & & & & & & & & & & & & \\
\hline
\end{tabular}


TABLe mor the Reduction of 'THES.-Xo. 2.

Showing the Juterval between the $A$ ply. Time of the Moon's Superior Transit and the 'Time of Low Watex, and also the Ideights of Low Water, at Tan Rensselaer Ilarbor, from Four Series of Observations made between Oetober 10, 1853, and Oetober 22, 1854.

\begin{tabular}{|c|c|c|c|c|c|c|c|c|c|c|c|c|c|c|c|c|c|c|c|c|}
\hline \multicolumn{7}{|c|}{$0^{\mathrm{h}}$ to $1^{\mathrm{h}}$. } & \multicolumn{7}{|c|}{$1^{\mathrm{h}}$ to $2^{\mathrm{h}}$. } & \multicolumn{7}{|c|}{$2^{h}$ to $3^{h}$. } \\
\hline \multicolumn{2}{|c|}{$\begin{array}{l}\text { Moon's } \\
\text { transit. }\end{array}$} & \multirow{2}{*}{\multicolumn{2}{|c|}{$\begin{array}{c}\begin{array}{c}\text { Lunitilal } \\
\text { interval. }\end{array} \\
\text { L. water. }\end{array}$}} & \multirow{2}{*}{\multicolumn{2}{|c|}{$\begin{array}{l}\text { 1leight of } \\
\text { L. wuter. }\end{array}$}} & \multirow{3}{*}{ 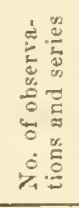 } & \multirow{2}{*}{\multicolumn{2}{|c|}{$\begin{array}{c}\begin{array}{c}\text { Moon's } \\
\text { transit. }\end{array} \\
\Delta_{\text {[P. time. }}\end{array}$}} & \multirow{2}{*}{\multicolumn{2}{|c|}{$\frac{\begin{array}{c}\text { Lunitidal } \\
\text { interval. }\end{array}}{\text { L. water. }}$}} & \multirow{2}{*}{\multicolumn{2}{|c|}{$\begin{array}{l}\text { Hoight of } \\
\text { I. witer. }\end{array}$}} & \multirow{3}{*}{ 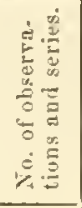 } & \multirow{2}{*}{\multicolumn{2}{|c|}{$\begin{array}{c}\text { Moon's } \\
\text { transit. } \\
\text { Alp.time. }\end{array}$}} & \multirow{2}{*}{\multicolumn{2}{|c|}{$\begin{array}{c}\text { Linitidal } \\
\text { interval. }\end{array}$}} & \multirow{2}{*}{\multicolumn{2}{|c|}{$\begin{array}{l}\text { II eight of } \\
\text { L. water. }\end{array}$}} & \multirow{3}{*}{ 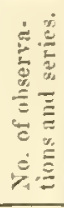 } \\
\hline \multicolumn{2}{|c|}{ App. time. } & & & & & & & & & & & & & & & & & & & \\
\hline II. & M. & II & $\mathrm{M}$. & Ft. & Dec. & & I1. & $\mathrm{H}$. & 11. & M. & Ft. & Dee. & & II. & II. & 1I. & M. & Ft. & Dee. & \\
\hline $\begin{array}{l}0 \\
0 \\
0 \\
0 \\
0\end{array}$ & $\begin{array}{l}13 \\
57 \\
19 \\
21 \\
53\end{array}$ & $\begin{array}{l}17 \\
17 \\
18 \\
18 \\
17\end{array}$ & $\begin{array}{l}02 \\
33 \\
42 \\
54 \\
17\end{array}$ & $\begin{array}{r}1 \\
2 \\
6 \\
3 \\
-2\end{array}$ & $\begin{array}{l}5 \\
0 \\
5 \\
1 \\
1\end{array}$ & \multirow[t]{2}{*}{ I. } & $\begin{array}{l}1 \\
1 \\
1 \\
1 \\
1\end{array}$ & $\begin{array}{l}40 \\
16 \\
07 \\
55 \\
58\end{array}$ & $\begin{array}{l}17 \\
18 \\
17 \\
18 \\
17\end{array}$ & $\begin{array}{l}35 \\
00 \\
38 \\
20 \\
12\end{array}$ & $\begin{array}{r}1 \\
0 \\
3 \\
1 \\
-0\end{array}$ & $\begin{array}{l}9 \\
0 \\
1 \\
2 \\
4\end{array}$ & \multirow[t]{2}{*}{1.} & $\begin{array}{l}2 \\
2 \\
2 \\
2 \\
\end{array}$ & $\begin{array}{l}25 \\
16 \\
44 \\
58\end{array}$ & $\begin{array}{l}18 \\
17 \\
17 \\
17\end{array}$ & $\begin{array}{l}35 \\
45 \\
311 \\
114\end{array}$ & $\begin{array}{r}2 \\
0 \\
2 \\
\cdots \\
\end{array}$ & $\begin{array}{c}7 \\
3 \\
3 \\
\cdots \\
\end{array}$ & I. \\
\hline 0 & 30 & 18 & 49 & 1 & 6 & & 1 & 20 & 15 & 58 & 0 & 6 & & \multirow{2}{*}{$\begin{array}{l}2 \\
2 \\
2 \\
2 \\
2 \\
2 \\
2 \\
\end{array}$} & $\begin{array}{l}45 \\
29\end{array}$ & 18 & $0 \mathrm{I}$ & $\begin{array}{l}1 \\
2\end{array}$ & $\begin{array}{l}3 \\
3\end{array}$ & \multirow[b]{2}{*}{ Il. } \\
\hline $\begin{array}{l}0 \\
0 \\
0 \\
0 \\
0\end{array}$ & $\begin{array}{l}111 \\
111 \\
58 \\
33 \\
29\end{array}$ & $\begin{array}{l}18 \\
17 \\
18 \\
17 \\
19\end{array}$ & $\begin{array}{l}16 \\
05 \\
18 \\
44 \\
37\end{array}$ & $\begin{array}{l}1 \\
4 \\
2 \\
0 \\
2\end{array}$ & $\begin{array}{l}3 \\
1 \\
6 \\
1 \\
7\end{array}$ & \multirow[t]{3}{*}{11.} & $\begin{array}{l}1 \\
1 \\
1 \\
1 \\
1\end{array}$ & $\begin{array}{l}01 \\
5.5 \\
44 \\
2.2 \\
15\end{array}$ & $\begin{array}{l}17 \\
17 \\
18 \\
17 \\
17\end{array}$ & $\begin{array}{l}00 \\
21 \\
02 \\
40 \\
21\end{array}$ & $\begin{array}{r}0 \\
-0 \\
4 \\
0 \\
3\end{array}$ & $\begin{array}{l}5 \\
3 \\
1 \\
5 \\
0\end{array}$ & \multirow[t]{2}{*}{ II. } & & $\begin{array}{l}119 \\
53 \\
02 \\
52\end{array}$ & $\begin{array}{l}17 \\
17 \\
18 \\
17 \\
\end{array}$ & $\begin{array}{l}39 \\
25 \\
19 \\
15\end{array}$ & $\begin{array}{l}2 \\
2 \\
1 \\
3\end{array}$ & $\begin{array}{l}5 \\
5 \\
2 \\
1 \\
\end{array}$ & \\
\hline 0 & 07 & 18 & is & $\begin{array}{l}\tilde{3} \\
3\end{array}$ & $\begin{array}{l}8 \\
5 \\
3\end{array}$ & & 1 & 36 & 38 & 20 & 1 & 5 & & \multirow{3}{*}{$\begin{array}{l}2 \\
2 \\
2 \\
2 \\
2 \\
2 \\
2 \\
2\end{array}$} & $\begin{array}{l}45 \\
33\end{array}$ & 18 & 18 & 3 & 9 & \multirow[b]{3}{*}{ III. } \\
\hline 0 & & & & & & & 1 & 09 & 18 & 24 & 2 & 1 & \multirow{3}{*}{ III. } & & $\begin{array}{l}33 \\
1.9\end{array}$ & 17 & $\begin{array}{l}46 \\
59\end{array}$ & $\begin{array}{l}1 \\
2\end{array}$ & $\begin{array}{l}2 \\
5 \\
5\end{array}$ & \\
\hline $\begin{array}{l}0 \\
0 \\
0 \\
0 \\
0\end{array}$ & $\begin{array}{l}22 \\
30 \\
39 \\
10 \\
111\end{array}$ & $\begin{array}{l}16 \\
16 \\
16 \\
15 \\
16\end{array}$ & $\begin{array}{l}41 \\
49 \\
09 \\
21 \\
45\end{array}$ & $\begin{array}{l}2 \\
1 \\
1 \\
2 \\
2\end{array}$ & $\begin{array}{l}0 \\
3 \\
6 \\
0 \\
0\end{array}$ & \multirow[t]{2}{*}{ III. } & $\begin{array}{l}1 \\
1 \\
1 \\
1 \\
1\end{array}$ & $\begin{array}{l}56 \\
30 \\
29 \\
119 \\
49\end{array}$ & $\begin{array}{l}15 \\
16 \\
17 \\
17 \\
18\end{array}$ & $\begin{array}{l}37 \\
49 \\
34 \\
15 \\
08\end{array}$ & $\begin{array}{l}1 \\
0 \\
1 \\
0 \\
3\end{array}$ & $\begin{array}{l}9 \\
8 \\
5 \\
4 \\
2\end{array}$ & & & $\begin{array}{l}21 \\
37 \\
02 \\
45\end{array}$ & $\begin{array}{l}17 \\
17 \\
17 \\
17\end{array}$ & $\begin{array}{l}39 \\
20 \\
18 \\
21\end{array}$ & $\begin{array}{l}1 \\
3 \\
1 \\
1\end{array}$ & $\begin{array}{l}1 \\
6 \\
2 \\
7\end{array}$ & \\
\hline 0 & 59 & 15 & 58 & 1 & 9 & & I & 00 & 17 & 10 & 1 & 2 & & \multirow{2}{*}{$\begin{array}{l}2 \\
2 \\
3 \\
2 \\
2\end{array}$} & \multirow{2}{*}{$\begin{array}{l}01 \\
47 \\
39 \\
21\end{array}$} & \multirow{2}{*}{$\begin{array}{l}17 \\
19 \\
16 \\
17\end{array}$} & \multirow{2}{*}{$\begin{array}{l}31 \\
16 \\
30 \\
52\end{array}$} & -11 & $\begin{array}{l}5 \\
10\end{array}$ & \\
\hline $\begin{array}{l}0 \\
0\end{array}$ & 22 & $\begin{array}{l}17 \\
16\end{array}$ & $\begin{array}{l}46 \\
56\end{array}$ & $\begin{array}{r}-0 \\
0\end{array}$ & $\begin{array}{l}7 \\
0\end{array}$ & 1V. & $\begin{array}{l}1 \\
1 \\
1 \\
1\end{array}$ & $\begin{array}{l}12 \\
(17 \\
52 \\
33\end{array}$ & $\begin{array}{l}18 \\
18 \\
18 \\
17\end{array}$ & $\begin{array}{l}50 \\
01 \\
]_{6} \\
40\end{array}$ & $\begin{array}{l}0 \\
2 \\
0 \\
0\end{array}$ & $\begin{array}{l}0 \\
0 \\
0 \\
0\end{array}$ & IV. & & & & & $\begin{array}{l}1 \\
0\end{array}$ & i & IV. \\
\hline & & & & & & & & & & $1 \mathrm{EAN}$ & & & & & & & & & & \\
\hline $\begin{array}{l}0 \\
0\end{array}$ & $\begin{array}{l}30 \\
30\end{array}$ & $\begin{array}{l}17 \\
\cdots\end{array}$ & $\begin{array}{l}49 \\
\cdots\end{array}$ & $\ddot{1}$ & $\dddot{7}$ & $\begin{array}{l}21 \\
21\end{array}$ & $\begin{array}{l}1 \\
1\end{array}$ & $\begin{array}{l}29 \\
29\end{array}$ & $\begin{array}{l}17 \\
\cdots\end{array}$ & $\begin{array}{l}34 \\
\cdots\end{array}$ & $\dddot{1}$ & $\ddot{3}$ & $\begin{array}{l}23 \\
23\end{array}$ & $\begin{array}{l}2 \\
2\end{array}$ & $\begin{array}{l}31 \\
311\end{array}$ & $\begin{array}{l}17 \\
\cdots\end{array}$ & $\begin{array}{l}43 \\
\cdots\end{array}$ & $\dddot{i}$ & $\ddot{7}$ & $\begin{array}{l}21 \\
20\end{array}$ \\
\hline $\begin{array}{l}\mathrm{T} \\
\text { the } \\
\text { ben }\end{array}$ & hter & $\begin{array}{l}\text { ghest } \\
\text { rals }\end{array}$ & and & lowe & $\begin{array}{l}\text { est val } \\
\text { the } n\end{array}$ & $\begin{array}{l}\text { lue of } \\
\text { nean, }\end{array}$ & $\begin{array}{r}\mathrm{T} \\
\text { hen }\end{array}$ & sen & of ral & $\begin{array}{l}\text { an- } 15 \\
\text { an- }\end{array}$ & $37^{m}$ & is reje & ected, & & & & & & & \\
\hline & & & & & & & 1 & 23 & 17 & 39 & $\cdots$ & $\cdots$ & 22 & & & & & & & \\
\hline
\end{tabular}


Table for tite Tieduction of Tides. - Tro. 2.

Showing the Interval between the App. Time of the Moon's Superior Transit and the 'Time of Low Water, and also the Heights of Low Water, at Van Rensselaer Harbor, from Four Series of Observations made between Oetolser 10, 1853, and October 22, 1854.

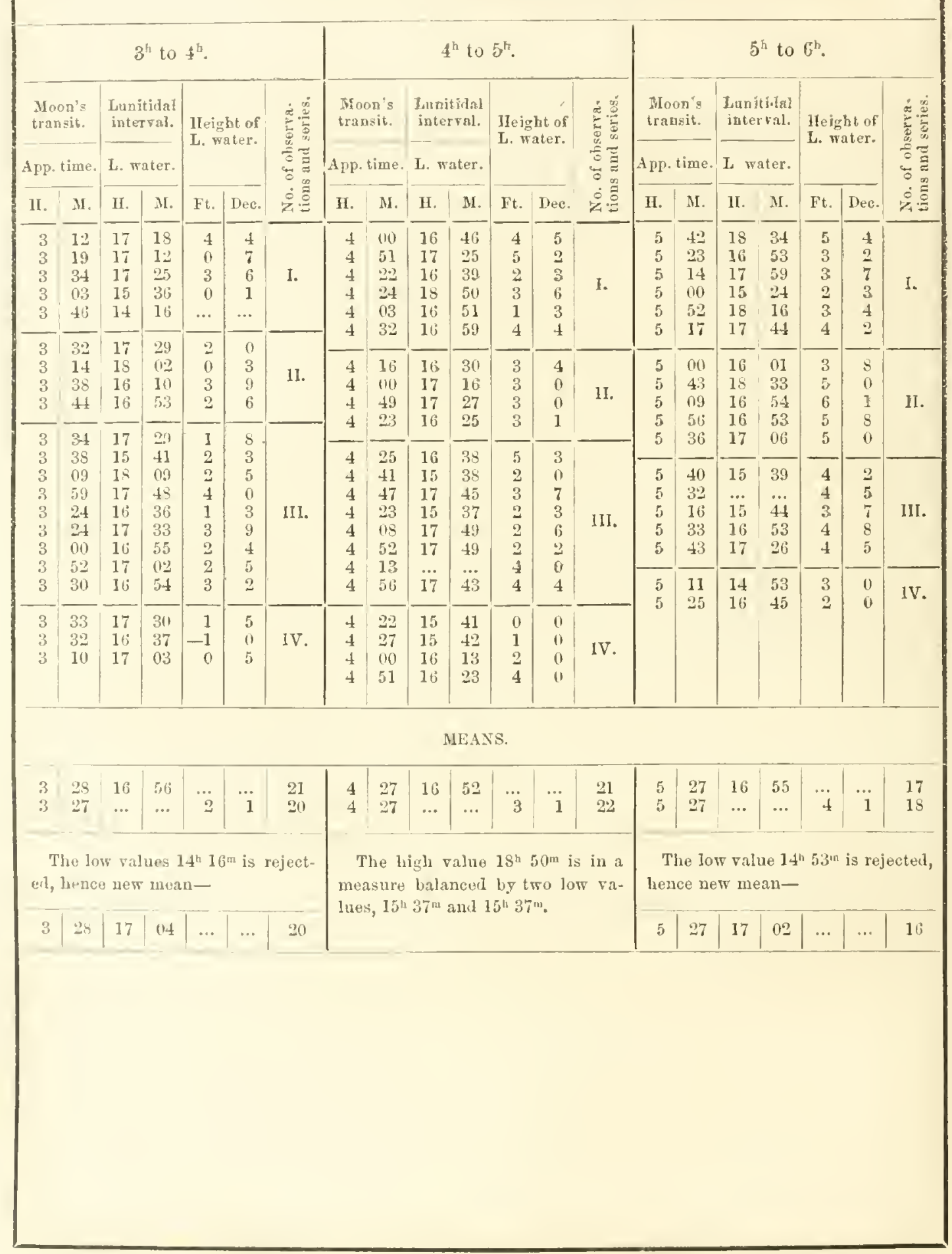


TAlLe for tie Reduction OF T'IDES.-No. 2.

Showing the Tuterval between the $\Lambda_{\mathrm{p}} \mathrm{p}$. Time of the Moon's Superior Transit and the Time of Low Water, and also the Heights of Jow Water, at Van Rensselaer Ilarbor, from Fonr Series of Observations made between Oetober 10, 1853, and Oetober 22, 1854.

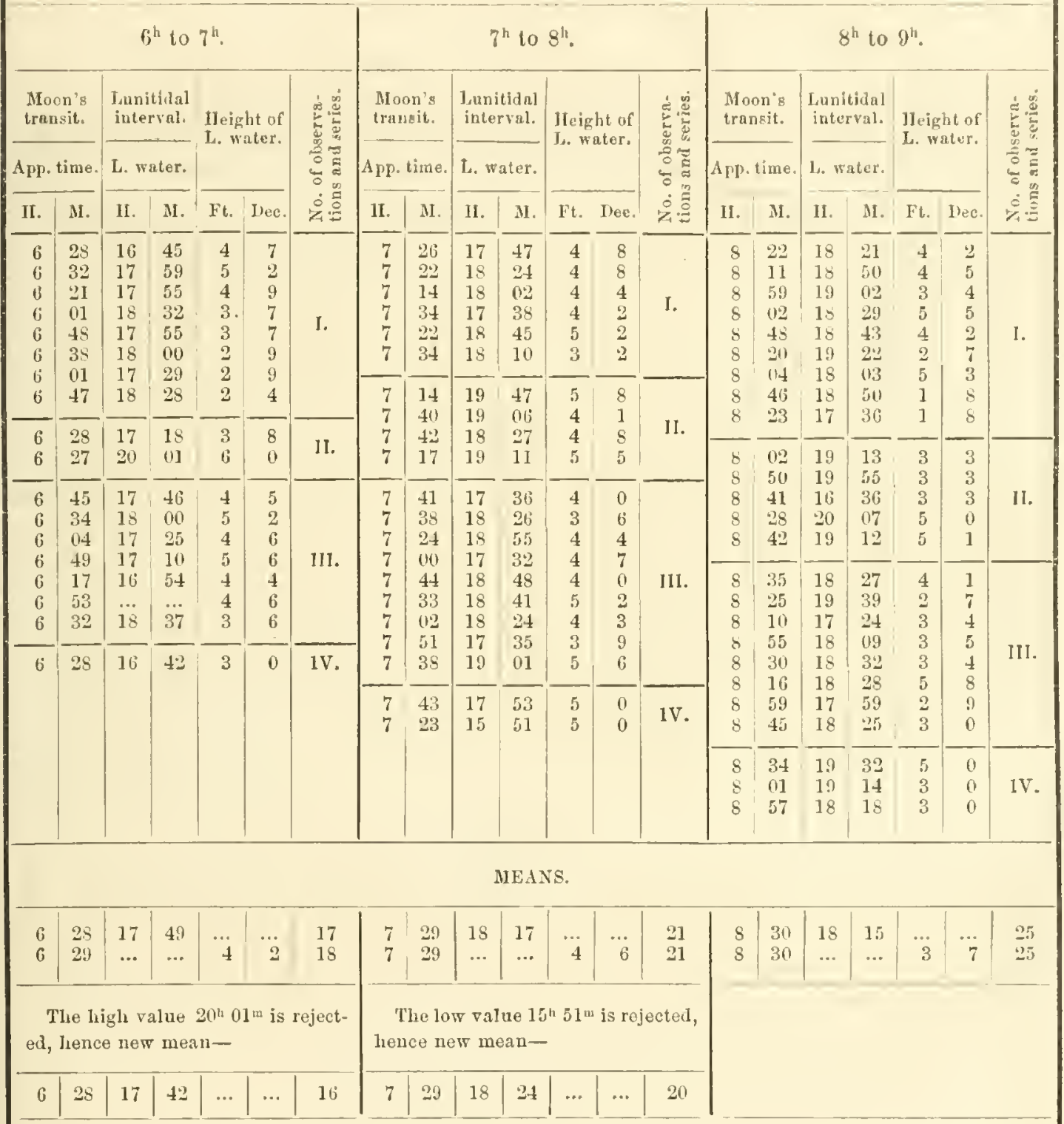


Table for the Reduction of Tides.-No. 2.

Showing the Interval between the App. Time of the Moon's Superior Transit and the Time of Low

Water, and also the Ileights of Low Water, at Van Rensselaer Harbor, from Four Series of Ouservations made between Oetober 10, 1853, and October 22, 1854.

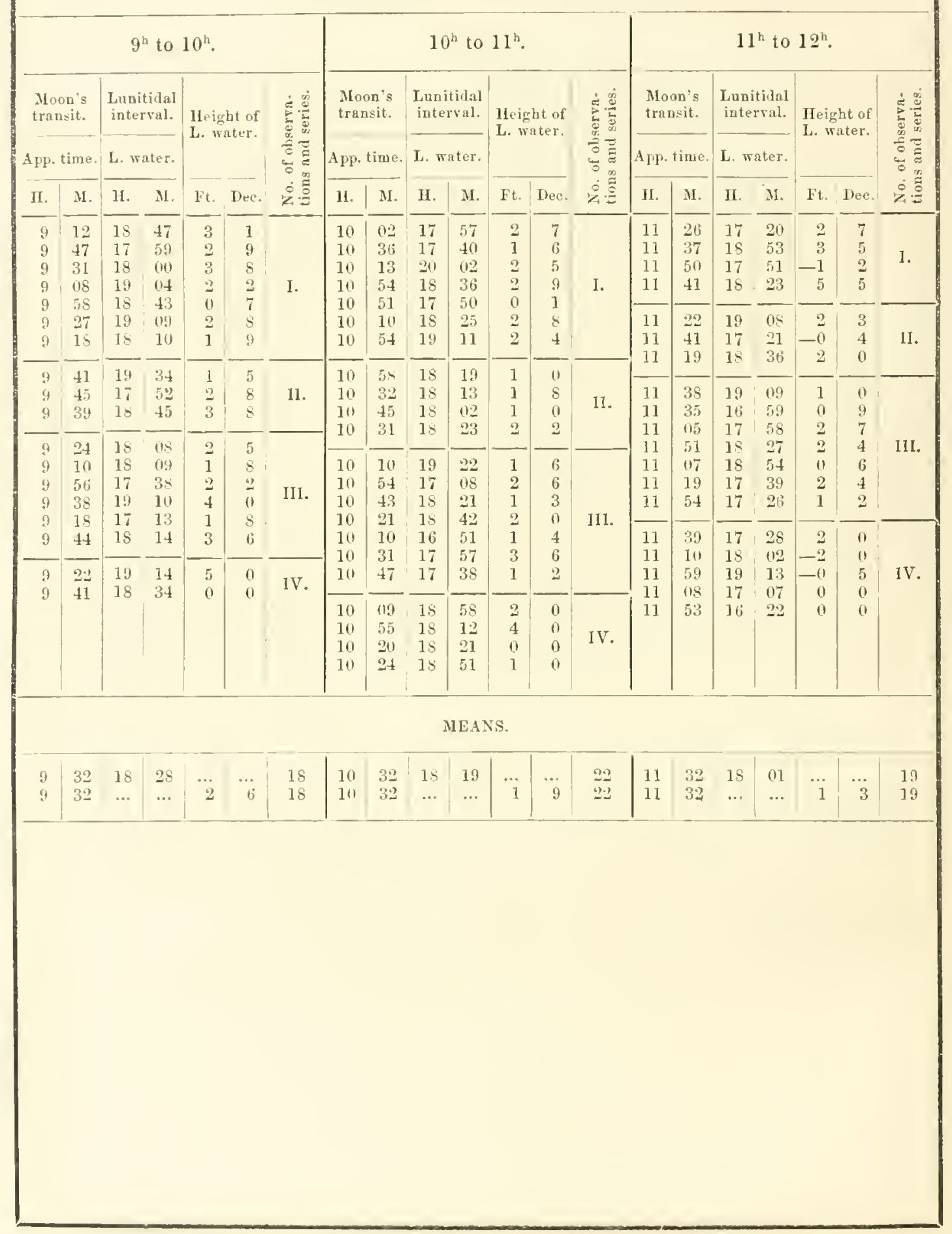


'TABLE FUR THE LiEUUCTION OF TIDES.-NO. 2.

Slowing the Interval between the Alp. 'Time of the Moon's Inferior 'Transit and the 'Time of Low Water, and also the IIeights of Low Water, at Van Rensselaer IIarbor, from I'our Series of Observations made between October 10, 1853, and Oetober 22, 1854.

\begin{tabular}{|c|c|c|c|c|c|c|c|c|c|c|c|c|c|c|c|c|c|c|c|c|}
\hline \multicolumn{7}{|c|}{$0^{\mathrm{h}}$ to $1^{\mathrm{h}}$. } & \multicolumn{7}{|c|}{$1^{\text {h }}$ to $2^{\mathrm{h}}$. } & \multicolumn{7}{|c|}{$2^{\mathrm{h}}$ to $3^{\mathrm{h}}$. } \\
\hline \multirow{2}{*}{\multicolumn{2}{|c|}{$\begin{array}{l}\text { Moon's } \\
\text { transit. } \\
\text { App. time. }\end{array}$}} & \multirow{2}{*}{\multicolumn{2}{|c|}{$\begin{array}{l}\text { Lunitida! } \\
\text { interval. } \\
\text { L. water. }\end{array}$}} & \multirow{2}{*}{\multicolumn{2}{|c|}{$\begin{array}{l}\text { Meight of } \\
\text { L. Witter. }\end{array}$}} & \multirow{3}{*}{ 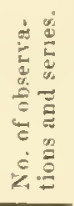 } & \multirow{2}{*}{\multicolumn{2}{|c|}{$\begin{array}{c}\begin{array}{c}\text { Moon's } \\
\text { tran-it. }\end{array} \\
\text { Appotime. }\end{array}$}} & \multirow{2}{*}{\multicolumn{2}{|c|}{$\begin{array}{c}\begin{array}{c}\text { Lunitidal } \\
\text { interval. }\end{array} \\
\text { L. water. }\end{array}$}} & \multirow{2}{*}{\multicolumn{2}{|c|}{$\begin{array}{l}\text { lleight of } \\
\text { L. water. }\end{array}$}} & \multirow{3}{*}{ 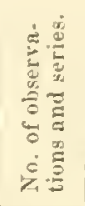 } & \multirow{2}{*}{\multicolumn{2}{|c|}{$\frac{\begin{array}{c}\text { Moon's } \\
\text { transit. }\end{array}}{\text { App time. }}$}} & \multirow{2}{*}{\multicolumn{2}{|c|}{$\begin{array}{l}\text { Imnitidnl } \\
\text { interval. } \\
\text { L. water. }\end{array}$}} & \multirow{2}{*}{\multicolumn{2}{|c|}{$\begin{array}{l}\text { Height of } \\
\text { L. water. }\end{array}$}} & \multirow{3}{*}{ 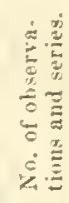 } \\
\hline & & & & & & & & & & & & & & & & & & & & \\
\hline II. & M. & 11. & M. & $\mathrm{Ft}$. & Dec. & & II. & M. & 11. & M. & Ft. & Dec. & & $1 \mathrm{I}$. & MI. & 11. & $M$. & $\mathrm{Ft}$. & Dec. & \\
\hline 0 & 35 & 17 & 25 & 1 & 8 & \multirow{6}{*}{ I. } & 1 & 18 & 17 & 27 & 1 & 6 & \multirow{6}{*}{ I. } & 2 & $n 2$ & 15 & 43 & 5 & 7 & \multirow{6}{*}{1.} \\
\hline 0 & 48 & 15 & 28 & 0 & 1 & & 1 & 46 & 19 & 00 & 1 & 5 & & 2 & 45 & 16 & 57 & 2 & $\dot{s}$ & \\
\hline 0 & 44 & 18 & $16^{\circ}$ & 1 & 3 & & 1 & $3 t$ & 17 & 44 & -0 & 1 & & 2 & 47 & 17 & 14 & $\overline{1}$ & $\theta$ & \\
\hline 0 & 21 & 18 & 05 & -0 & 3 & & 1 & 25 & 17 & 45 & 0 & 0 & & 2 & 211 & 16 & 5.4 & 1 & 9 & \\
\hline 0 & 06 & 16 & 58 & 3 & 4 & & \multirow[t]{2}{*}{1} & \multirow{2}{*}{44} & \multirow{2}{*}{17} & 19 & $\cdots$ & \multirow{2}{*}{$\ldots$} & & \multirow{2}{*}{$\begin{array}{l}2 \\
2\end{array}$} & $3 n$ & 15 & 10 & -0 & 1 & \\
\hline 0 & 55 & 17 & 08 & 2 & 7 & & & & & 10 & + & & & & 34 & 17 & 58 & $\cdots$ & $\cdots$ & \\
\hline 0 & 31 & 19 & 31 & -1 & 8 & & 1 & 21 & 17 & 25 & 3 & 2 & & 2 & 20 & 17 & 56 & 1 & 5 & \\
\hline 1$)$ & 33 & 18 & 13 & 2 & 4 & & 1 & 45 & 19 & 02 & 1 & 3 & II & 2 & 06 & 17 & 40 & 2 & 2 & \\
\hline 0 & 07 & 17 & 55 & -0 & 9 & & 1 & 38 & 18 & 43 & 0 & 7 & 11. & 3 & $5 I$ & 16 & 25 & 3 & 9 & 11. \\
\hline 0 & 57 & 18 & 05 & 1 & 1 & 11. & 1 & 14 & 18 & 27 & 1 & 3 & & 2 & 31 & 18 & 47 & 1 & 9 & \\
\hline 0 & $\begin{array}{l}116 \\
52\end{array}$ & $\begin{array}{l}18 \\
19\end{array}$ & $\begin{array}{l}15 \\
29\end{array}$ & 3 & $\begin{array}{l}3 \\
2\end{array}$ & & 1 & 59 & 18 & 57 & 3 & 3 & & 2 & 26 & 18 & 56 & 1 & 6 & \\
\hline 0 & 29 & 17 & 56 & -0 & 2 & & 1 & 33 & 16 & 00 & 1 & 5 & & 2 & 20 & 14 & 57 & 1 & 7 & \\
\hline & & & & & & & 1 & 04 & 15 & 4 & 2 & 3 & & 2 & (1) & 17 & 33 & 1 & 7 & \\
\hline 0 & $\begin{array}{l}00 \\
45\end{array}$ & 17 & 10 & $\begin{array}{l}2 \\
2\end{array}$ & 2 & & 1 & $\begin{array}{l}54 \\
40\end{array}$ & 17 & 24 & 1 & 6 & 111. & 2 & 44 & 16 & 14 & 2 & 5 & \\
\hline 0 & $\begin{array}{l}45 \\
15\end{array}$ & $\begin{array}{l}18 \\
16\end{array}$ & 03 & 0 & $\begin{array}{l}0 \\
5\end{array}$ & & 1 & 49 & 16 & 41 & 0 & 2 & & 2 & 52 & 16 & 3 & 1 & i; & 111. \\
\hline 0 & $\begin{array}{r}15 \\
+3\end{array}$ & $\begin{array}{l}10 \\
17\end{array}$ & $\begin{array}{l}13 \\
45\end{array}$ & 1 & 5 & 111. & 1 & 24 & 18 & 03 & 3 & 0 & & $\begin{array}{l}2 \\
0 \\
0\end{array}$ & 13 & 17 & 14 & 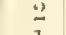 & 5 & \\
\hline $\begin{array}{l}0 \\
0\end{array}$ & $\begin{array}{l}+5 \\
35\end{array}$ & 17 & 52 & 2 & 4 & & & 31 & 17 & 24 & 1 & 0 & & 2 & $\begin{array}{l}31 \\
?+4\end{array}$ & 17 & 119 & 1 & 0 & \\
\hline $\begin{array}{l}0 \\
0\end{array}$ & 27 & 17 & 58 & 1 & 5 & & 1 & $30^{\circ}$ & 18 & 213 & & 0 & & 2 & 211 & 17 & 25 & 2 & 0 & \\
\hline & & & & & & & 1 & 29 & 17 & 39 & I & 0 & & 2 & 24 & 17 & 09 & -1 & 0 & \\
\hline 0 & 47 & I 6 & 45 & -1 & 7 & & 1 & 09 & 19 & 0.3 & -1 & 0 & IV. & 2 & 15 & 17 & 53 & 0 & 0 & IV. \\
\hline 0 & 00 & 17 & 07 & 1 & 0 & & 1 & 57 & 16 & $46^{\circ}$ & 0 & 0 & & 2 & $\$ 5$ & 15 & 28 & 0 & 0 & \\
\hline 0 & 45 & 17 & 53 & 0 & 0 & IV. & & & & & & & & & & & & & & \\
\hline 0 & 22 & 18 & 20 & 1 & 0 & & & & & & & & & & & & & & & \\
\hline 0 & 17 & 16 & 58 & 0 & 0 & & & & & & & & & & & & & & & \\
\hline
\end{tabular}

MEANS.

\begin{tabular}{c|c|c|c|c|c|c|c|c|c|c|c|c|c|c|c|c|c|c|c|c|c|}
0 & 30 & 17 & 50 & $\ldots$ & $\ldots$ & 24 & 1 & 33 & 17 & 52 & $\ldots$ & $\ldots$ & 21 & 2 & 29 & 17 & 09 & $\ldots$ & $\ldots$ & 21 \\
0 & 30 & $\ldots$ & $\ldots$ & 1 & 1 & 24 & 1 & 33 & $\ldots$ & $\ldots$ & 1 & 1 & 20 & 2 & 28 & $\ldots$ & $\ldots$ & 1 & 7 & 20 \\
\hline
\end{tabular}

The tro higl aul two low values of the intervals nearly balance in

The value $15^{\mathrm{h}} 4^{\mathrm{m}}$ is rejected,

The lowest value $14^{\mathrm{h}} 33^{\mathrm{m}}$ is rethe mean. jected, lience-

\begin{tabular}{l|l|l|l|l|l|l|l|l|l|l|l|l|l}
1 & 34 & 17 & 58 & $\ldots$ & $\ldots$ & 20 & 2 & 28 & 17 & 15 & $\ldots$ & $\ldots$ & 20
\end{tabular}


Table for tile Renuetion of Tines.-No. 2.

Showing the Intersal between the I pp. Time of the Moon's Inferior Tramsit and the Time of Low

Water, and also the Ileights of Low Water, at Tan Rensselaer IIarbor, from Four Series of Observations made between October 10, 1853, and October 22, 1854 .

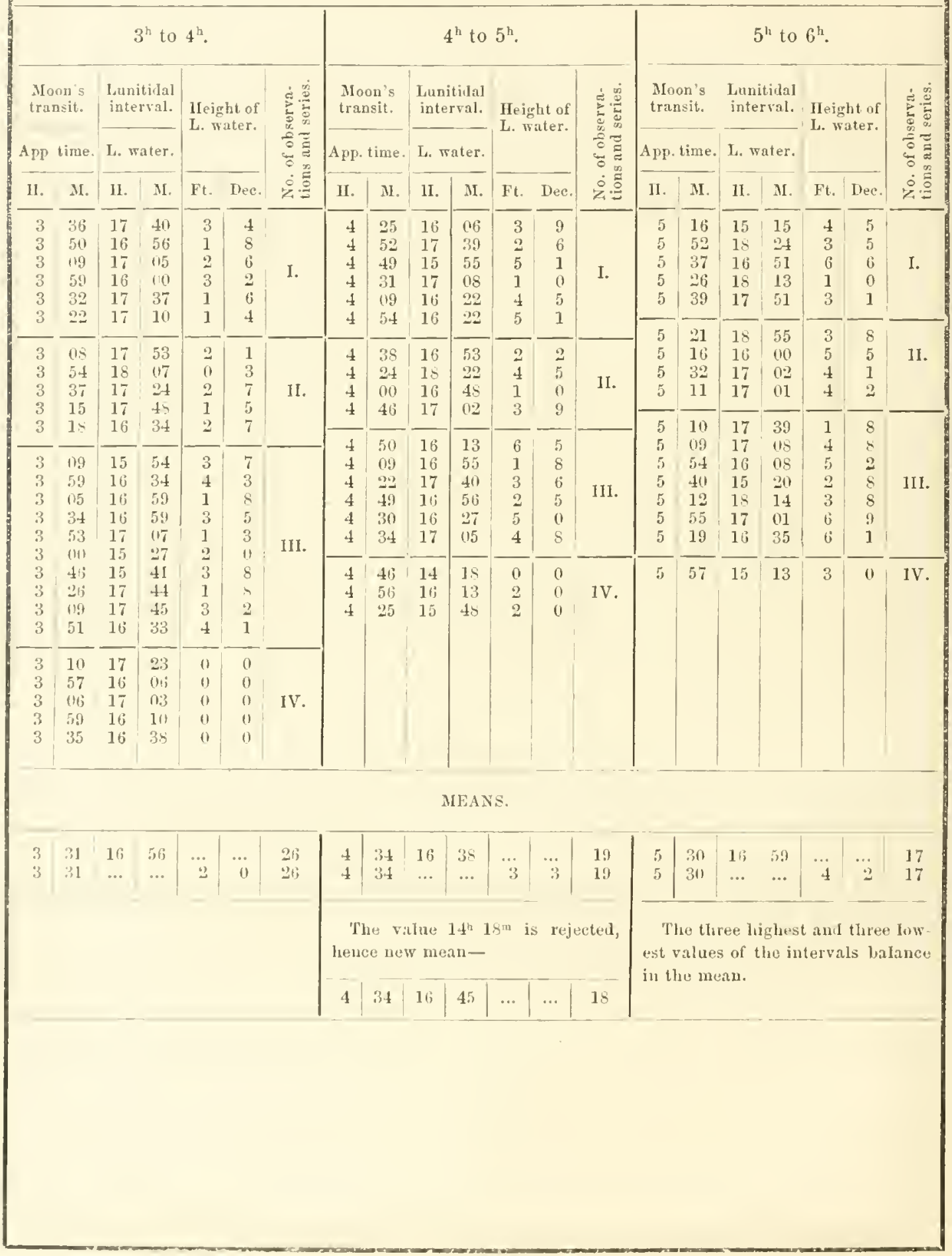


'Table for the Reduction of Tides.-No. 2.

Showing the Interral between the App. Time of the Moon's Inferior Transit and the Time of Low Water, and also the IIcights of Iow Water, at Tan Renssclaer Ilarbor, from Four Series of Observations made between Oetober 10, 1853, and Oetober 22, 1854.

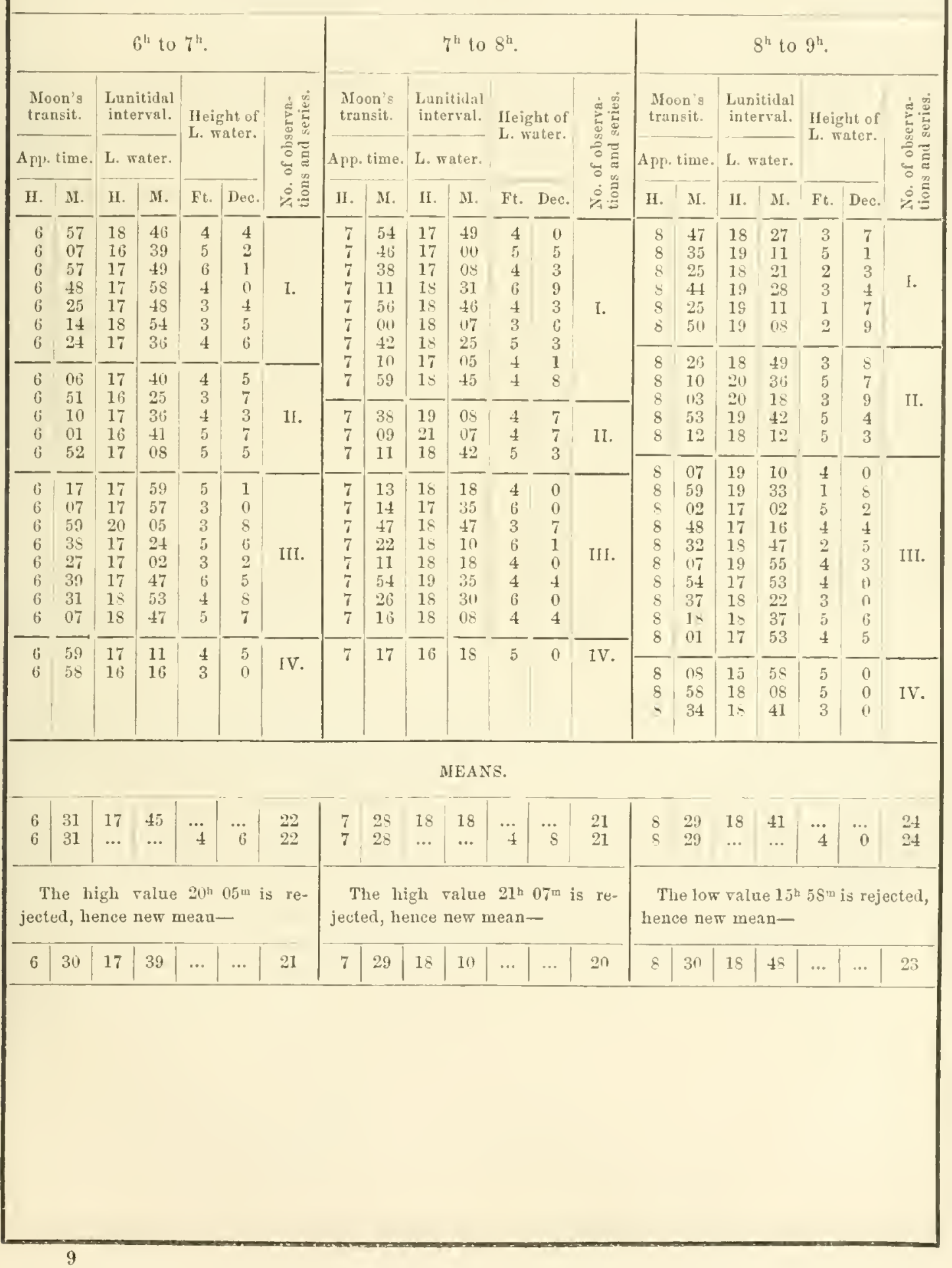


Table for tile Reduction of Tides.-No. 2.

Showing the Interval between the App. Time of the Moon's Inferior Transit and the Time of Low Water, and also the Ileigbts of Low Water, at Van Rensselaer Harbor, from Four Series of Obserrations made between October 10, 1853, to October 22, 1855.

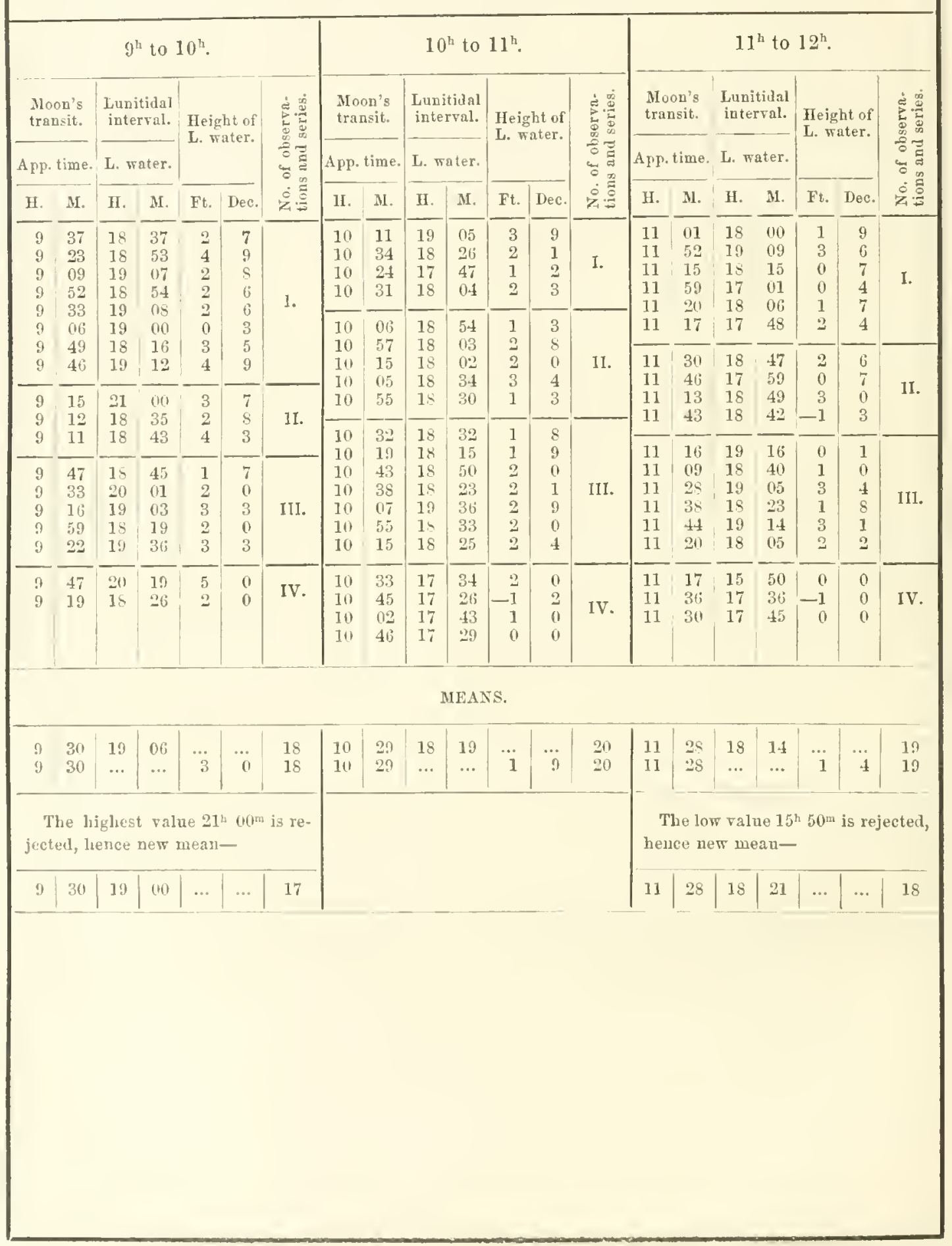


'The preceding tables (No. 2) contain the individual and mean values for interval and height, for high and low water, and the moon's upper and lower transit. 'The mean, in some cases, was improved by the ipplication of Peirce's criterion for the rejection of doubtful observations; a few other rejections were made, as stated, in order to obtain a well-balanced mean; of 982 observations of the interval, but 17 were thus rejected.

Half-monthly Inequality.-For the comparison of the observed with the theoretical values, it is customary to use the forms of the equilibrium theory or of the wave theory, ${ }^{1}$ certain modifications being necessary to produce an agreement between these theories with observation. According to the equilibrium theory the formula for the position of the pole of the tidal spheroid is:

$$
\tan .2 \theta^{\prime}=-\frac{h \sin .2 \phi}{h^{\prime}+h \cos .2 \phi},
$$

where $h$ and $h^{\prime}$ are the clevations of the spheroid due to the sum and moon respectively, $\phi$ the angular distance of the moon from the sum and $\theta^{\prime}$ the angular distance of the pole of the spheroid (or of high water) from the moon's place. In reality; however, the pole of this spheroid follows the moon at a certain distance, the mean value $\lambda$ ' of which is known as the "mean establishment" (also fundamental hour, corrected establishment), and which corresponds to a distance of the sun and moon of $\phi-\alpha$ instead of $\phi$. 'This retroposition of the theoretical tide has becn called the age of the tide. For the comparison of the observed and computed ralues for the half-monthly incquality in time, we have the formula: ${ }^{2}$

$$
\tan .2\left(\theta^{\prime}-\lambda^{\prime}\right)=-\frac{h \sin .2(\phi-\alpha)}{h^{\prime}+h \cos .2(\phi-\alpha)^{\circ}} .
$$

This inequality goes through its period twice in each month. Proper values have to be found for the ratio $\frac{h}{h^{\prime}}$ and the angle $\alpha$.

The observations of 480 high uaters furnish us with the following values, derived from the preceding tabulation on form No. 2:-

1 An account of the Equilibrium, Laplace's and the Ware Theories, will be found in the Encyelopredia of Astronomy, forming a portion of the Fneyclopæedia Metropolitana, London, 1848; article "Ou Tides and Wares," by G. B. Airy, Esq., Astronomer Royal.

${ }^{2}$ Phil. Trans. Royal Society, 1834, Part I. On the Empirical Laws of the Tides in the Port of London, with some Refleetions on the Theory; by the Riev. W. Whewell.

See also Phil. Trans. Royal Society, 1836, Part I. Researehes on the Tides, fourth series: On the Empirical Laws of the Tides in the Port of Lirerpool. By the Rev. W. Wherell. 


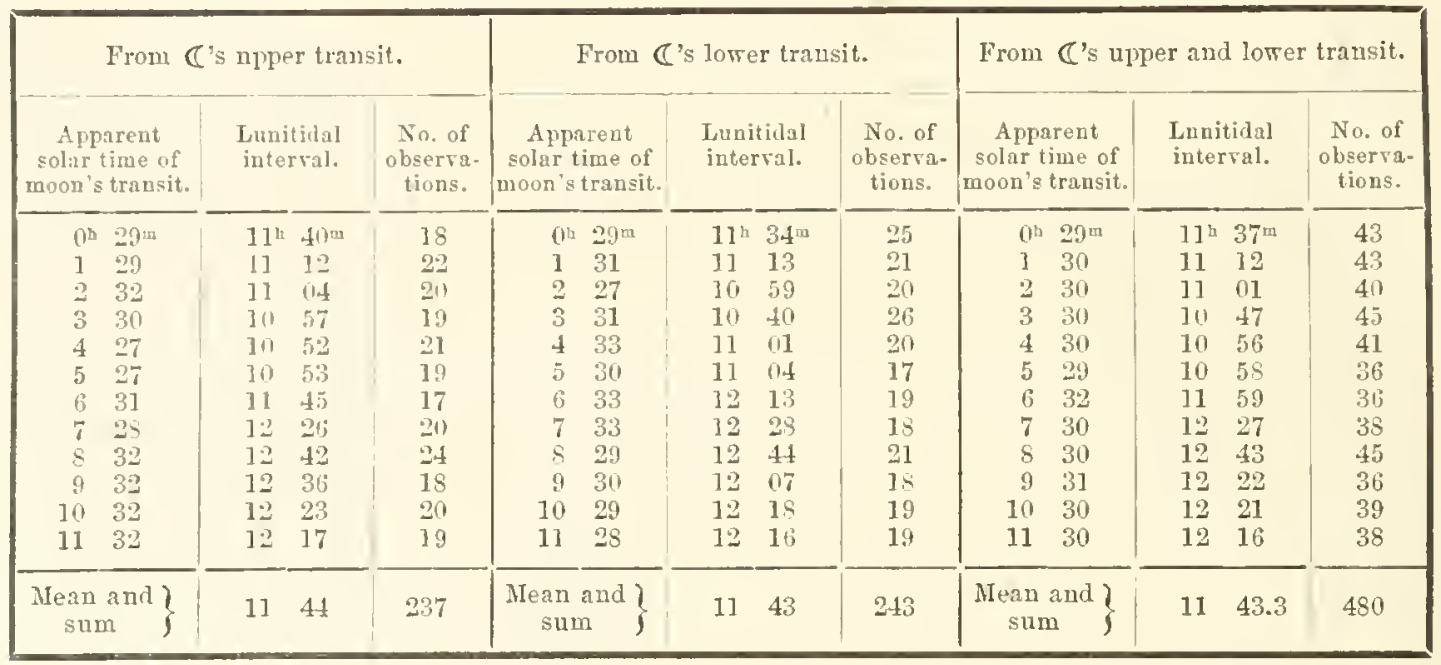

'The nean establishment resulting from the observed times of 480 high waters at Van Rensselner Harbor is therefore $11^{\text {h }} 43.3^{\text {m }}$, referred to the moon's transit immediately preceding and corresponding to a mean horizontal parallax of the moon and sum, and to the moon's and sun's declination of $16^{\circ}$ nearly. The mean interval corresponds to the moon's transit of $\left(0^{\mathrm{h}} \cdot 21^{\mathrm{m}}\right.$ nearly, indicating that the epoch would lave come out $0^{\mathrm{h}} 0^{\mathrm{m}}$ if transit $\mathrm{E}$ ( Tides, by J. W. Lubback, Esq., Loncion, 1839) or that immediately preceding transit $\mathrm{F}$ had been used.

In like manner we obtain the following table from the observed times of 485 low waters at Van Rensselaer Harbor:-

\begin{tabular}{|c|c|c|c|c|c|c|c|c|}
\hline \multicolumn{3}{|c|}{ From C's upper transit. } & \multicolumn{3}{|c|}{ From $\mathbb{C}$ 's lower transit. } & \multicolumn{3}{|c|}{ From $\mathbb{C}$ 's upper and lower transit. } \\
\hline $\begin{array}{l}\text { Apparent } \\
\text { solar time of } \\
\text { moon's transit. }\end{array}$ & $\begin{array}{l}\text { Lunitidal } \\
\text { interval. }\end{array}$ & $\begin{array}{c}\text { No. of } \\
\text { obserra. } \\
\text { tions. }\end{array}$ & $\begin{array}{c}\text { Apparent } \\
\text { solar time of } \\
\text { moon's transit. }\end{array}$ & $\begin{array}{l}\text { Lunitidal } \\
\text { interval. }\end{array}$ & $\begin{array}{c}\text { No. of } \\
\text { cliserra- } \\
\text { tions. }\end{array}$ & $\begin{array}{c}\text { Apparent } \\
\text { solar time of } \\
\text { moon's transit. }\end{array}$ & $\begin{array}{l}\text { Lunitilal } \\
\text { interral. }\end{array}$ & $\begin{array}{l}\text { No. of } \\
\text { observa- } \\
\text { tions. }\end{array}$ \\
\hline $0 \mathrm{n} 30 \mathrm{~m}$ & $17^{\mathrm{h}} 4^{43^{\mathrm{m}}}$ & 21 & $0^{\mathrm{h}} 30^{\mathrm{m}}$ & $17^{\mathrm{m}} 50^{\mathrm{m}}$ & 24 & $0^{\mathrm{h}} \quad 30^{\mathrm{m}}$ & $17^{\mathrm{h}} 50^{\mathrm{m}}$ & +5 \\
\hline $1: 8$ & $17 \quad 39$ & $2 \cdot 2$ & 134 & $17 \quad 58$ & 20 & 131 & $17 \quad 45$ & 42 \\
\hline $2 \quad 31$ & $17 \pm 3$ & 21 & 223 & $17 \quad 15$ & 30 & $2 \quad 30$ & 1729 & 41 \\
\hline $32 x$ & 170.4 & 20 & $3 \quad 31$ & 1656 & 26 & $3 \quad 30$ & $16 \quad 59$ & 46 \\
\hline $4 \quad 27$ & $16 \quad 52$ & 21 & 434 & $16 \quad 45$ & 18 & +30 & $16 \quad 49$ & 39 \\
\hline $5 \quad 27$ & $\begin{array}{ll}17 & 022\end{array}$ & 16 & 30 & $16 \quad 59$ & 17 & $5 \quad 29$ & $17 \quad 00$ & 33 \\
\hline (i) 23 & 17 & $16^{\circ}$ & 30 & 17 & 21 & (i) 30 & 1740 & 37 \\
\hline $7 \quad 24$ & Is & 20 & 29 & 15 & 20 & $7 \quad 29$ & is $\quad 17$ & 40 \\
\hline 30 & $15 \quad 15$ & 25 & S $\quad 29$ & 15 & 23 & $8 \quad 30$ & is 31 & 48 \\
\hline ? $32 \underline{z}$ & $1=28$ & Is & 30 & 19 (10 & 17 & ? 31 & 1543 & 35 \\
\hline $10 \quad: 3$ & $1 \times \quad 19$ & 22 & $10 \quad 29$ & $18 \quad 19$ & 20 & $10 \quad 30$ & $18 \quad 19$ & $4: 3$ \\
\hline $113: 2$ & ] $=11$ & 10 & 1125 & Is 21 & 18 & 1130 & $\begin{array}{ll}18 & 11\end{array}$ & 37 \\
\hline $\left.\begin{array}{l}\text { Mean and } \\
\operatorname{sum}\end{array}\right\}$ & $17 \quad 40$ & 211 & $\left.\begin{array}{c}\text { Mean and } \\
\text { sum }\end{array}\right\}$ & $17 \quad 50$ & $24 i$ & $\left.\begin{array}{l}\text { Menn and } \\
\text { sum }\end{array}\right\}$ & 1745.0 & $4 \varsigma 5$ \\
\hline
\end{tabular}

'The mean establishment resulting from the observed times of 485 low waters is $17^{14} 48.0^{\mathrm{m}}$, referred to the moon's transit immediately preceding low water, and the same to which the preceding ligh water has been referred; the difference between the two mean intervals is $6^{\text {h }} 04.7^{\mathrm{m}}$.

Iro obtain a mumerieal expression for the half-monthly inequality in time, the value for a should be determined so as to furnish, in particular, good results for 
$5^{\mathrm{h}} 30^{\mathrm{m}}, 6^{\mathrm{h}} 30^{\mathrm{m}}, 7^{\mathrm{h}} 30^{\mathrm{m}}$, where the curve is stecpest; the value $\frac{h}{h^{\prime}}$ is obtainerl from the greatest range of the inequality determined, for a first approximation, by a graphical process. I find from the observed high waters $\alpha=0^{\mathrm{h}} 21^{\text {tn }}$ or $5^{\circ} 15^{\prime}$, and from the low waters $\alpha=0^{\mathrm{h}} 50^{\mathrm{m}}$ or $12^{\circ} 30^{\prime}$. liange of inequality, from the high waters, $1^{\text {h }} 51^{\mathrm{m}}$ or $27^{\circ} 48^{\prime}$, the $\sin$. of which is 0.4649 , and for the low waters, range $1^{\mathrm{h}} 54^{\mathrm{m}}$ or $28^{\circ} 30^{\prime}$, the $\sin$. of which is 0.4771 ; hence the expression for the halfmonthly inequality in time becomes

From the observed high waters $\tan .2\left(0^{\prime}-175^{\circ} 49^{\prime} .5\right)=-\frac{0.4649 \sin .2\left(\phi-5^{\circ} 15^{\prime}\right)}{1+0.4649 \cos 2\left(\phi-5^{\circ} 15^{\prime}\right)}$

“ “ " low waters $\tan .2\left(\theta^{\prime}-267^{\circ} 00^{\prime}\right)=-0.4771 \sin .2\left(\phi-12^{\circ} 30^{\prime}\right)$ $1+0.47 \pi 1 \cos .2\left(\phi-12^{\circ} 30^{\prime}\right)$

These expressions furnish us with the following comparison:-

HALF-MONTHLY INEQUALITY IN TIME.

\begin{tabular}{|c|c|c|c|c|c|c|c|}
\hline \multicolumn{4}{|c|}{ From high waters. } & \multicolumn{4}{|c|}{ From low waters. } \\
\hline $\begin{array}{l}\text { Apparent } \\
\text { solar time of } \\
\text { moon's transit. }\end{array}$ & Observed. & Computed. & Difference. & $\begin{array}{c}\text { Apparent } \\
\text { solar time of } \\
\text { moon's transit. }\end{array}$ & Observed. & Computed. & Difference. \\
\hline $\begin{array}{cl}\left(0^{\mathrm{h}}\right. & 29 \mathrm{~m} \\
1 & 30 \\
2 & 30 \\
3 & 30 \\
4 & 30 \\
5 & 29 \\
6 & 32 \\
7 & 30 \\
\mathrm{~S} & 30 \\
9 & 31 \\
10 & 30 \\
11 & 30\end{array}$ & $\begin{array}{l}-6^{\mathrm{m}} \\
-31 \\
-42 \\
-56 \\
-47 \\
-45 \\
+16 \\
+44 \\
+60 \\
+39 \\
+3 \mathrm{~S} \\
+33\end{array}$ & $\begin{array}{l}-3^{\text {m }} \\
-22 \\
-39 \\
-52 \\
-55 \\
-38 \\
+8 \\
+46 \\
+56 \\
+48 \\
+34 \\
+16\end{array}$ & $\begin{array}{l}-3^{\mathrm{w}} \\
-9 \\
-3 \\
-4 \\
+8 \\
-7 \\
+8 \\
-2 \\
+4 \\
+9 \\
+4 \\
+17\end{array}$ & $\begin{array}{rl}0 & 30 \\
1 & 31 \\
2 & 30 \\
3 & 30 \\
4 & 30 \\
5 & 29 \\
6 & 30 \\
7 & 29 \\
8 & 30 \\
9 & 31 \\
10 & 30 \\
11 & 30\end{array}$ & $\begin{array}{r}+2 \mathrm{~m} \\
0 \\
-19 \\
-49 \\
-59 \\
-45 \\
-8 \\
+29 \\
+43 \\
+55 \\
+31 \\
+23\end{array}$ & $\begin{array}{l}+6^{m} \\
-13 \\
-31 \\
-47 \\
-56 \\
-52 \\
-12 \\
+33 \\
+56 \\
+54 \\
+43 \\
+25\end{array}$ & $\begin{array}{l}-4^{m} \\
+13 \\
+12 \\
-2 \\
-3 \\
+4 \\
+10 \\
-4 \\
-13 \\
+1 \\
-11 \\
-2\end{array}$ \\
\hline
\end{tabular}

Considering that the times of high and low water are only obscrved to the nearest half hour and for some time to the nearest hour, the agreement as shown above and by the diagrams, seems to be satisfactory.

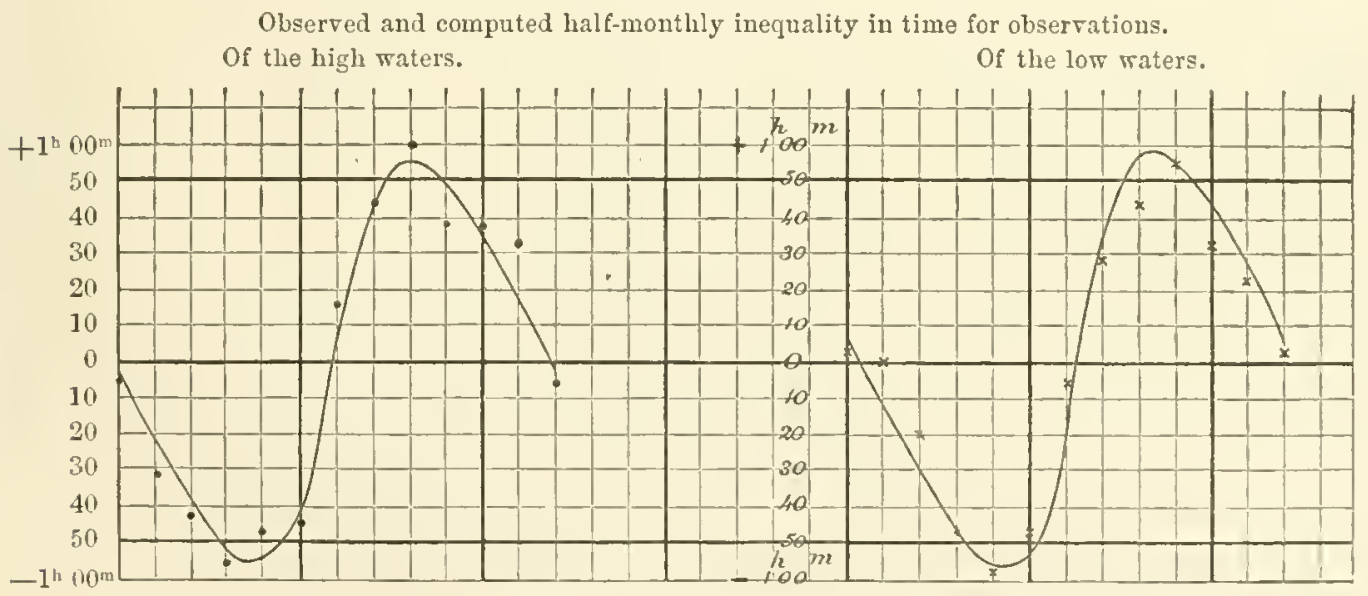

$\left(0^{\mathrm{h}} 1 \quad 2 \quad 3 \quad 4 \quad 5 \quad 6 \quad 7 \quad 5 \quad 9 \quad 101112 \mathrm{~h}\right.$

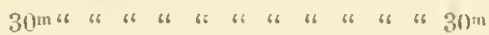

(th $123456789101112 \mathrm{~b}$ $30 \mathrm{~m}$ " 
In the above diagram, the observed values are indicated by dots; the computed values are represented by curves. From the times we have seen the mean value $\frac{h}{h^{\prime}}\left(\right.$ or $^{\prime} \frac{S^{\prime \prime}}{I^{\prime \prime}}$ of the ware theory and $(A)$ of Lubbock's $)=0.471$, and $\alpha=0^{\mathrm{b}} 36^{\mathrm{m}}$; hence, the age of the tide, or the time requisite for the moon to increase its right ascension by that amount, becomes $\frac{3}{4} \frac{6}{8}$ days, or 18 hours.

Half-monthly Inequality in Height.-The theoretical expression for the half monthly inequality in height of high water is:

$$
n=\sqrt{ }\left\{h^{\prime 2}+h^{2}+2 h^{\prime} h \cos .2 \phi\right\}^{1}
$$

where $n$ expresses the height of the pole of the equilibrium spheroid above the mean level of the surface; for its application, and according to the wave theory, it must be changed to:

$$
\eta=\sqrt{ }\left\{h^{2}+h^{2}+2 h^{\prime} h \cos .2(\phi-\alpha)\right\}^{2}
$$

The following table contains the results of the observations from the high and low waters, and the moon's superior and inferior transit:

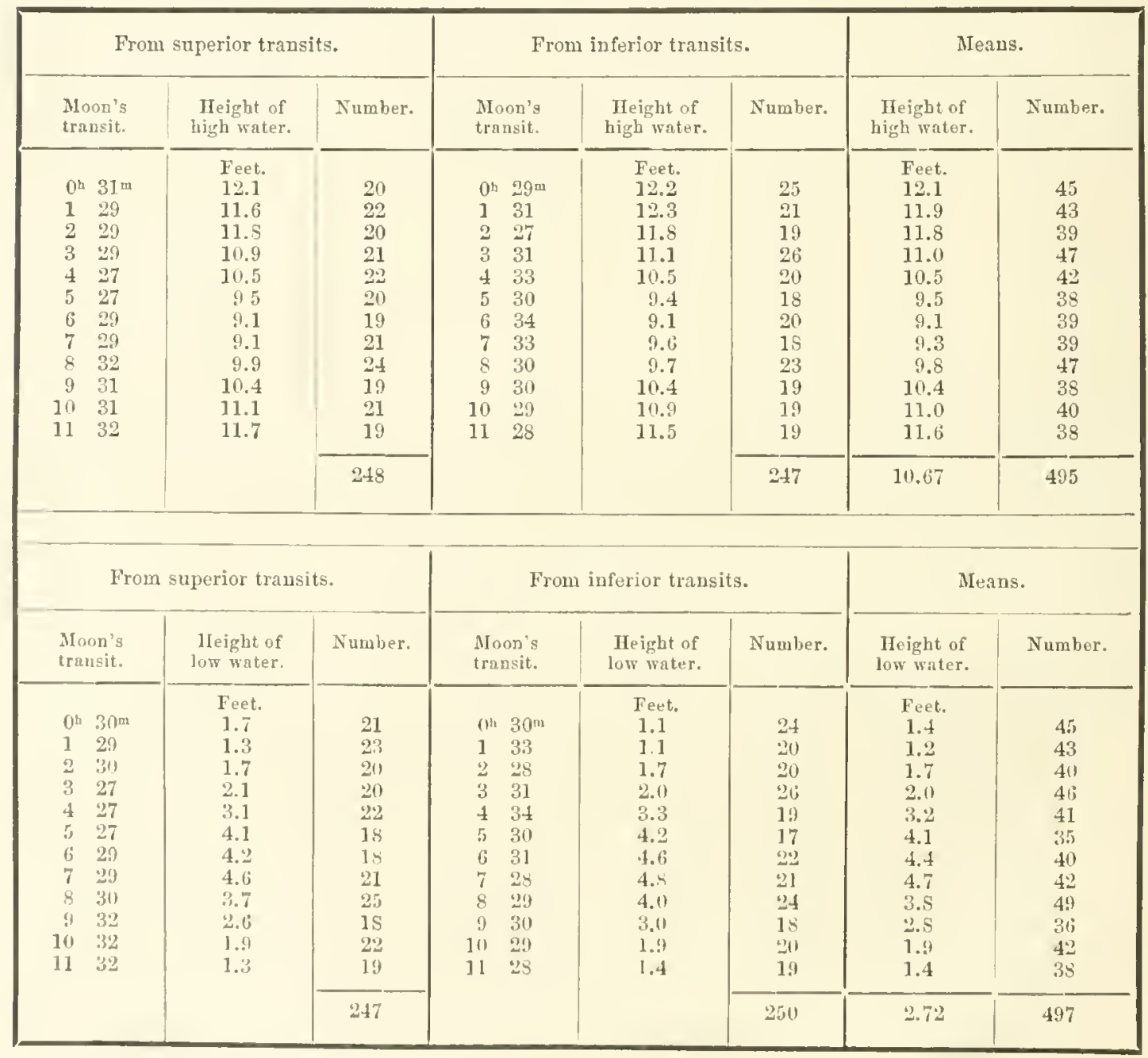

2 See l'hil. 'Trans. Royal Soc., 1834 and 1836.

* Encyclopedia Mctropolitana, Tides and Wares, Art. (535). The expression given ly Mr. Lub. lock is:

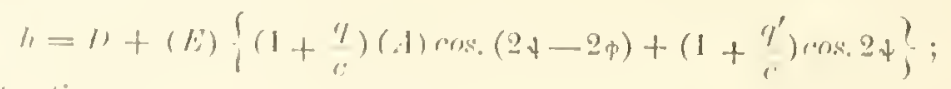


The values for $h^{\prime}, h$ and $\alpha$ were found from the maxima and minima values of the inequality, viz., for the high water:

$$
y=\sqrt{ }\left\{10.6^{2}+1.5^{2}+31.8 \cos .2\left(\phi-15^{\circ}\right)\right\}
$$

for the low waters:

$$
y^{\prime}=\sqrt{ }\left\{2.95^{2}+1.75^{2}-5.16 \cos .2(\phi-15)\right\}
$$

'These cxpressions may be changed to

$$
y=10.6+1.5 \cos .2\left(\phi-15^{\circ}\right), \text { and } y^{\prime}=2.7-1.7 \cos .2\left(\phi-15^{\circ}\right)
$$

\begin{tabular}{|c|c|c|c|c|c|c|}
\hline \multirow[b]{2}{*}{ Moon's transit. } & \multicolumn{3}{|c|}{ Ileight of high water. } & \multicolumn{3}{|c|}{ Ileight of low water. } \\
\hline & Computesl. & Obserred. & Difference. & Computẻ. & Ohserved. & Difference. \\
\hline $\begin{array}{cl}0 \text { ㄴ } & 30 \mathrm{~m} \\
1 & 30 \\
2 & 30 \\
3 & 30 \\
4 & 30 \\
5 & 30 \\
6 & 30 \\
7 & 30 \\
8 & 30 \\
9 & 30 \\
10 & 30 \\
11 & 30\end{array}$ & $\begin{array}{r}12.1 \\
12.0 \\
11.7 \\
11.0 \\
111.2 \\
9.5 \\
9.1 \\
9.2 \\
9.5 \\
10.2 \\
11.0 \\
11.7\end{array}$ & $\begin{array}{r}12.1 \\
11.9 \\
11.8 \\
11.0 \\
10.5 \\
9.5 \\
9.1 \\
9.3 \\
9.8 \\
10.4 \\
11.0 \\
11.6\end{array}$ & $\begin{array}{r}0.0 \\
-0.1 \\
+\quad 1.1 \\
0.0 \\
+0.3 \\
11.0 \\
0.0 \\
+0.1 \\
+0.3 \\
+0.2 \\
0.0 \\
-0.1\end{array}$ & $\begin{array}{l}1.1 \\
1.1 \\
1.5 \\
2.3 \\
3.1 \\
3.9 \\
4.3 \\
4.3 \\
3.9 \\
3.1 \\
2.3 \\
1.5\end{array}$ & $\begin{array}{l}1.4 \\
1.2 \\
1.7 \\
2.0 \\
3.2 \\
4.1 \\
4.4 \\
4.7 \\
3.8 \\
2.8 \\
1.9 \\
1.4\end{array}$ & $\begin{array}{l}+0.3 \\
+11.1 \\
+0.2 \\
-0.3 \\
+0.1 \\
+0.2 \\
+0.1 \\
+0.4 \\
-0.1 \\
-0.3 \\
-0.4 \\
-0.1\end{array}$ \\
\hline
\end{tabular}

they leave the following differences between the computed and observed values:-

'The differences may be considered within the uncertainty of the observations.

The annexed diagram shows the comparison given above:-

Observed and computed half-monthly inequality in height from observations. of the ligh waters. of the low waters.

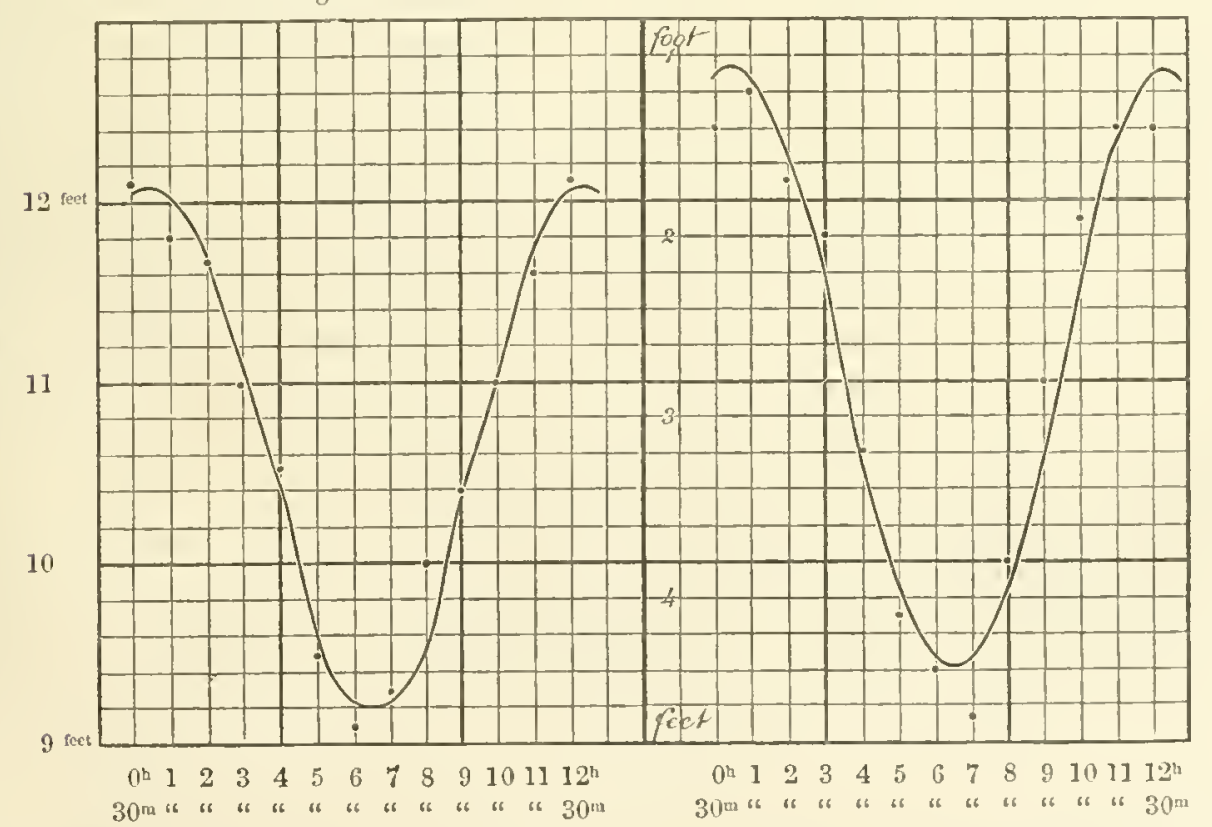

From the inequality in height $\frac{h}{h^{\prime}}$ or $\frac{S^{\prime \prime \prime}}{I^{\prime \prime \prime}}$ (notation of the wave theory) $=0.36$ 个 whereas from the inequality in times $\frac{h}{h^{\prime}}$ or $\frac{S^{\prime \prime}}{M^{\prime \prime}} \cdot \quad \cdot \quad \cdot \quad \cdot \quad=0.471$ 
The ratio ${ }^{1}$ of the solar to the lunar tide is deduced with more exactness from the inequality in times, and the above value is certainly greater than the average value deduced at more sonthern stations. One of the reasons why this ratio is not constant, and which probably applies here, is giren in $(538, \beta)$ (Tides and Waves), viz.: If ticles are commmnicated by different cliamels to the same port, the proportion of the solar and lumar waves will depend on the length of those channels. This explanation would require a polar tide to enter throngl Kennedy Channel, to combine with the principal tide which passes up Baffin's Bay, and enters by Smith's Straits. According to the equilibrium theory, there should be no tide at the pole, and but a small tide in latitude $782^{\circ}$; but it is the tidle wave propagated from the Atlantic, which is felt in this part of the polar regions. With regard to $\alpha$, its value as found by the heights is more accurate than that found by the times; the latter gave $\alpha=9^{\circ}$, the former $15^{\circ}$ (the same from high and low waters). Adopting $15^{\circ}$, the retard or age of the tide becomes 1 lay, by which interval the spring and neap tides follow the syzygies and quadratures, respectively. The timevalue of $\alpha$ is here smaller than the height-value, which is more in accordance with theory than the opposite, as observed at a number of places on the coast of England (543 and 546, Tides and Wares). Compared with other ralues of $\alpha$, the Van Rensselaer value appears somewhat smaller than an arerage at more southem stations.

We have further, mean rise and fall of tides at Van Rensselaer Bay 7.9 feet, range of spring tides 11.1 feet, and range of neap tides 4.7 feet. These numbers are arerages from the discussions of $9 \frac{1}{2}$ lunations, and obtain withont regard to the diumal inequality, which will be investigated further on.

Effect of the Changes in the Moon's Declination and Parallax on the half-monthly Inequality, in Time.-In reference to the investigation of the half-montluly inequality, it is comparatively of little consequence which transit of the moon is taken for comparison; it is otherwise in the investigation of the effect of a change in the moon's declination and parallax, as well as for a similar effect dne to the sun, which latter, howerer, cannot become a subject of investigation for the tidal series in hand, on account of its short extent; for the same reason, the variation in the inequality, in height, will have to be passed orer. To ascertain the effect due to the moon's declination and parallax, an anterior value, corresponding to a certain age of the ticle, is to be taken in the comparison; the preceding investigation gave for the retard 1 d day, each lunitidal interval, minus its corresponding mean value for the respective hour of the moon's transit, was therefore tabulated in respect to the moon's declination and parallax (separately for each), corresponding to one day anterior to the time of high or low water, thus referring the results to transit $E$. The present investigation can only furnish an approximation to the true results; the

\footnotetext{
"For comparison of different values for this ratio, the following have becu selected: $\frac{S^{\prime \prime}}{W}$ for London, 0.37 ; for Plymontlu, 0.107 ; from the discussions of the Superintentent of the U. S. Coast Survey, for

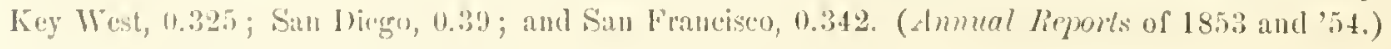

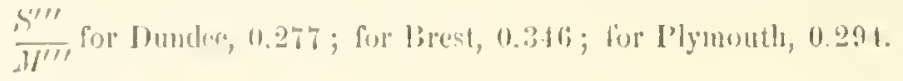


observations, while they give reliable value for the half-montlily inequality, cannot be expected to give more than an approximation to its variations. For any one station, and any one inequality or correction to it, special examinations require to be made to ascertain that transit of the moon, best suited for the purpose; this has hardly been done for any standard station, and it snffiecs to state liere that, by referring to an anterior transit, the whole half-montlyly inequality is mored backward through nearly twenty-four minutes for every transit preceding. Upon the inequality itself, the effect is bit of a differential chantacter. Thus to refer our table to transit $E$, deduct $24^{\text {m }}$ from each value.

'To concentrate as many values as possible to a mean, the changes of declination and parallax were grouped for three values. The separnte parcels for declination are for declination 0 to $13^{\circ}, 13^{\circ}$ to $21^{\circ}$, and $21^{\circ}$ to $27^{\circ} .5$, irrespective of sign. The parallax gromps are: $5 t^{\prime}$ to $56^{\prime}, 56^{\prime}$ to $58^{\prime}$, and $58^{\prime}$ to $61^{\prime} .4$.

The differences of interval for the high and low watcrs were made out separately, and, in general, agreed tolerably well. 1 obtained the following results:-

TABLE SHOWING TIE CORRECTION (IN MINUTES) TO TUE MEAN IIOURLY INTERVAL, FOR A CIINGE IN TIE MOON'S DEClasation AND PARALLAX.

\begin{tabular}{|c|c|c|c|c|c|c|}
\hline \multirow[b]{2}{*}{ Moon's transit. } & \multicolumn{3}{|c|}{ Corrcclion to interral for moon's declination. } & \multicolumn{3}{|c|}{ Correction to interval for moon's parallax. } \\
\hline & $0^{\circ}$ to $13^{\circ}$ & $13^{\supset}$ to $21^{\circ}$ & $21^{\circ}$ to $27^{\circ} .5$ & $54^{\prime}$ to $56^{\prime}$ & $56^{\prime}$ to $58^{\prime}$ & $5 S^{\prime}$ to $61^{\prime} .4$ \\
\hline $\begin{array}{cc}0^{\mathrm{h}} & 30 \mathrm{~m} \\
1 & 30 \\
2 & 30 \\
3 & 30 \\
4 & 30 \\
5 & 30 \\
6 & 30 \\
7 & 30 \\
6 & 30 \\
9 & 30 \\
10 & 30 \\
11 & 30\end{array}$ & $\begin{array}{l}-2{ }^{\mathrm{m}} \\
+15 \\
+21 \\
+23 \\
+8 \\
+14 \\
-8 \\
+1 \\
-2 \\
-6 \\
+1 \\
-3\end{array}$ & $\begin{array}{l}-7 \\
-5 \\
-7 \\
-11 \\
0 \\
+4 \\
\pm 15 \\
\pm 13 \\
+9 \\
+1 \\
-6 \\
-2\end{array}$ & $\begin{array}{l}+5^{\mathrm{m}} \\
-18 \\
-12 \\
-8 \\
-13 \\
-15 \\
-3 \\
+9 \\
+15 \\
+22 \\
+10 \\
+3\end{array}$ & $\begin{array}{l}-12 \mathrm{~m} \\
-17 \\
-11 \\
-1 \\
-1 \\
+16 \\
+7 \\
+5 \\
+26 \\
+2 \\
+10 \\
-4\end{array}$ & $\begin{array}{l}+14 \\
+17 \\
+12 \\
+20 \\
+1 \\
+19 \\
-3 \\
-3 \\
-10 \\
-11 \\
-9 \\
+4\end{array}$ & $\begin{array}{l}-7 \\
+5 \\
-2 \\
-10 \\
-3 \\
-36 \\
-2 \\
-6 \\
+8 \\
-5 \\
+7 \\
+4\end{array}$ \\
\hline Mean & $+5^{11}$ & $-1^{\mathrm{ma}}$ & 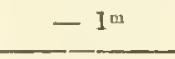 & $+2^{m}$ & $+4^{m}$ & -503 \\
\hline No. of obserF. & 373 & 262 & $34 \varsigma$ & 387 & 244 & 333 \\
\hline
\end{tabular}

Mean declination $16^{\circ}, 0$.

Hean parallas 57 '.

The above table of declination corrections exhibits systematic values for the periodical part of the lunar effect, or for the term $D \sin .2(\phi-\gamma)$. Between $0^{\circ}$ and $13^{\circ}$ of declination, the correction is positive for transits between $1^{\mathrm{h}}$ and $7^{\mathrm{h}}$, for other hours negative; for declinations between $13^{\circ}$ and $21^{\circ}$ it is positive, between the hours of 4 and 10 ; for remaining hours it is negative, and for declinations $21^{\circ}$ to $2 \tau^{\circ} .5$, the correction is positive, for hours $i$ to 1 , and negative for remaining hours of transit. The quantity $D$ is accordingly about 14 minutes, and $\gamma$ cquals $15^{\circ}, 60^{\circ}$, and $105^{\circ}$ respectively.

'The variation in the inequality lue to the changes of the moon's declination appears large when compared with its value at other places, but is in conformity with the large value of the half-monthly inequality itself.

The periodical part of the parallax correction is of the same form as given above. 
The empirical values for the groups of small and middle values of parallax appear systematic; the ralues in the last column for large parallax are less regular. 'The maximum correction on the average is somewhat greater than one-fourth of an hour.

The corrections to the mean establishment for changes of the sun's declination and parallax may be taken as one-third of the corresponding lunar values, and in the present ease will probably not exceed five minutes of time.

The means of each column, containing the non-poriodical part, are small, and appear rather irregular; they are variable with the transit or the moon's age adopted in the discussion.

Diumal Inequality. - Wre now proceed to the examination of a prominent feature in the Rensselaer Harbor tides, namely, the dimmal inequality. This inequality is well marked in the diagrams, Plates I, II, and III. Although the existence of this inequality, in leight and times, has long been linown to practical men, it was not until abont twenty-five years ago that its laws were understood and reduced to computation by Mr. Whewell. ${ }^{2}$ 'The subject has since been taken up by the present superintendent of the U. S. Coast Survey, Prof. Bache; ${ }^{3}$ his researehes commenecd about nine years ago, and resulted in a further extension of the method of discussion as well as in the recognition of the geographical limits of the phenomena on our own coast; further, the discussion of single day tides, produced by this inequality in extreme eases, and here complicated by an extremely small rise and fall of the tides, was now successfully accomplished. According to the equilibrim theory, the diumal tide ought to be very small in latitude $79^{\circ}$; but riewing the Rensselaer Harbor tide as a ware, produced principally in the Atlantic, and propagated throngh Daris's and Smith's Straits, the existence of the diurnal inequality in so high a northem latitude camnot surprise us. 'The following notes were extracted from Captain McClintock's narrative of the voyage of the "Fox,"

1 On this point the reader may consult Whewell's 9th series of tidal researches: "Laws of the Tides from a Short Series of Observations," I'hil. 'Trans. 1838; also Airy, "Tides and Wares," articles 552 and following.

2 Researches on the Tides, sixth series. On the Results of an Extensire System of Tide Ohserrations male on the Coasts of Europe and America in June, 1835. By the liev. W. Whewell. Phil. Trans. lioy. Sue. 1836.

fiesearches un the Tides, serenth scries. On the Dimmal Inequality of the IIeight of the Tide, especially at I'lymouth and Singalure. Jy the same author. l'hil. Thans. 1837.

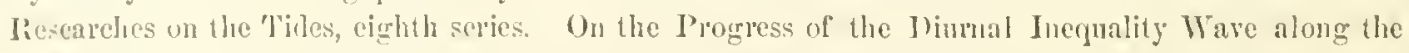
C'oastis of Europe. liy the same anthor. Ihil. Trans. Roy. Suc. 183\%.

a Xote or a lliscussion of Tirlal Observations at Cat Istant iu the Gulf of Alexieo, hy Irof. A. D. bache. Const Survey Iiejurt for $1851, \Lambda \mathrm{p}$. No. $\tau$; Adlitional Notes thereto, Coast Survey lieport for 1852, А|l. Nu. 22.

On llo Thites at Key Wrest and of the Westem Const of the United States. Coast Survey Ireport for 185\%, All). Yor. 27 and 23. 13y J'rof. A. I). Bache.

(i)mprarisun of the Itimal Inerpality of the Tides at San Diego, San Franciseo, and Astoria, on the

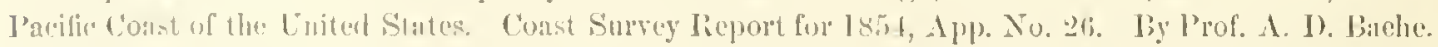

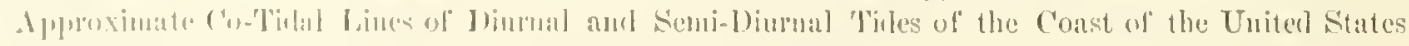

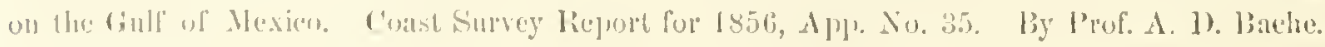

For the theoreljeal investigntion of the diurat tide, sece also $A$ iry's flides and Waves, artictes 46 and follewing ; and artictes itt2 and lollowing. 


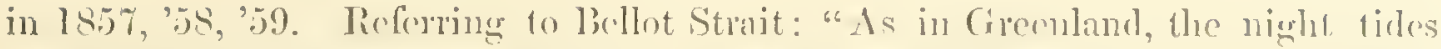
are much higher than the diry tides." Spenking of the ice motion, ant remarliner that the tides are the chicf canse of it, he salys: "Now we know that the night tides in Greenland greatly exceed the day tides." Also, when near linchan Island, north of Upernarik, and in the vicinity of Cape Shackleton: "Te had gromnded during the day tide, and were floaterl off by the night tide, which on this coast occasions a much greater rise and fall." Jy the labors of 1)r. Kane we now know that the dimmal inequality extends as high up as $79^{\circ}$ of latiturle on the northwestern coast of Upper Greenlind. In a report of Mr. Somntag's to 1)r. Kiane, dated Godharn, Sept. 12, 1855, he says: The mean height of spring tides is 12.8 feet, and at the time of new and full moon high water is at $12^{\mathrm{h}}()^{\mathrm{m}}$; the highest spring tide is three days after full moon, and the night tide is at this time fully three feet ligher than the diny tide. It Northumberland Island, Scpt. 10, 1854, at (after) the time of full moon high water was at $11^{\mathrm{h}} \mathrm{P}$. M., and the night tide rose three feet more than the day tirle. These statements, erucle as they necessarily are, show that the attention of the party was fully directed to the phenomenon.

A cursory examination of the I'lates (I, II, and IlI) shows that the diurnal inequality extends without exception over the whole series of observation, that it is well marked in the difference of the height of high witer. but rery little or irregularly in the lieight of low wister; that sometimes the cliy tide, at other times the night tide is the higher of the two occurring in a lunar day; further, that it vanishes a day or two after the moon's crossing the equator, and that it amounts in maximo to about three fect some time after the moon attains les greatest declination. There is but one instance where the inequality ipproximates to the production of a single day ticle. See curve for Nov. 23, 18.5 .3$.

We may now enter somewhat more fully into the discussion of this inequality, which is produced by the interference of two independent wares, the dimmal and the semi-diurnal, the former depenting for its size chiefly on the moon's declination. For a complete study of these compound waves, they require to be examined in their separate parts, and it would therefore be our first object to effect their separation into the diurmal and the semi-diurnal; a process which, when graphically performed, is neither too laborious nor lacking in accuracy; it is nevertheless a process of some nicety, and requires observations of standard excellence. Upon trial, I found the less rigorous method employed by Mr. Whewell in his discussion of the Plymouth and Singapore tides, was better suited to the general mass of the observations at Van Rensselacr, and that the above described process of separation had better be rescrved to that portion of our observations which are apparenty of the best character.

The observed heights of high and low water were laid down graphically, and a line was drawn by the eye, cutting off the zigzags of the successive high waters, leaving equal portions above and below the intermediate curve. These differenees from the mean height were then set off from another axis, and those belonging to the high water next following the moon's superior transit were markerl by a curve of dashes; those following the moon's inferior transit were marked by a curre of dots. These curves, without exception, were found to lave alternately, as the 
moon has north or south declination, positive and negative ordinates, in perfect accordance with the equilibrium theory, according to which the tide (high water) which belongs to a south transit of the moon should be the greater of the two of the same day, the moon's declination being north, or should be the smaller of the two, the moon haring sonth declination; when the moon crosscs the equator (or, according to experience, some time after it), the incquality vanishes; the time by which the full effect is produced is, as in other cases of the application of this theory, later than theoretically indicated. On Plite III are given specimens of the diurnal inequality curre, construeted as explained above and on the same seale as the other diagrams on these plates. By means of the diagrams, the epoch when the inequality ranishes has been made ont as follows:-

T.IBLE SHOWING THE OBSERIED TIMES WIEN THE DLURAL INEQUALITY YANISHES, TOGETHER WITH THE TINE WHEN THE MOON CROSSES THE EQUATOR, AND TIE DIFFERENCE OF THESE TIMES, OR THE NUMBER OF DAYS BY WIICU TIE CAUSE PRECEDES THE EFFECT. TIIS DIFFEREXCE IS ALSO CALLED THE EPOCH.

\begin{tabular}{|c|c|c|c|c|c|c|c|}
\hline Tear. & $\begin{array}{l}\text { Inequality } \\
\text { disappears. }\end{array}$ & $\begin{array}{l}\text { Inon's decli- } \\
\text { nation equal } 0 .\end{array}$ & $\begin{array}{l}\text { Difference, } \\
\text { or eyoch. }\end{array}$ & lear. & $\begin{array}{l}\text { Inequality } \\
\text { disnppears. }\end{array}$ & $\begin{array}{l}\text { Monn's decli- } \\
\text { nation equal } 0 .\end{array}$ & $\begin{array}{l}\text { Difference, } \\
\text { or epoch. }\end{array}$ \\
\hline \multirow{2}{*}{$\begin{array}{c}1, .53 \\
6\end{array}$} & \multirow{2}{*}{$\begin{array}{l}\text { Oct. not observerl } \\
\text { Oct. } 30^{3} 21^{\mathrm{h}}\end{array}$} & $1.5^{d} \quad 7^{h}$ & $\ldots$ & \multirow{2}{*}{ 195. } & April 2:i हi & $24^{\mathrm{a}} \quad 11^{\mathrm{h}}$ & $3^{4} 21^{\mathrm{h}}$ \\
\hline & & $29 \quad 15$ & $1^{d} \quad 3^{n}$ & & Hay 924 & 911 & 10 \\
\hline$a$ & Nin. 12 101 & $11 \quad 13$ & & : & $\begin{array}{lll}* & 23 & 14\end{array}$ & $21 \quad 17$ & 121 \\
\hline & Wnr. 13 ij & & & 64 & Inne $7 \quad 9$ & $5 \quad 10$ & 23 \\
\hline 16 & $\begin{array}{lll} & 27 & 22\end{array}$ & 25 & 1 IS & $\because$ & “ 19 & $17 \quad 22$ & 11 \\
\hline$\because$ & Dec. 9 & 19 & (1) 14 & 6 & July 5 & 217 & 11 \\
\hline ، & $\begin{array}{lll}\text { "6 } & 25 & 12\end{array}$ & 13 & 123 & “ & $\begin{array}{lll} & 31 & 22\end{array}$ & $29 \quad 22$ & 2 \\
\hline $1=54$ & Jan. not observerl. & 2 & - - & $" 6$ & Sept. 93 & 722 & 1 \\
\hline " & " 6 6 6 & $19 \quad 18$ & $\cdots$ & & Resnaining observa- & 229 & $\cdots$ \\
\hline is & lieb. 34 & 10 & $1 \quad 18$ & & tions of $S$ ries $1 V$ & & $1 \mathrm{~d} 15 \mathrm{~h}$ \\
\hline$"$. & $\begin{array}{lll} & 16 & 18 \\
3 & 19\end{array}$ & 23 & $\begin{array}{ll}0 & 19\end{array}$ & & liable. & Mean. & 1010 \\
\hline " & Mar. 112$\}$ & 19 & 117 & & & & \\
\hline$" 4$ & " $16^{\circ}, 0$ & 15 & ก 19 & & & & \\
\hline 66 & Mar. obs'n inconple. & 20 & $\cdots$ & & & & \\
\hline
\end{tabular}

The results for the cpoch are rery regular, and with the exception of part of the last serics, which is of inferior accuracy, no observation has been omitted. The inequality ranishes at the distance of 1.62 days' motion of the moon from her nodes.

'The magnitude of the diurnal inequality, and its rariation depending on twice the moon's declination, was made out by dividing the inequality curves in six parts between the times of disappearance, and by tabulating the ordinates as well as the corresponding declination of the moon, the following results were obtained from 12 complete eycles, omitting no value, viz:-

AMUUNT OF DIURNAL INEQUALITY IN THE IIEIGHT OF HIGIL WATER.

\begin{tabular}{|c|c|c|c|c|c|c|c|c|c|c|c|c|c|c|}
\hline Oralinate. & & & & & & (In & et.) & & & & & & Mean & Menn \\
\hline 11 & 11 & 11 & 1) & 0 & () & $n$ & (1) & () & 0 & 0 & (1) & 0 & 0.0 & $0^{\circ}$ \\
\hline I & 1.1 & 8.1 & 11.8 & 2.7 & 1.0 & 0.3 & 0.2 & 1.4 & 2.7 & 1.5 & 1.5 & 1.01 & 1.4 & 12 \\
\hline 2 & 01.5 & 2.9 & 2.33 & 4.0 & 1.21 & 2.5 & 1.11 & 1.5 & 2.2 & 2.11 & 2.1 & 3.0 & 2.3 & 21 \\
\hline 3 & 1.5 & 2.2 & 3.1 & 1.6 & 4.0 & 2.6 & 1.1 & 1.6 & 2.2 & 3.1 & 2.0 & 2.0 & 2.5 & 25 \\
\hline 1 & 1.6) & 2.3 & $\therefore .1$ & $4.1 i$ & 0.2 & $3.1 \%$ & 1.5 & 1.1 & 1.4 & 1.8 & 3.3 & 2.7 & 2.3 & 203 \\
\hline 5 & 11.8 & 1.1 & 11 & 3.0 & 2.4 & 1.2 & 0.7 & 3.5 & 2.2 & 1.0 & 2.0 & 2.11 & 1.7 & 13 \\
\hline 6 & 11 & 11 & 1) & 0 & 0 & v & 0 & 0 & 0 & 0 & 1 & 0 & 0.0 & 0 \\
\hline
\end{tabular}


The mean declination corresponds to an epoch 1.6 days anterior, which remark applies also to the formula $d h=C \sin .2 \delta$, representing the diurnal inequality $d h$ in two sticcessive high or low waters, $\delta$ ' being the moon's declination. For the value of $C$ we obtain 3.3, which gives us the following comparison:-

DIURNAL INEQUALITY IN IIFIGIIT.

(Epoch 1.6 days.)

\begin{tabular}{|c|c|c|c|}
\hline Moon's declination. & Obserred $d h$. & Computed $t h$. & Difference. \\
\hline $\begin{array}{c}00 \\
12 \\
21 \\
25 \\
22 \\
13 \\
0\end{array}$ & $\begin{array}{c}\text { Feet. } \\
11.11 \\
1.4 \\
2.3 \\
2.5 \\
2.3 \\
1.7 \\
0.0\end{array}$ & $\begin{array}{c}\text { Feet. } \\
0.0 \\
1.4 \\
2.2 \\
2.5 \\
2.3 \\
1.5 \\
0.0\end{array}$ & $\begin{array}{c}\text { Feet. } \\
11.11 \\
0.0 \\
0.1 \\
0.0 \\
0.0 \\
0.2 \\
0.0\end{array}$ \\
\hline
\end{tabular}

The diurnal inequality in time I have tried to exhibit by numbers as well as by diagrams; it seems, however, that the incidental irreguluritics in the observations themselves, coupled with the fact that the observations generally were only made half-hourly and at other times lourly-so far exced in magnitude the inequality itself as to make the effect of the changes of the moon's declination exceedingly obscure. The luniticlal intervals (for high and low water) between Oct. 17 and Dec. 28, 1853, between Jan. '28 and March 7, 185t, and between June 1 and July 7,1854 , were tabulated in vertical columns; the means of the altemate values were tabulated in the $2 \mathrm{~d}$ column, and placed in the horizontal line opposite the intermediate valne of column one. The numbers in the first colnm were next subtracted from the corresponding numbers in the second column, if the interval belonged to the inferior transit; if belonging to the superior, the ralnes in the second column were subtracted from those in the first. The moon's declination, for $n$ oon each day, was also set down. The 276 values for diumal inequality in time, thus obtained, were plotted. After attempting to deduce an epoch and arranging the values for different assumptions for epoch, no satisfactory result could be obtained in any way according with the expression

$$
d \psi=\frac{g \tan . \delta^{\prime}}{1+A \cos ^{2} \phi}(\text { see Lubbock, Phil. Trans. 183\%), }
$$

and the results of the investigation must be confined to the following general remark. The dimmal inequality in time is in maxima probably not exceeding two hours; it seems to be less in amount for the times of high water than for the times of low water, a result the reverse of that belonging to the inequality in height. A similar conclusion was arrived at in the discussion of the tides at San Francisco, Cal. ('Prof. A. D. Bache in Coast Survey Report for 1853, p. *81), when the smaller ineqnality in height of high water (when compared with that for low water) corresponded to the greater inequality in time of high water (when compared with the inequality for low water). Whether the inequality of the height for high or low water is the greater or smaller depends only on the epoch of the dimrnal wave compared with the epoch of the scmi-dimmal ware. There is no regular increase 
of the inequality corresponding to an increasing (irrespective of signn) declination of the moon, but the curve appears double-crested about the time of maximum declination, there being a sudden diminution in the inequality, preceded and followed by lighl values; about the time of the moon's crossing the equator the inequality is rery irregular.

On Plate IV, the actual separation of the semi-diumal and the dimmal wave has been effected graphically, for which purpose a part of the best observations was selected; these observations extend over the period from Oct. 30 to Nov. 22, 1853. The process of decomposition in use in the U.S. Coast Survey was at first an analytical one, by computing sine curves; since 1555 , however, a graphical process, equiralent thereto, was substituted; this latter method, as introduced by assistant L. F. Pourtales, may be briefly explained as follows: After the observations are plotted and a tracing is taken, the traced curves are shifted in epoch 12 (lunar) hours forward, when a mean curve is pricked off between the observed and traced curves; this process is repeated after the tracing paper has been shifted 12 hours backward; the average or mean pricked curve thus obtaincd represents the semidiumal wave. On an axis parallel with that on which the time is counted, the differences between the originally observed and the constructed semi-dimnal wave were laid off; this constitutes the dinmal curve. In the case in hand I have simplified the process of sepraration by blackening the under surface of the tracing paper with a lead pencil, and rmming in with a free hand; the intermediate curves by the pressure of a style, an arerage of the two traces thus left on the lower paper, gave the semi-dinmal wave in quite an expeditious manner. On the diagram, the diurnal curve with its epoch of high water nearly coinciding with that of the semidiunal wave, appears plainly with its variation in size depending on the moon's declination.

Investigation of the Form of the Tide WTave.-The shape of the tide wave has been ascertained in the manner described in art. (479) 'Tides and W'ares, and depends on the honly observations of 60 tides, 30 dnring spring tides and an cqual number during neap tides, that is, the observed heights on the day of the sycygies and quadratures and on the first and second day after, were tabulated, forming ten groups of three columns each, from low water to low water. The cohmms of an equal number of hours (they vary from 16 hours to 11 hours) were united in a mean. In order to combine these it was assumed that the interval from the observed low water to the next following low water corresponds to $360^{\circ}$ of phase, and the time of erery intermediate obscration was converted into pluase by that proportion. In order to render the observed heights eomparable, the range from high to low water in every half tide (the reading of low water for phase o generally not being identical with the reading of the succecling low water or phase $3\left(60^{\circ}\right)$ was supposed to correspond to 2.00, and the olevition abore the low water was converted into number by that proportion, thus furnishing a scrics of ordinates for equidistant aluscissa. 'T'he means of all the phases and corresponding converted depressions within erery 30 th degree of phase were then taken with proper regard to the weights, depcuding on the number of colnmms, of cqual hours, nnited at the commencement of the reduction. By observation of the prougress of the numbers, 
it was easy to alter the latter so as to make them exactly correspond to the phases $30^{\circ}, 60^{\circ}, 90^{\circ}, 120^{\circ}$, etc. In this manner the following numbers lave been obtained :-

FOR TIE SPRING-TIDE WAYE OCCURIING ONE AND $\Lambda$ QUARTER DAY AFTER FULL AND NEW MIDON.

\begin{tabular}{|c|c|c|c|c|c|c|c|c|c|c|c|c|c|}
\hline \multicolumn{7}{|c|}{ Phase of groups. } & \multicolumn{7}{|c|}{ Proportional height above low water. } \\
\hline & $\begin{array}{c}00^{\circ} \\
26 \\
51 \\
77 \\
103 \\
129 \\
154 \\
180 \\
206 \\
231 \\
257 \\
283 \\
309 \\
334 \\
360\end{array}$ & $\begin{array}{c}10^{\circ} \\
25 \\
55 \\
83 \\
111 \\
138 \\
166 \\
194 \\
222 \\
249 \\
277 \\
305 \\
332 \\
360\end{array}$ & $\begin{array}{r}0^{\circ} \\
30 \\
60 \\
90 \\
120 \\
150 \\
150 \\
210 \\
240 \\
270 \\
300 \\
330 \\
360\end{array}$ & $\begin{array}{c}0^{\circ} \\
33 \\
65 \\
95 \\
131 \\
16 i 4 \\
196 \\
229 \\
262 \\
294 \\
327 \\
360\end{array}$ & $\begin{array}{c}0^{\circ} \\
36 \\
72 \\
118 \\
144 \\
140 \\
216 \\
252 \\
246 \\
324 \\
360\end{array}$ & $\begin{array}{c}\text { Mean. } \\
11^{\circ} \\
30 \\
59 \\
59 \\
120 \\
156 \\
140 \\
208 \\
237 \\
270 \\
300 \\
330 \\
360\end{array}$ & & $\begin{array}{l}0.00 \\
0.116 \\
0.32 \\
0.01 \\
1.39 \\
1.81 \\
1.94 \\
2.00 \\
1.84 \\
1.58 \\
1.14 \\
0.60 \\
0.15 \\
0.02 \\
0.00\end{array}$ & $\begin{array}{l}0.00 \\
0.23 \\
0.65 \\
1.13 \\
1.68 \\
2.04 \\
1.98 \\
2.100 \\
1.84 \\
1.23 \\
0.79 \\
0.40 \\
0.110 \\
0.110\end{array}$ & $\begin{array}{l}0.00 \\
0.24 \\
0.90 \\
1.36 \\
1.73 \\
1.90 \\
2.011 \\
1.84 \\
1.45 \\
1.00 \\
0.27 \\
0.17 \\
0.00\end{array}$ & $\begin{array}{l}0.010 \\
0.27 \\
0.701 \\
1.32 \\
1.76 \\
1.93 \\
2.00 \\
1.56 \\
1.15 \\
0.05 \\
0.25 \\
0.00\end{array}$ & $\begin{array}{l}0.00 \\
0.10 \\
0.46 \\
1.17 \\
1.52 \\
2.00 \\
1.48 \\
1.23 \\
0.70 \\
0.41 \\
0.00\end{array}$ & $\begin{array}{c}\text { Mean. } \\
0.00 \\
0.21 \\
0.71 \\
1.24 \\
1.70 \\
1.95 \\
2.011 \\
1.88 \\
1.46 \\
0.97 \\
0.37 \\
0.17 \\
0.00\end{array}$ \\
\hline Weight & 5 & 4 & 13 & 7 & 1 & & Weight & 5 & 4 & 13 & 7 & 1 & \\
\hline
\end{tabular}

The columns header "mearı" show the ordinates of the wares for (nearly") equidistant intervals of time.

'The following table contains the corresponding numbers for the neap tirle wave occurring 11 day after the first and last quarter, and as derived from 30 tides observed hourly from low to low water:-

\begin{tabular}{|c|c|c|c|c|c|c|c|c|c|c|c|c|c|c|c|}
\hline \multicolumn{8}{|c|}{ Phase of groups. } & \multicolumn{8}{|c|}{ Proportional height abore low water. } \\
\hline & $\begin{array}{r}0^{\circ} \\
24 \\
48 \\
72 \\
96 \\
120 \\
144 \\
165 \\
192 \\
216 \\
240 \\
264 \\
248 \\
312 \\
336 \\
260\end{array}$ & $\begin{array}{c}0^{2} \\
26 \\
51 \\
76 \\
103 \\
129 \\
154 \\
180 \\
206 \\
231 \\
257 \\
283 \\
309 \\
334 \\
360\end{array}$ & $\begin{array}{r}0^{\circ} \\
25 \\
55 \\
83 \\
111 \\
135 \\
166 \\
194 \\
222 \\
249 \\
277 \\
3105 \\
332 \\
360\end{array}$ & $\begin{array}{c}00 \\
30 \\
60 \\
90 \\
120 \\
150 \\
150 \\
210 \\
240 \\
2711 \\
300 \\
330 \\
360\end{array}$ & $\begin{array}{c}0 \circ \\
33 \\
65 \\
98 \\
131 \\
164 \\
196 \\
229 \\
262 \\
294 \\
327 \\
360\end{array}$ & $\begin{array}{r}0^{\circ} \\
36 \\
72 \\
108 \\
144 \\
1 \backsim 0 \\
210 \\
252 \\
288 \\
324 \\
360\end{array}$ & $\begin{array}{c}\text { Mean. } \\
\\
y^{\prime} \\
29 \\
54 \\
89 \\
119 \\
147 \\
180 \\
213 \\
241 \\
271 \\
3011 \\
331 \\
360\end{array}$ & & $\begin{array}{l}0.00 \\
0.07 \\
0.26 \\
0.49 \\
0.89 \\
1.19 \\
1.55 \\
0.82 \\
2.00 \\
1.02 \\
1.151 \\
1.35 \\
0.94 \\
0.51 \\
0.23 \\
0.00\end{array}$ & $\begin{array}{l}0.00 \\
0.17 \\
0.55 \\
1.11 \\
1.53 \\
1.53 \\
2.00 \\
1.95 \\
1.62 \\
1.43 \\
1.09 \\
0.67 \\
1.24 \\
0.105 \\
0.00\end{array}$ & $\begin{array}{l}0.00 \\
0.24 \\
0.59 \\
0.93 \\
1.35 \\
1.73 \\
2.10 \\
1.96 \\
1.72 \\
1.32 \\
11.601 \\
0.32 \\
0.04 \\
10.00\end{array}$ & $\begin{array}{l}0.00 \\
0.20 \\
0.50 \\
1.115 \\
1.60 \\
1.65 \\
2.00 \\
2.00 \\
1.44 \\
1.37 \\
0.79 \\
0.42 \\
0.00\end{array}$ & $\begin{array}{l}0.00 \\
10.60 \\
1.01 \\
1.41 \\
1.81 \\
2.00 \\
1.72 \\
1.43 \\
0.79 \\
0.15 \\
0.016 \\
0.00\end{array}$ & $\begin{array}{l}0.00 \\
1.00 \\
0.35 \\
1.06 \\
1.65 \\
2.00 \\
1.63 \\
0.99 \\
0.10 \\
0.019 \\
0.00\end{array}$ & $\begin{array}{l}0.00 \\
0.20 \\
0.52 \\
1.08 \\
1.51 \\
1.52 \\
1.97 \\
1.44 \\
1.58 \\
1.08 \\
0.56 \\
11.22 \\
0.00\end{array}$ \\
\hline Weight & 3 & 4 & 8 & 13 & 1 & 1 & & Weight & 3 & 4 & 8 & 13 & 1 & 1 & \\
\hline
\end{tabular}

The results are representer in the annexcl diagram. The result for the neap ticte curve has also been multiplied by $\frac{4.7}{11.1}$, the ratio of neap and spring ticle range as found on a preceding page, and was increased by 0.5 to refer it to the same level. 


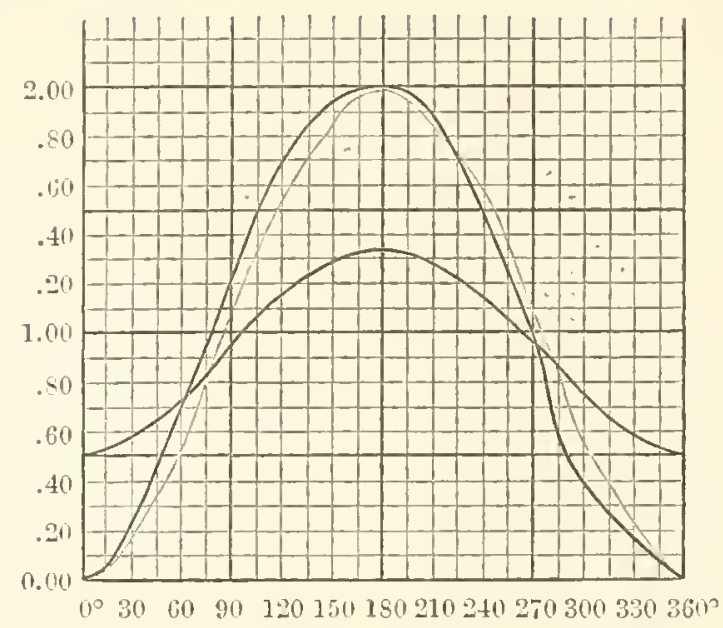

The full curves in the diagram show the form of the spring and neap tide wave (the scales being arbitrary), to which has been added for convenient comparison the dotted curve representing the neap tide wave on the same relative scale as the spring tide wave. It is apparent that the spring tide wave is slightly steeper between low and high water than between high and low water, and that the neap tide wave is very nearly symmetrical in respect to rise and fall.

We have seen that the duration of rise is $6^{\mathrm{h}} 04^{\mathrm{m}} .7$, hence the duration of fall will be $6^{\mathrm{h}} 19{ }^{\mathrm{m}} \mathrm{r}$; or in making ebb the time is 15 minutes greater than in making flood, a circumstance in conformity with the shape of the curres of rise and fall. This holds good for an average tide; according to art. (510) Tides and Waves, if the place of observation is not far from the sea, or, as in our case, in a bay, the water will occupy a shorter time to rise than to fall, and the incquality will be greater at spring tides than at neap tides; this is fully illustrated in the preceding diagram, the spring ticle wave being the stceper of the two.

The form of the tide waves will be found closely represented by the following expressions:-

For the spring tide wave-

$$
5.83+5.58 \sin \cdot\left(\theta+278^{\circ}\right)+0.20 \sin \cdot\left(20+281^{\circ}\right)
$$

For the neap tide ware-

$$
2.42+2.25 \sin .\left(0+269^{\circ}\right)+0.09 \sin .\left(20+290^{\circ}\right) ;
$$

in which expressions the angle $\theta$ counts from low water to low water, from 0 to $360^{\circ}$, and the height of the wave is expressed in fect.

The relative numbers, griven above, as the ordinates, have been changed in the proportion of 2 to 11.1 for the higher and of 2 to 4.7 for the lower wave. The following table shows the agreenent between observation and the numerical expressions, in which the :3l and higher terms are zero:- 
FORA OF THE TIDE WAYE AT YAN RENSSELAER IHARBOR.

\begin{tabular}{|c|c|c|c|c|}
\hline \multirow[b]{2}{*}{ Phase. } & \multicolumn{2}{|c|}{ Ileight of Spring tide, in feet. } & \multicolumn{2}{|c|}{ Ileight of neap tide, in feet. } \\
\hline & Observed. & Computed. & Observed. & Computed. \\
\hline 0 & 0.0 & 0.1 & 0.0 & 0.1 \\
\hline 30 & 1.2 & 1.4 & (0.5) & 0.4 \\
\hline 60 & 3.9 & 3.9 & 1.3 & 1.3 \\
\hline 90 & 15.9 & 10. & 2.5 & 2.5 \\
\hline 120 & 9.4 & 9.3 & 3.5 & 3.5 \\
\hline 150 & 111.7 & 111.9 & 4.3 & 4.3 \\
\hline 150 & 11.1 & 11.1 & $4.1 ;$ & 4.5 \\
\hline 210 & $111 .-4$ & 10.2 & 4.3 & 4.4 \\
\hline 240 & 7.9 & 8.11 & 3.7 & $3 . \overline{7}$ \\
\hline 270 & 5.4 & 5.3 & 3.5 & 2.5 \\
\hline 300 & 2.1 & 2.4 & 1.3 & 1.3 \\
\hline 330 & 11.9 & 11.5 & 11.5 & 0.4 \\
\hline 360 & 11.11 & 11.1 & 11.11 & 0.1 \\
\hline
\end{tabular}

Respecting the effect of the wind and ice on the tides, it may be remarked that the former can only be slight, since the sea is protected from the direct action of the wind by its icy cover for the greater part of the year. When the sea is partially open, the effeet becomes sensible, as may be seen by the following note extracted from the log-book:-

"Augnst 17, 1853. The above records show a heary gale from the southward gradually hauling to the eastward; the eftect of this gale on the tides was very marked; our flood rose two feet above any previous register, overflowing the ground ice, and our last ebb or outgoing tide was hardly perceptible." 'The ice crust cannot sensibly affect (by friction on its lower surface) the progress of the tide wave, and will certainly not sensibly interfere (by friction on the ice foot and breakage of the ice fields) with the rise and fall of the tide.

Progress of the Tide Wre.-The tide at Van Rensselaer Harbor may be taken as a derived tide, and transmitted to it from the Atlantic Ocean, and in part modified by the small tide originating in the waters of Baffin's Bay; which latter tide, however, must necessarily be small, particularly on account of the general direction of the bay, which is rery unfavorable for the production of a ticle wave. That the tide wave is travelling up along the western coast of Greenland, or, in other words, reaches Van Rensselaer. Harbor from the sonthward, may be seen from the following observed establishments :-

Holsteinbory Harbor, latitule $66^{\circ} 56^{\prime}$, longitude $53^{\circ} 42^{\prime}$. ILigh water at F. \& C. $6^{\mathrm{h}} 30^{\mathrm{m}}$. Spring tides rise 10 fcet.-Capt. Inglefield, 185.3.

Whalefish Islamls (near 1)iseo), latitude $65^{\circ} 59^{\prime}$, longitude $53^{\circ} 133^{\prime}$. Time of

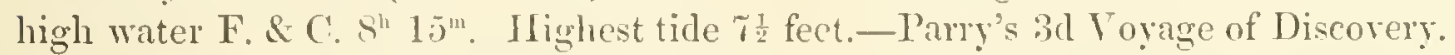

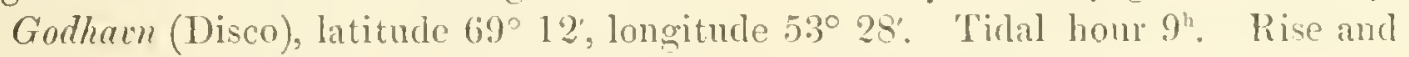
fall $7 \frac{1}{2}$ feet.-See Map in Narrative of Kiane's First Voyguge.

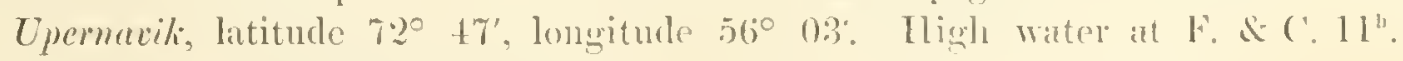
Rise 8 feet-Capt. Inglefield, 1854.

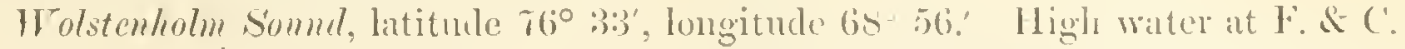
$11^{\mathrm{h}} \mathrm{S}^{\mathrm{m}}$. Rise, both at spring and neaps, $\mathrm{i}$ to $\mathrm{T}^{\frac{1}{2}}$ feet.--(See Admiralty Chart of Baffin's Bay, sheet 1, 185\%, corrected to 185\%.) The observations themselves, taken by Captain Sannders of H. M. S. North Star, in $18+9$ and 1850, were kindly fur- 
nisher to Prof. Bache by the Hydrographer to the Admiralty, Captrin J. Washington, R. N., and are given in the appendix to this paper. And finally,

Tan Rensseluer Harbor, latitude $75^{\circ} 3 \gamma^{\prime}$, longitude $70^{\circ} 53^{\prime}$. High water at F. \& $C .11^{\mathrm{b}} 50^{\mathrm{m}}$, as derived from the preceding analytical expression. Rise and fall at spring tide 11.1 feet, at neap tide 4.7 feet, arerage range 7.9 feet.

Br means of the difference in the establishments of Holsteinborg and Van Rensselacr, we can obtain an approximation to the depth of Baffin's Bay and Smith's Straits. viz:-

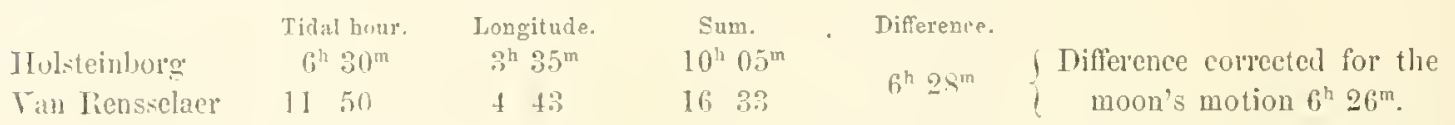

Assuming the distance along the channel to be 770 nautical miles, we have a relocity of the tide wave of about 202 fcet in a second, which, according to Airy's table (174), Tiles and Waves, wonld correspond to a depth of nearly 1300 feet, or about 2:20 fathoms-a result probably smaller than the true value, since the other observations indicate a greater depth, it may be taken as an inferior limit; in the same manner we find from the co tidal hours of Upernavik and Van Rensselaer a deptl of near s00 fathoms, and a similar result from the Wolstenholm observations; this last result may perhaps he taken as an upper limit.

Somdings.-The following soundings have been copied from the log-book:-

June 19, 18.is. Lat. $51^{\circ} 12^{\prime}, \operatorname{long} .520 \mathrm{~s}^{\prime}$ (goverument somuling twine snd 82-pound shot).

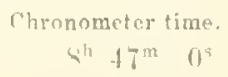

June 26, 1553 . Lat. $59^{\circ} 4 \mathrm{~s}^{\prime}$, long. $50^{\circ}: 3^{\prime}$ (goremment sounding twine and 32-pound shot).

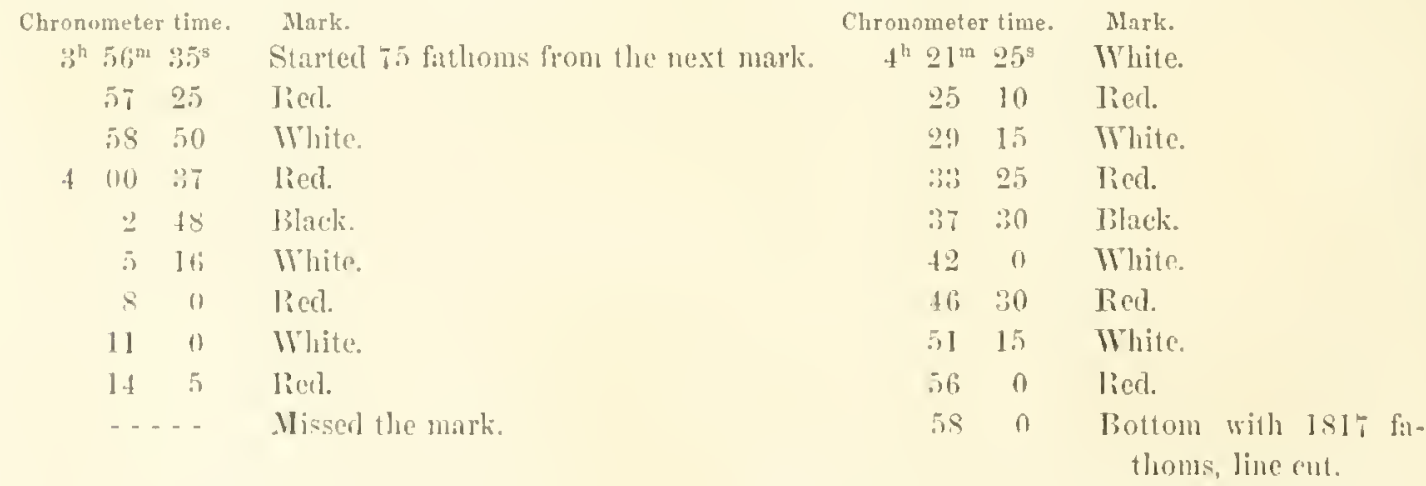

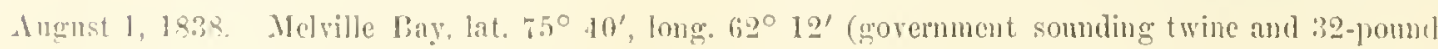
sliot).

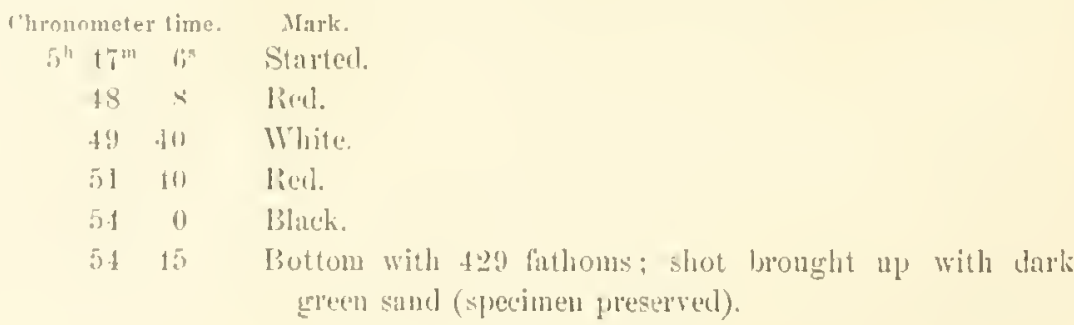




\section{A P P E N D I X.}

Tidat Oeshryations made on poari) II. M. S. Nurtil Star, Commander Saunders, at the Winter Quarters in Wolstenholi Sound. (Fron tile Simp's Log.)

\begin{tabular}{|c|c|c|c|c|c|c|c|c|c|}
\hline \multirow{2}{*}{$\begin{array}{c}\text { DAte. } \\
1849 .\end{array}$} & \multicolumn{2}{|c|}{ 1ligh water. } & \multicolumn{2}{|c|}{ Low water. } & \multirow{2}{*}{$\begin{array}{c}\text { DATE. } \\
1850 .\end{array}$} & \multicolumn{2}{|c|}{ High water. } & \multicolumn{2}{|c|}{ Low water. } \\
\hline & Time. & Ileight. & Time. & Height. & & Time. & Height. & Time. & Height \\
\hline Nov. $1 \mathrm{~d}, \mathrm{~A} . \mathrm{M}$. & $120 \mathrm{~m}$ & $\begin{array}{cc}\text { Ft. In. } \\
\text { is } & 0\end{array}$ & $4^{\text {nh }} 0^{112}$ & $\begin{array}{c}\text { Ft. In. } \\
694\end{array}$ & Mar. 13, P. Mr. & & Ft. In. & $6^{\text {h }} 0^{\mathrm{m}}$ & $\begin{array}{cc}\text { Ft. } & \text { In } \\
69 & 0\end{array}$ \\
\hline $\begin{array}{lll} & 17, \text { A. } 1 . \\
\text { " } 17, \text { P. } .1\end{array}$ & (1) 30 & $78 \quad 6$ & 530 & (1) 11 & " 19, A. 11 & $3^{\mathrm{h}} 0^{\mathrm{m}}$ & 75 & 110 & 7010 \\
\hline “ 29, A. M. & 110 & $7 ; ; 5$ & 50 & 714 & s6 $27, \mathrm{P}, \mathrm{Nl}$ & 11 & $76 \quad 2$ & 530 & (;) 1 \\
\hline$" 30$, A.M. & 100 & 791 & 430 & 7110 & April \&, A. MI. & & 10 & 120 & 699 \\
\hline Dec. 14, P. . I. & 110 & 696 & 0 & (i5) 2 & " $\quad 4,1$. M. & 40 & $7: 2$ & & \\
\hline "22, P. M. & 40 & 71) 9 & 10 30 & 654 & " 12, A. M. & 1130 & 701 & 5 & 69 \\
\hline " 20, A. . & 110 & 7310 & 330 & (j) 0 & “ 26, A. M. & 110 & 703 & 50 & 69 \\
\hline 1850. & & & & & Nay $4, \mathrm{l} . \mathrm{M}$. & 50 & 7.50 & & \\
\hline Jan. 13, A. 1. & 120 & 71 & 530 & $64 \quad 2$ & “ 19, A. M. & 530 & 772 & & \\
\hline " 21,1 . M. & 40 & 704 & 1030 & 651 & 19, P. M. & & & 1130 & 72 \\
\hline " 27, A. М. & 110 & 724 & 530 & $64 \quad 2$ & " $2 t, A . M$ & & 7.510 & $6 \quad 11$ & 69 \\
\hline Feb. 3, P. M. & 330 & 700 & 90 & 6310 & June 2, P. M. & 0 & is 11 & & \\
\hline " 19,1 . . .I. & 40 & 703 & 930 & 668 & " 23, P. 11. & 11 & $74 \quad 4$ & & \\
\hline March 5, P. M. & 430 & 500 & $10 n$ & 6010 & July $\quad$ Q, P. M. & 1111 & 7410 & 30 & $66 \quad 1$ \\
\hline " 13, A. M. & 1130 & 760 & & & & & & & \\
\hline
\end{tabular}

From the rongh manner with which the ahove observations appear to have been made, an approximate establisliment and rise of tide only ean be deduced from them.

II. W. F. and C. appears to take place between $\mathrm{XI}^{\mathrm{h}} 0^{\mathrm{m}}$ and $\mathrm{XI}^{\mathrm{h}} 15^{\mathrm{m}}$, say $\mathrm{XI}^{\mathrm{h}} \mathrm{S}^{\mathrm{m}}$, and the rise both at springs and neaps from 7 to $7 \frac{1}{2}$ feet.

(Signed)

JNO. BURWOOD, Mister R. N.,

(Tide Compulero).

Amiralty, $3 d . J u l y, 156 \%$. 



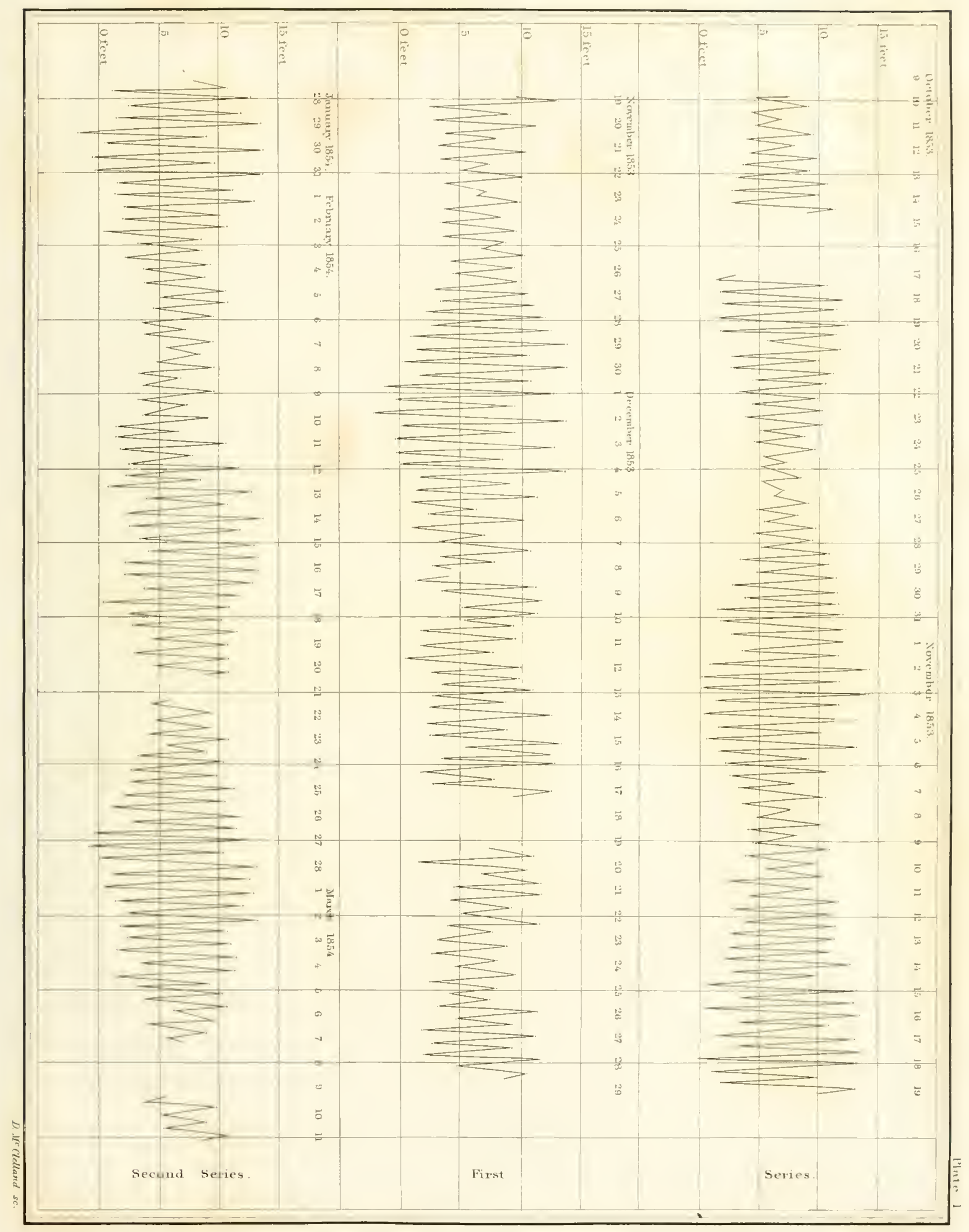





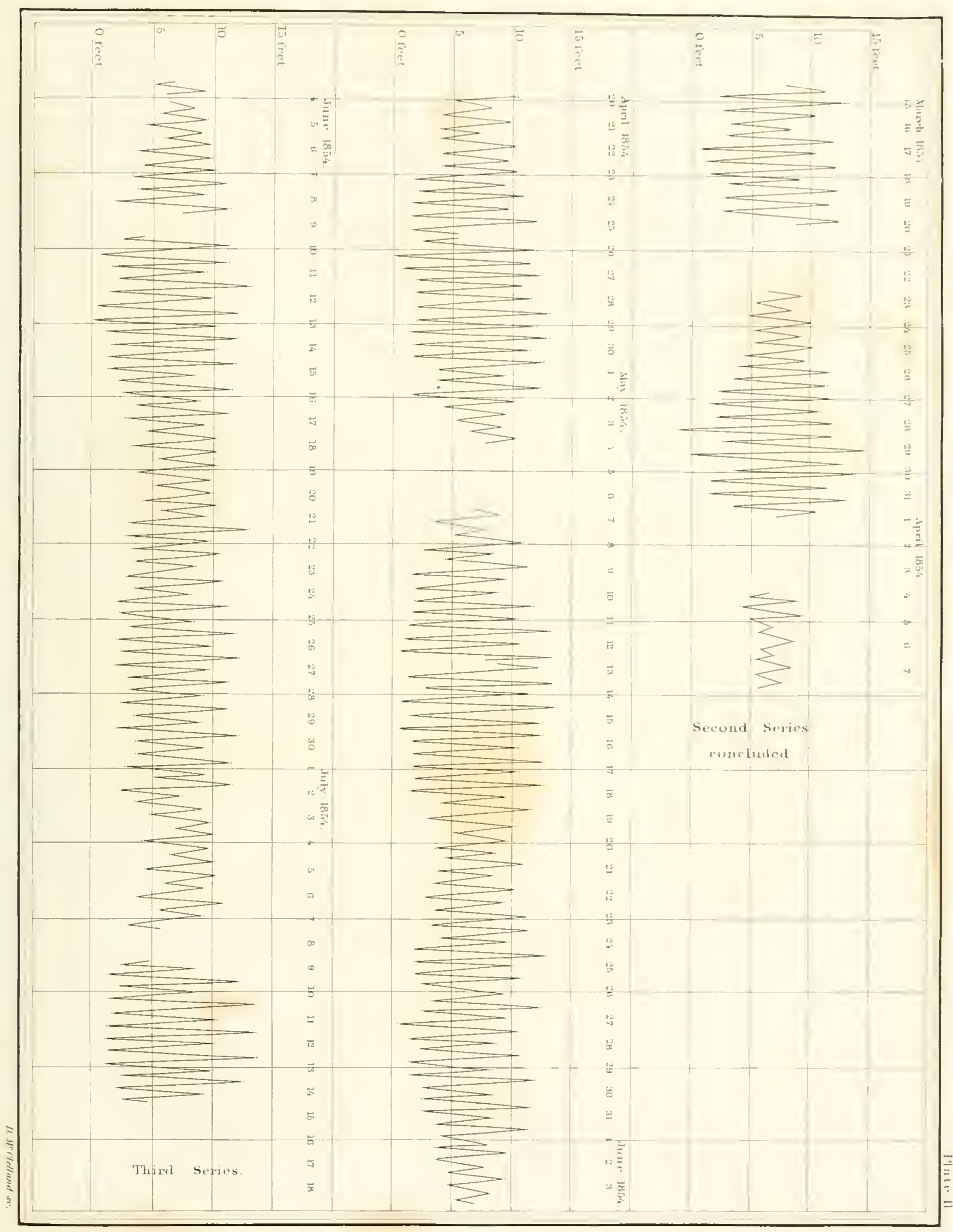





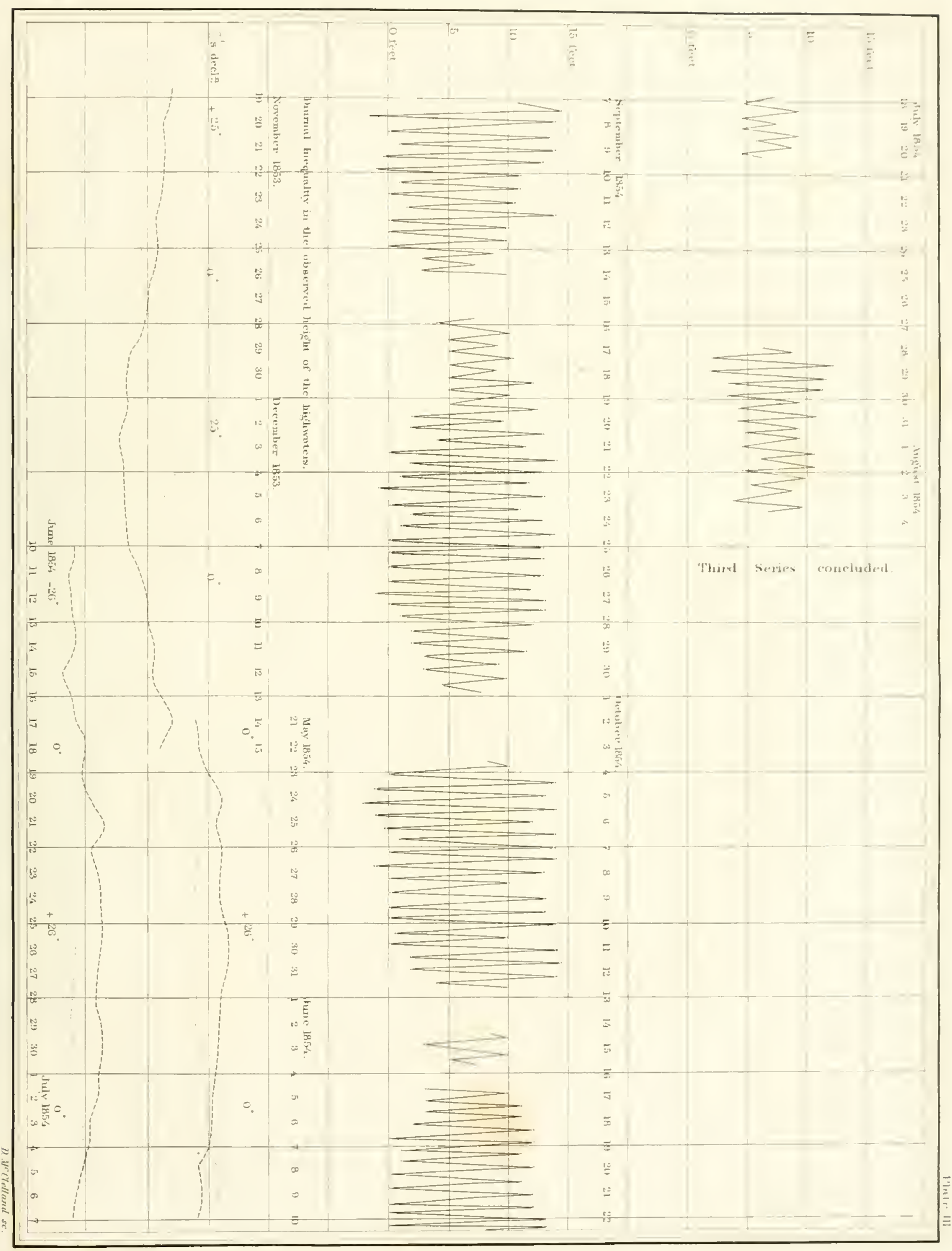





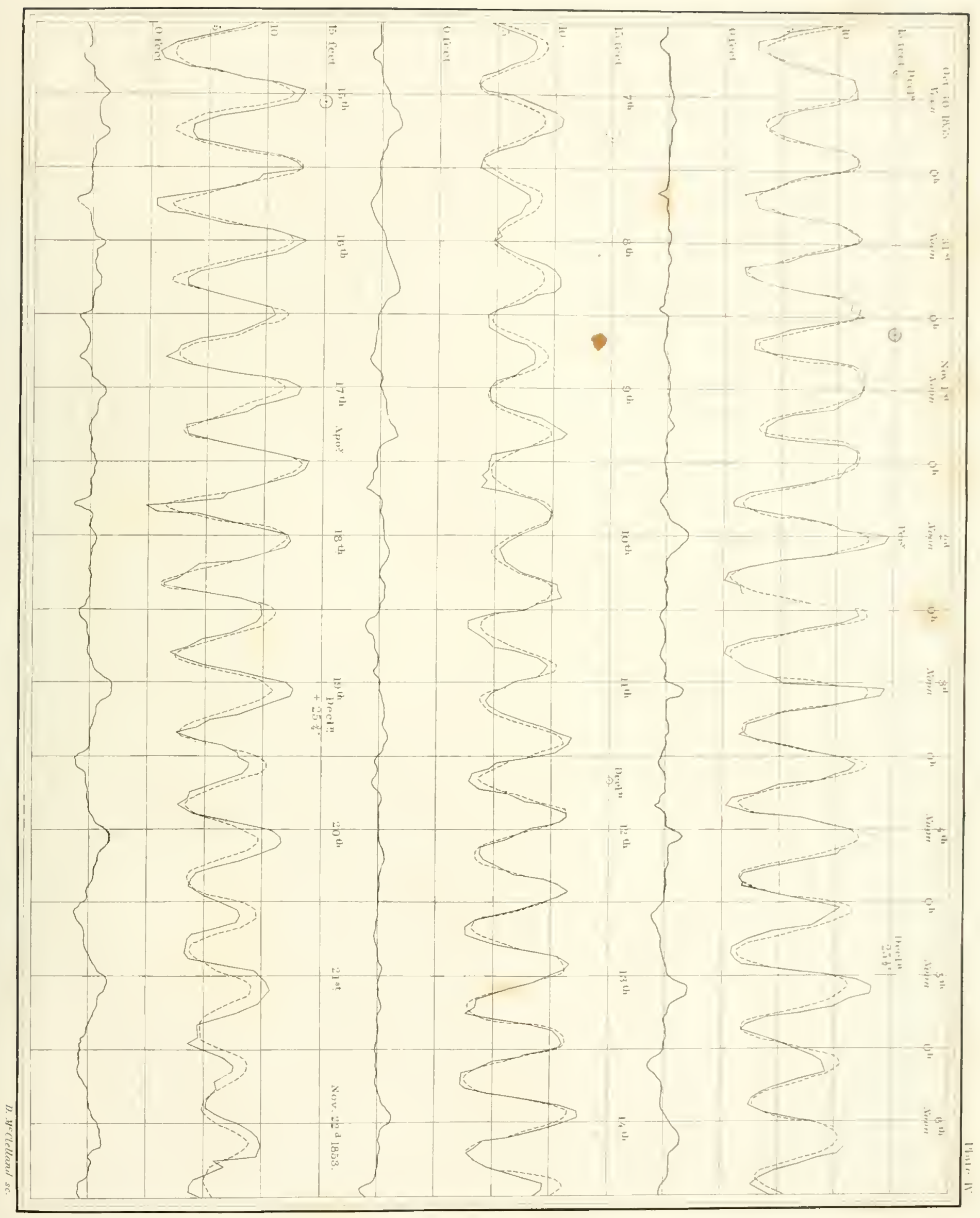






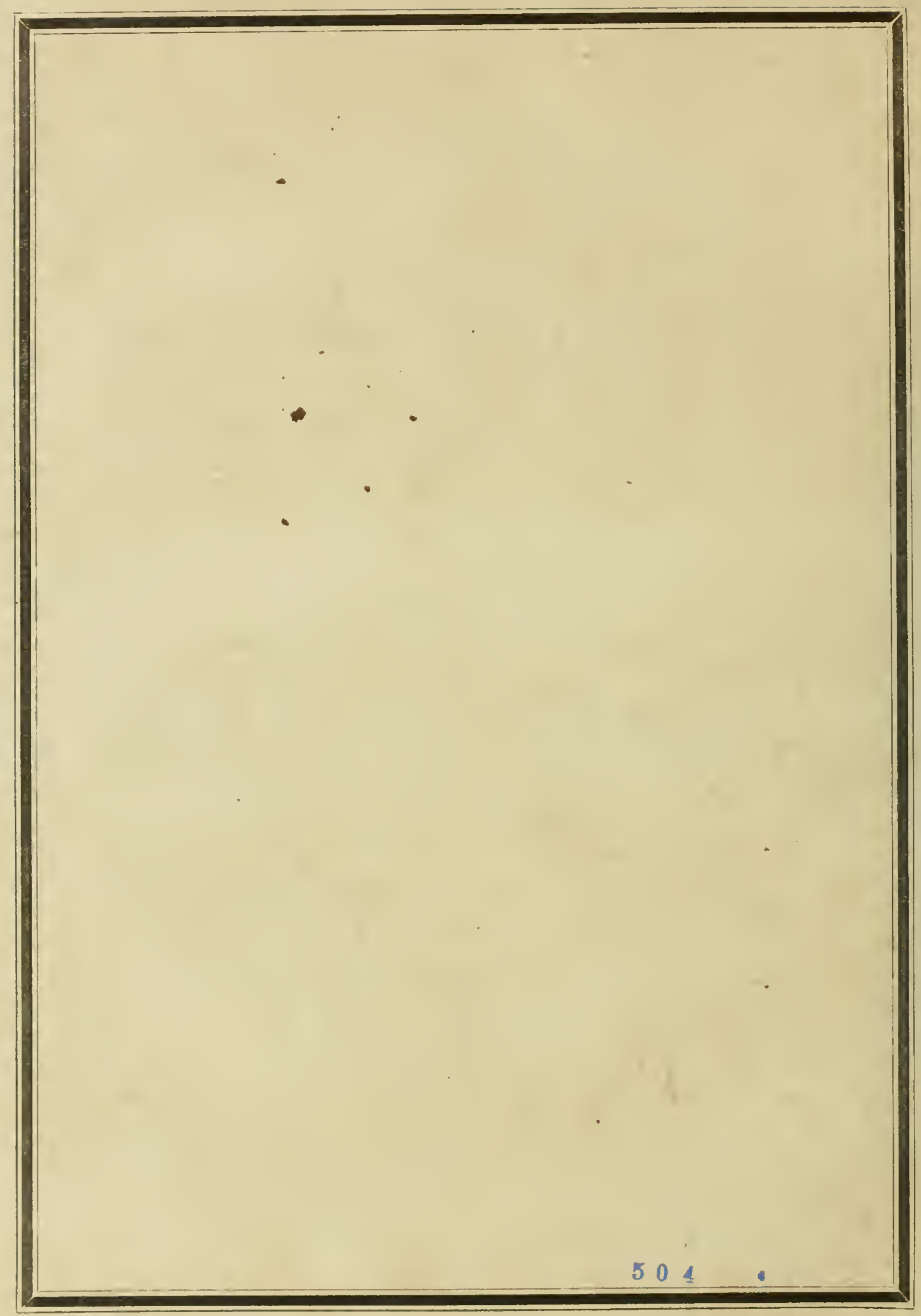





This book is DUE on the last date stamped below

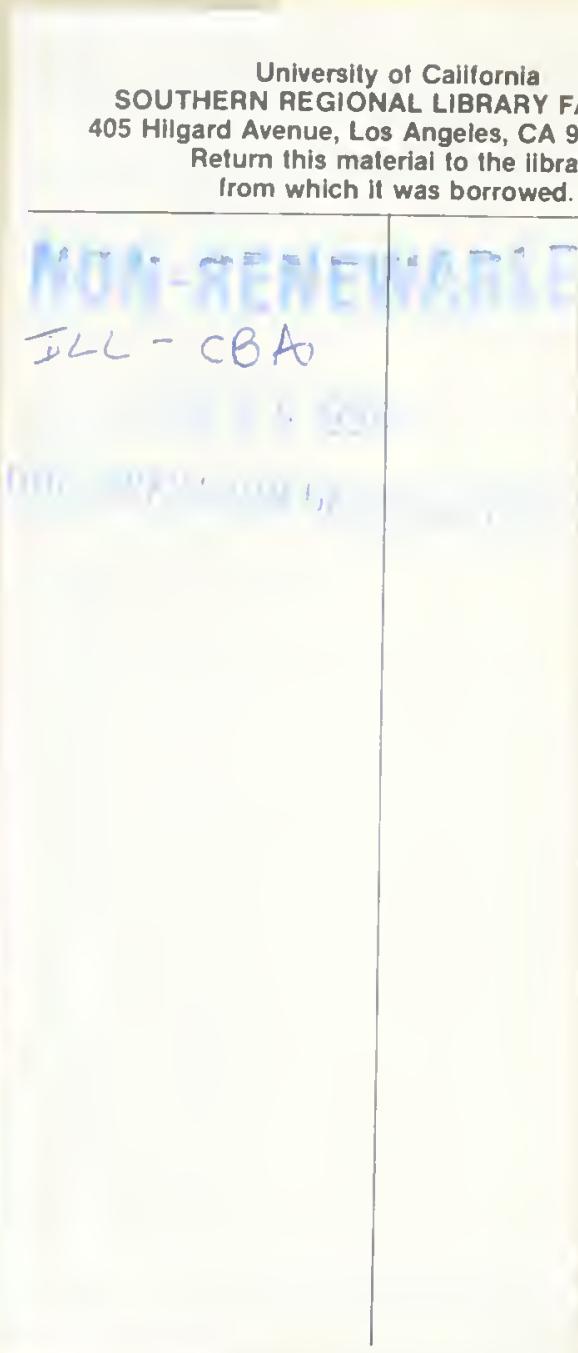


Kl3t Tidal obser-

vations in

the Arctic seas.

it 1958 Dariing

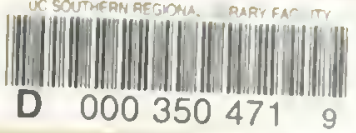

$\because V K$

607

Kl3t. 
